\title{
DR2011134 Long et al.
}

\section{Data Repository: Tectonostratigraphy of the Lesser Himalaya of Bhutan: Implications for the Along-Strike Stratigraphic Continuity of the Northern Indian Margin}

\section{Insert Figure DR1 here:}

Figure DR1: A) Thick-bedded, light-gray Shumar Formation quartzite in northern Kuru Chu valley, displaying tabular cross-bedding with planar foresets, showing upright orientation (15-cm pen for scale). B) Schist rich in quartz vein boudins, characteristic of Daling Formation (30-cm hammer for scale). C) Feldspar augen $\sigma$-clast in orthogneiss body in Daling Formation, showing top-to-the-south (south and north marked) sense of shear (15-cm pen for scale). D) Cliffs of white Baxa Group quartzite (road for scale). E) Photomicrograph (cross-polarized light) of quartz microstructure characteristic of Daling-Shumar Group quartzite, showing equant, polygonal texture characteristic of subgrain rotation recrystallization. F) Photomicrograph (cross-polarized light) of garnet-biotite-muscovite schist characteristic of Jaishidanda Formation. G) Photomicrograph (cross-polarized light) of quartzite characteristic of the Baxa Group, showing elongation of relict quartz grains, dynamic recrystallization by bulging at grain boundaries, and presence of potassium feldspar ( $\mathrm{kfsp})$. H) Lenticular bedding characteristic of Baxa Group quartzite (30 cm hammer for scale). I) Diuri Formation diamictite $(20 \mathrm{~cm}$ notebook for scale). J) Photomicrograph showing distinct bimodal grain size and deformation fabric characteristic of Diuri Formation diamictite. K) Dark-gray argillite outcrop near top of Gondwana succession section on road north of Samdrup Jongkhar.

\section{Discussion DR1: Methods of Arizona LaserChron Center U/Pb zircon dating}

Rock samples were crushed and pulverized to sand-size grains, separated by density on a Wilfley table, and then separated into dense and light fractions by a $3.32 \mathrm{~g} / \mathrm{cc}$ liquid separation. The dense fraction was passed through a Frantz magnetic separator, and the zircons were mounted in epoxy plugs and then polished to half-thickness. Photographic images were made of all zircon mounts to keep track of which grains were dated.

Material was ablated from the sample surface using a DUV193 Excimer laser system from New Wave Instruments. The laser operates at a wavelength of $193 \mathrm{~nm}$, and for the analyses in this study 35 micron-wide spot sizes were used, except for samples BU07-54, BU0755, and BU08-72, which contained smaller zircons and were hit with a 25 micron-diameter beam (see notes below Table DR1). For most analyses the laser was operated at minimum output energy $(\sim 40 \mathrm{~mJ})$ with a repetition rate of 8 pulses per second, which created a $\sim 15$ micron-deep pit for a typical 20 second analysis. The ablated material is carried in helium gas into the plasma source of a multicollector inductively coupled plasma mass spectrometer (an Isoprobe, from GV Instruments). This instrument is equipped with nine moveable Faraday collectors and four lowside Channeltrons (ion counters). Eight of the Faraday collectors use a $10^{11} \mathrm{ohm}$ resistor, whereas the Faraday used for measuring ${ }^{207} \mathrm{~Pb}$ is equipped with a $10^{12} \mathrm{ohm}$ resistor. This configuration allows static-mode measurement of all isotopes, using $10^{11}$ Faraday detectors for

${ }^{238} \mathrm{U},{ }^{232} \mathrm{Th},{ }^{208} \mathrm{~Pb}$, and ${ }^{206} \mathrm{~Pb}$, a $10{ }^{12}$ Faraday detector for ${ }^{207} \mathrm{~Pb}$, and an ion-counting channel for 
${ }^{204} \mathrm{~Pb}$. Each analysis consists of one 20-second integration on peaks with the laser off (for backgrounds), 20 or 12 one-second integrations with the laser firing, and a 30 second delay to purge the previous sample and prepare for the next analysis. Each analysis is evaluated for consistency of ${ }^{206} \mathrm{~Pb} /{ }^{238} \mathrm{U}$ and ${ }^{206} \mathrm{~Pb} /{ }^{207} \mathrm{~Pb}$ ratios through the 20 seconds of data acquisition. If ratios display either a sudden change, or a gradual increase greater than $\sim 5 \%$ for ${ }^{206} \mathrm{~Pb} /{ }^{238} \mathrm{U}$, the analysis is discarded. This ensures that analyses are not compromised by crossing an age boundary.

Common $\mathrm{Pb}$ correction was accomplished by using the measured ${ }^{204} \mathrm{~Pb}$ and assuming an initial $\mathrm{Pb}$ composition from Stacey and Kramers (1975). Conservative uncertainties of 1.0 for ${ }^{206} \mathrm{~Pb} /{ }^{204} \mathrm{~Pb}, 0.3$ for ${ }^{207} \mathrm{~Pb} /{ }^{204} \mathrm{~Pb}$, and 2.0 for ${ }^{208} \mathrm{~Pb} /{ }^{204} \mathrm{~Pb}$ were used for the composition of the common $\mathrm{Pb} .{ }^{204} \mathrm{Hg}$ present in the argon plasma gas, as well as any background ${ }^{204} \mathrm{~Pb}$ or molecular 204, was accounted for by first measuring backgrounds in the 204 mass position, then measuring the peak 204 intensity with the laser firing, and subtracting the background intensity from the peak intensity.

Fractionation of $\mathrm{Pb} / \mathrm{U}$ and $\mathrm{Pb} / \mathrm{Th}$ occurs primarily in the laser pit, and is highly sensitive to the rate of carrier gas flow across the sample surface. An optimal balance between signal intensity and stability occurs at a carrier gas flow rate of $0.45 \mathrm{ml} / \mathrm{minute}$, which generates a $\mathrm{Pb} / \mathrm{U}$ sensitivity of 0.9 (e.g., a $500 \mathrm{Ma}$ zircon yields a ${ }^{206} \mathrm{~Pb} /{ }^{238} \mathrm{U}$ age of $450 \mathrm{Ma}$ ). To correct for $\mathrm{Pb} / \mathrm{U}$ and $\mathrm{Th} / \mathrm{U}$ fractionation, standards were analyzed once every 5 unknowns. Fractionation standards for zircon are fragments of a large Sri Lanka zircon crystal that yields an age of 563.5 $\pm 3.2 \mathrm{Ma}$ (2-sigma, ID-TIMS) (Gehrels et al., 2008). The unknowns are corrected for the closest 6 standards using a sliding window average. The error on this fractionation factor is generally $\sim 1 \%$ (2-sigma) for ${ }^{206} \mathrm{~Pb} /{ }^{238} \mathrm{U}$ ages. Fractionation of $\mathrm{Pb}$ isotopes is minimal, with a maximum of $\sim 3 \%$ fractionation of ${ }^{206} \mathrm{~Pb} /{ }^{207} \mathrm{~Pb}$. This fractionation is also removed by comparison with standards, using the same procedure described above. The error on this fractionation factor is generally $\sim 1 \%$ (2-sigma) for ${ }^{206} \mathrm{~Pb} /{ }^{207} \mathrm{~Pb}$ ages. $\mathrm{Pb} / \mathrm{U}$ and $\mathrm{Pb} / \mathrm{Th}$ fractionation varies with depth during laser ablation, increasing by $\sim 5 \%$ during a 20 -second analysis that excavates to a depth of 15 microns. This was accounted for by monitoring the depth-related fractionation of standards, and then applying a sliding-window depth-related fractionation factor to the unknowns. $\mathrm{Pb} / \mathrm{U}$ fractionation also varies by up to several percent depending on position on the mount surface, due to variations in the flow rate/pattern of the helium carrier gas across the sample surface. For this reason, all standards and unknowns are mounted close together in the central portion of the mount, and care was taken to analyze standards that are as close as possible to each unknown.

To determine accurate concentrations of $U$ and $T h$, we compare intensities with the Sri Lanka standard, which has concentrations of $\mathrm{U}, \mathrm{Th}$, and $\mathrm{Pb}$ known to $\sim 20 \%$. For each zircon analysis, the errors in determination of ${ }^{206} \mathrm{~Pb} /{ }^{238} \mathrm{U}$ and ${ }^{206} \mathrm{~Pb} /{ }^{204} \mathrm{~Pb}$ result in a measurement error of $\sim 1-2 \%$ (at 2-sigma level) in the ${ }^{206} \mathrm{~Pb} /{ }^{238} \mathrm{U}$ age. The errors in measurement of ${ }^{206} \mathrm{~Pb} /{ }^{207} \mathrm{~Pb}$ and ${ }^{206} \mathrm{~Pb} /{ }^{204} \mathrm{~Pb}$ also result in $\sim 1-2 \%$ (at 2-sigma level) uncertainty in age for grains that are $>1.0 \mathrm{Ga}$, but are substantially larger for younger grains due to low intensity of the ${ }^{207} \mathrm{~Pb}$ signal. We refer to errors that arise from the measurement of ${ }^{206} \mathrm{~Pb} /{ }^{238} \mathrm{U},{ }^{206} \mathrm{~Pb} /{ }^{207} \mathrm{~Pb}$, and ${ }^{206} \mathrm{~Pb} /{ }^{204} \mathrm{~Pb}$ as random (or measurement) errors, because they are different for each analysis within a session. For most analyses, the cross-over in precision of these random errors for ${ }^{206} \mathrm{~Pb} /{ }^{238} \mathrm{U}$ and ${ }^{206} \mathrm{~Pb} /{ }^{207} \mathrm{~Pb}$ ages occurs at $\sim 1.0 \mathrm{Ga}$. For this reason, ${ }^{206} \mathrm{~Pb} /{ }^{238} \mathrm{U}$ ratios were considered the most representative, and were used for analyses younger than $\sim 1.0 \mathrm{Ga}$, and ${ }^{207} \mathrm{~Pb} * /{ }^{206} \mathrm{~Pb}^{*}$ ratios were considered the most representative, and were used for ages older than $\sim 1.0 \mathrm{Ga}$. Table DR2 shows the cutoff ages used for individual samples; these cutoff ages were chosen as close to $\sim 1.0 \mathrm{Ga}$ as possible 
without dividing an age peak artificially (for example, for an age cluster between 900-1050 Ma, cutoff would be at $1060 \mathrm{Ma}$, or for a cluster between 1000-1200 Ma, cutoff would be at $980 \mathrm{Ma}$ ).

Data table DR1 reports analytical data at 1-sigma uncertainties based on the analytical (or measurement) errors. For determination of the crystallization age of sample NBH-8 and the determination of the maximum deposition ages of samples BU07-10 and NBH-9 (see sections 5.2. and 6.1. in text for further discussion), the final age was determined from the weighted mean from Ludwig (2003) of the 3 youngest concordant and overlapping (within error) analyses. The uncertainty of the weighted mean is based on the scatter and precision of the set of concordant ages, weighted according to their measurement errors. The systematic errors are then added to this measurement error quadratically. Systematic errors include contributions from the fractionation correction, composition of common $\mathrm{Pb}$, age of the calibration standard, and $\mathrm{U}$ decay constants. Total average systematic errors are listed for individual samples in Table DR2.

For detrital zircon samples, approximately 100 randomly-selected zircon crystals were analyzed from each sample, to identify each of the main age groups present. Data were filtered according to precision (typically 10\% cutoff) and discordance (typically $30 \%$ cutoff) and plotted on $\mathrm{Pb} / \mathrm{U}$ concordia diagrams or relative age-probability plots using algorithms of Ludwig (2003) (Fig. DR2). Relative age probability curves (Fig. 5) were constructed by: (1) calculating a normal distribution for each analysis based on the reported age and uncertainty, (2) summing the probability distributions of all acceptable analyses into a single curve, and (3) if normalized, dividing the area under the curve by the number of analyses.

Interpretations of the significance of $\mathrm{U} / \mathrm{Pb}$ detrital zircon data, and the justification of the $30 \%$ accepted range of discordance, are based on the view that only clusters of ages record robust sources ages. This is because a single age determination may be compromised by $\mathrm{Pb}$ loss or inheritance (even if concordant), whereas it is unlikely that two or more grains that have experienced $\mathrm{Pb}$ loss or inheritance would yield the same age. We accordingly attach age significance only to clusters defined by three or more overlapping (within error) analyses; this has particular importance for determination of the youngest age component in a detrital zircon sample, which is commonly used as a maximum depositional age (see caption for Figure DR3 for discussion of youngest peak for samples in this study).

For further discussion of the analytical methods of the University of Arizona LaserChron Center, refer to Gehrels et al. (2006; 2008).

\section{Insert Table DR1 here:}

Table DR1: U-Pb (zircon) geochronologic analyses by Laser-Ablation Multicollector ICP Mass Spectrometry

\section{Insert Table DR2 here:}

Table DR2: Total average systematic errors (s.e.) for ${ }^{206} \mathrm{~Pb} /{ }^{238} \mathrm{U}$ and ${ }^{206} \mathrm{~Pb} /{ }^{207} \mathrm{~Pb}$ for each sample $(2 \sigma)$, and average ${ }^{206} \mathrm{~Pb} /{ }^{238} \mathrm{U}$ ages and standard deviation calculated for all standards run for each sample ( $\mathrm{n}=24$ for a typical 100 -analysis detrital zircon sample). Systematic errors not available for sample BU08-72 due to corrupt file. Also shown are cutoff ages for each sample; ${ }^{206} \mathrm{~Pb} /{ }^{207} \mathrm{~Pb}$ ages were considered the best age for grains older than the cutoff age, and ${ }^{206} \mathrm{~Pb} /{ }^{238} \mathrm{U}$ ages were 
considered the best age for grains younger than the cutoff age (listed in Table DR1). Cutoff ages were chosen as close to $\sim 1.0 \mathrm{Ga}$ as possible without dividing an age peak (see Discussion DR1 above).

\section{Insert Figure DR2 here:}

Figure DR2: $\mathrm{Pb} / \mathrm{U}$ concordia plots of Bhutan LH samples. Includes all analyses greater with concordance $>70 \%$. Data for individual zircon analyses listed in Table DR1. Error ellipses are shown at the $1 \sigma$ level $(68.3 \%$ confidence $)$.

\section{Insert Figure DR3 here:}

Figure DR3: $\mathrm{Pb} / \mathrm{U}$ concordia plots of Jaishidanda Formation (BU08-72, BU08-135) and Baxa Group (BU08-22) samples, scaled for evaluation of robustness of youngest detrital zircon peaks. Includes all analyses with concordance $>70 \%$. Data for individual zircon analyses listed in Table DR1. Error ellipses are shown at the $1 \sigma$ level (68.3\% confidence). Note that sample BU08-72 has two concordant grains with $\sim 520$ and $\sim 549 \mathrm{Ma}{ }^{206} \mathrm{~Pb} /{ }^{238} \mathrm{~Pb}$ ages (grains 21, 4; Table DR1). Note that while this does not qualify as a significant (supported by 3 or more grains) peak under our definition, only 12 grains were analyzed for this sample. Note that the two youngest grains (ellipses marked in blue) in sample BU08-135 are 399 Ma (grain 101; Table DR1), which does not overlap the concordia line, and $421 \mathrm{Ma}$ (grain 66; Table DR1), which does. However, these young analyses do not define a significant peak; the youngest significant peak defined by 3 grains that overlap the concordia line (grains 70, 13, 92; Table DR 1 ) is centered at $\sim 475$ Ma. Data for grain 101 was not included in Fig. 5. Note that sample BU07-22 has 5 grains (ellipses marked in blue) with ${ }^{206} \mathrm{~Pb} /{ }^{238} \mathrm{U}$ ages between 461 and $500 \mathrm{Ma}$ (grains 73, 75, $84,82,81$; Table DR 1). However, note that only one of these grains (grain 82, 97.6\% concordant) overlaps the concordia line; grains 73 and 81 (77.8\%,87.0\% concordant) are completely off, and grains 75 and $84(81.7 \%, 81.4 \%$ concordant) touch the concordia line only at the very edge of the $1 \sigma$ error ellipse. The reported ${ }^{206} \mathrm{~Pb} /{ }^{238} \mathrm{U}$ ages of these grains that do not overlap the concordia line could be the result of $\mathrm{Pb}$-loss or mixing. Because of a lack $\mathrm{CL}$ imaging of analyzed zircons for determining whether or not laser spots overlapped a core and an igneous or metamorphic overgrowth, we cannot distinguish between these two options with grains that are this discordant. For this reason, we do not interpret the $\sim 490$ peak defined by grains $75,84,82$ as significant, because only grain 82 overlaps the concordia line. We interpret the peak supported by 6 concordant grains $(32,23,15,10,99$, and 66 ; all $>91 \%$ concordant; Table DR1), and centered at $\sim 525 \mathrm{Ma}$ (from AGEPICK program available at Arizona Laserchron Center website) as the youngest significant and robust detrital zircon age peak in this sample. Data for the young grains that don't fall on the concordia line $(73,75,84$, and 81) were not included in Fig. 5.

\section{Insert Table DR3 here:}

Table DR3: $\delta 13 \mathrm{C}$ and $\delta 18 \mathrm{O}$ values for Baxa Group dolomite (duplicates in bold) 


\section{References Cited}

Gehrels, G., Valencia, V., and Pullen, A., 2006, Detrital Zircon Geochronology by Laser Ablation Multicollector ICPMS at the Arizona LaserChron Center, in Olszewski, T., ed., Geochronology: Emerging Opportunities: Paleontology Society Papers, v. 12, p. 67-76.

Gehrels, G.E., Valencia, V., and Ruiz, J., 2008, Enhanced precision, accuracy, efficiency, and spatial resolution of $\mathrm{U}-\mathrm{Pb}$ ages by laser ablation multicollector inductively coupled plasma mass spectrometry: Geochemistry, Geophysics, Geosystems, v. 9, Q03017, doi:10.1029/2007GC001805

Ludwig, K.J., 2003, Isoplot 3.00: Berkeley Geochronology Center Special Publication 4, 70 p.

Stacey, J.S., and Kramers, J.D., 1975, Approximation of terrestrial lead isotope evolution by a two-stage model: Earth and Planetary Science Letters, v. 26, p. 207-221. 

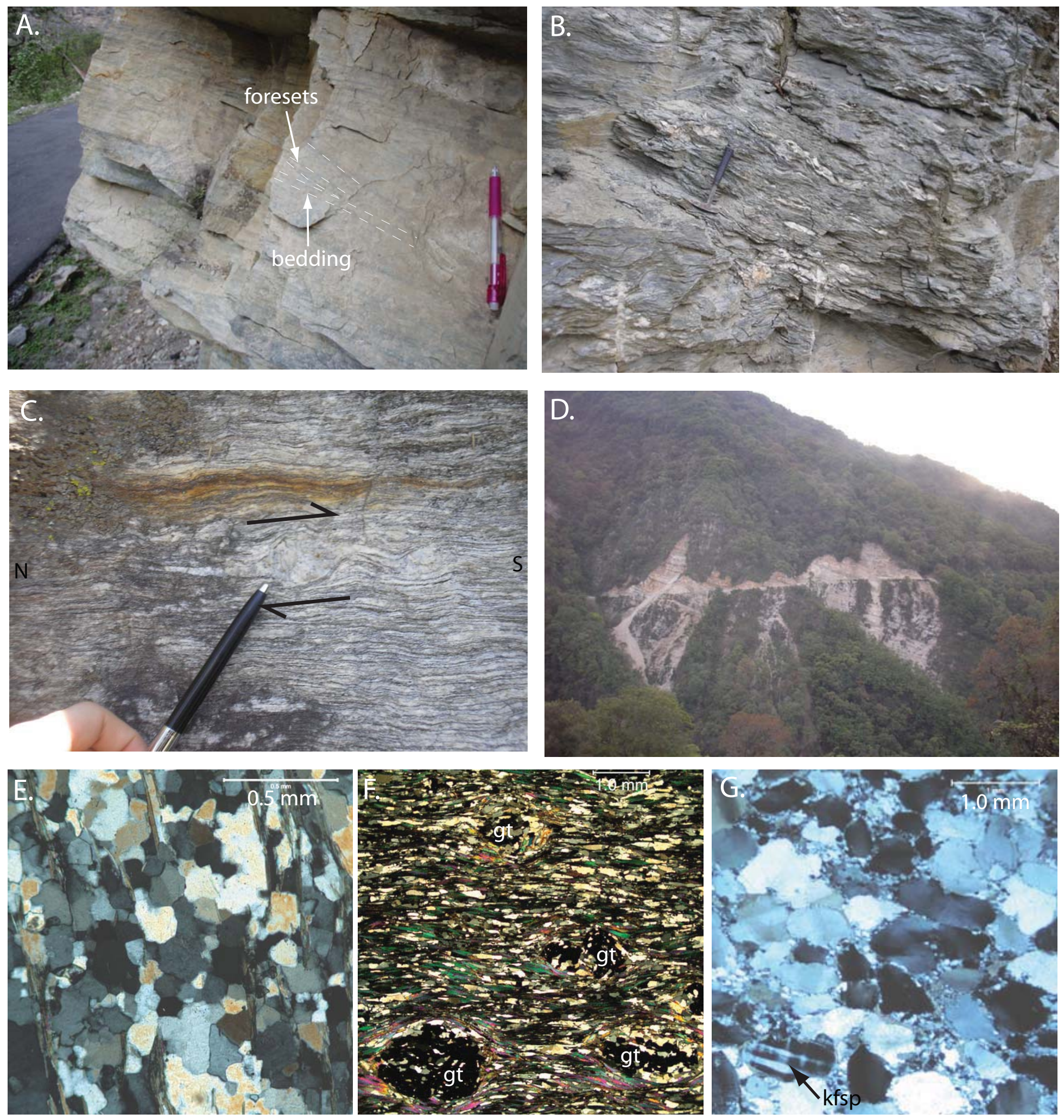

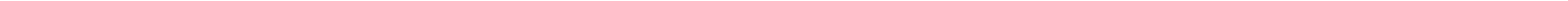

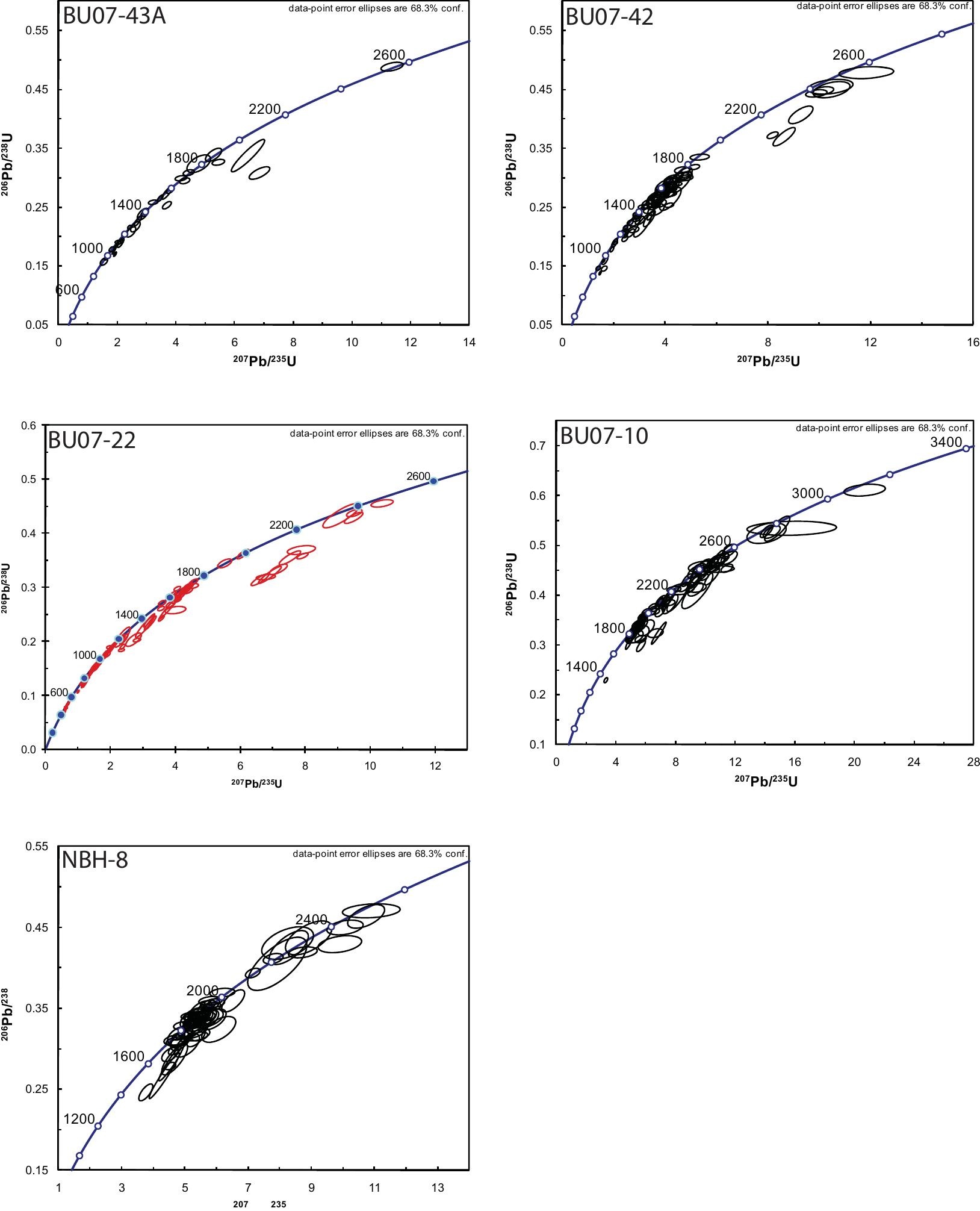

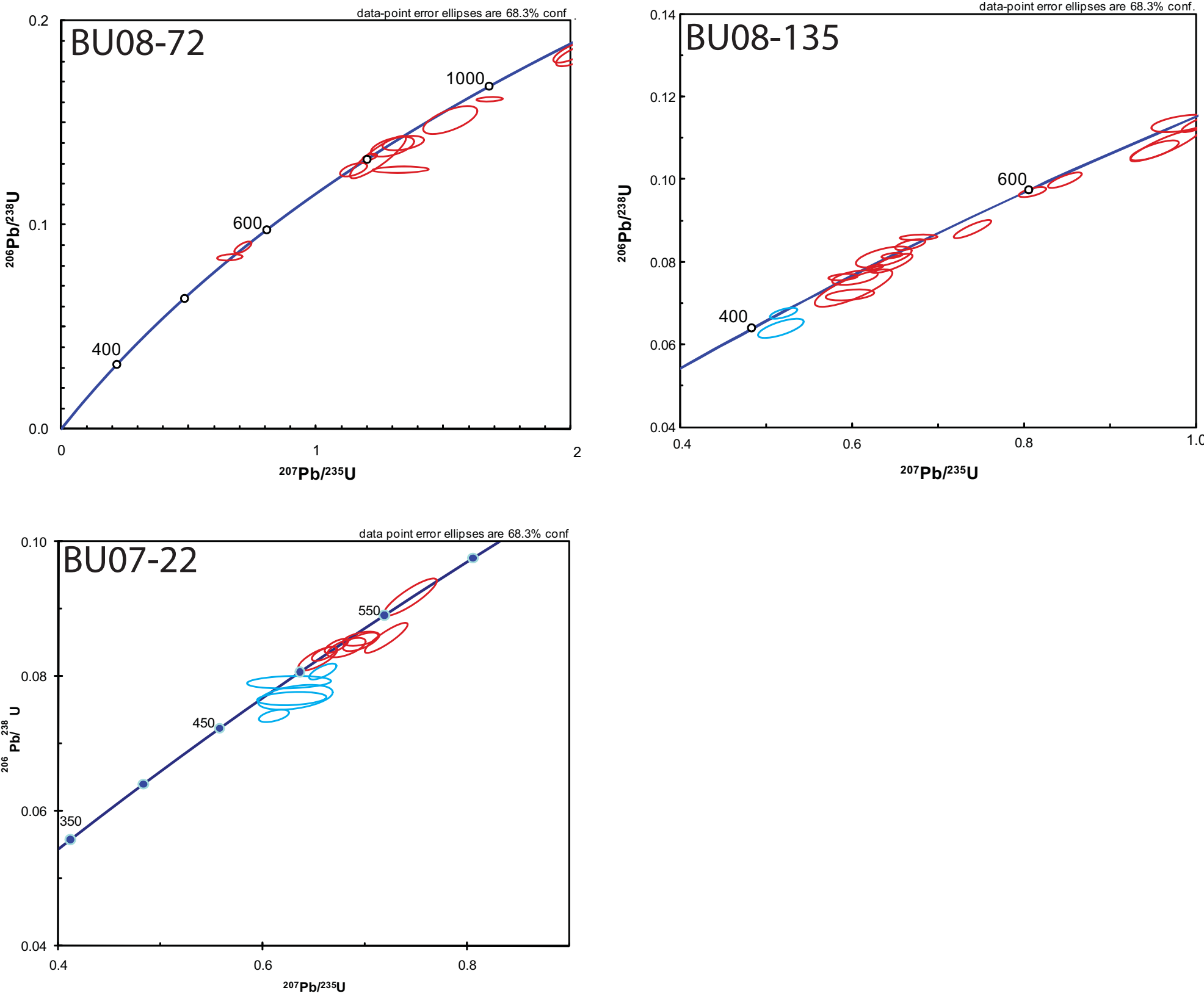


\begin{tabular}{|c|c|c|c|c|c|c|c|c|c|c|c|c|c|c|c|c|c|c|c|}
\hline & & & & & & & $\begin{array}{l}\text { Isotop } \\
\text { e } \\
\text { ratios }\end{array}$ & & & & & & $\begin{array}{l}\text { Apparent ages } \\
\text { (Ma) }\end{array}$ & & & & & & \\
\hline & & & & & & & & & & & & & & & & & & & \\
\hline Analysis & $\mathrm{U}$ & $206 \mathrm{~Pb}$ & $\begin{array}{c}\mathrm{U} / \mathrm{T} \\
\mathrm{h}\end{array}$ & $\begin{array}{c}206 \mathrm{~Pb} \\
*\end{array}$ & \pm & 207Pb* & \pm & $\begin{array}{c}206 P \\
b^{*}\end{array}$ & \pm & $\begin{array}{l}\text { err } \\
\text { or }\end{array}$ & $\begin{array}{c}206 P \\
b^{*}\end{array}$ & \pm & $207 \mathrm{~Pb}^{*}$ & \pm & $\begin{array}{c}206 \mathrm{P} \\
b^{*}\end{array}$ & \pm & $\begin{array}{l}\text { Best } \\
\text { age }\end{array}$ & \pm & $\begin{array}{l}\text { Con } \\
\text { c. }\end{array}$ \\
\hline & $\begin{array}{l}(\mathrm{pp} \\
\mathrm{m})\end{array}$ & $204 \mathrm{~Pb}$ & & $\begin{array}{c}207 \mathrm{~Pb} \\
*\end{array}$ & (\%) & $235 U^{*}$ & $(\%)$ & $238 U$ & $\begin{array}{c}(\% \\
)\end{array}$ & $\begin{array}{c}\text { cor } \\
\text { r. }\end{array}$ & $\begin{array}{c}238 U \\
*\end{array}$ & $\begin{array}{c}\text { (Ma } \\
\text { ) }\end{array}$ & $235 \mathrm{U}$ & $\begin{array}{c}\text { (Ma } \\
\text { ) }\end{array}$ & $\begin{array}{c}207 \mathrm{P} \\
b^{*}\end{array}$ & $\begin{array}{c}\text { (Ma } \\
\text { ) }\end{array}$ & (Ma) & $\begin{array}{c}\text { (Ma } \\
\text { ) }\end{array}$ & $(\%)$ \\
\hline $\begin{array}{l}\text { BU0710- } \\
99\end{array}$ & $\begin{array}{r}317 \\
5 \\
\end{array}$ & 35915 & 2.1 & $\begin{array}{r}9.510 \\
9 \\
\end{array}$ & 2.1 & 3.3178 & 2.5 & $\begin{array}{r}0.228 \\
9 \\
\end{array}$ & $\begin{array}{r}1 . \\
4\end{array}$ & $\begin{array}{r}0.5 \\
7 \\
\end{array}$ & $\begin{array}{r}1328 . \\
5 \\
\end{array}$ & 17.3 & 1485.2 & 19.5 & $\begin{array}{r}1716 . \\
8 \\
\end{array}$ & 37.7 & 1716.8 & 37.7 & 77.4 \\
\hline $\begin{array}{l}\text { BU0710- } \\
73\end{array}$ & 524 & $\begin{array}{r}16442 \\
0 \\
\end{array}$ & 2.5 & $\begin{array}{r}9.066 \\
1 \\
\end{array}$ & 1.2 & 4.8031 & 2.1 & $\begin{array}{r}0.315 \\
8 \\
\end{array}$ & $\begin{array}{l}1 . \\
7 \\
\end{array}$ & $\begin{array}{r}0.8 \\
2\end{array}$ & $\begin{array}{r}1769 . \\
3\end{array}$ & 26.3 & 1785.4 & 17.5 & $\begin{array}{r}1804 . \\
4\end{array}$ & 21.8 & 1804.4 & 21.8 & 98.1 \\
\hline $\begin{array}{l}\text { BU0710- } \\
61\end{array}$ & $\begin{array}{r}128 \\
4\end{array}$ & $\begin{array}{r}38057 \\
0 \\
\end{array}$ & 2.2 & $\begin{array}{r}8.797 \\
6 \\
\end{array}$ & 2.0 & 5.1976 & 2.7 & $\begin{array}{r}0.331 \\
6 \\
\end{array}$ & $\begin{array}{r}1 . \\
8 \\
\end{array}$ & $\begin{array}{r}0.6 \\
7 \\
\end{array}$ & $\begin{array}{r}1846 . \\
3 \\
\end{array}$ & 29.4 & 1852.2 & 23.2 & $\begin{array}{r}1858 . \\
8 \\
\end{array}$ & 36.5 & 1858.8 & 36.5 & 99.3 \\
\hline $\begin{array}{l}\text { BU0710- } \\
79\end{array}$ & 557 & $\begin{array}{r}20027 \\
5 \\
\end{array}$ & 3.6 & $\begin{array}{r}8.787 \\
6 \\
\end{array}$ & 2.8 & 5.0891 & 4.9 & $\begin{array}{r}0.324 \\
3 \\
\end{array}$ & $\begin{array}{r}4 . \\
0\end{array}$ & $\begin{array}{r}0.8 \\
1\end{array}$ & $\begin{array}{r}1810 . \\
9\end{array}$ & 62.7 & 1834.3 & 41.4 & $\begin{array}{r}1860 . \\
9\end{array}$ & 51.1 & 1860.9 & 51.1 & 97.3 \\
\hline BU0710-7 & 383 & $\begin{array}{r}13049 \\
5 \\
\end{array}$ & 3.5 & $\begin{array}{r}8.783 \\
7 \\
\end{array}$ & 2.6 & 5.3260 & 2.8 & $\begin{array}{r}0.339 \\
3 \\
\end{array}$ & $\begin{array}{r}1 . \\
2 \\
\end{array}$ & $\begin{array}{r}0.4 \\
3 \\
\end{array}$ & $\begin{array}{r}1883 . \\
3 \\
\end{array}$ & 19.9 & 1873.0 & 24.3 & $\begin{array}{r}1861 . \\
7 \\
\end{array}$ & 46.4 & 1861.7 & 46.4 & $\begin{array}{r}101 . \\
2\end{array}$ \\
\hline $\begin{array}{l}\text { BU0710- } \\
80\end{array}$ & 969 & $\begin{array}{r}31158 \\
5 \\
\end{array}$ & 8.6 & $\begin{array}{r}8.737 \\
0 \\
\end{array}$ & 1.7 & 5.3101 & 2.0 & $\begin{array}{r}0.336 \\
5 \\
\end{array}$ & $\begin{array}{r}1 . \\
2 \\
\end{array}$ & $\begin{array}{r}0.5 \\
8 \\
\end{array}$ & $\begin{array}{r}1869 . \\
7\end{array}$ & 19.0 & 1870.5 & 17.3 & $\begin{array}{r}1871 . \\
3 \\
\end{array}$ & 29.8 & 1871.3 & 29.8 & 99.9 \\
\hline $\begin{array}{l}\text { BU0710- } \\
17\end{array}$ & 392 & 83195 & 4.0 & $\begin{array}{r}8.654 \\
4 \\
\end{array}$ & 2.9 & 5.1996 & 3.2 & $\begin{array}{r}0.326 \\
4 \\
\end{array}$ & $\begin{array}{r}1 . \\
4 \\
\end{array}$ & $\begin{array}{r}0.4 \\
2 \\
\end{array}$ & $\begin{array}{r}1820 . \\
8 \\
\end{array}$ & 21.7 & 1852.6 & 27.6 & $\begin{array}{r}1888 . \\
4 \\
\end{array}$ & 52.9 & 1888.4 & 52.9 & 96.4 \\
\hline $\begin{array}{l}\text { BU0710- } \\
34\end{array}$ & 327 & $\begin{array}{r}12451 \\
5 \\
\end{array}$ & 2.3 & $\begin{array}{r}8.653 \\
6 \\
\end{array}$ & 2.0 & 4.9854 & 2.9 & $\begin{array}{r}0.312 \\
9 \\
\end{array}$ & $\begin{array}{r}2 . \\
1\end{array}$ & $\begin{array}{r}0.7 \\
2\end{array}$ & $\begin{array}{r}1754 . \\
9\end{array}$ & 32.0 & 1816.8 & 24.3 & $\begin{array}{r}1888 . \\
6 \\
\end{array}$ & 35.6 & 1888.6 & 35.6 & 92.9 \\
\hline $\begin{array}{l}\text { BU0710- } \\
13\end{array}$ & 394 & $\begin{array}{r}18202 \\
5 \\
\end{array}$ & 2.1 & $\begin{array}{r}8.639 \\
4 \\
\end{array}$ & 1.7 & 5.4703 & 2.0 & $\begin{array}{r}0.342 \\
8 \\
\end{array}$ & $\begin{array}{r}1 . \\
2 \\
\end{array}$ & $\begin{array}{r}0.5 \\
9 \\
\end{array}$ & $\begin{array}{r}1899 . \\
9\end{array}$ & 19.9 & 1895.9 & 17.6 & $\begin{array}{r}1891 . \\
6\end{array}$ & 29.7 & 1891.6 & 29.7 & $\begin{array}{r}100 . \\
4\end{array}$ \\
\hline $\begin{array}{l}\text { BU0710- } \\
60\end{array}$ & 869 & $\begin{array}{r}13306 \\
0 \\
\end{array}$ & 2.6 & $\begin{array}{r}8.588 \\
0 \\
\end{array}$ & 1.5 & 5.3701 & 2.8 & $\begin{array}{r}0.334 \\
5 \\
\end{array}$ & $\begin{array}{r}2 . \\
4 \\
\end{array}$ & $\begin{array}{r}0.8 \\
5 \\
\end{array}$ & $\begin{array}{r}1860 . \\
1 \\
\end{array}$ & 39.1 & 1880.1 & 24.3 & $\begin{array}{r}1902 . \\
3 \\
\end{array}$ & 26.6 & 1902.3 & 26.6 & 97.8 \\
\hline $\begin{array}{l}\text { BU0710- } \\
69\end{array}$ & $\begin{array}{r}206 \\
1 \\
\end{array}$ & $\begin{array}{r}15785 \\
5 \\
\end{array}$ & 2.8 & $\begin{array}{r}8.580 \\
7 \\
\end{array}$ & 1.6 & 4.8913 & 3.4 & $\begin{array}{r}0.304 \\
4 \\
\end{array}$ & $\begin{array}{c}3 . \\
0\end{array}$ & $\begin{array}{r}0.8 \\
9 \\
\end{array}$ & $\begin{array}{r}1713 . \\
1 \\
\end{array}$ & 45.4 & 1800.8 & 28.7 & $\begin{array}{r}1903 . \\
8 \\
\end{array}$ & 28.2 & 1903.8 & 28.2 & 90.0 \\
\hline $\begin{array}{l}\text { BU0710- } \\
41\end{array}$ & 661 & $\begin{array}{r}28489 \\
5 \\
\end{array}$ & 2.5 & $\begin{array}{r}8.543 \\
4 \\
\end{array}$ & 3.9 & 5.5544 & 4.0 & $\begin{array}{r}0.344 \\
2 \\
\end{array}$ & $\begin{array}{r}1 . \\
0\end{array}$ & $\begin{array}{r}0.2 \\
5 \\
\end{array}$ & $\begin{array}{r}1906 . \\
7 \\
\end{array}$ & 16.5 & 1909.1 & 34.4 & $\begin{array}{r}1911 . \\
6 \\
\end{array}$ & 69.5 & 1911.6 & 69.5 & 99.7 \\
\hline $\begin{array}{l}\text { BU0710- } \\
90\end{array}$ & 653 & $\begin{array}{r}22953 \\
0 \\
\end{array}$ & 2.1 & $\begin{array}{r}8.532 \\
9 \\
\end{array}$ & 1.6 & 5.4046 & 2.0 & $\begin{array}{r}0.334 \\
5 \\
\end{array}$ & $\begin{array}{r}1 . \\
2\end{array}$ & $\begin{array}{r}0.6 \\
1\end{array}$ & $\begin{array}{r}1860 . \\
0\end{array}$ & 19.6 & 1885.6 & 17.0 & $\begin{array}{r}1913 . \\
8 \\
\end{array}$ & 28.2 & 1913.8 & 28.2 & 97.2 \\
\hline $\begin{array}{l}\text { BU0710- } \\
89\end{array}$ & 557 & $\begin{array}{r}12102 \\
0 \\
\end{array}$ & 1.2 & $\begin{array}{r}8.525 \\
4 \\
\end{array}$ & 1.6 & 5.5688 & 2.0 & $\begin{array}{r}0.344 \\
3 \\
\end{array}$ & $\begin{array}{r}1 . \\
2 \\
\end{array}$ & $\begin{array}{r}0.6 \\
1\end{array}$ & $\begin{array}{r}1907 . \\
5 \\
\end{array}$ & 20.0 & 1911.3 & 17.1 & $\begin{array}{r}1915 . \\
4 \\
\end{array}$ & 28.4 & 1915.4 & 28.4 & 99.6 \\
\hline $\begin{array}{l}\text { BU0710- } \\
18\end{array}$ & 87 & 34230 & 1.2 & $\begin{array}{r}8.485 \\
5 \\
\end{array}$ & 3.1 & 5.7916 & 3.4 & $\begin{array}{r}0.356 \\
4 \\
\end{array}$ & $\begin{array}{c}1 . \\
5 \\
\end{array}$ & $\begin{array}{r}0.4 \\
3 \\
\end{array}$ & $\begin{array}{r}1965 . \\
2 \\
\end{array}$ & 25.2 & 1945.2 & 29.7 & $\begin{array}{r}1923 . \\
8 \\
\end{array}$ & 55.4 & 1923.8 & 55.4 & $\begin{array}{r}102 . \\
2 \\
\end{array}$ \\
\hline BU0710-2 & 782 & $\begin{array}{r}12056 \\
0 \\
\end{array}$ & 1.8 & $\begin{array}{r}8.474 \\
0 \\
\end{array}$ & 1.7 & 5.7602 & 2.3 & $\begin{array}{r}0.354 \\
0 \\
\end{array}$ & $\begin{array}{c}1 . \\
6 \\
\end{array}$ & $\begin{array}{r}0.6 \\
8 \\
\end{array}$ & $\begin{array}{r}1953 . \\
8 \\
\end{array}$ & 26.6 & 1940.4 & 20.0 & $\begin{array}{r}1926 . \\
3 \\
\end{array}$ & 30.3 & 1926.3 & 30.3 & $\begin{array}{r}101 . \\
4\end{array}$ \\
\hline $\begin{array}{l}\text { BU0710- } \\
72\end{array}$ & 970 & $\begin{array}{r}32572 \\
0 \\
\end{array}$ & 8.4 & $\begin{array}{r}8.461 \\
5 \\
\end{array}$ & 1.1 & 5.6017 & 2.1 & $\begin{array}{r}0.343 \\
8 \\
\end{array}$ & $\begin{array}{r}1 . \\
8 \\
\end{array}$ & $\begin{array}{r}0.8 \\
4 \\
\end{array}$ & $\begin{array}{r}1904 . \\
8 \\
\end{array}$ & 29.2 & 1916.4 & 18.1 & $\begin{array}{r}1928 . \\
9 \\
\end{array}$ & 20.4 & 1928.9 & 20.4 & 98.7 \\
\hline $\begin{array}{l}\text { BU0710- } \\
100\end{array}$ & 560 & $\begin{array}{r}21328 \\
5 \\
\end{array}$ & 2.7 & $\begin{array}{r}8.457 \\
1 \\
\end{array}$ & 2.1 & 5.4670 & 5.3 & $\begin{array}{r}0.335 \\
3 \\
\end{array}$ & $\begin{array}{r}4 . \\
9 \\
\end{array}$ & $\begin{array}{r}0.9 \\
2 \\
\end{array}$ & $\begin{array}{r}1864 . \\
2 \\
\end{array}$ & 79.3 & 1895.4 & 45.9 & $\begin{array}{r}1929 . \\
8\end{array}$ & 38.2 & 1929.8 & 38.2 & 96.6 \\
\hline $\begin{array}{l}\text { BU0710- } \\
35\end{array}$ & 679 & $\begin{array}{r}18709 \\
0 \\
\end{array}$ & 16.3 & $\begin{array}{r}8.405 \\
7 \\
\end{array}$ & 2.0 & 5.5274 & 2.8 & $\begin{array}{r}0.337 \\
0 \\
\end{array}$ & $\begin{array}{r}1 . \\
9 \\
\end{array}$ & $\begin{array}{r}0.6 \\
9 \\
\end{array}$ & $\begin{array}{r}1872 . \\
1 \\
\end{array}$ & 30.7 & 1904.9 & 23.7 & $\begin{array}{r}1940 . \\
8 \\
\end{array}$ & 35.8 & 1940.8 & 35.8 & 96.5 \\
\hline BU0710- & 763 & 26910 & 3.4 & 8.378 & 1.5 & 5.8025 & 2.1 & 0.352 & 1. & 0.6 & 1946. & 23.9 & 1946.8 & 17.8 & 1946. & 26.7 & 1946.7 & 26.7 & 100. \\
\hline
\end{tabular}




\begin{tabular}{|c|c|c|c|c|c|c|c|c|c|c|c|c|c|c|c|c|c|c|c|}
\hline 12 & & & & 0 & & & & 6 & 4 & 9 & 9 & & & & 7 & & & & 0 \\
\hline $\begin{array}{l}\text { BU0710- } \\
53\end{array}$ & 420 & $\begin{array}{r}21393 \\
5\end{array}$ & 1.5 & $\begin{array}{r}8.359 \\
9\end{array}$ & 3.1 & 5.6542 & 3.4 & $\begin{array}{r}0.342 \\
8\end{array}$ & $\begin{array}{r}1 . \\
2\end{array}$ & $\begin{array}{r}0.3 \\
5\end{array}$ & $\begin{array}{r}1900 . \\
2\end{array}$ & 19.6 & 1924.4 & 29.0 & $\begin{array}{r}1950 . \\
5\end{array}$ & 56.1 & 1950.5 & 56.1 & 97.4 \\
\hline $\begin{array}{l}\text { BU0710- } \\
63\end{array}$ & $\begin{array}{r}169 \\
0\end{array}$ & 21620 & 3.2 & $\begin{array}{r}8.357 \\
9\end{array}$ & 3.5 & 5.4737 & 3.9 & $\begin{array}{r}0.331 \\
8\end{array}$ & $\begin{array}{l}1 . \\
7\end{array}$ & $\begin{array}{r}0.4 \\
4\end{array}$ & $\begin{array}{r}1847 . \\
1\end{array}$ & 27.3 & 1896.5 & 33.4 & $\begin{array}{r}1950 . \\
9\end{array}$ & 62.6 & 1950.9 & 62.6 & 94.7 \\
\hline $\begin{array}{l}\text { BU0710- } \\
42\end{array}$ & $\begin{array}{r}174 \\
0\end{array}$ & $\begin{array}{r}23136 \\
5\end{array}$ & 4.4 & $\begin{array}{r}8.345 \\
4\end{array}$ & 2.8 & 5.6147 & 3.5 & $\begin{array}{r}0.339 \\
8\end{array}$ & $\begin{array}{r}2 . \\
1\end{array}$ & $\begin{array}{r}0.6 \\
0\end{array}$ & $\begin{array}{r}1885 . \\
9\end{array}$ & 34.3 & 1918.4 & 30.0 & $\begin{array}{r}1953 . \\
6\end{array}$ & 49.5 & 1953.6 & 49.5 & 96.5 \\
\hline BU0710-8 & 295 & $\begin{array}{r}12620 \\
0\end{array}$ & 3.9 & $\begin{array}{r}8.336 \\
3\end{array}$ & 2.5 & 5.8955 & 3.3 & $\begin{array}{r}0.356 \\
4\end{array}$ & $\begin{array}{r}2 . \\
0\end{array}$ & $\begin{array}{r}0.6 \\
3\end{array}$ & $\begin{array}{r}1965 . \\
3\end{array}$ & 34.6 & 1960.6 & 28.3 & $\begin{array}{r}1955 . \\
6\end{array}$ & 45.4 & 1955.6 & 45.4 & $\begin{array}{r}100 . \\
5\end{array}$ \\
\hline $\begin{array}{l}\text { BU0710- } \\
70\end{array}$ & 213 & 73955 & 0.8 & $\begin{array}{r}8.329 \\
6 \\
\end{array}$ & 1.6 & 5.8260 & 2.5 & $\begin{array}{r}0.352 \\
0 \\
\end{array}$ & $\begin{array}{r}2 . \\
0 \\
\end{array}$ & $\begin{array}{r}0.7 \\
8 \\
\end{array}$ & $\begin{array}{r}1944 . \\
0 \\
\end{array}$ & 32.7 & 1950.3 & 21.6 & $\begin{array}{r}1957 . \\
0 \\
\end{array}$ & 27.9 & 1957.0 & 27.9 & 99.3 \\
\hline $\begin{array}{l}\text { BU0710- } \\
59\end{array}$ & 424 & $\begin{array}{r}15212 \\
0\end{array}$ & 12.7 & $\begin{array}{r}8.311 \\
0\end{array}$ & 3.2 & 6.0545 & 3.5 & $\begin{array}{r}0.364 \\
9\end{array}$ & $\begin{array}{l}1 . \\
4\end{array}$ & $\begin{array}{r}0.4 \\
1 \\
\end{array}$ & $\begin{array}{r}2005 . \\
6 \\
\end{array}$ & 24.5 & 1983.7 & 30.1 & $\begin{array}{r}1961 . \\
0 \\
\end{array}$ & 56.2 & 1961.0 & 56.2 & $\begin{array}{r}102 . \\
3 \\
\end{array}$ \\
\hline $\begin{array}{l}\text { BU0710- } \\
25\end{array}$ & $\begin{array}{r}112 \\
3\end{array}$ & $\begin{array}{r}34541 \\
5\end{array}$ & 3.9 & $\begin{array}{r}8.294 \\
0\end{array}$ & 4.5 & 5.6987 & 5.2 & $\begin{array}{r}0.342 \\
8\end{array}$ & $\begin{array}{r}2 . \\
6\end{array}$ & $\begin{array}{r}0.5 \\
1\end{array}$ & $\begin{array}{r}1900 . \\
1\end{array}$ & 43.4 & 1931.2 & 44.9 & $\begin{array}{r}1964 . \\
7\end{array}$ & 80.0 & 1964.7 & 80.0 & 96.7 \\
\hline $\begin{array}{l}\text { BU0710- } \\
11\end{array}$ & 362 & 35435 & 2.2 & $\begin{array}{r}8.231 \\
7 \\
\end{array}$ & 5.7 & 5.3087 & 6.3 & $\begin{array}{r}0.316 \\
9 \\
\end{array}$ & $\begin{array}{r}2 . \\
6\end{array}$ & $\begin{array}{r}0.4 \\
1 \\
\end{array}$ & $\begin{array}{r}1774 . \\
8\end{array}$ & 40.2 & 1870.3 & 53.6 & $\begin{array}{r}1978 . \\
1\end{array}$ & $\begin{array}{r}101 . \\
8 \\
\end{array}$ & 1978.1 & $\begin{array}{r}101 . \\
8 \\
\end{array}$ & 89.7 \\
\hline $\begin{array}{l}\text { BU0710- } \\
67\end{array}$ & 372 & 85835 & 1.1 & $\begin{array}{r}8.138 \\
8\end{array}$ & 8.4 & 5.4579 & 9.1 & $\begin{array}{r}0.322 \\
2\end{array}$ & $\begin{array}{r}3 . \\
6\end{array}$ & $\begin{array}{r}0.3 \\
9\end{array}$ & $\begin{array}{r}1800 . \\
3\end{array}$ & 55.8 & 1894.0 & 78.7 & $\begin{array}{r}1998 . \\
3\end{array}$ & $\begin{array}{r}150 . \\
1 \\
\end{array}$ & 1998.3 & $\begin{array}{r}150 . \\
1 \\
\end{array}$ & 90.1 \\
\hline $\begin{array}{l}\text { BU0710- } \\
56\end{array}$ & 214 & $\begin{array}{r}10857 \\
5 \\
\end{array}$ & 2.1 & $\begin{array}{r}7.892 \\
2 \\
\end{array}$ & 2.7 & 6.4296 & 3.2 & $\begin{array}{r}0.368 \\
0 \\
\end{array}$ & $\begin{array}{r}1 . \\
6\end{array}$ & $\begin{array}{r}0.5 \\
2 \\
\end{array}$ & $\begin{array}{r}2020 . \\
1\end{array}$ & 28.4 & 2036.3 & 27.7 & $\begin{array}{r}2052 . \\
8 \\
\end{array}$ & 47.5 & 2052.8 & 47.5 & 98.4 \\
\hline $\begin{array}{l}\text { BU0710- } \\
27\end{array}$ & 734 & $\begin{array}{r}27736 \\
0\end{array}$ & 4.5 & $\begin{array}{r}7.879 \\
3\end{array}$ & 3.3 & 6.5611 & 3.6 & $\begin{array}{r}0.374 \\
9\end{array}$ & $\begin{array}{l}1 . \\
4\end{array}$ & $\begin{array}{r}0.3 \\
9\end{array}$ & $\begin{array}{r}2052 . \\
6\end{array}$ & 24.8 & 2054.1 & 31.8 & $\begin{array}{r}2055 . \\
7\end{array}$ & 58.6 & 2055.7 & 58.6 & 99.9 \\
\hline $\begin{array}{l}\text { BU0710- } \\
49\end{array}$ & 521 & $\begin{array}{r}11679 \\
0\end{array}$ & 2.7 & $\begin{array}{r}7.761 \\
4\end{array}$ & 3.8 & 6.5173 & 3.9 & $\begin{array}{r}0.366 \\
9\end{array}$ & $\begin{array}{l}1 . \\
0\end{array}$ & $\begin{array}{r}0.2 \\
6\end{array}$ & $\begin{array}{r}2014 . \\
6\end{array}$ & 17.3 & 2048.2 & 34.3 & $\begin{array}{r}2082 . \\
2\end{array}$ & 66.4 & 2082.2 & 66.4 & 96.8 \\
\hline $\begin{array}{l}\text { BU0710- } \\
26\end{array}$ & 584 & $\begin{array}{r}34044 \\
0\end{array}$ & 5.8 & $\begin{array}{r}7.664 \\
6\end{array}$ & 2.1 & 6.9239 & 2.5 & $\begin{array}{r}0.384 \\
9\end{array}$ & $\begin{array}{r}1 . \\
4\end{array}$ & $\begin{array}{r}0.5 \\
4\end{array}$ & $\begin{array}{r}2099 . \\
1\end{array}$ & 24.4 & 2101.7 & 22.5 & $\begin{array}{r}2104 . \\
3\end{array}$ & 37.6 & 2104.3 & 37.6 & 99.8 \\
\hline BU0710-9 & 384 & $\begin{array}{r}20250 \\
0 \\
\end{array}$ & 3.7 & $\begin{array}{r}7.645 \\
4 \\
\end{array}$ & 1.2 & 6.9849 & 1.9 & $\begin{array}{r}0.387 \\
3 \\
\end{array}$ & $\begin{array}{r}1 . \\
4\end{array}$ & $\begin{array}{r}0.7 \\
6 \\
\end{array}$ & $\begin{array}{r}2110 . \\
3\end{array}$ & 25.9 & 2109.5 & 16.8 & $\begin{array}{r}2108 . \\
7 \\
\end{array}$ & 21.6 & 2108.7 & 21.6 & $\begin{array}{r}100 . \\
1\end{array}$ \\
\hline $\begin{array}{l}\text { BU0710- } \\
68\end{array}$ & $\begin{array}{r}188 \\
9 \\
\end{array}$ & $\begin{array}{r}21802 \\
5 \\
\end{array}$ & 1.1 & $\begin{array}{r}7.619 \\
6\end{array}$ & 5.0 & 6.0486 & 5.9 & $\begin{array}{r}0.334 \\
3\end{array}$ & $\begin{array}{r}3 . \\
2\end{array}$ & $\begin{array}{r}0.5 \\
4\end{array}$ & $\begin{array}{r}1859 . \\
0\end{array}$ & 50.9 & 1982.9 & 51.2 & $\begin{array}{r}2114 . \\
6\end{array}$ & 87.0 & 2114.6 & 87.0 & 87.9 \\
\hline $\begin{array}{l}\text { BU0710- } \\
29\end{array}$ & 475 & 76165 & 8.1 & $\begin{array}{r}7.613 \\
9\end{array}$ & 4.0 & 5.6503 & 4.2 & $\begin{array}{r}0.312 \\
0\end{array}$ & $\begin{array}{l}1 . \\
4\end{array}$ & $\begin{array}{r}0.3 \\
4\end{array}$ & $\begin{array}{r}1750 . \\
6\end{array}$ & 21.9 & 1923.8 & 36.5 & $\begin{array}{r}2115 . \\
9\end{array}$ & 69.8 & 2115.9 & 69.8 & 82.7 \\
\hline $\begin{array}{l}\text { BU0710- } \\
47\end{array}$ & 651 & $\begin{array}{r}22073 \\
5\end{array}$ & 2.7 & $\begin{array}{r}7.583 \\
4\end{array}$ & 2.9 & 7.0092 & 3.7 & $\begin{array}{r}0.385 \\
5\end{array}$ & $\begin{array}{r}2 . \\
3\end{array}$ & $\begin{array}{r}0.6 \\
3\end{array}$ & $\begin{array}{r}2102 . \\
0\end{array}$ & 42.0 & 2112.6 & 33.2 & $\begin{array}{r}2123 . \\
0\end{array}$ & 51.0 & 2123.0 & 51.0 & 99.0 \\
\hline $\begin{array}{l}\text { BU0710- } \\
43\end{array}$ & 387 & 61150 & 0.9 & $\begin{array}{r}7.294 \\
6\end{array}$ & 2.7 & 6.9761 & 3.4 & $\begin{array}{r}0.369 \\
1\end{array}$ & $\begin{array}{r}2 . \\
1\end{array}$ & $\begin{array}{r}0.6 \\
3\end{array}$ & $\begin{array}{r}2025 . \\
0\end{array}$ & 37.2 & 2108.4 & 30.3 & $\begin{array}{r}2190 . \\
7\end{array}$ & 46.3 & 2190.7 & 46.3 & 92.4 \\
\hline $\begin{array}{l}\text { BU0710- } \\
84\end{array}$ & $\begin{array}{r}111 \\
0 \\
\end{array}$ & $\begin{array}{r}16743 \\
5 \\
\end{array}$ & 12.1 & $\begin{array}{r}7.272 \\
4 \\
\end{array}$ & 2.2 & 7.4952 & 2.5 & $\begin{array}{r}0.395 \\
3\end{array}$ & $\begin{array}{r}1 . \\
2\end{array}$ & $\begin{array}{r}0.4 \\
9\end{array}$ & $\begin{array}{r}2147 . \\
5\end{array}$ & 22.3 & 2172.4 & 22.5 & $\begin{array}{r}2196 . \\
0\end{array}$ & 38.1 & 2196.0 & 38.1 & 97.8 \\
\hline $\begin{array}{l}\text { BU0710- } \\
97\end{array}$ & 612 & $\begin{array}{r}16651 \\
5 \\
\end{array}$ & 1.8 & $\begin{array}{r}7.249 \\
7\end{array}$ & 2.3 & 7.5132 & 3.9 & $\begin{array}{r}0.395 \\
0\end{array}$ & $\begin{array}{r}3 . \\
1\end{array}$ & $\begin{array}{r}0.8 \\
0\end{array}$ & $\begin{array}{r}2146 . \\
2\end{array}$ & 56.6 & 2174.6 & 34.6 & $\begin{array}{r}2201 . \\
5\end{array}$ & 39.9 & 2201.5 & 39.9 & 97.5 \\
\hline $\begin{array}{l}\text { BU0710- } \\
31\end{array}$ & $\begin{array}{r}138 \\
0\end{array}$ & $\begin{array}{r}19416 \\
0\end{array}$ & 3.5 & $\begin{array}{r}7.248 \\
5\end{array}$ & 2.2 & 5.6825 & 3.4 & $\begin{array}{r}0.298 \\
7\end{array}$ & $\begin{array}{r}2 . \\
7\end{array}$ & $\begin{array}{r}0.7 \\
8\end{array}$ & $\begin{array}{r}1685 . \\
0\end{array}$ & 39.3 & 1928.7 & 29.5 & $\begin{array}{r}2201 . \\
7\end{array}$ & 37.5 & 2201.7 & 37.5 & 76.5 \\
\hline $\begin{array}{l}\text { BU0710- } \\
48\end{array}$ & 304 & $\begin{array}{r}18569 \\
0\end{array}$ & 2.1 & $\begin{array}{r}7.183 \\
0\end{array}$ & 3.9 & 7.8946 & 4.0 & $\begin{array}{r}0.411 \\
3\end{array}$ & $\begin{array}{l}1 . \\
0\end{array}$ & $\begin{array}{r}0.2 \\
5\end{array}$ & $\begin{array}{r}2220 . \\
7\end{array}$ & 18.8 & 2219.1 & 36.4 & $\begin{array}{r}2217 . \\
5\end{array}$ & 67.8 & 2217.5 & 67.8 & $\begin{array}{r}100 . \\
1\end{array}$ \\
\hline $\begin{array}{l}\text { BU0710- } \\
14\end{array}$ & 213 & $\begin{array}{r}11321 \\
0\end{array}$ & 0.6 & $\begin{array}{r}7.169 \\
0\end{array}$ & 1.9 & 7.5383 & 2.5 & $\begin{array}{r}0.391 \\
9\end{array}$ & $\begin{array}{c}1 . \\
7\end{array}$ & $\begin{array}{r}0.6 \\
8\end{array}$ & $\begin{array}{r}2131 . \\
9\end{array}$ & 31.4 & 2177.6 & 22.7 & $\begin{array}{r}2220 . \\
9\end{array}$ & 32.1 & 2220.9 & 32.1 & 96.0 \\
\hline $\begin{array}{l}\text { BU0710- } \\
32\end{array}$ & 774 & $\begin{array}{r}33328 \\
5\end{array}$ & 2.6 & $\begin{array}{r}7.168 \\
1\end{array}$ & 1.6 & 7.3629 & 2.8 & $\begin{array}{r}0.382 \\
8\end{array}$ & $\begin{array}{r}2 . \\
3\end{array}$ & $\begin{array}{r}0.8 \\
2\end{array}$ & $\begin{array}{r}2089 . \\
3\end{array}$ & 40.7 & 2156.5 & 24.9 & $\begin{array}{r}2221 . \\
1\end{array}$ & 27.6 & 2221.1 & 27.6 & 94.1 \\
\hline
\end{tabular}




\begin{tabular}{|c|c|c|c|c|c|c|c|c|c|c|c|c|c|c|c|c|c|c|c|}
\hline BU0710-1 & 247 & 7045 & 1.2 & $\begin{array}{r}6.999 \\
2 \\
\end{array}$ & 3.4 & 7.5779 & 3.5 & $\begin{array}{r}0.384 \\
7 \\
\end{array}$ & $\begin{array}{r}1 . \\
0\end{array}$ & $\begin{array}{r}0.2 \\
9 \\
\end{array}$ & $\begin{array}{r}2098 . \\
1 \\
\end{array}$ & 17.9 & 2182.3 & 31.5 & $\begin{array}{r}2262 . \\
3 \\
\end{array}$ & 58.0 & 2262.3 & 58.0 & 92.7 \\
\hline $\begin{array}{l}\text { BU0710- } \\
55\end{array}$ & 881 & 55470 & 0.8 & $\begin{array}{r}6.875 \\
2\end{array}$ & 3.0 & 7.6549 & 4.5 & $\begin{array}{r}0.381 \\
7\end{array}$ & $\begin{array}{r}3 . \\
4\end{array}$ & $\begin{array}{r}0.7 \\
4\end{array}$ & $\begin{array}{r}2084 . \\
2\end{array}$ & 59.8 & 2191.3 & 40.6 & $\begin{array}{r}2293 . \\
1\end{array}$ & 51.9 & 2293.1 & 51.9 & 90.9 \\
\hline $\begin{array}{l}\text { BU0710- } \\
21\end{array}$ & 204 & 74505 & 1.2 & $\begin{array}{r}6.765 \\
3\end{array}$ & 1.8 & 8.7625 & 2.6 & $\begin{array}{r}0.429 \\
9\end{array}$ & $\begin{array}{r}1 . \\
9\end{array}$ & $\begin{array}{r}0.7 \\
4\end{array}$ & $\begin{array}{r}2305 . \\
5\end{array}$ & 37.4 & 2313.6 & 23.7 & $\begin{array}{r}2320 . \\
8\end{array}$ & 30.0 & 2320.8 & 30.0 & 99.3 \\
\hline $\begin{array}{l}\text { BU0710- } \\
98\end{array}$ & 342 & 10145 & 1.2 & $\begin{array}{r}6.747 \\
6 \\
\end{array}$ & 6.2 & 8.4367 & 6.5 & $\begin{array}{r}0.412 \\
9 \\
\end{array}$ & $\begin{array}{r}1 . \\
8\end{array}$ & $\begin{array}{r}0.2 \\
8 \\
\end{array}$ & $\begin{array}{r}2228 . \\
1\end{array}$ & 33.7 & 2279.1 & 58.8 & $\begin{array}{r}2325 . \\
3 \\
\end{array}$ & $\begin{array}{r}106 . \\
7\end{array}$ & 2325.3 & $\begin{array}{r}106 . \\
7\end{array}$ & 95.8 \\
\hline $\begin{array}{l}\text { BU0710- } \\
36\end{array}$ & 673 & $\begin{array}{r}22078 \\
0 \\
\end{array}$ & 2.4 & $\begin{array}{r}6.723 \\
5 \\
\end{array}$ & 2.8 & 8.1411 & 5.6 & $\begin{array}{r}0.397 \\
0 \\
\end{array}$ & $\begin{array}{r}4 . \\
9 \\
\end{array}$ & $\begin{array}{r}0.8 \\
7 \\
\end{array}$ & $\begin{array}{r}2155 . \\
2 \\
\end{array}$ & 89.6 & 2246.8 & 51.1 & $\begin{array}{r}2331 . \\
4 \\
\end{array}$ & 48.5 & 2331.4 & 48.5 & 92.4 \\
\hline $\begin{array}{l}\text { BU0710- } \\
52\end{array}$ & 247 & 9775 & 2.0 & $\begin{array}{r}6.723 \\
0 \\
\end{array}$ & 4.9 & 6.6366 & 5.2 & $\begin{array}{r}0.323 \\
6 \\
\end{array}$ & $\begin{array}{r}1 . \\
9\end{array}$ & $\begin{array}{r}0.3 \\
5 \\
\end{array}$ & $\begin{array}{r}1807 . \\
3 \\
\end{array}$ & 29.2 & 2064.2 & 46.3 & $\begin{array}{r}2331 . \\
5 \\
\end{array}$ & 84.0 & 2331.5 & 84.0 & 77.5 \\
\hline $\begin{array}{l}\text { BU0710- } \\
74\end{array}$ & 270 & $\begin{array}{r}10118 \\
0\end{array}$ & 1.1 & $\begin{array}{r}6.722 \\
1\end{array}$ & 2.3 & 8.5056 & 2.5 & $\begin{array}{r}0.414 \\
7\end{array}$ & $\begin{array}{r}1 . \\
2\end{array}$ & $\begin{array}{r}0.4 \\
6\end{array}$ & $\begin{array}{r}2236 . \\
3\end{array}$ & 21.9 & 2286.5 & 23.0 & $\begin{array}{r}2331 . \\
7\end{array}$ & 38.5 & 2331.7 & 38.5 & 95.9 \\
\hline BU0710-4 & 368 & $\begin{array}{r}13618 \\
5 \\
\end{array}$ & 1.7 & $\begin{array}{r}6.667 \\
1 \\
\end{array}$ & 2.2 & 9.2134 & 2.8 & $\begin{array}{r}0.445 \\
5\end{array}$ & $\begin{array}{l}1 . \\
7\end{array}$ & $\begin{array}{r}0.6 \\
2\end{array}$ & $\begin{array}{r}2375 . \\
3\end{array}$ & 34.0 & 2359.4 & 25.5 & $\begin{array}{r}2345 . \\
8\end{array}$ & 37.5 & 2345.8 & 37.5 & $\begin{array}{r}101 . \\
3\end{array}$ \\
\hline $\begin{array}{l}\text { BU0710- } \\
10\end{array}$ & 213 & 98800 & 1.7 & $\begin{array}{r}6.499 \\
4 \\
\end{array}$ & 2.9 & 9.6121 & 3.2 & $\begin{array}{r}0.453 \\
1 \\
\end{array}$ & $\begin{array}{r}1 . \\
4\end{array}$ & $\begin{array}{r}0.4 \\
5 \\
\end{array}$ & $\begin{array}{r}2409 . \\
0 \\
\end{array}$ & 28.7 & 2398.3 & 29.3 & $\begin{array}{r}2389 . \\
3 \\
\end{array}$ & 48.5 & 2389.3 & 48.5 & $\begin{array}{r}100 . \\
8 \\
\end{array}$ \\
\hline $\begin{array}{l}\text { BU0710- } \\
88\end{array}$ & 527 & $\begin{array}{r}26216 \\
0 \\
\end{array}$ & 1.2 & $\begin{array}{r}6.498 \\
0 \\
\end{array}$ & 1.8 & 9.3919 & 2.0 & $\begin{array}{r}0.442 \\
6 \\
\end{array}$ & $\begin{array}{r}1 . \\
0\end{array}$ & $\begin{array}{r}0.4 \\
9 \\
\end{array}$ & $\begin{array}{r}2362 . \\
4 \\
\end{array}$ & 19.8 & 2377.0 & 18.6 & $\begin{array}{r}2389 . \\
6 \\
\end{array}$ & 30.0 & 2389.6 & 30.0 & 98.9 \\
\hline $\begin{array}{l}\text { BU0710- } \\
87\end{array}$ & $\begin{array}{r}212 \\
6\end{array}$ & $\begin{array}{r}27437 \\
5\end{array}$ & 0.6 & $\begin{array}{r}6.475 \\
6\end{array}$ & 1.3 & 6.8930 & 4.8 & $\begin{array}{r}0.323 \\
7\end{array}$ & $\begin{array}{r}4 . \\
6\end{array}$ & $\begin{array}{r}0.9 \\
6\end{array}$ & $\begin{array}{r}1807 . \\
9\end{array}$ & 72.8 & 2097.8 & 42.6 & $\begin{array}{r}2395 . \\
5\end{array}$ & 22.5 & 2395.5 & 22.5 & 75.5 \\
\hline $\begin{array}{l}\text { BU0710- } \\
82\end{array}$ & 287 & $\begin{array}{r}14383 \\
5\end{array}$ & 3.0 & $\begin{array}{r}6.454 \\
8\end{array}$ & 5.9 & 9.7640 & 6.1 & $\begin{array}{r}0.457 \\
1\end{array}$ & $\begin{array}{r}1 . \\
6\end{array}$ & $\begin{array}{r}0.2 \\
6\end{array}$ & $\begin{array}{r}2426 . \\
7\end{array}$ & 32.6 & 2412.8 & 56.5 & $\begin{array}{r}2401 . \\
0\end{array}$ & $\begin{array}{r}100 . \\
6\end{array}$ & 2401.0 & $\begin{array}{r}100 . \\
6\end{array}$ & $\begin{array}{r}101 . \\
1\end{array}$ \\
\hline BU0710-3 & 237 & 94935 & 1.1 & $\begin{array}{r}6.431 \\
9 \\
\end{array}$ & 1.0 & 9.6447 & 1.9 & $\begin{array}{r}0.449 \\
9 \\
\end{array}$ & $\begin{array}{r}1 . \\
6 \\
\end{array}$ & $\begin{array}{r}0.8 \\
5 \\
\end{array}$ & $\begin{array}{r}2394 . \\
9 \\
\end{array}$ & 31.8 & 2401.4 & 17.3 & $\begin{array}{r}2407 . \\
0 \\
\end{array}$ & 17.0 & 2407.0 & 17.0 & 99.5 \\
\hline $\begin{array}{l}\text { BU0710- } \\
77\end{array}$ & 252 & 93130 & 1.4 & $\begin{array}{r}6.367 \\
1 \\
\end{array}$ & 1.4 & 9.2173 & 2.3 & $\begin{array}{r}0.425 \\
6 \\
\end{array}$ & $\begin{array}{r}1 . \\
9\end{array}$ & $\begin{array}{r}0.8 \\
1 \\
\end{array}$ & $\begin{array}{r}2286 . \\
0 \\
\end{array}$ & 36.0 & 2359.8 & 21.2 & $\begin{array}{r}2424 . \\
2 \\
\end{array}$ & 23.1 & 2424.2 & 23.1 & 94.3 \\
\hline $\begin{array}{l}\text { BU0710- } \\
19\end{array}$ & 200 & 93755 & 1.2 & $\begin{array}{r}6.364 \\
4 \\
\end{array}$ & 2.1 & $\begin{array}{r}10.022 \\
9 \\
\end{array}$ & 2.3 & $\begin{array}{r}0.462 \\
6 \\
\end{array}$ & $\begin{array}{r}1 . \\
0\end{array}$ & $\begin{array}{r}0.4 \\
5 \\
\end{array}$ & $\begin{array}{r}2451 . \\
2 \\
\end{array}$ & 21.2 & 2436.9 & 21.3 & $\begin{array}{r}2424 . \\
9 \\
\end{array}$ & 34.9 & 2424.9 & 34.9 & $\begin{array}{r}101 . \\
1\end{array}$ \\
\hline $\begin{array}{l}\text { BU0710- } \\
57\end{array}$ & 196 & 81610 & 1.0 & $\begin{array}{r}6.361 \\
5\end{array}$ & 3.5 & 9.7368 & 4.9 & $\begin{array}{r}0.449 \\
2\end{array}$ & $\begin{array}{r}3 . \\
5\end{array}$ & $\begin{array}{r}0.7 \\
1\end{array}$ & $\begin{array}{r}2391 . \\
8\end{array}$ & 69.9 & 2410.2 & 45.4 & $\begin{array}{r}2425 . \\
7\end{array}$ & 58.9 & 2425.7 & 58.9 & 98.6 \\
\hline $\begin{array}{l}\text { BU0710- } \\
66\end{array}$ & 979 & $\begin{array}{r}18925 \\
5 \\
\end{array}$ & 2.3 & $\begin{array}{r}6.314 \\
2 \\
\end{array}$ & 2.0 & 9.2735 & 3.6 & $\begin{array}{r}0.424 \\
7 \\
\end{array}$ & $\begin{array}{r}3 . \\
0\end{array}$ & $\begin{array}{r}0.8 \\
3 \\
\end{array}$ & $\begin{array}{r}2281 . \\
7 \\
\end{array}$ & 57.5 & 2365.4 & 32.9 & $\begin{array}{r}2438 . \\
4 \\
\end{array}$ & 33.7 & 2438.4 & 33.7 & 93.6 \\
\hline $\begin{array}{l}\text { BU0710- } \\
64\end{array}$ & $\begin{array}{r}131 \\
2 \\
\end{array}$ & 45835 & 9.2 & $\begin{array}{r}6.302 \\
9 \\
\end{array}$ & 1.7 & 9.7875 & 2.3 & $\begin{array}{r}0.447 \\
4 \\
\end{array}$ & $\begin{array}{r}1 . \\
6 \\
\end{array}$ & $\begin{array}{r}0.6 \\
9 \\
\end{array}$ & $\begin{array}{r}2383 . \\
7 \\
\end{array}$ & 32.1 & 2415.0 & 21.6 & $\begin{array}{r}2441 . \\
4 \\
\end{array}$ & 28.8 & 2441.4 & 28.8 & 97.6 \\
\hline $\begin{array}{l}\text { BU0710- } \\
85\end{array}$ & 271 & $\begin{array}{r}13579 \\
0 \\
\end{array}$ & 2.1 & $\begin{array}{r}6.199 \\
3 \\
\end{array}$ & 1.6 & 9.9540 & 2.1 & $\begin{array}{r}0.447 \\
5 \\
\end{array}$ & $\begin{array}{r}1 . \\
3 \\
\end{array}$ & $\begin{array}{r}0.6 \\
3 \\
\end{array}$ & $\begin{array}{r}2384 . \\
3 \\
\end{array}$ & 26.1 & 2430.5 & 19.2 & $\begin{array}{r}2469 . \\
4 \\
\end{array}$ & 27.4 & 2469.4 & 27.4 & 96.6 \\
\hline $\begin{array}{l}\text { BU0710- } \\
15\end{array}$ & 796 & $\begin{array}{r}11296 \\
5\end{array}$ & 1.4 & $\begin{array}{r}6.095 \\
9\end{array}$ & 2.0 & 9.8645 & 2.3 & $\begin{array}{r}0.436 \\
1\end{array}$ & $\begin{array}{r}1 . \\
1\end{array}$ & $\begin{array}{r}0.4 \\
9\end{array}$ & $\begin{array}{r}2333 . \\
3\end{array}$ & 21.7 & 2422.2 & 20.8 & $\begin{array}{r}2497 . \\
8\end{array}$ & 33.0 & 2497.8 & 33.0 & 93.4 \\
\hline $\begin{array}{l}\text { BU0710- } \\
76\end{array}$ & 915 & $\begin{array}{r}18539 \\
0\end{array}$ & 1.6 & $\begin{array}{r}6.083 \\
5\end{array}$ & 3.1 & 9.3205 & 3.7 & $\begin{array}{r}0.411 \\
2\end{array}$ & $\begin{array}{r}2 . \\
1\end{array}$ & $\begin{array}{r}0.5 \\
7\end{array}$ & $\begin{array}{r}2220 . \\
6\end{array}$ & 39.8 & 2370.0 & 34.1 & $\begin{array}{r}2501 . \\
2\end{array}$ & 51.5 & 2501.2 & 51.5 & 88.8 \\
\hline $\begin{array}{l}\text { BU0710- } \\
75\end{array}$ & 236 & $\begin{array}{r}11440 \\
5 \\
\end{array}$ & 1.5 & $\begin{array}{r}6.081 \\
9 \\
\end{array}$ & 1.5 & $\begin{array}{r}10.389 \\
2 \\
\end{array}$ & 1.9 & $\begin{array}{r}0.458 \\
3 \\
\end{array}$ & $\begin{array}{r}1 . \\
1\end{array}$ & $\begin{array}{r}0.6 \\
0 \\
\end{array}$ & $\begin{array}{r}2431 . \\
9 \\
\end{array}$ & 22.7 & 2470.1 & 17.4 & $\begin{array}{r}2501 . \\
6 \\
\end{array}$ & 25.4 & 2501.6 & 25.4 & 97.2 \\
\hline BU0710-6 & 123 & 47205 & 1.0 & $\begin{array}{r}6.065 \\
1 \\
\end{array}$ & 1.0 & $\begin{array}{r}10.722 \\
5 \\
\end{array}$ & 2.8 & $\begin{array}{r}0.471 \\
7 \\
\end{array}$ & $\begin{array}{r}2 . \\
6 \\
\end{array}$ & $\begin{array}{r}0.9 \\
3 \\
\end{array}$ & $\begin{array}{r}2490 . \\
8 \\
\end{array}$ & 53.9 & 2499.4 & 26.0 & $\begin{array}{r}2506 . \\
3 \\
\end{array}$ & 16.8 & 2506.3 & 16.8 & 99.4 \\
\hline $\begin{array}{l}\text { BU0710- } \\
86\end{array}$ & 57 & 35390 & 1.7 & $\begin{array}{r}6.057 \\
9 \\
\end{array}$ & 1.5 & $\begin{array}{r}10.524 \\
1 \\
\end{array}$ & 2.0 & $\begin{array}{r}0.462 \\
4 \\
\end{array}$ & $\begin{array}{r}1 . \\
4 \\
\end{array}$ & $\begin{array}{r}0.6 \\
8 \\
\end{array}$ & $\begin{array}{r}2450 . \\
1 \\
\end{array}$ & 27.7 & 2482.0 & 18.5 & $\begin{array}{r}2508 . \\
3 \\
\end{array}$ & 24.6 & 2508.3 & 24.6 & 97.7 \\
\hline BU0710- & 498 & 32015 & 1.4 & 6.043 & 4.1 & 9.4548 & 4.3 & 0.414 & 1. & 0.3 & 2235. & 26.3 & 2383.2 & 39.4 & 2512. & 68.3 & 2512.3 & 68.3 & 89.0 \\
\hline
\end{tabular}




\begin{tabular}{|c|c|c|c|c|c|c|c|c|c|c|c|c|c|c|c|c|c|c|c|}
\hline 54 & & & & 5 & & & & 4 & 4 & 2 & 1 & & & & 3 & & & & \\
\hline $\begin{array}{l}\text { BU0710- } \\
20\end{array}$ & 561 & $\begin{array}{r}16675 \\
0 \\
\end{array}$ & 1.6 & $\begin{array}{r}6.008 \\
8 \\
\end{array}$ & 2.8 & $\begin{array}{r}10.764 \\
0 \\
\end{array}$ & 3.3 & $\begin{array}{r}0.469 \\
1 \\
\end{array}$ & $\begin{array}{l}1 . \\
6\end{array}$ & $\begin{array}{r}0.5 \\
0\end{array}$ & $\begin{array}{r}2479 . \\
6 \\
\end{array}$ & 33.3 & 2503.0 & 30.3 & $\begin{array}{r}2522 . \\
0\end{array}$ & 47.5 & 2522.0 & 47.5 & 98.3 \\
\hline $\begin{array}{l}\text { BU0710- } \\
24\end{array}$ & 658 & $\begin{array}{r}11455 \\
5 \\
\end{array}$ & 0.9 & $\begin{array}{r}6.003 \\
3 \\
\end{array}$ & 3.1 & $\begin{array}{r}10.438 \\
9 \\
\end{array}$ & 3.2 & $\begin{array}{r}0.454 \\
5 \\
\end{array}$ & $\begin{array}{r}1 . \\
0\end{array}$ & $\begin{array}{r}0.3 \\
1 \\
\end{array}$ & $\begin{array}{r}2415 . \\
3 \\
\end{array}$ & 20.1 & 2474.5 & 29.8 & $\begin{array}{r}2523 . \\
5 \\
\end{array}$ & 51.2 & 2523.5 & 51.2 & 95.7 \\
\hline $\begin{array}{l}\text { BU0710- } \\
30\end{array}$ & 488 & $\begin{array}{r}21260 \\
5 \\
\end{array}$ & 1.3 & $\begin{array}{r}6.001 \\
3 \\
\end{array}$ & 3.3 & $\begin{array}{r}10.683 \\
3 \\
\end{array}$ & 3.7 & $\begin{array}{r}0.465 \\
0 \\
\end{array}$ & $\begin{array}{r}1 . \\
5\end{array}$ & $\begin{array}{r}0.4 \\
2 \\
\end{array}$ & $\begin{array}{r}2461 . \\
6 \\
\end{array}$ & 31.1 & 2496.0 & 33.9 & $\begin{array}{r}2524 . \\
1 \\
\end{array}$ & 55.8 & 2524.1 & 55.8 & 97.5 \\
\hline $\begin{array}{l}\text { BU0710- } \\
16\end{array}$ & 312 & $\begin{array}{r}12583 \\
0 \\
\end{array}$ & 2.6 & $\begin{array}{r}5.993 \\
3 \\
\end{array}$ & 2.9 & $\begin{array}{r}10.800 \\
2 \\
\end{array}$ & 3.3 & $\begin{array}{r}0.469 \\
5 \\
\end{array}$ & $\begin{array}{r}1 . \\
6 \\
\end{array}$ & $\begin{array}{r}0.4 \\
9 \\
\end{array}$ & $\begin{array}{r}2481 . \\
2 \\
\end{array}$ & 33.8 & 2506.1 & 31.1 & $\begin{array}{r}2526 . \\
3 \\
\end{array}$ & 49.0 & 2526.3 & 49.0 & 98.2 \\
\hline $\begin{array}{l}\text { BU0710- } \\
65\end{array}$ & 414 & 58770 & 1.2 & $\begin{array}{r}5.957 \\
6 \\
\end{array}$ & 4.2 & 9.4717 & 7.3 & $\begin{array}{r}0.409 \\
3 \\
\end{array}$ & $\begin{array}{r}6 . \\
0 \\
\end{array}$ & $\begin{array}{r}0.8 \\
2 \\
\end{array}$ & $\begin{array}{r}2211 . \\
5 \\
\end{array}$ & $\begin{array}{r}111 . \\
8 \\
\end{array}$ & 2384.8 & 67.2 & $\begin{array}{r}2536 . \\
3 \\
\end{array}$ & 70.8 & 2536.3 & 70.8 & 87.2 \\
\hline $\begin{array}{l}\text { BU0710- } \\
33\end{array}$ & 69 & 20320 & 0.7 & $\begin{array}{r}5.890 \\
9 \\
\end{array}$ & 2.7 & $\begin{array}{r}11.093 \\
4 \\
\end{array}$ & 3.0 & $\begin{array}{r}0.474 \\
0 \\
\end{array}$ & $\begin{array}{r}. \\
4\end{array}$ & $\begin{array}{r}0.4 \\
6 \\
\end{array}$ & $\begin{array}{r}2500 . \\
9 \\
\end{array}$ & 29.2 & 2531.0 & 28.3 & $\begin{array}{r}2555 . \\
2 \\
\end{array}$ & 45.1 & 2555.2 & 45.1 & 97.9 \\
\hline $\begin{array}{l}\text { BU0710- } \\
81\end{array}$ & 638 & $\begin{array}{r}21409 \\
5 \\
\end{array}$ & 1.3 & $\begin{array}{r}5.849 \\
1 \\
\end{array}$ & 1.6 & $\begin{array}{r}11.200 \\
4\end{array}$ & 2.1 & $\begin{array}{r}0.475 \\
1 \\
\end{array}$ & $\begin{array}{r}1 . \\
4\end{array}$ & $\begin{array}{r}0.6 \\
6 \\
\end{array}$ & $\begin{array}{r}2506 . \\
1 \\
\end{array}$ & 29.1 & 2540.0 & 19.9 & $\begin{array}{r}2567 . \\
1 \\
\end{array}$ & 26.9 & 2567.1 & 26.9 & 97.6 \\
\hline $\begin{array}{l}\text { BU0710- } \\
50\end{array}$ & $\begin{array}{r}123 \\
6 \\
\end{array}$ & $\begin{array}{r}52841 \\
0 \\
\end{array}$ & 1.9 & $\begin{array}{r}5.830 \\
5 \\
\end{array}$ & 2.2 & $\begin{array}{r}11.687 \\
0 \\
\end{array}$ & 2.4 & $\begin{array}{r}0.494 \\
2 \\
\end{array}$ & $\begin{array}{l}1 . \\
0\end{array}$ & $\begin{array}{r}0.4 \\
2 \\
\end{array}$ & $\begin{array}{r}2588 . \\
8 \\
\end{array}$ & 21.3 & 2579.7 & 22.3 & $\begin{array}{r}2572 . \\
5 \\
\end{array}$ & 36.1 & 2572.5 & 36.1 & $\begin{array}{r}100 . \\
6 \\
\end{array}$ \\
\hline BU0710-5 & 258 & $\begin{array}{r}14525 \\
0 \\
\end{array}$ & 0.7 & $\begin{array}{r}5.820 \\
9 \\
\end{array}$ & 2.1 & $\begin{array}{r}11.605 \\
6 \\
\end{array}$ & 2.6 & $\begin{array}{r}0.490 \\
0 \\
\end{array}$ & $\begin{array}{l}1 . \\
7\end{array}$ & $\begin{array}{r}0.6 \\
3 \\
\end{array}$ & $\begin{array}{r}2570 . \\
5 \\
\end{array}$ & 35.0 & 2573.1 & 24.7 & $\begin{array}{r}2575 . \\
2 \\
\end{array}$ & 34.4 & 2575.2 & 34.4 & 99.8 \\
\hline $\begin{array}{l}\text { BU0710- } \\
37\end{array}$ & 701 & $\begin{array}{r}24542 \\
5 \\
\end{array}$ & 1.6 & $\begin{array}{r}5.791 \\
8 \\
\end{array}$ & 1.7 & $\begin{array}{r}10.431 \\
2 \\
\end{array}$ & 3.1 & $\begin{array}{r}0.438 \\
2 \\
\end{array}$ & $\begin{array}{r}2 . \\
6\end{array}$ & $\begin{array}{r}0.8 \\
4 \\
\end{array}$ & $\begin{array}{r}2342 . \\
4\end{array}$ & 51.1 & 2473.8 & 28.8 & $\begin{array}{r}2583 . \\
6 \\
\end{array}$ & 28.6 & 2583.6 & 28.6 & 90.7 \\
\hline $\begin{array}{l}\text { BU0710- } \\
28\end{array}$ & 99 & 24125 & 1.9 & $\begin{array}{r}5.787 \\
8 \\
\end{array}$ & 7.0 & $\begin{array}{r}10.776 \\
0 \\
\end{array}$ & 7.0 & $\begin{array}{r}0.452 \\
3 \\
\end{array}$ & $\begin{array}{r}1 . \\
1\end{array}$ & $\begin{array}{r}0.1 \\
5 \\
\end{array}$ & $\begin{array}{r}2405 . \\
7 \\
\end{array}$ & 21.1 & 2504.0 & 65.4 & $\begin{array}{r}2584 . \\
7 \\
\end{array}$ & $\begin{array}{r}116 . \\
2 \\
\end{array}$ & 2584.7 & $\begin{array}{r}116 . \\
2 \\
\end{array}$ & 93.1 \\
\hline $\begin{array}{l}\text { BU0710- } \\
44\end{array}$ & 28 & 9105 & 0.8 & $\begin{array}{r}5.653 \\
1 \\
\end{array}$ & 2.6 & $\begin{array}{r}11.590 \\
6 \\
\end{array}$ & 3.2 & $\begin{array}{r}0.475 \\
2 \\
\end{array}$ & $\begin{array}{r}1 . \\
8\end{array}$ & $\begin{array}{r}0.5 \\
7 \\
\end{array}$ & $\begin{array}{r}2506 . \\
4 \\
\end{array}$ & 37.8 & 2571.9 & 29.8 & $\begin{array}{r}2624 . \\
0 \\
\end{array}$ & 43.5 & 2624.0 & 43.5 & 95.5 \\
\hline $\begin{array}{l}\text { BU0710- } \\
83\end{array}$ & 191 & 68060 & 0.5 & $\begin{array}{r}5.474 \\
3 \\
\end{array}$ & 2.0 & $\begin{array}{r}11.437 \\
0 \\
\end{array}$ & 2.4 & $\begin{array}{r}0.454 \\
1 \\
\end{array}$ & $\begin{array}{r}1 . \\
2 \\
\end{array}$ & $\begin{array}{r}0.5 \\
2 \\
\end{array}$ & $\begin{array}{r}2413 . \\
4 \\
\end{array}$ & 24.8 & 2559.4 & 22.0 & $\begin{array}{r}2677 . \\
3 \\
\end{array}$ & 33.3 & 2677.3 & 33.3 & 90.1 \\
\hline $\begin{array}{l}\text { BU0710- } \\
71\end{array}$ & 272 & $\begin{array}{r}16269 \\
5 \\
\end{array}$ & 1.1 & $\begin{array}{r}5.165 \\
4 \\
\end{array}$ & 1.4 & $\begin{array}{r}14.099 \\
0 \\
\end{array}$ & 2.1 & $\begin{array}{r}0.528 \\
2 \\
\end{array}$ & $\begin{array}{r}1 . \\
5\end{array}$ & $\begin{array}{r}0.7 \\
4 \\
\end{array}$ & $\begin{array}{r}2733 . \\
8 \\
\end{array}$ & 34.3 & 2756.4 & 19.6 & $\begin{array}{r}2773 . \\
0 \\
\end{array}$ & 22.6 & 2773.0 & 22.6 & 98.6 \\
\hline $\begin{array}{l}\text { BU0710- } \\
22\end{array}$ & 169 & 4745 & 1.7 & $\begin{array}{r}5.128 \\
7\end{array}$ & 5.6 & $\begin{array}{r}14.104 \\
8 \\
\end{array}$ & 6.2 & $\begin{array}{r}0.524 \\
7\end{array}$ & $\begin{array}{r}2 . \\
6\end{array}$ & $\begin{array}{r}0.4 \\
2\end{array}$ & $\begin{array}{r}2718 . \\
9\end{array}$ & 57.0 & 2756.8 & 58.5 & $\begin{array}{r}2784 . \\
6\end{array}$ & 91.9 & 2784.6 & 91.9 & 97.6 \\
\hline $\begin{array}{l}\text { BU0710- } \\
38\end{array}$ & 192 & $\begin{array}{r}11221 \\
5 \\
\end{array}$ & 1.8 & $\begin{array}{r}5.022 \\
0 \\
\end{array}$ & 2.1 & $\begin{array}{r}14.468 \\
4 \\
\end{array}$ & 2.6 & $\begin{array}{r}0.527 \\
0 \\
\end{array}$ & $\begin{array}{r}1 . \\
6 \\
\end{array}$ & $\begin{array}{r}0.6 \\
0 \\
\end{array}$ & $\begin{array}{r}2728 . \\
7 \\
\end{array}$ & 34.9 & 2780.9 & 24.8 & $\begin{array}{r}2819 . \\
0 \\
\end{array}$ & 34.1 & 2819.0 & 34.1 & 96.8 \\
\hline $\begin{array}{l}\text { BU0710- } \\
78\end{array}$ & 278 & $\begin{array}{r}18050 \\
0 \\
\end{array}$ & 1.7 & $\begin{array}{r}4.969 \\
7 \\
\end{array}$ & 2.1 & $\begin{array}{r}14.812 \\
8 \\
\end{array}$ & 3.7 & $\begin{array}{r}0.533 \\
9 \\
\end{array}$ & $\begin{array}{r}3 . \\
1 \\
\end{array}$ & $\begin{array}{r}0.8 \\
3 \\
\end{array}$ & $\begin{array}{r}2757 . \\
9 \\
\end{array}$ & 69.3 & 2803.3 & 35.3 & $\begin{array}{r}2836 . \\
1 \\
\end{array}$ & 33.6 & 2836.1 & 33.6 & 97.2 \\
\hline $\begin{array}{l}\text { BU0710- } \\
45\end{array}$ & $\begin{array}{r}104 \\
5 \\
\end{array}$ & $\begin{array}{r}16425 \\
5 \\
\end{array}$ & 9.0 & $\begin{array}{r}4.656 \\
4 \\
\end{array}$ & $\begin{array}{r}12 . \\
4\end{array}$ & $\begin{array}{r}15.806 \\
5 \\
\end{array}$ & 12.5 & $\begin{array}{r}0.533 \\
8 \\
\end{array}$ & $\begin{array}{r}1 . \\
8 \\
\end{array}$ & $\begin{array}{r}0.1 \\
4 \\
\end{array}$ & $\begin{array}{r}2757 . \\
4 \\
\end{array}$ & 39.3 & 2865.2 & $\begin{array}{r}120 . \\
1 \\
\end{array}$ & $\begin{array}{r}2941 . \\
8 \\
\end{array}$ & $\begin{array}{r}201 . \\
2 \\
\end{array}$ & 2941.8 & $\begin{array}{r}201 . \\
2 \\
\end{array}$ & 93.7 \\
\hline $\begin{array}{l}\text { BU0710- } \\
23\end{array}$ & 113 & $\begin{array}{r}11171 \\
5 \\
\end{array}$ & 1.4 & $\begin{array}{r}4.070 \\
3 \\
\end{array}$ & 4.3 & $\begin{array}{r}20.678 \\
8 \\
\end{array}$ & 4.5 & $\begin{array}{r}0.610 \\
5\end{array}$ & $\begin{array}{r}1 . \\
3\end{array}$ & $\begin{array}{r}0.2 \\
9 \\
\end{array}$ & $\begin{array}{r}3071 . \\
8\end{array}$ & 31.5 & 3123.7 & 43.1 & $\begin{array}{r}3157 . \\
1\end{array}$ & 67.6 & 3157.1 & 67.6 & 97.3 \\
\hline $\begin{array}{l}\text { BU0722- } \\
73\end{array}$ & $\begin{array}{r}140 \\
7\end{array}$ & 26648 & 1.3 & $\begin{array}{r}16.74 \\
86\end{array}$ & 1.6 & 0.6108 & 1.9 & $\begin{array}{r}0.074 \\
2\end{array}$ & $\begin{array}{l}1 . \\
0\end{array}$ & $\begin{array}{r}0.5 \\
2\end{array}$ & 461.3 & 4.5 & 484.0 & 7.4 & 592.9 & 35.6 & 461.3 & 4.5 & 77.8 \\
\hline $\begin{array}{l}\text { BU0722- } \\
75\end{array}$ & 418 & 23272 & 0.5 & $\begin{array}{r}16.82 \\
34 \\
\end{array}$ & 4.3 & 0.6286 & 4.4 & $\begin{array}{r}0.076 \\
7 \\
\end{array}$ & $\begin{array}{r}1 . \\
0\end{array}$ & $\begin{array}{r}0.2 \\
3 \\
\end{array}$ & 476.4 & 4.6 & 495.2 & 17.3 & 583.3 & 93.2 & 476.4 & 4.6 & 81.7 \\
\hline $\begin{array}{l}\text { BU0722- } \\
84\end{array}$ & 106 & 11128 & 0.5 & $\begin{array}{r}16.79 \\
43\end{array}$ & 4.4 & 0.6315 & 4.8 & $\begin{array}{r}0.076 \\
9\end{array}$ & $\begin{array}{r}1 . \\
9\end{array}$ & $\begin{array}{r}0.4 \\
1\end{array}$ & 477.7 & 8.9 & 497.0 & 18.7 & 587.0 & 94.5 & 477.7 & 8.9 & 81.4 \\
\hline $\begin{array}{l}\text { BU0722- } \\
82\end{array}$ & 479 & 72564 & 1.2 & $\begin{array}{r}17.45 \\
16 \\
\end{array}$ & 5.3 & 0.6254 & 5.3 & $\begin{array}{r}0.079 \\
2 \\
\end{array}$ & $\begin{array}{r}1 . \\
0\end{array}$ & $\begin{array}{r}0.1 \\
9 \\
\end{array}$ & 491.1 & 4.7 & 493.2 & 20.9 & 503.1 & $\begin{array}{r}115 . \\
6 \\
\end{array}$ & 491.1 & 4.7 & 97.6 \\
\hline
\end{tabular}




\begin{tabular}{|c|c|c|c|c|c|c|c|c|c|c|c|c|c|c|c|c|c|c|c|}
\hline $\begin{array}{l}\text { BU0722- } \\
81\end{array}$ & 312 & 35416 & 0.5 & $\begin{array}{r}16.88 \\
87 \\
\end{array}$ & 1.2 & 0.6589 & 1.7 & $\begin{array}{r}0.080 \\
7 \\
\end{array}$ & $\begin{array}{r}1 . \\
2\end{array}$ & $\begin{array}{r}0.7 \\
2 \\
\end{array}$ & 500.3 & 5.7 & 513.9 & 6.7 & 574.9 & 25.3 & 500.3 & 5.7 & 87.0 \\
\hline $\begin{array}{l}\text { BU0722- } \\
32\end{array}$ & 333 & 30068 & 1.4 & $\begin{array}{r}17.42 \\
49\end{array}$ & 1.6 & 0.6538 & 2.4 & $\begin{array}{r}0.082 \\
6\end{array}$ & $\begin{array}{r}1 . \\
8\end{array}$ & $\begin{array}{r}0.7 \\
5\end{array}$ & 511.8 & 8.8 & 510.8 & 9.5 & 506.5 & 34.6 & 511.8 & 8.8 & $\begin{array}{r}101 . \\
0\end{array}$ \\
\hline $\begin{array}{l}\text { BU0722- } \\
23\end{array}$ & $\begin{array}{r}130 \\
2\end{array}$ & $\begin{array}{r}30690 \\
4\end{array}$ & 8.1 & $\begin{array}{r}17.42 \\
86\end{array}$ & 1.1 & 0.6596 & 1.5 & $\begin{array}{r}0.083 \\
4\end{array}$ & $\begin{array}{r}1 . \\
0\end{array}$ & $\begin{array}{r}0.6 \\
7\end{array}$ & 516.2 & 5.0 & 514.4 & 6.0 & 506.0 & 24.2 & 516.2 & 5.0 & $\begin{array}{r}102 . \\
0\end{array}$ \\
\hline $\begin{array}{l}\text { BU0722- } \\
15\end{array}$ & 214 & 20704 & 0.6 & $\begin{array}{r}17.04 \\
49 \\
\end{array}$ & 1.8 & 0.6817 & 2.3 & $\begin{array}{r}0.084 \\
3 \\
\end{array}$ & $\begin{array}{r}1 . \\
4\end{array}$ & $\begin{array}{r}0.6 \\
3 \\
\end{array}$ & 521.6 & 7.1 & 527.8 & 9.3 & 554.8 & 38.3 & 521.6 & 7.1 & 94.0 \\
\hline $\begin{array}{l}\text { BU0722- } \\
10\end{array}$ & $\begin{array}{r}110 \\
6 \\
\end{array}$ & 72804 & 8.2 & $\begin{array}{r}17.33 \\
45 \\
\end{array}$ & 1.0 & 0.6723 & 1.4 & $\begin{array}{r}0.084 \\
5 \\
\end{array}$ & $\begin{array}{r}1 . \\
0\end{array}$ & $\begin{array}{r}0.7 \\
1 \\
\end{array}$ & 523.1 & 5.0 & 522.1 & 5.8 & 517.9 & 22.0 & 523.1 & 5.0 & $\begin{array}{r}101 . \\
0 \\
\end{array}$ \\
\hline $\begin{array}{l}\text { BU0722- } \\
99\end{array}$ & 366 & 41320 & 1.3 & $\begin{array}{r}16.87 \\
81 \\
\end{array}$ & 1.5 & 0.6955 & 2.0 & $\begin{array}{r}0.085 \\
1 \\
\end{array}$ & $\begin{array}{r}1 . \\
3\end{array}$ & $\begin{array}{r}0.6 \\
5 \\
\end{array}$ & 526.7 & 6.6 & 536.1 & 8.4 & 576.2 & 33.3 & 526.7 & 6.6 & 91.4 \\
\hline $\begin{array}{l}\text { BU0722- } \\
66\end{array}$ & 382 & 32484 & 0.9 & $\begin{array}{r}16.95 \\
90 \\
\end{array}$ & 1.9 & 0.6961 & 2.1 & $\begin{array}{r}0.085 \\
6 \\
\end{array}$ & $\begin{array}{r}1 . \\
0\end{array}$ & $\begin{array}{r}0.4 \\
7 \\
\end{array}$ & 529.6 & 5.1 & 536.5 & 8.8 & 565.8 & 40.5 & 529.6 & 5.1 & 93.6 \\
\hline $\begin{array}{l}\text { BU0722- } \\
55\end{array}$ & 535 & 19300 & 5.9 & $\begin{array}{r}16.39 \\
09 \\
\end{array}$ & 1.1 & 0.7206 & 2.4 & $\begin{array}{r}0.085 \\
7 \\
\end{array}$ & $\begin{array}{r}2 . \\
1\end{array}$ & $\begin{array}{r}0.8 \\
9 \\
\end{array}$ & 529.8 & 10.8 & 551.0 & 10.2 & 639.5 & 23.8 & 529.8 & 10.8 & 82.8 \\
\hline $\begin{array}{l}\text { BU0722- } \\
60\end{array}$ & 994 & 81076 & 3.0 & $\begin{array}{r}16.99 \\
92 \\
\end{array}$ & 1.4 & 0.7443 & 2.8 & $\begin{array}{r}0.091 \\
8 \\
\end{array}$ & $\begin{array}{r}2 . \\
5\end{array}$ & $\begin{array}{r}0.8 \\
7 \\
\end{array}$ & 566.0 & 13.4 & 564.9 & 12.3 & 560.7 & 30.5 & 566.0 & 13.4 & $\begin{array}{r}100 . \\
9 \\
\end{array}$ \\
\hline BU0722-2 & 719 & 51892 & 5.5 & $\begin{array}{r}14.66 \\
73 \\
\end{array}$ & 1.6 & 1.0093 & 2.2 & $\begin{array}{r}0.107 \\
4 \\
\end{array}$ & $\begin{array}{r}1 . \\
5\end{array}$ & $\begin{array}{r}0.6 \\
8 \\
\end{array}$ & 657.4 & 9.1 & 708.5 & 11.0 & 874.0 & 33.0 & 657.4 & 9.1 & 75.2 \\
\hline $\begin{array}{l}\text { BU0722- } \\
103\end{array}$ & $\begin{array}{r}161 \\
0\end{array}$ & 96832 & 8.4 & $\begin{array}{r}14.36 \\
43\end{array}$ & 1.3 & 1.1423 & 2.7 & $\begin{array}{r}0.119 \\
0\end{array}$ & $\begin{array}{r}2 . \\
4\end{array}$ & $\begin{array}{r}0.8 \\
9\end{array}$ & 724.8 & 16.6 & 773.6 & 14.7 & 917.1 & 25.7 & 724.8 & 16.6 & 79.0 \\
\hline $\begin{array}{l}\text { BU0722- } \\
48\end{array}$ & 676 & 31200 & 3.0 & $\begin{array}{r}14.50 \\
99\end{array}$ & 1.6 & 1.1526 & 2.7 & $\begin{array}{r}0.121 \\
3\end{array}$ & $\begin{array}{r}2 . \\
2\end{array}$ & $\begin{array}{r}0.8 \\
0\end{array}$ & 738.0 & 15.1 & 778.5 & 14.7 & 896.3 & 33.0 & 738.0 & 15.1 & 82.3 \\
\hline $\begin{array}{l}\text { BU0722- } \\
97\end{array}$ & 990 & $\begin{array}{r}14153 \\
2 \\
\end{array}$ & 2.7 & $\begin{array}{r}15.43 \\
40 \\
\end{array}$ & 1.2 & 1.0935 & 1.5 & $\begin{array}{r}0.122 \\
4 \\
\end{array}$ & $\begin{array}{r}1 . \\
0\end{array}$ & $\begin{array}{r}0.6 \\
5 \\
\end{array}$ & 744.4 & 7.0 & 750.2 & 8.2 & 767.6 & 24.6 & 744.4 & 7.0 & 97.0 \\
\hline $\begin{array}{l}\text { BU0722- } \\
80\end{array}$ & 842 & $\begin{array}{r}10379 \\
2 \\
\end{array}$ & 6.9 & $\begin{array}{r}13.86 \\
93 \\
\end{array}$ & 1.7 & 1.2465 & 5.0 & $\begin{array}{r}0.125 \\
4 \\
\end{array}$ & $\begin{array}{r}4 . \\
7 \\
\end{array}$ & $\begin{array}{r}0.9 \\
4 \\
\end{array}$ & 761.5 & 34.0 & 821.8 & 28.3 & 988.8 & 33.6 & 761.5 & 34.0 & 77.0 \\
\hline $\begin{array}{l}\text { BU0722- } \\
35\end{array}$ & $\begin{array}{r}161 \\
5 \\
\end{array}$ & 83532 & 4.7 & $\begin{array}{r}14.02 \\
08 \\
\end{array}$ & 1.8 & 1.3084 & 2.1 & $\begin{array}{r}0.133 \\
0 \\
\end{array}$ & $\begin{array}{r}1 . \\
0\end{array}$ & $\begin{array}{r}0.4 \\
9 \\
\end{array}$ & 805.2 & 7.6 & 849.4 & 11.8 & 966.7 & 36.5 & 805.2 & 7.6 & 83.3 \\
\hline $\begin{array}{l}\text { BU0722- } \\
61\end{array}$ & $\begin{array}{r}110 \\
5\end{array}$ & $\begin{array}{r}12309 \\
2\end{array}$ & 37.7 & $\begin{array}{r}14.86 \\
53\end{array}$ & 2.1 & 1.2556 & 2.8 & $\begin{array}{r}0.135 \\
4\end{array}$ & $\begin{array}{r}1 . \\
8\end{array}$ & $\begin{array}{r}0.6 \\
7\end{array}$ & 818.4 & 14.1 & 825.9 & 15.6 & 846.1 & 42.9 & 818.4 & 14.1 & 96.7 \\
\hline $\begin{array}{l}\text { BU0722- } \\
27\end{array}$ & 256 & 39492 & 1.2 & $\begin{array}{r}14.68 \\
32 \\
\end{array}$ & 1.5 & 1.2885 & 1.8 & $\begin{array}{r}0.137 \\
2 \\
\end{array}$ & $\begin{array}{r}1 . \\
0\end{array}$ & $\begin{array}{r}0.5 \\
6 \\
\end{array}$ & 828.9 & 7.8 & 840.6 & 10.2 & 871.7 & 30.5 & 828.9 & 7.8 & 95.1 \\
\hline $\begin{array}{l}\text { BU0722- } \\
79\end{array}$ & $\begin{array}{r}116 \\
4 \\
\end{array}$ & $\begin{array}{r}12291 \\
6 \\
\end{array}$ & 4.8 & $\begin{array}{r}13.93 \\
16 \\
\end{array}$ & 1.2 & 1.4008 & 4.1 & $\begin{array}{r}0.141 \\
5 \\
\end{array}$ & $\begin{array}{r}4 . \\
0 \\
\end{array}$ & $\begin{array}{r}0.9 \\
6 \\
\end{array}$ & 853.3 & 31.7 & 889.3 & 24.5 & 979.7 & 24.2 & 853.3 & 31.7 & 87.1 \\
\hline $\begin{array}{l}\text { BU0722- } \\
51\end{array}$ & 840 & $\begin{array}{r}16263 \\
2 \\
\end{array}$ & 16.4 & $\begin{array}{r}14.01 \\
64 \\
\end{array}$ & 2.7 & 1.4456 & 2.8 & $\begin{array}{r}0.147 \\
0 \\
\end{array}$ & $\begin{array}{r}1 . \\
0\end{array}$ & $\begin{array}{r}0.3 \\
5 \\
\end{array}$ & 883.9 & 8.3 & 908.1 & 17.0 & 967.3 & 54.1 & 883.9 & 8.3 & 91.4 \\
\hline $\begin{array}{l}\text { BU0722- } \\
20\end{array}$ & 363 & 67088 & 7.3 & $\begin{array}{r}14.05 \\
99\end{array}$ & 2.6 & 1.4619 & 4.6 & $\begin{array}{r}0.149 \\
1\end{array}$ & $\begin{array}{r}3 . \\
8\end{array}$ & $\begin{array}{r}0.8 \\
3\end{array}$ & 895.8 & 32.1 & 914.8 & 28.0 & 961.0 & 53.5 & 895.8 & 32.1 & 93.2 \\
\hline $\begin{array}{l}\text { BU0722- } \\
72\end{array}$ & 729 & $\begin{array}{r}10097 \\
6 \\
\end{array}$ & 4.1 & $\begin{array}{r}13.82 \\
92 \\
\end{array}$ & 2.0 & 1.5169 & 2.2 & $\begin{array}{r}0.152 \\
1 \\
\end{array}$ & $\begin{array}{r}1 . \\
0 \\
\end{array}$ & $\begin{array}{r}0.4 \\
5\end{array}$ & 913.0 & 8.5 & 937.2 & 13.5 & 994.7 & 40.0 & 913.0 & 8.5 & 91.8 \\
\hline $\begin{array}{l}\text { BU0722- } \\
36\end{array}$ & 932 & $\begin{array}{r}29647 \\
2\end{array}$ & 9.5 & $\begin{array}{r}14.30 \\
58\end{array}$ & 1.2 & 1.4677 & 1.6 & $\begin{array}{r}0.152 \\
3\end{array}$ & $\begin{array}{r}1 . \\
0\end{array}$ & $\begin{array}{r}0.6 \\
3\end{array}$ & 913.8 & 8.5 & 917.2 & 9.6 & 925.5 & 25.3 & 913.8 & 8.5 & 98.7 \\
\hline $\begin{array}{l}\text { BU0722- } \\
77\end{array}$ & $\begin{array}{r}119 \\
4 \\
\end{array}$ & $\begin{array}{r}14780 \\
4 \\
\end{array}$ & 8.5 & $\begin{array}{r}14.34 \\
01 \\
\end{array}$ & 1.3 & 1.4694 & 1.6 & $\begin{array}{r}0.152 \\
8 \\
\end{array}$ & $\begin{array}{r}1 . \\
0\end{array}$ & $\begin{array}{r}0.6 \\
2 \\
\end{array}$ & 916.8 & 8.5 & 917.9 & 9.8 & 920.5 & 26.1 & 916.8 & 8.5 & 99.6 \\
\hline $\begin{array}{l}\text { BU0722- } \\
92\end{array}$ & 265 & 84408 & 1.8 & $\begin{array}{r}13.95 \\
02 \\
\end{array}$ & 1.8 & 1.5370 & 2.1 & $\begin{array}{r}0.155 \\
5 \\
\end{array}$ & $\begin{array}{l}1 . \\
0\end{array}$ & $\begin{array}{r}0.4 \\
9 \\
\end{array}$ & 931.7 & 8.7 & 945.3 & 12.6 & 977.0 & 36.5 & 931.7 & 8.7 & 95.4 \\
\hline BU0722- & 291 & 58744 & 1.4 & 14.17 & 1.1 & 1.5325 & 1.5 & 0.157 & 1. & 0.6 & 943.2 & 8.8 & 943.5 & 9.1 & 944.1 & 22.5 & 943.2 & 8.8 & 99.9 \\
\hline
\end{tabular}




\begin{tabular}{|c|c|c|c|c|c|c|c|c|c|c|c|c|c|c|c|c|c|c|c|}
\hline 46 & & & & 62 & & & & 6 & 0 & 7 & & & & & & & & & \\
\hline $\begin{array}{l}\text { BU0722- } \\
107\end{array}$ & 380 & $\begin{array}{r}18697 \\
36 \\
\end{array}$ & 5.7 & $\begin{array}{r}14.00 \\
46 \\
\end{array}$ & 1.0 & 1.5942 & 1.4 & $\begin{array}{r}0.161 \\
9 \\
\end{array}$ & $\begin{array}{r}1 . \\
0\end{array}$ & $\begin{array}{r}0.7 \\
1 \\
\end{array}$ & 967.4 & 9.0 & 967.9 & 8.8 & 969.0 & 20.4 & 967.4 & 9.0 & 99.8 \\
\hline $\begin{array}{l}\text { BU0722- } \\
41\end{array}$ & 534 & $\begin{array}{r}14117 \\
2\end{array}$ & 10.0 & $\begin{array}{r}13.94 \\
64\end{array}$ & 1.9 & 1.6442 & 2.5 & $\begin{array}{r}0.166 \\
3\end{array}$ & $\begin{array}{r}1 . \\
5\end{array}$ & $\begin{array}{r}0.6 \\
2\end{array}$ & 991.7 & 14.1 & 987.3 & 15.6 & 977.5 & 39.5 & 977.5 & 39.5 & $\begin{array}{r}101 . \\
5\end{array}$ \\
\hline $\begin{array}{l}\text { BU0722- } \\
96\end{array}$ & 346 & 75868 & 5.8 & $\begin{array}{r}13.71 \\
22\end{array}$ & 2.6 & 1.5871 & 2.9 & $\begin{array}{r}0.157 \\
8\end{array}$ & $\begin{array}{r}1 . \\
2\end{array}$ & $\begin{array}{r}0.4 \\
2\end{array}$ & 944.8 & 10.7 & 965.2 & 17.9 & $\begin{array}{r}1011 . \\
9\end{array}$ & 52.7 & 1011.9 & 52.7 & 93.4 \\
\hline $\begin{array}{l}\text { BU0722- } \\
34\end{array}$ & 366 & 52020 & 2.8 & $\begin{array}{r}13.68 \\
64 \\
\end{array}$ & 2.3 & 1.5602 & 2.9 & $\begin{array}{r}0.154 \\
9 \\
\end{array}$ & $\begin{array}{l}1 . \\
7 \\
\end{array}$ & $\begin{array}{r}0.5 \\
9\end{array}$ & 928.2 & 14.8 & 954.6 & 17.9 & $\begin{array}{r}1015 . \\
7\end{array}$ & 47.4 & 1015.7 & 47.4 & 91.4 \\
\hline $\begin{array}{l}\text { BU0722- } \\
25\end{array}$ & 98 & 15100 & 1.8 & $\begin{array}{r}13.63 \\
90 \\
\end{array}$ & 1.8 & 1.6049 & 2.3 & $\begin{array}{r}0.158 \\
8 \\
\end{array}$ & $\begin{array}{r}1 . \\
5\end{array}$ & $\begin{array}{r}0.6 \\
2 \\
\end{array}$ & 949.8 & 12.9 & 972.1 & 14.7 & $\begin{array}{r}1022 . \\
8 \\
\end{array}$ & 37.1 & 1022.8 & 37.1 & 92.9 \\
\hline $\begin{array}{l}\text { BU0722- } \\
62\end{array}$ & 785 & 73388 & 1.2 & $\begin{array}{r}13.38 \\
13\end{array}$ & 1.6 & 1.8148 & 2.9 & $\begin{array}{r}0.176 \\
1\end{array}$ & $\begin{array}{r}2 . \\
4\end{array}$ & $\begin{array}{r}0.8 \\
3 \\
\end{array}$ & $\begin{array}{r}1045 . \\
8 \\
\end{array}$ & 23.0 & 1050.8 & 18.8 & $\begin{array}{r}1061 . \\
3 \\
\end{array}$ & 32.2 & 1061.3 & 32.2 & 98.5 \\
\hline $\begin{array}{l}\text { BU0722- } \\
11\end{array}$ & 667 & $\begin{array}{r}12824 \\
4\end{array}$ & 1.9 & $\begin{array}{r}13.31 \\
97\end{array}$ & 3.0 & 1.9662 & 3.2 & $\begin{array}{r}0.189 \\
9\end{array}$ & $\begin{array}{l}1 . \\
0\end{array}$ & $\begin{array}{r}0.3 \\
2\end{array}$ & $\begin{array}{r}1121 . \\
1\end{array}$ & 10.4 & 1104.0 & 21.3 & $\begin{array}{r}1070 . \\
5\end{array}$ & 60.3 & 1070.5 & 60.3 & $\begin{array}{r}104 . \\
7\end{array}$ \\
\hline $\begin{array}{l}\text { BU0722- } \\
17\end{array}$ & 433 & 71096 & 2.4 & $\begin{array}{r}13.29 \\
42\end{array}$ & 1.2 & 1.5849 & 2.5 & $\begin{array}{r}0.152 \\
8\end{array}$ & $\begin{array}{r}2 . \\
2\end{array}$ & $\begin{array}{r}0.8 \\
7\end{array}$ & 916.7 & 18.5 & 964.3 & 15.5 & $\begin{array}{r}1074 . \\
4\end{array}$ & 24.7 & 1074.4 & 24.7 & 85.3 \\
\hline $\begin{array}{l}\text { BU0722- } \\
59\end{array}$ & 336 & 41624 & 1.3 & $\begin{array}{r}13.08 \\
88\end{array}$ & 1.1 & 1.6928 & 2.2 & $\begin{array}{r}0.160 \\
7\end{array}$ & $\begin{array}{r}2 . \\
0\end{array}$ & $\begin{array}{r}0.8 \\
7 \\
\end{array}$ & 960.6 & 17.4 & 1005.8 & 14.3 & $\begin{array}{r}1105 . \\
6\end{array}$ & 22.0 & 1105.6 & 22.0 & 86.9 \\
\hline $\begin{array}{l}\text { BU0722- } \\
53\end{array}$ & 521 & $\begin{array}{r}12490 \\
4 \\
\end{array}$ & 4.7 & $\begin{array}{r}13.02 \\
68 \\
\end{array}$ & 1.7 & 1.8399 & 2.8 & $\begin{array}{r}0.173 \\
8 \\
\end{array}$ & $\begin{array}{r}2 . \\
3\end{array}$ & $\begin{array}{r}0.8 \\
0 \\
\end{array}$ & $\begin{array}{r}1033 . \\
2\end{array}$ & 21.5 & 1059.8 & 18.4 & $\begin{array}{r}1115 . \\
1\end{array}$ & 33.3 & 1115.1 & 33.3 & 92.7 \\
\hline $\begin{array}{l}\text { BU0722- } \\
12\end{array}$ & 370 & 48620 & 1.1 & $\begin{array}{r}12.90 \\
75\end{array}$ & 2.0 & 1.9864 & 3.1 & $\begin{array}{r}0.186 \\
0\end{array}$ & $\begin{array}{r}2 . \\
3\end{array}$ & $\begin{array}{r}0.7 \\
6\end{array}$ & $\begin{array}{r}1099 . \\
4\end{array}$ & 23.6 & 1110.9 & 20.8 & $\begin{array}{r}1133 . \\
4\end{array}$ & 40.0 & 1133.4 & 40.0 & 97.0 \\
\hline $\begin{array}{l}\text { BU0722- } \\
57\end{array}$ & $\begin{array}{r}153 \\
1\end{array}$ & $\begin{array}{r}33430 \\
4\end{array}$ & 8.6 & $\begin{array}{r}12.85 \\
43\end{array}$ & 1.0 & 1.9563 & 1.6 & $\begin{array}{r}0.182 \\
4\end{array}$ & $\begin{array}{r}1 . \\
3\end{array}$ & $\begin{array}{r}0.7 \\
9\end{array}$ & $\begin{array}{r}1080 . \\
0\end{array}$ & 13.0 & 1100.6 & 11.1 & $\begin{array}{r}1141 . \\
6\end{array}$ & 19.9 & 1141.6 & 19.9 & 94.6 \\
\hline $\begin{array}{l}\text { BU0722- } \\
30\end{array}$ & 272 & 61068 & 2.0 & $\begin{array}{r}12.84 \\
73\end{array}$ & 1.0 & 2.0390 & 3.1 & $\begin{array}{r}0.190 \\
0\end{array}$ & $\begin{array}{r}2 . \\
9\end{array}$ & $\begin{array}{r}0.9 \\
5\end{array}$ & $\begin{array}{r}1121 . \\
3\end{array}$ & 30.2 & 1128.6 & 21.1 & $\begin{array}{r}1142 . \\
7\end{array}$ & 19.9 & 1142.7 & 19.9 & 98.1 \\
\hline $\begin{array}{l}\text { BU0722- } \\
19\end{array}$ & 651 & $\begin{array}{r}14827 \\
6 \\
\end{array}$ & 2.7 & $\begin{array}{r}12.80 \\
89 \\
\end{array}$ & 1.8 & 2.0259 & 3.1 & $\begin{array}{r}0.188 \\
2 \\
\end{array}$ & $\begin{array}{r}2 . \\
6\end{array}$ & $\begin{array}{r}0.8 \\
2 \\
\end{array}$ & $\begin{array}{r}1111 . \\
6\end{array}$ & 26.2 & 1124.2 & 21.3 & $\begin{array}{r}1148 . \\
7\end{array}$ & 35.4 & 1148.7 & 35.4 & 96.8 \\
\hline $\begin{array}{l}\text { BU0722- } \\
86\end{array}$ & 553 & $\begin{array}{r}13054 \\
4\end{array}$ & 3.2 & $\begin{array}{r}12.72 \\
39\end{array}$ & 1.0 & 2.0634 & 1.6 & $\begin{array}{r}0.190 \\
4\end{array}$ & $\begin{array}{r}1 . \\
2\end{array}$ & $\begin{array}{r}0.7 \\
7\end{array}$ & $\begin{array}{r}1123 . \\
6\end{array}$ & 12.3 & 1136.7 & 10.6 & $\begin{array}{r}1161 . \\
9\end{array}$ & 19.8 & 1161.9 & 19.8 & 96.7 \\
\hline $\begin{array}{l}\text { BU0722- } \\
78\end{array}$ & 412 & 81272 & 2.4 & $\begin{array}{r}12.66 \\
50\end{array}$ & 1.6 & 1.9186 & 2.3 & $\begin{array}{r}0.176 \\
2\end{array}$ & $\begin{array}{l}1 . \\
7\end{array}$ & $\begin{array}{r}0.7 \\
3\end{array}$ & $\begin{array}{r}1046 . \\
4\end{array}$ & 16.2 & 1087.6 & 15.3 & $\begin{array}{r}1171 . \\
1\end{array}$ & 30.7 & 1171.1 & 30.7 & 89.4 \\
\hline $\begin{array}{l}\text { BU0722- } \\
105\end{array}$ & 201 & 71840 & 1.9 & $\begin{array}{r}12.65 \\
95\end{array}$ & 1.5 & 2.1292 & 2.1 & $\begin{array}{r}0.195 \\
5\end{array}$ & $\begin{array}{l}1 . \\
5\end{array}$ & $\begin{array}{r}0.6 \\
9\end{array}$ & $\begin{array}{r}1151 . \\
1\end{array}$ & 15.3 & 1158.3 & 14.4 & $\begin{array}{r}1171 . \\
9\end{array}$ & 29.7 & 1171.9 & 29.7 & 98.2 \\
\hline BU0722-1 & 206 & $\begin{array}{r}25135 \\
2\end{array}$ & 3.5 & $\begin{array}{r}12.13 \\
83\end{array}$ & 3.6 & 2.3674 & 6.8 & $\begin{array}{r}0.208 \\
4\end{array}$ & $\begin{array}{r}5 . \\
8\end{array}$ & $\begin{array}{r}0.8 \\
5\end{array}$ & $\begin{array}{r}1220 . \\
4\end{array}$ & 64.2 & 1232.8 & 48.6 & $\begin{array}{r}1254 . \\
6\end{array}$ & 70.6 & 1254.6 & 70.6 & 97.3 \\
\hline $\begin{array}{l}\text { BU0722- } \\
13\end{array}$ & 339 & 54208 & 1.2 & $\begin{array}{r}12.07 \\
82 \\
\end{array}$ & 2.1 & 2.3393 & 3.2 & $\begin{array}{r}0.204 \\
9 \\
\end{array}$ & $\begin{array}{r}2 . \\
5\end{array}$ & $\begin{array}{r}0.7 \\
6 \\
\end{array}$ & $\begin{array}{r}1201 . \\
7\end{array}$ & 27.0 & 1224.3 & 22.9 & $\begin{array}{r}1264 . \\
3\end{array}$ & 40.6 & 1264.3 & 40.6 & 95.0 \\
\hline $\begin{array}{l}\text { BU0722- } \\
67\end{array}$ & 863 & $\begin{array}{r}15212 \\
4 \\
\end{array}$ & 1.5 & $\begin{array}{r}11.65 \\
50\end{array}$ & 1.8 & 2.4211 & 3.5 & $\begin{array}{r}0.204 \\
7\end{array}$ & $\begin{array}{r}2 . \\
9\end{array}$ & $\begin{array}{r}0.8 \\
5\end{array}$ & $\begin{array}{r}1200 . \\
3\end{array}$ & 32.2 & 1248.9 & 24.9 & $\begin{array}{r}1333 . \\
7\end{array}$ & 35.2 & 1333.7 & 35.2 & 90.0 \\
\hline $\begin{array}{l}\text { BU0722- } \\
83\end{array}$ & $\begin{array}{r}106 \\
1\end{array}$ & 93280 & 2.0 & $\begin{array}{r}11.06 \\
80\end{array}$ & 1.6 & 2.3784 & 2.3 & $\begin{array}{r}0.190 \\
9\end{array}$ & $\begin{array}{l}1 . \\
7\end{array}$ & $\begin{array}{r}0.7 \\
2\end{array}$ & $\begin{array}{r}1126 . \\
4\end{array}$ & 17.3 & 1236.1 & 16.5 & $\begin{array}{r}1432 . \\
9\end{array}$ & 30.5 & 1432.9 & 30.5 & 78.6 \\
\hline BU0722-5 & 482 & $\begin{array}{r}13537 \\
2\end{array}$ & 5.1 & $\begin{array}{r}10.90 \\
74 \\
\end{array}$ & 2.5 & 2.3353 & 2.7 & $\begin{array}{r}0.184 \\
7 \\
\end{array}$ & $\begin{array}{l}1 . \\
0\end{array}$ & $\begin{array}{r}0.3 \\
7 \\
\end{array}$ & $\begin{array}{r}1092 . \\
8 \\
\end{array}$ & 10.1 & 1223.1 & 19.3 & $\begin{array}{r}1460 . \\
8 \\
\end{array}$ & 48.1 & 1460.8 & 48.1 & 74.8 \\
\hline $\begin{array}{l}\text { BU0722- } \\
106\end{array}$ & 402 & $\begin{array}{r}15208 \\
8\end{array}$ & 2.0 & $\begin{array}{r}10.37 \\
28\end{array}$ & 1.8 & 3.5985 & 2.1 & $\begin{array}{r}0.270 \\
7\end{array}$ & $\begin{array}{r}1 . \\
1\end{array}$ & $\begin{array}{r}0.5 \\
4\end{array}$ & $\begin{array}{r}1544 . \\
4\end{array}$ & 15.7 & 1549.2 & 16.7 & $\begin{array}{r}1555 . \\
7\end{array}$ & 33.0 & 1555.7 & 33.0 & 99.3 \\
\hline $\begin{array}{l}\text { BU0722- } \\
109\end{array}$ & 137 & 31520 & 1.6 & $\begin{array}{r}10.35 \\
41\end{array}$ & 1.2 & 3.1837 & 3.5 & $\begin{array}{r}0.239 \\
1\end{array}$ & $\begin{array}{r}3 . \\
3\end{array}$ & $\begin{array}{r}0.9 \\
4\end{array}$ & $\begin{array}{r}1381 . \\
9\end{array}$ & 41.4 & 1453.2 & 27.4 & $\begin{array}{r}1559 . \\
1\end{array}$ & 22.5 & 1559.1 & 22.5 & 88.6 \\
\hline
\end{tabular}




\begin{tabular}{|c|c|c|c|c|c|c|c|c|c|c|c|c|c|c|c|c|c|c|c|}
\hline $\begin{array}{l}\text { BU0722- } \\
101\end{array}$ & $\begin{array}{r}131 \\
3 \\
\end{array}$ & $\begin{array}{r}26066 \\
8 \\
\end{array}$ & 58.5 & $\begin{array}{r}10.33 \\
86 \\
\end{array}$ & 3.9 & 2.7112 & 5.7 & $\begin{array}{r}0.203 \\
3 \\
\end{array}$ & $\begin{array}{r}4 . \\
1\end{array}$ & $\begin{array}{r}0.7 \\
3 \\
\end{array}$ & $\begin{array}{r}1193 . \\
0 \\
\end{array}$ & 44.8 & 1331.5 & 42.0 & $\begin{array}{r}1561 . \\
9 \\
\end{array}$ & 73.0 & 1561.9 & 73.0 & 76.4 \\
\hline $\begin{array}{l}\text { BU0722- } \\
21\end{array}$ & 319 & 80992 & 1.8 & $\begin{array}{r}10.27 \\
28\end{array}$ & 2.1 & 3.0569 & 6.1 & $\begin{array}{r}0.227 \\
8\end{array}$ & $\begin{array}{r}5 . \\
7\end{array}$ & $\begin{array}{r}0.9 \\
4\end{array}$ & $\begin{array}{r}1322 . \\
7\end{array}$ & 68.2 & 1422.0 & 46.5 & $\begin{array}{r}1573 . \\
8\end{array}$ & 38.9 & 1573.8 & 38.9 & 84.0 \\
\hline BU0722-9 & 151 & 56100 & 3.0 & $\begin{array}{r}10.12 \\
12\end{array}$ & 1.4 & 4.0592 & 1.7 & $\begin{array}{r}0.298 \\
0\end{array}$ & $\begin{array}{r}1 . \\
0\end{array}$ & $\begin{array}{r}0.6 \\
0\end{array}$ & $\begin{array}{r}1681 . \\
2\end{array}$ & 15.1 & 1646.1 & 13.8 & $\begin{array}{r}1601 . \\
6\end{array}$ & 25.2 & 1601.6 & 25.2 & $\begin{array}{r}105 . \\
0\end{array}$ \\
\hline $\begin{array}{l}\text { BU0722- } \\
18\end{array}$ & 888 & $\begin{array}{r}38573 \\
2 \\
\end{array}$ & 6.4 & $\begin{array}{r}10.10 \\
51 \\
\end{array}$ & 1.0 & 3.9306 & 1.4 & $\begin{array}{r}0.288 \\
1 \\
\end{array}$ & $\begin{array}{r}1 . \\
0\end{array}$ & $\begin{array}{r}0.7 \\
0 \\
\end{array}$ & $\begin{array}{r}1631 . \\
9\end{array}$ & 14.4 & 1620.0 & 11.5 & $\begin{array}{r}1604 . \\
6\end{array}$ & 18.8 & 1604.6 & 18.8 & $\begin{array}{r}101 . \\
7\end{array}$ \\
\hline $\begin{array}{l}\text { BU0722- } \\
91\end{array}$ & 165 & 27780 & 2.1 & $\begin{array}{r}10.08 \\
17 \\
\end{array}$ & 1.7 & 3.5611 & 2.0 & $\begin{array}{r}0.260 \\
4 \\
\end{array}$ & $\begin{array}{r}1 . \\
1\end{array}$ & $\begin{array}{r}0.5 \\
4 \\
\end{array}$ & $\begin{array}{r}1491 . \\
8 \\
\end{array}$ & 14.1 & 1540.9 & 15.7 & $\begin{array}{r}1608 . \\
9 \\
\end{array}$ & 31.1 & 1608.9 & 31.1 & 92.7 \\
\hline $\begin{array}{l}\text { BU0722- } \\
50\end{array}$ & 377 & 47248 & 1.6 & $\begin{array}{r}10.01 \\
09 \\
\end{array}$ & 1.3 & 3.2242 & 3.8 & $\begin{array}{r}0.234 \\
1 \\
\end{array}$ & $\begin{array}{r}3 . \\
6\end{array}$ & $\begin{array}{r}0.9 \\
4 \\
\end{array}$ & $\begin{array}{r}1355 . \\
9 \\
\end{array}$ & 44.0 & 1463.0 & 29.8 & $\begin{array}{r}1622 . \\
0\end{array}$ & 24.9 & 1622.0 & 24.9 & 83.6 \\
\hline $\begin{array}{l}\text { BU0722- } \\
94\end{array}$ & 329 & 31376 & 3.6 & $\begin{array}{r}9.924 \\
0\end{array}$ & 2.6 & 2.8158 & 2.9 & $\begin{array}{r}0.202 \\
7\end{array}$ & $\begin{array}{r}1 . \\
3\end{array}$ & $\begin{array}{r}0.4 \\
5\end{array}$ & $\begin{array}{r}1189 . \\
6\end{array}$ & 14.0 & 1359.7 & 21.7 & $\begin{array}{r}1638 . \\
2\end{array}$ & 48.0 & 1638.2 & 48.0 & 72.6 \\
\hline $\begin{array}{l}\text { BU0722- } \\
47\end{array}$ & 449 & 92388 & 1.9 & $\begin{array}{r}9.661 \\
2 \\
\end{array}$ & 2.1 & 4.0117 & 2.3 & $\begin{array}{r}0.281 \\
1 \\
\end{array}$ & $\begin{array}{r}1 . \\
0\end{array}$ & $\begin{array}{r}0.4 \\
3 \\
\end{array}$ & $\begin{array}{r}1596 . \\
9 \\
\end{array}$ & 14.1 & 1636.6 & 18.9 & $\begin{array}{r}1687 . \\
9 \\
\end{array}$ & 38.8 & 1687.9 & 38.8 & 94.6 \\
\hline $\begin{array}{l}\text { BU0722- } \\
44\end{array}$ & 316 & 84624 & 1.6 & $\begin{array}{r}9.644 \\
3 \\
\end{array}$ & 1.7 & 3.8491 & 2.5 & $\begin{array}{r}0.269 \\
2 \\
\end{array}$ & $\begin{array}{r}1 . \\
8\end{array}$ & $\begin{array}{r}0.7 \\
4\end{array}$ & $\begin{array}{r}1536 . \\
9\end{array}$ & 25.0 & 1603.1 & 19.9 & $\begin{array}{r}1691 . \\
2\end{array}$ & 30.4 & 1691.2 & 30.4 & 90.9 \\
\hline $\begin{array}{l}\text { BU0722- } \\
37\end{array}$ & 980 & $\begin{array}{r}35879 \\
6 \\
\end{array}$ & 7.6 & $\begin{array}{r}9.609 \\
0 \\
\end{array}$ & 1.4 & 4.1110 & 1.9 & $\begin{array}{r}0.286 \\
5 \\
\end{array}$ & $\begin{array}{r}1 . \\
4\end{array}$ & $\begin{array}{r}0.7 \\
0 \\
\end{array}$ & $\begin{array}{r}1624 . \\
0 \\
\end{array}$ & 19.5 & 1656.5 & 15.8 & $\begin{array}{r}1697 . \\
9 \\
\end{array}$ & 25.2 & 1697.9 & 25.2 & 95.6 \\
\hline $\begin{array}{l}\text { BU0722- } \\
38\end{array}$ & 286 & $\begin{array}{r}11888 \\
4\end{array}$ & 1.2 & $\begin{array}{r}9.604 \\
5\end{array}$ & 1.1 & 4.2444 & 2.1 & $\begin{array}{r}0.295 \\
7\end{array}$ & $\begin{array}{r}1 . \\
8\end{array}$ & $\begin{array}{r}0.8 \\
6\end{array}$ & $\begin{array}{r}1669 . \\
7\end{array}$ & 26.8 & 1682.7 & 17.4 & $\begin{array}{r}1698 . \\
8\end{array}$ & 20.1 & 1698.8 & 20.1 & 98.3 \\
\hline $\begin{array}{l}\text { BU0722- } \\
31\end{array}$ & 319 & $\begin{array}{r}12652 \\
4\end{array}$ & 3.2 & $\begin{array}{r}9.571 \\
0\end{array}$ & 1.0 & 4.3475 & 1.5 & $\begin{array}{r}0.301 \\
8\end{array}$ & $\begin{array}{r}1 . \\
1\end{array}$ & $\begin{array}{r}0.7 \\
3\end{array}$ & $\begin{array}{r}1700 . \\
1\end{array}$ & 16.0 & 1702.4 & 12.1 & $\begin{array}{r}1705 . \\
2\end{array}$ & 18.4 & 1705.2 & 18.4 & 99.7 \\
\hline $\begin{array}{l}\text { BU0722- } \\
98\end{array}$ & 356 & $\begin{array}{r}11811 \\
6 \\
\end{array}$ & 4.7 & $\begin{array}{r}9.565 \\
1 \\
\end{array}$ & 1.1 & 4.0024 & 1.9 & $\begin{array}{r}0.277 \\
7 \\
\end{array}$ & $\begin{array}{r}1 . \\
6\end{array}$ & $\begin{array}{r}0.8 \\
4 \\
\end{array}$ & $\begin{array}{r}1579 . \\
6 \\
\end{array}$ & 23.0 & 1634.7 & 15.8 & $\begin{array}{r}1706 . \\
3\end{array}$ & 19.3 & 1706.3 & 19.3 & 92.6 \\
\hline $\begin{array}{l}\text { BU0722- } \\
76\end{array}$ & 852 & $\begin{array}{r}16006 \\
4 \\
\end{array}$ & 2.0 & $\begin{array}{r}9.543 \\
9 \\
\end{array}$ & 1.1 & 3.8233 & 5.7 & $\begin{array}{r}0.264 \\
6 \\
\end{array}$ & $\begin{array}{r}5 . \\
6 \\
\end{array}$ & $\begin{array}{r}0.9 \\
8 \\
\end{array}$ & $\begin{array}{r}1513 . \\
6 \\
\end{array}$ & 75.3 & 1597.7 & 45.8 & $\begin{array}{r}1710 . \\
4\end{array}$ & 20.6 & 1710.4 & 20.6 & 88.5 \\
\hline $\begin{array}{l}\text { BU0722- } \\
16\end{array}$ & 202 & 91956 & 4.3 & $\begin{array}{r}9.524 \\
9 \\
\end{array}$ & 1.0 & 4.2446 & 1.4 & $\begin{array}{r}0.293 \\
2 \\
\end{array}$ & $\begin{array}{r}1 . \\
0\end{array}$ & $\begin{array}{r}0.7 \\
0 \\
\end{array}$ & $\begin{array}{r}1657 . \\
6 \\
\end{array}$ & 14.6 & 1682.7 & 11.8 & $\begin{array}{r}1714 . \\
1 \\
\end{array}$ & 18.9 & 1714.1 & 18.9 & 96.7 \\
\hline $\begin{array}{l}\text { BU0722- } \\
71\end{array}$ & 139 & 43568 & 1.0 & $\begin{array}{r}9.476 \\
4\end{array}$ & 1.8 & 4.3295 & 2.0 & $\begin{array}{r}0.297 \\
6\end{array}$ & $\begin{array}{r}1 . \\
0\end{array}$ & $\begin{array}{r}0.4 \\
9\end{array}$ & $\begin{array}{r}1679 . \\
2\end{array}$ & 14.8 & 1699.0 & 16.8 & $\begin{array}{r}1723 . \\
5\end{array}$ & 32.7 & 1723.5 & 32.7 & 97.4 \\
\hline $\begin{array}{l}\text { BU0722- } \\
56\end{array}$ & 249 & $\begin{array}{r}12454 \\
4 \\
\end{array}$ & 2.4 & $\begin{array}{r}9.467 \\
0 \\
\end{array}$ & 1.0 & 4.4148 & 1.5 & $\begin{array}{r}0.303 \\
1 \\
\end{array}$ & $\begin{array}{r}1 . \\
2\end{array}$ & $\begin{array}{r}0.7 \\
6 \\
\end{array}$ & $\begin{array}{r}1706 . \\
8 \\
\end{array}$ & 17.5 & 1715.1 & 12.7 & $\begin{array}{r}1725 . \\
3 \\
\end{array}$ & 18.4 & 1725.3 & 18.4 & 98.9 \\
\hline $\begin{array}{l}\text { BU0722- } \\
45\end{array}$ & 342 & $\begin{array}{r}12282 \\
4 \\
\end{array}$ & 1.6 & $\begin{array}{r}9.462 \\
8 \\
\end{array}$ & 1.8 & 4.3167 & 2.1 & $\begin{array}{r}0.296 \\
3 \\
\end{array}$ & $\begin{array}{r}1 . \\
1\end{array}$ & $\begin{array}{r}0.5 \\
2 \\
\end{array}$ & $\begin{array}{r}1672 . \\
7 \\
\end{array}$ & 15.9 & 1696.6 & 17.0 & $\begin{array}{r}1726 . \\
1 \\
\end{array}$ & 32.3 & 1726.1 & 32.3 & 96.9 \\
\hline $\begin{array}{l}\text { BU0722- } \\
68\end{array}$ & $\begin{array}{r}110 \\
0 \\
\end{array}$ & $\begin{array}{r}14368 \\
4 \\
\end{array}$ & 5.3 & $\begin{array}{r}9.444 \\
5 \\
\end{array}$ & 1.3 & 4.1534 & 2.6 & $\begin{array}{r}0.284 \\
5 \\
\end{array}$ & $\begin{array}{r}2 . \\
3 \\
\end{array}$ & $\begin{array}{r}0.8 \\
8 \\
\end{array}$ & $\begin{array}{r}1614 . \\
0 \\
\end{array}$ & 32.8 & 1664.9 & 21.5 & $\begin{array}{r}1729 . \\
7 \\
\end{array}$ & 23.1 & 1729.7 & 23.1 & 93.3 \\
\hline $\begin{array}{l}\text { BU0722- } \\
93\end{array}$ & 496 & $\begin{array}{r}14584 \\
0\end{array}$ & 1.9 & $\begin{array}{r}9.436 \\
5\end{array}$ & 1.1 & 4.3086 & 1.5 & $\begin{array}{r}0.294 \\
9\end{array}$ & $\begin{array}{r}1 . \\
0\end{array}$ & $\begin{array}{r}0.6 \\
6\end{array}$ & $\begin{array}{r}1665 . \\
9\end{array}$ & 14.7 & 1695.0 & 12.4 & $\begin{array}{r}1731 . \\
2\end{array}$ & 20.7 & 1731.2 & 20.7 & 96.2 \\
\hline $\begin{array}{l}\text { BU0722- } \\
104\end{array}$ & 140 & 53592 & 2.3 & $\begin{array}{r}9.404 \\
4 \\
\end{array}$ & 1.4 & 4.0785 & 1.7 & $\begin{array}{r}0.278 \\
2 \\
\end{array}$ & $\begin{array}{r}1 . \\
0\end{array}$ & $\begin{array}{r}0.5 \\
7\end{array}$ & $\begin{array}{r}1582 . \\
2\end{array}$ & 14.0 & 1650.0 & 14.2 & $\begin{array}{r}1737 . \\
5\end{array}$ & 26.2 & 1737.5 & 26.2 & 91.1 \\
\hline $\begin{array}{l}\text { BU0722- } \\
95\end{array}$ & $\begin{array}{r}101 \\
9\end{array}$ & $\begin{array}{r}36643 \\
2\end{array}$ & 2.0 & $\begin{array}{r}9.395 \\
3\end{array}$ & 1.3 & 4.3026 & 1.8 & $\begin{array}{r}0.293 \\
2\end{array}$ & $\begin{array}{r}1 . \\
3\end{array}$ & $\begin{array}{r}0.7 \\
1\end{array}$ & $\begin{array}{r}1657 . \\
4\end{array}$ & 19.0 & 1693.9 & 15.0 & $\begin{array}{r}1739 . \\
2\end{array}$ & 23.5 & 1739.2 & 23.5 & 95.3 \\
\hline $\begin{array}{l}\text { BU0722- } \\
58\end{array}$ & 732 & $\begin{array}{r}12754 \\
0 \\
\end{array}$ & 2.6 & $\begin{array}{r}9.388 \\
8 \\
\end{array}$ & 1.0 & 3.5730 & 1.8 & $\begin{array}{r}0.243 \\
3 \\
\end{array}$ & $\begin{array}{r}1 . \\
4\end{array}$ & $\begin{array}{r}0.8 \\
2 \\
\end{array}$ & $\begin{array}{r}1403 . \\
8 \\
\end{array}$ & 18.2 & 1543.6 & 13.9 & $\begin{array}{r}1740 . \\
5 \\
\end{array}$ & 18.3 & 1740.5 & 18.3 & 80.7 \\
\hline $\begin{array}{l}\text { BU0722- } \\
64\end{array}$ & 492 & $\begin{array}{r}14110 \\
8 \\
\end{array}$ & 1.2 & $\begin{array}{r}9.364 \\
3 \\
\end{array}$ & 1.3 & 4.5521 & 1.9 & $\begin{array}{r}0.309 \\
2 \\
\end{array}$ & $\begin{array}{r}1 . \\
3 \\
\end{array}$ & $\begin{array}{r}0.7 \\
1 \\
\end{array}$ & $\begin{array}{r}1736 . \\
6 \\
\end{array}$ & 20.2 & 1740.6 & 15.6 & $\begin{array}{r}1745 . \\
3 \\
\end{array}$ & 24.2 & 1745.3 & 24.2 & 99.5 \\
\hline BU0722- & 423 & 12758 & 3.2 & 9.340 & 1.0 & 4.4591 & 1.4 & 0.302 & 1. & 0.7 & 1701. & 15.0 & 1723.4 & 11.7 & 1750. & 18.3 & 1750.0 & 18.3 & 97.2 \\
\hline
\end{tabular}




\begin{tabular}{|c|c|c|c|c|c|c|c|c|c|c|c|c|c|c|c|c|c|c|c|}
\hline 102 & & 4 & & 5 & & & & 1 & 0 & 1 & 6 & & & & 0 & & & & \\
\hline $\begin{array}{l}\text { BU0722- } \\
29\end{array}$ & 501 & $\begin{array}{r}19273 \\
6 \\
\end{array}$ & 1.8 & $\begin{array}{r}9.282 \\
7 \\
\end{array}$ & 1.8 & 4.3723 & 2.2 & $\begin{array}{r}0.294 \\
4 \\
\end{array}$ & $\begin{array}{r}1 . \\
3\end{array}$ & $\begin{array}{r}0.5 \\
7 \\
\end{array}$ & $\begin{array}{r}1663 . \\
3 \\
\end{array}$ & 18.3 & 1707.1 & 18.2 & $\begin{array}{r}1761 . \\
3 \\
\end{array}$ & 33.3 & 1761.3 & 33.3 & 94.4 \\
\hline $\begin{array}{l}\text { BU0722- } \\
100\end{array}$ & 242 & 89540 & 4.9 & $\begin{array}{r}9.261 \\
1\end{array}$ & 2.8 & 4.5063 & 3.0 & $\begin{array}{r}0.302 \\
7\end{array}$ & $\begin{array}{r}1 . \\
0\end{array}$ & $\begin{array}{r}0.3 \\
3\end{array}$ & $\begin{array}{r}1704 . \\
6\end{array}$ & 15.0 & 1732.1 & 24.9 & $\begin{array}{r}1765 . \\
6\end{array}$ & 51.5 & 1765.6 & 51.5 & 96.5 \\
\hline $\begin{array}{l}\text { BU0722- } \\
40\end{array}$ & 410 & 60380 & 2.2 & $\begin{array}{r}8.862 \\
0 \\
\end{array}$ & 4.9 & 4.0078 & 5.2 & $\begin{array}{r}0.257 \\
6 \\
\end{array}$ & $\begin{array}{r}1 . \\
8\end{array}$ & $\begin{array}{r}0.3 \\
4 \\
\end{array}$ & $\begin{array}{r}1477 . \\
5 \\
\end{array}$ & 23.1 & 1635.8 & 42.2 & $\begin{array}{r}1845 . \\
7\end{array}$ & 88.5 & 1845.7 & 88.5 & 80.1 \\
\hline $\begin{array}{l}\text { BU0722- } \\
63\end{array}$ & 282 & $\begin{array}{r}10260 \\
8\end{array}$ & 2.4 & $\begin{array}{r}8.605 \\
4\end{array}$ & 2.0 & 5.5124 & 2.6 & $\begin{array}{r}0.344 \\
0\end{array}$ & $\begin{array}{r}1 . \\
6\end{array}$ & $\begin{array}{r}0.6 \\
3\end{array}$ & $\begin{array}{r}1906 . \\
1\end{array}$ & 26.4 & 1902.5 & 21.9 & $\begin{array}{r}1898 . \\
7\end{array}$ & 35.8 & 1898.7 & 35.8 & $\begin{array}{r}100 . \\
4\end{array}$ \\
\hline $\begin{array}{l}\text { BU0722- } \\
74\end{array}$ & 239 & $\begin{array}{r}10831 \\
2 \\
\end{array}$ & 3.4 & $\begin{array}{r}8.193 \\
5 \\
\end{array}$ & 1.1 & 6.0846 & 2.0 & $\begin{array}{r}0.361 \\
6 \\
\end{array}$ & $\begin{array}{r}1 . \\
7 \\
\end{array}$ & $\begin{array}{r}0.8 \\
4 \\
\end{array}$ & $\begin{array}{r}1989 . \\
7 \\
\end{array}$ & 28.6 & 1988.0 & 17.4 & $\begin{array}{r}1986 . \\
4 \\
\end{array}$ & 19.4 & 1986.4 & 19.4 & $\begin{array}{r}100 . \\
2 \\
\end{array}$ \\
\hline $\begin{array}{l}\text { BU0722- } \\
54\end{array}$ & 429 & $\begin{array}{r}11021 \\
2\end{array}$ & 2.0 & $\begin{array}{r}6.563 \\
9\end{array}$ & 1.3 & 6.6040 & 2.7 & $\begin{array}{r}0.314 \\
4\end{array}$ & $\begin{array}{r}2 . \\
3\end{array}$ & $\begin{array}{r}0.8 \\
7\end{array}$ & $\begin{array}{r}1762 . \\
3\end{array}$ & 35.6 & 2059.9 & 23.5 & $\begin{array}{r}2372 . \\
4\end{array}$ & 22.7 & 2372.4 & 22.7 & 74.3 \\
\hline BU0722-8 & 85 & $\begin{array}{r}10899 \\
2 \\
\end{array}$ & 1.1 & $\begin{array}{r}6.547 \\
3 \\
\end{array}$ & 2.3 & 9.1112 & 4.2 & $\begin{array}{r}0.432 \\
6 \\
\end{array}$ & $\begin{array}{r}3 . \\
5\end{array}$ & $\begin{array}{r}0.8 \\
4\end{array}$ & $\begin{array}{r}2317 . \\
6\end{array}$ & 68.3 & 2349.2 & 38.4 & $\begin{array}{r}2376 . \\
8\end{array}$ & 39.0 & 2376.8 & 39.0 & 97.5 \\
\hline $\begin{array}{l}\text { BU0722- } \\
52\end{array}$ & 598 & $\begin{array}{r}21466 \\
4 \\
\end{array}$ & 2.8 & $\begin{array}{r}6.501 \\
0 \\
\end{array}$ & 1.4 & 7.5232 & 2.8 & $\begin{array}{r}0.354 \\
7 \\
\end{array}$ & $\begin{array}{r}2 . \\
5\end{array}$ & $\begin{array}{r}0.8 \\
6 \\
\end{array}$ & $\begin{array}{r}1957 . \\
1\end{array}$ & 41.4 & 2175.8 & 25.5 & $\begin{array}{r}2388 . \\
8\end{array}$ & 24.5 & 2388.8 & 24.5 & 81.9 \\
\hline $\begin{array}{l}\text { BU0722- } \\
26\end{array}$ & 484 & $\begin{array}{r}12720 \\
4 \\
\end{array}$ & 1.5 & $\begin{array}{r}6.477 \\
7 \\
\end{array}$ & 2.7 & 6.7932 & 2.9 & $\begin{array}{r}0.319 \\
1 \\
\end{array}$ & $\begin{array}{r}1 . \\
0\end{array}$ & $\begin{array}{r}0.3 \\
5 \\
\end{array}$ & $\begin{array}{r}1785 . \\
6 \\
\end{array}$ & 15.6 & 2084.8 & 25.4 & $\begin{array}{r}2395 . \\
0 \\
\end{array}$ & 45.8 & 2395.0 & 45.8 & 74.6 \\
\hline $\begin{array}{l}\text { BU0722- } \\
33\end{array}$ & 306 & $\begin{array}{r}11545 \\
6 \\
\end{array}$ & 1.8 & $\begin{array}{r}6.460 \\
8 \\
\end{array}$ & 3.3 & 7.8850 & 3.7 & $\begin{array}{r}0.369 \\
5 \\
\end{array}$ & $\begin{array}{r}1 . \\
5\end{array}$ & $\begin{array}{r}0.4 \\
2\end{array}$ & $\begin{array}{r}2026 . \\
9\end{array}$ & 26.6 & 2218.0 & 33.1 & $\begin{array}{r}2399 . \\
4\end{array}$ & 56.8 & 2399.4 & 56.8 & 84.5 \\
\hline $\begin{array}{l}\text { BU0722- } \\
87\end{array}$ & 418 & $\begin{array}{r}17591 \\
2\end{array}$ & 3.2 & $\begin{array}{r}6.448 \\
6\end{array}$ & 1.3 & 6.9125 & 3.4 & $\begin{array}{r}0.323 \\
3\end{array}$ & $\begin{array}{r}3 . \\
2\end{array}$ & $\begin{array}{r}0.9 \\
3\end{array}$ & $\begin{array}{r}1805 . \\
8\end{array}$ & 50.6 & 2100.3 & 30.6 & $\begin{array}{r}2402 . \\
6\end{array}$ & 21.4 & 2402.6 & 21.4 & 75.2 \\
\hline $\begin{array}{l}\text { BU0722- } \\
70\end{array}$ & 272 & 88316 & 1.8 & $\begin{array}{r}6.423 \\
9\end{array}$ & 1.7 & 7.1659 & 2.5 & $\begin{array}{r}0.333 \\
9\end{array}$ & $\begin{array}{r}1 . \\
9\end{array}$ & $\begin{array}{r}0.7 \\
3\end{array}$ & $\begin{array}{r}1857 . \\
1\end{array}$ & 30.2 & 2132.3 & 22.7 & $\begin{array}{r}2409 . \\
1\end{array}$ & 29.4 & 2409.1 & 29.4 & 77.1 \\
\hline $\begin{array}{l}\text { BU0722- } \\
90\end{array}$ & 98 & 69208 & 1.3 & $\begin{array}{r}6.345 \\
4 \\
\end{array}$ & 1.4 & 9.5119 & 1.7 & $\begin{array}{r}0.437 \\
8 \\
\end{array}$ & $\begin{array}{r}1 . \\
0 \\
\end{array}$ & $\begin{array}{r}0.5 \\
8 \\
\end{array}$ & $\begin{array}{r}2340 . \\
6 \\
\end{array}$ & 19.6 & 2388.7 & 15.7 & $\begin{array}{r}2430 . \\
0 \\
\end{array}$ & 23.6 & 2430.0 & 23.6 & 96.3 \\
\hline BU0722-6 & 503 & $\begin{array}{r}39656 \\
0 \\
\end{array}$ & 4.0 & $\begin{array}{r}6.232 \\
9 \\
\end{array}$ & 1.2 & 9.4917 & 1.9 & $\begin{array}{r}0.429 \\
1 \\
\end{array}$ & $\begin{array}{r}1 . \\
5\end{array}$ & $\begin{array}{r}0.7 \\
9 \\
\end{array}$ & $\begin{array}{r}2301 . \\
6 \\
\end{array}$ & 29.6 & 2386.7 & 17.8 & $\begin{array}{r}2460 . \\
3 \\
\end{array}$ & 19.9 & 2460.3 & 19.9 & 93.5 \\
\hline $\begin{array}{l}\text { BU0722- } \\
88\end{array}$ & 140 & 10976 & 2.3 & $\begin{array}{r}6.230 \\
5\end{array}$ & 1.0 & 7.9137 & 1.5 & $\begin{array}{r}0.357 \\
6\end{array}$ & $\begin{array}{r}1 . \\
0\end{array}$ & $\begin{array}{r}0.7 \\
0\end{array}$ & $\begin{array}{r}1970 . \\
8\end{array}$ & 17.3 & 2221.2 & 13.1 & $\begin{array}{r}2460 . \\
9\end{array}$ & 17.4 & 2460.9 & 17.4 & 80.1 \\
\hline $\begin{array}{l}\text { BU0722- } \\
39\end{array}$ & 605 & $\begin{array}{r}31876 \\
0 \\
\end{array}$ & 3.2 & $\begin{array}{r}6.052 \\
3 \\
\end{array}$ & 1.9 & $\begin{array}{r}10.368 \\
8 \\
\end{array}$ & 2.1 & $\begin{array}{r}0.455 \\
1 \\
\end{array}$ & $\begin{array}{l}1 . \\
0\end{array}$ & $\begin{array}{r}0.4 \\
8 \\
\end{array}$ & $\begin{array}{r}2418 . \\
1 \\
\end{array}$ & 21.0 & 2468.3 & 19.9 & $\begin{array}{r}2509 . \\
8 \\
\end{array}$ & 31.6 & 2509.8 & 31.6 & 96.3 \\
\hline $\begin{array}{l}\text { BU0742- } \\
34\end{array}$ & $\begin{array}{r}113 \\
1 \\
\end{array}$ & 76725 & 5.4 & $\begin{array}{r}13.03 \\
65 \\
\end{array}$ & 1.4 & 1.4596 & 1.9 & $\begin{array}{r}0.138 \\
0 \\
\end{array}$ & $\begin{array}{r}1 . \\
3\end{array}$ & $\begin{array}{r}0.6 \\
8\end{array}$ & 833.4 & 10.2 & 913.9 & 11.6 & $\begin{array}{r}1113 . \\
6\end{array}$ & 28.4 & 833.4 & 10.2 & 74.8 \\
\hline BU0742-6 & 267 & 35235 & 1.9 & $\begin{array}{r}14.36 \\
10\end{array}$ & 3.5 & 1.4020 & 3.9 & $\begin{array}{r}0.146 \\
0\end{array}$ & $\begin{array}{l}1 . \\
7\end{array}$ & $\begin{array}{r}0.4 \\
3\end{array}$ & 878.6 & 13.6 & 889.8 & 23.1 & 917.5 & 72.6 & 878.6 & 13.6 & 95.8 \\
\hline $\begin{array}{l}\text { BU0742- } \\
75\end{array}$ & 579 & 66800 & 4.0 & $\begin{array}{r}13.60 \\
49 \\
\end{array}$ & 1.7 & 1.6198 & 2.1 & $\begin{array}{r}0.159 \\
8 \\
\end{array}$ & $\begin{array}{r}1 . \\
3\end{array}$ & $\begin{array}{r}0.6 \\
0\end{array}$ & 955.8 & 11.2 & 977.9 & 13.1 & $\begin{array}{r}1027 . \\
8\end{array}$ & 33.8 & 955.8 & 11.2 & 93.0 \\
\hline $\begin{array}{l}\text { BU0742- } \\
76\end{array}$ & 65 & 19355 & 1.0 & $\begin{array}{r}13.31 \\
79\end{array}$ & 3.5 & 1.6492 & 4.7 & $\begin{array}{r}0.159 \\
3\end{array}$ & $\begin{array}{r}3 . \\
0\end{array}$ & $\begin{array}{r}0.6 \\
5\end{array}$ & 952.9 & 26.8 & 989.2 & 29.5 & $\begin{array}{r}1070 . \\
8\end{array}$ & 71.2 & 1070.8 & 71.2 & 89.0 \\
\hline $\begin{array}{l}\text { BU0742- } \\
63\end{array}$ & 394 & 86120 & 1.8 & $\begin{array}{r}12.84 \\
26\end{array}$ & 2.5 & 2.0282 & 3.5 & $\begin{array}{r}0.188 \\
9\end{array}$ & $\begin{array}{r}2 . \\
5\end{array}$ & $\begin{array}{r}0.7 \\
1\end{array}$ & $\begin{array}{r}1115 . \\
5\end{array}$ & 25.8 & 1125.0 & 24.1 & $\begin{array}{r}1143 . \\
4\end{array}$ & 49.3 & 1143.4 & 49.3 & 97.6 \\
\hline $\begin{array}{l}\text { BU0742- } \\
73\end{array}$ & 769 & $\begin{array}{r}10153 \\
5\end{array}$ & 1.9 & $\begin{array}{r}12.79 \\
42\end{array}$ & 1.9 & 1.9197 & 2.5 & $\begin{array}{r}0.178 \\
1\end{array}$ & $\begin{array}{r}1 . \\
6\end{array}$ & $\begin{array}{r}0.6 \\
4\end{array}$ & $\begin{array}{r}1056 . \\
8\end{array}$ & 15.4 & 1088.0 & 16.5 & $\begin{array}{r}1150 . \\
9\end{array}$ & 37.7 & 1150.9 & 37.7 & 91.8 \\
\hline $\begin{array}{l}\text { BU0742- } \\
33\end{array}$ & 776 & 92680 & 1.2 & $\begin{array}{r}12.65 \\
03 \\
\end{array}$ & 2.3 & 2.0590 & 2.5 & $\begin{array}{r}0.188 \\
9 \\
\end{array}$ & $\begin{array}{r}1 . \\
0\end{array}$ & $\begin{array}{r}0.4 \\
1 \\
\end{array}$ & $\begin{array}{r}1115 . \\
5 \\
\end{array}$ & 10.2 & 1135.3 & 16.8 & $\begin{array}{r}1173 . \\
4 \\
\end{array}$ & 44.5 & 1173.4 & 44.5 & 95.1 \\
\hline
\end{tabular}




\begin{tabular}{|c|c|c|c|c|c|c|c|c|c|c|c|c|c|c|c|c|c|c|c|}
\hline $\begin{array}{l}\text { BU0742- } \\
42\end{array}$ & 775 & $\begin{array}{r}11556 \\
0 \\
\end{array}$ & 1.4 & $\begin{array}{r}12.39 \\
43 \\
\end{array}$ & 4.4 & 1.6194 & 4.8 & $\begin{array}{r}0.145 \\
6 \\
\end{array}$ & $\begin{array}{r}2 . \\
1\end{array}$ & $\begin{array}{r}0.4 \\
4 \\
\end{array}$ & 876.1 & 17.4 & 977.8 & 30.4 & $\begin{array}{r}1213 . \\
7 \\
\end{array}$ & 85.6 & 1213.7 & 85.6 & 72.2 \\
\hline $\begin{array}{l}\text { BU0742- } \\
12\end{array}$ & 76 & 18105 & 1.6 & $\begin{array}{r}12.22 \\
11\end{array}$ & 4.1 & 2.3299 & 4.3 & $\begin{array}{r}0.206 \\
5\end{array}$ & $\begin{array}{r}1 . \\
0\end{array}$ & $\begin{array}{r}0.2 \\
3\end{array}$ & $\begin{array}{r}1210 . \\
2\end{array}$ & 11.0 & 1221.4 & 30.3 & $\begin{array}{r}1241 . \\
3\end{array}$ & 81.2 & 1241.3 & 81.2 & 97.5 \\
\hline $\begin{array}{l}\text { BU0742- } \\
52\end{array}$ & 388 & 31905 & 1.0 & $\begin{array}{r}12.00 \\
99\end{array}$ & 2.0 & 2.4730 & 2.3 & $\begin{array}{r}0.215 \\
4\end{array}$ & $\begin{array}{r}1 . \\
0\end{array}$ & $\begin{array}{r}0.4 \\
4\end{array}$ & $\begin{array}{r}1257 . \\
6\end{array}$ & 11.4 & 1264.2 & 16.4 & $\begin{array}{r}1275 . \\
4\end{array}$ & 39.8 & 1275.4 & 39.8 & 98.6 \\
\hline $\begin{array}{l}\text { BU0742- } \\
26\end{array}$ & 504 & 92890 & 3.5 & $\begin{array}{r}12.00 \\
37 \\
\end{array}$ & 3.7 & 2.3469 & 3.9 & $\begin{array}{r}0.204 \\
3 \\
\end{array}$ & $\begin{array}{r}1 . \\
1\end{array}$ & $\begin{array}{r}0.2 \\
9 \\
\end{array}$ & $\begin{array}{r}1198 . \\
5\end{array}$ & 12.2 & 1226.6 & 27.8 & $\begin{array}{r}1276 . \\
4\end{array}$ & 73.0 & 1276.4 & 73.0 & 93.9 \\
\hline $\begin{array}{l}\text { BU0742- } \\
87\end{array}$ & 370 & 68750 & 1.3 & $\begin{array}{r}11.90 \\
54 \\
\end{array}$ & 3.6 & 2.4024 & 3.7 & $\begin{array}{r}0.207 \\
4 \\
\end{array}$ & $\begin{array}{r}1 . \\
0\end{array}$ & $\begin{array}{r}0.2 \\
7 \\
\end{array}$ & $\begin{array}{r}1215 . \\
2 \\
\end{array}$ & 11.1 & 1243.3 & 26.9 & $\begin{array}{r}1292 . \\
4 \\
\end{array}$ & 70.3 & 1292.4 & 70.3 & 94.0 \\
\hline $\begin{array}{l}\text { BU0742- } \\
99\end{array}$ & 477 & $\begin{array}{r}10192 \\
5 \\
\end{array}$ & 6.1 & $\begin{array}{r}11.80 \\
21 \\
\end{array}$ & 4.2 & 2.4178 & 4.6 & $\begin{array}{r}0.207 \\
0 \\
\end{array}$ & $\begin{array}{r}1 . \\
8\end{array}$ & $\begin{array}{r}0.4 \\
0 \\
\end{array}$ & $\begin{array}{r}1212 . \\
6 \\
\end{array}$ & 20.2 & 1247.9 & 32.9 & $\begin{array}{r}1309 . \\
4\end{array}$ & 81.4 & 1309.4 & 81.4 & 92.6 \\
\hline $\begin{array}{l}\text { BU0742- } \\
46\end{array}$ & 638 & $\begin{array}{r}11365 \\
5\end{array}$ & 0.9 & $\begin{array}{r}11.78 \\
19\end{array}$ & 3.9 & 2.5211 & 4.1 & $\begin{array}{r}0.215 \\
4\end{array}$ & $\begin{array}{r}1 . \\
1\end{array}$ & $\begin{array}{r}0.2 \\
6 \\
\end{array}$ & $\begin{array}{r}1257 . \\
7\end{array}$ & 12.0 & 1278.1 & 29.6 & $\begin{array}{r}1312 . \\
7\end{array}$ & 76.3 & 1312.7 & 76.3 & 95.8 \\
\hline $\begin{array}{l}\text { BU0742- } \\
98\end{array}$ & 411 & $\begin{array}{r}12893 \\
5 \\
\end{array}$ & 1.4 & $\begin{array}{r}11.70 \\
71 \\
\end{array}$ & 2.6 & 2.6386 & 2.9 & $\begin{array}{r}0.224 \\
0\end{array}$ & $\begin{array}{r}1 . \\
3\end{array}$ & $\begin{array}{r}0.4 \\
4\end{array}$ & $\begin{array}{r}1303 . \\
2\end{array}$ & 15.2 & 1311.5 & 21.4 & $\begin{array}{r}1325 . \\
0\end{array}$ & 50.4 & 1325.0 & 50.4 & 98.4 \\
\hline $\begin{array}{l}\text { BU0742- } \\
92\end{array}$ & 468 & 52750 & 1.9 & $\begin{array}{r}11.41 \\
46 \\
\end{array}$ & 2.2 & 2.4262 & 2.4 & $\begin{array}{r}0.200 \\
9 \\
\end{array}$ & $\begin{array}{r}1 . \\
0\end{array}$ & $\begin{array}{r}0.4 \\
1\end{array}$ & $\begin{array}{r}1179 . \\
9\end{array}$ & 10.8 & 1250.4 & 17.4 & $\begin{array}{r}1373 . \\
9\end{array}$ & 42.5 & 1373.9 & 42.5 & 85.9 \\
\hline $\begin{array}{l}\text { BU0742- } \\
19\end{array}$ & 159 & 48135 & 1.1 & $\begin{array}{r}11.31 \\
31 \\
\end{array}$ & 3.6 & 2.9058 & 3.8 & $\begin{array}{r}0.238 \\
4 \\
\end{array}$ & $\begin{array}{r}1 . \\
0\end{array}$ & $\begin{array}{r}0.2 \\
7 \\
\end{array}$ & $\begin{array}{r}1378 . \\
5 \\
\end{array}$ & 12.4 & 1383.4 & 28.4 & $\begin{array}{r}1391 . \\
0 \\
\end{array}$ & 69.5 & 1391.0 & 69.5 & 99.1 \\
\hline $\begin{array}{l}\text { BU0742- } \\
18\end{array}$ & 364 & $\begin{array}{r}10123 \\
0\end{array}$ & 1.1 & $\begin{array}{r}11.17 \\
69\end{array}$ & 3.8 & 2.9232 & 4.1 & $\begin{array}{r}0.237 \\
0\end{array}$ & $\begin{array}{r}1 . \\
4\end{array}$ & $\begin{array}{r}0.3 \\
5\end{array}$ & $\begin{array}{r}1370 . \\
9\end{array}$ & 17.8 & 1387.9 & 30.9 & $\begin{array}{r}1414 . \\
2\end{array}$ & 73.1 & 1414.2 & 73.1 & 96.9 \\
\hline $\begin{array}{l}\text { BU0742- } \\
39\end{array}$ & 319 & 47840 & 1.7 & $\begin{array}{r}11.12 \\
67\end{array}$ & 2.9 & 2.4372 & 3.3 & $\begin{array}{r}0.196 \\
7\end{array}$ & $\begin{array}{l}1 . \\
7\end{array}$ & $\begin{array}{r}0.5 \\
0\end{array}$ & $\begin{array}{r}1157 . \\
5\end{array}$ & 17.5 & 1253.7 & 23.7 & $\begin{array}{r}1422 . \\
8\end{array}$ & 54.5 & 1422.8 & 54.5 & 81.3 \\
\hline $\begin{array}{l}\text { BU0742- } \\
28\end{array}$ & 107 & 18990 & 1.6 & $\begin{array}{r}11.12 \\
18 \\
\end{array}$ & 2.6 & 2.8866 & 2.8 & $\begin{array}{r}0.232 \\
8 \\
\end{array}$ & $\begin{array}{r}1 . \\
1\end{array}$ & $\begin{array}{r}0.3 \\
9 \\
\end{array}$ & $\begin{array}{r}1349 . \\
4\end{array}$ & 13.3 & 1378.4 & 21.2 & $\begin{array}{r}1423 . \\
7\end{array}$ & 49.5 & 1423.7 & 49.5 & 94.8 \\
\hline $\begin{array}{l}\text { BU0742- } \\
79\end{array}$ & 488 & 91400 & 2.9 & $\begin{array}{r}11.11 \\
13 \\
\end{array}$ & 4.9 & 2.7908 & 5.2 & $\begin{array}{r}0.224 \\
9 \\
\end{array}$ & $\begin{array}{r}2 . \\
0\end{array}$ & $\begin{array}{r}0.3 \\
8 \\
\end{array}$ & $\begin{array}{r}1307 . \\
7 \\
\end{array}$ & 23.4 & 1353.1 & 39.2 & $\begin{array}{r}1425 . \\
5 \\
\end{array}$ & 92.9 & 1425.5 & 92.9 & 91.7 \\
\hline $\begin{array}{l}\text { BU0742- } \\
70\end{array}$ & 153 & 35975 & 2.5 & $\begin{array}{r}10.96 \\
63 \\
\end{array}$ & 3.3 & 2.9151 & 3.7 & $\begin{array}{r}0.231 \\
9 \\
\end{array}$ & $\begin{array}{r}1 . \\
8\end{array}$ & $\begin{array}{r}0.4 \\
7 \\
\end{array}$ & $\begin{array}{r}1344 . \\
2 \\
\end{array}$ & 21.2 & 1385.8 & 28.1 & $\begin{array}{r}1450 . \\
5 \\
\end{array}$ & 62.3 & 1450.5 & 62.3 & 92.7 \\
\hline $\begin{array}{l}\text { BU0742- } \\
64\end{array}$ & 302 & $\begin{array}{r}10557 \\
5\end{array}$ & 1.5 & $\begin{array}{r}10.94 \\
02\end{array}$ & 4.0 & 3.1577 & 4.1 & $\begin{array}{r}0.250 \\
6\end{array}$ & $\begin{array}{r}1 . \\
0\end{array}$ & $\begin{array}{r}0.2 \\
4\end{array}$ & $\begin{array}{r}1441 . \\
3\end{array}$ & 12.9 & 1446.9 & 31.6 & $\begin{array}{r}1455 . \\
1\end{array}$ & 75.5 & 1455.1 & 75.5 & 99.1 \\
\hline $\begin{array}{l}\text { BU0742- } \\
51\end{array}$ & 180 & 56940 & 1.1 & $\begin{array}{r}10.75 \\
08 \\
\end{array}$ & 2.4 & 3.2062 & 2.6 & $\begin{array}{r}0.250 \\
0 \\
\end{array}$ & $\begin{array}{r}1 . \\
0\end{array}$ & $\begin{array}{r}0.3 \\
8 \\
\end{array}$ & $\begin{array}{r}1438 . \\
4 \\
\end{array}$ & 12.9 & 1458.7 & 20.3 & $\begin{array}{r}1488 . \\
2 \\
\end{array}$ & 45.8 & 1488.2 & 45.8 & 96.7 \\
\hline $\begin{array}{l}\text { BU0742- } \\
69\end{array}$ & 353 & 39040 & 0.7 & $\begin{array}{r}10.65 \\
36 \\
\end{array}$ & 2.4 & 2.6664 & 3.3 & $\begin{array}{r}0.206 \\
0 \\
\end{array}$ & $\begin{array}{r}2 . \\
3 \\
\end{array}$ & $\begin{array}{r}0.6 \\
8 \\
\end{array}$ & $\begin{array}{r}1207 . \\
6 \\
\end{array}$ & 24.8 & 1319.2 & 24.4 & $\begin{array}{r}1505 . \\
4 \\
\end{array}$ & 45.5 & 1505.4 & 45.5 & 80.2 \\
\hline $\begin{array}{l}\text { BU0742- } \\
29\end{array}$ & 241 & 54180 & 1.0 & $\begin{array}{r}10.46 \\
62 \\
\end{array}$ & 5.1 & 3.3673 & 5.5 & $\begin{array}{r}0.255 \\
6 \\
\end{array}$ & $\begin{array}{r}1 . \\
9\end{array}$ & $\begin{array}{r}0.3 \\
5 \\
\end{array}$ & $\begin{array}{r}1467 . \\
3 \\
\end{array}$ & 25.1 & 1496.8 & 42.9 & $\begin{array}{r}1538 . \\
8 \\
\end{array}$ & 96.6 & 1538.8 & 96.6 & 95.4 \\
\hline $\begin{array}{l}\text { BU0742- } \\
93\end{array}$ & 424 & 35220 & 0.9 & $\begin{array}{r}10.44 \\
64\end{array}$ & 4.6 & 2.7919 & 4.8 & $\begin{array}{r}0.211 \\
5\end{array}$ & $\begin{array}{r}1 . \\
5\end{array}$ & $\begin{array}{r}0.3 \\
2\end{array}$ & $\begin{array}{r}1236 . \\
9\end{array}$ & 17.2 & 1353.4 & 36.2 & $\begin{array}{r}1542 . \\
4\end{array}$ & 86.4 & 1542.4 & 86.4 & 80.2 \\
\hline $\begin{array}{l}\text { BU0742- } \\
25\end{array}$ & 493 & 88135 & 1.8 & $\begin{array}{r}10.39 \\
61\end{array}$ & 2.0 & 3.5552 & 2.2 & $\begin{array}{r}0.268 \\
1\end{array}$ & $\begin{array}{r}1 . \\
0\end{array}$ & $\begin{array}{r}0.4 \\
5\end{array}$ & $\begin{array}{r}1530 . \\
9\end{array}$ & 13.6 & 1539.6 & 17.5 & $\begin{array}{r}1551 . \\
5\end{array}$ & 37.0 & 1551.5 & 37.0 & 98.7 \\
\hline BU0742-9 & 191 & 35275 & 1.2 & $\begin{array}{r}10.38 \\
06\end{array}$ & 3.4 & 3.0222 & 4.0 & $\begin{array}{r}0.227 \\
5\end{array}$ & $\begin{array}{r}2 . \\
1\end{array}$ & $\begin{array}{r}0.5 \\
2\end{array}$ & $\begin{array}{r}1321 . \\
6\end{array}$ & 24.5 & 1413.2 & 30.4 & $\begin{array}{r}1554 . \\
3\end{array}$ & 64.0 & 1554.3 & 64.0 & 85.0 \\
\hline $\begin{array}{l}\text { BU0742- } \\
35\end{array}$ & 94 & 23470 & 1.1 & $\begin{array}{r}10.17 \\
99\end{array}$ & 2.2 & 3.5812 & 2.4 & $\begin{array}{r}0.264 \\
4\end{array}$ & $\begin{array}{r}1 . \\
1\end{array}$ & $\begin{array}{r}0.4 \\
5\end{array}$ & $\begin{array}{r}1512 . \\
3\end{array}$ & 14.7 & 1545.4 & 19.2 & $\begin{array}{r}1590 . \\
8\end{array}$ & 40.4 & 1590.8 & 40.4 & 95.1 \\
\hline $\begin{array}{l}\text { BU0742- } \\
15\end{array}$ & 384 & $\begin{array}{r}11803 \\
0 \\
\end{array}$ & 0.9 & $\begin{array}{r}10.14 \\
66 \\
\end{array}$ & 3.6 & 3.5809 & 4.1 & $\begin{array}{r}0.263 \\
5 \\
\end{array}$ & $\begin{array}{r}1 . \\
8 \\
\end{array}$ & $\begin{array}{r}0.4 \\
4 \\
\end{array}$ & $\begin{array}{r}1507 . \\
8 \\
\end{array}$ & 24.1 & 1545.3 & 32.2 & $\begin{array}{r}1597 . \\
0 \\
\end{array}$ & 68.0 & 1597.0 & 68.0 & 94.4 \\
\hline BU0742-5 & 248 & 11053 & 1.8 & 10.05 & 5.1 & 3.6293 & 5.7 & 0.264 & 2. & 0.4 & 1514. & 35.4 & 1556.0 & 45.7 & 1613. & 95.1 & 1613.0 & 95.1 & 93.9 \\
\hline
\end{tabular}




\begin{tabular}{|c|c|c|c|c|c|c|c|c|c|c|c|c|c|c|c|c|c|c|c|}
\hline & & 0 & & 94 & & & & 8 & 6 & 6 & 3 & & & & 0 & & & & \\
\hline $\begin{array}{l}\text { BU0742- } \\
72\end{array}$ & 284 & 49920 & 4.6 & $\begin{array}{r}10.00 \\
72 \\
\end{array}$ & 2.0 & 3.7408 & 3.0 & $\begin{array}{r}0.271 \\
5 \\
\end{array}$ & $\begin{array}{r}2 . \\
2\end{array}$ & $\begin{array}{r}0.7 \\
5\end{array}$ & $\begin{array}{r}1548 . \\
4\end{array}$ & 30.6 & 1580.1 & 23.7 & $\begin{array}{r}1622 . \\
7\end{array}$ & 36.3 & 1622.7 & 36.3 & 95.4 \\
\hline $\begin{array}{l}\text { BU0742- } \\
96\end{array}$ & 948 & $\begin{array}{r}37015 \\
5 \\
\end{array}$ & 2.9 & $\begin{array}{r}10.00 \\
54 \\
\end{array}$ & 4.1 & 3.3697 & 4.3 & $\begin{array}{r}0.244 \\
5 \\
\end{array}$ & $\begin{array}{r}1 . \\
3\end{array}$ & $\begin{array}{r}0.3 \\
1 \\
\end{array}$ & $\begin{array}{r}1410 . \\
2 \\
\end{array}$ & 17.0 & 1497.4 & 33.9 & $\begin{array}{r}1623 . \\
1 \\
\end{array}$ & 76.7 & 1623.1 & 76.7 & 86.9 \\
\hline $\begin{array}{l}\text { BU0742- } \\
95\end{array}$ & 344 & 87890 & 0.8 & $\begin{array}{r}10.00 \\
39 \\
\end{array}$ & 1.7 & 3.6529 & 2.0 & $\begin{array}{r}0.265 \\
0 \\
\end{array}$ & $\begin{array}{r}1 . \\
0\end{array}$ & $\begin{array}{r}0.5 \\
1 \\
\end{array}$ & $\begin{array}{r}1515 . \\
6 \\
\end{array}$ & 13.5 & 1561.1 & 15.7 & $\begin{array}{r}1623 . \\
3\end{array}$ & 31.4 & 1623.3 & 31.4 & 93.4 \\
\hline $\begin{array}{l}\text { BU0742- } \\
55\end{array}$ & 888 & $\begin{array}{r}11448 \\
5 \\
\end{array}$ & 5.9 & $\begin{array}{r}9.991 \\
0 \\
\end{array}$ & 5.7 & 3.6311 & 6.4 & $\begin{array}{r}0.263 \\
1 \\
\end{array}$ & $\begin{array}{r}3 . \\
0\end{array}$ & $\begin{array}{r}0.4 \\
6 \\
\end{array}$ & $\begin{array}{r}1505 . \\
8 \\
\end{array}$ & 40.0 & 1556.4 & 51.3 & $\begin{array}{r}1625 . \\
7 \\
\end{array}$ & $\begin{array}{r}106 . \\
1 \\
\end{array}$ & 1625.7 & $\begin{array}{r}106 . \\
1 \\
\end{array}$ & 92.6 \\
\hline $\begin{array}{l}\text { BU0742- } \\
58\end{array}$ & 170 & 44170 & 0.9 & $\begin{array}{r}9.984 \\
3 \\
\end{array}$ & 3.7 & 3.7580 & 5.0 & $\begin{array}{r}0.272 \\
1 \\
\end{array}$ & $\begin{array}{r}3 . \\
4 \\
\end{array}$ & $\begin{array}{r}0.6 \\
7 \\
\end{array}$ & $\begin{array}{r}1551 . \\
6 \\
\end{array}$ & 46.5 & 1583.8 & 40.1 & $\begin{array}{r}1627 . \\
0 \\
\end{array}$ & 68.6 & 1627.0 & 68.6 & 95.4 \\
\hline BU0742-3 & 513 & $\begin{array}{r}10542 \\
0 \\
\end{array}$ & 3.5 & $\begin{array}{r}9.949 \\
8 \\
\end{array}$ & 2.0 & 3.8723 & 2.3 & $\begin{array}{r}0.279 \\
4 \\
\end{array}$ & $\begin{array}{c}1 . \\
0\end{array}$ & $\begin{array}{r}0.4 \\
4 \\
\end{array}$ & $\begin{array}{r}1588 . \\
5 \\
\end{array}$ & 14.1 & 1607.9 & 18.3 & $\begin{array}{r}1633 . \\
4 \\
\end{array}$ & 37.7 & 1633.4 & 37.7 & 97.3 \\
\hline $\begin{array}{l}\text { BU0742- } \\
61\end{array}$ & 214 & 42420 & 0.8 & $\begin{array}{r}9.918 \\
1 \\
\end{array}$ & 4.6 & 3.9339 & 4.7 & $\begin{array}{r}0.283 \\
0 \\
\end{array}$ & $\begin{array}{c}1 . \\
1\end{array}$ & $\begin{array}{r}0.2 \\
4 \\
\end{array}$ & $\begin{array}{r}1606 . \\
3 \\
\end{array}$ & 16.1 & 1620.7 & 38.2 & $\begin{array}{r}1639 . \\
4 \\
\end{array}$ & 85.1 & 1639.4 & 85.1 & 98.0 \\
\hline $\begin{array}{l}\text { BU0742- } \\
56\end{array}$ & 567 & 79280 & 1.7 & $\begin{array}{r}9.843 \\
7 \\
\end{array}$ & 3.9 & 3.5328 & 4.2 & $\begin{array}{r}0.252 \\
2 \\
\end{array}$ & $\begin{array}{c}1 . \\
5\end{array}$ & $\begin{array}{r}0.3 \\
7 \\
\end{array}$ & $\begin{array}{r}1449 . \\
9 \\
\end{array}$ & 20.0 & 1534.6 & 33.0 & $\begin{array}{r}1653 . \\
3 \\
\end{array}$ & 71.7 & 1653.3 & 71.7 & 87.7 \\
\hline BU0742-1 & 781 & $\begin{array}{r}15728 \\
5 \\
\end{array}$ & 1.6 & $\begin{array}{r}9.833 \\
4 \\
\end{array}$ & 2.6 & 4.0000 & 2.8 & $\begin{array}{r}0.285 \\
3 \\
\end{array}$ & $\begin{array}{r}1 . \\
0\end{array}$ & $\begin{array}{r}0.3 \\
6 \\
\end{array}$ & $\begin{array}{r}1617 . \\
9 \\
\end{array}$ & 14.3 & 1634.2 & 22.4 & $\begin{array}{r}1655 . \\
2 \\
\end{array}$ & 47.6 & 1655.2 & 47.6 & 97.7 \\
\hline $\begin{array}{l}\text { BU0742- } \\
16\end{array}$ & 126 & 83425 & 1.1 & $\begin{array}{r}9.772 \\
0 \\
\end{array}$ & 2.5 & 4.0866 & 3.2 & $\begin{array}{r}0.289 \\
6 \\
\end{array}$ & $\begin{array}{r}2 . \\
0\end{array}$ & $\begin{array}{r}0.6 \\
3 \\
\end{array}$ & $\begin{array}{r}1639 . \\
7\end{array}$ & 29.4 & 1651.6 & 26.3 & $\begin{array}{r}1666 . \\
9\end{array}$ & 46.4 & 1666.9 & 46.4 & 98.4 \\
\hline $\begin{array}{l}\text { BU0742- } \\
10\end{array}$ & $\begin{array}{r}175 \\
2 \\
\end{array}$ & $\begin{array}{r}24353 \\
5 \\
\end{array}$ & 2.8 & $\begin{array}{r}9.718 \\
3 \\
\end{array}$ & 3.0 & 3.8050 & 5.6 & $\begin{array}{r}0.268 \\
2 \\
\end{array}$ & $\begin{array}{l}4 . \\
7 \\
\end{array}$ & $\begin{array}{r}0.8 \\
5 \\
\end{array}$ & $\begin{array}{r}1531 . \\
6 \\
\end{array}$ & 64.5 & 1593.8 & 45.0 & $\begin{array}{r}1677 . \\
0 \\
\end{array}$ & 55.3 & 1677.0 & 55.3 & 91.3 \\
\hline $\begin{array}{l}\text { BU0742- } \\
83\end{array}$ & 36 & 17640 & 1.5 & $\begin{array}{r}9.633 \\
7 \\
\end{array}$ & 6.6 & 4.0738 & 7.4 & $\begin{array}{r}0.284 \\
6 \\
\end{array}$ & $\begin{array}{l}3 . \\
3\end{array}$ & $\begin{array}{r}0.4 \\
5 \\
\end{array}$ & $\begin{array}{r}1614 . \\
7\end{array}$ & 47.4 & 1649.1 & 60.3 & $\begin{array}{r}1693 . \\
2 \\
\end{array}$ & $\begin{array}{r}121 . \\
8 \\
\end{array}$ & 1693.2 & $\begin{array}{r}121 . \\
8 \\
\end{array}$ & 95.4 \\
\hline $\begin{array}{l}\text { BU0742- } \\
59\end{array}$ & 267 & 56280 & 1.2 & $\begin{array}{r}9.625 \\
7 \\
\end{array}$ & 3.9 & 3.1373 & 7.2 & $\begin{array}{r}0.219 \\
0 \\
\end{array}$ & $\begin{array}{r}6 . \\
0 \\
\end{array}$ & $\begin{array}{r}0.8 \\
4 \\
\end{array}$ & $\begin{array}{r}1276 . \\
7 \\
\end{array}$ & 69.5 & 1441.9 & 55.3 & $\begin{array}{r}1694 . \\
7 \\
\end{array}$ & 72.5 & 1694.7 & 72.5 & 75.3 \\
\hline $\begin{array}{l}\text { BU0742- } \\
45\end{array}$ & 898 & $\begin{array}{r}21150 \\
5 \\
\end{array}$ & 1.7 & $\begin{array}{r}9.567 \\
4 \\
\end{array}$ & 4.6 & 4.2728 & 5.0 & $\begin{array}{r}0.296 \\
5\end{array}$ & $\begin{array}{c}1 . \\
9\end{array}$ & $\begin{array}{r}0.3 \\
9 \\
\end{array}$ & $\begin{array}{r}1673 . \\
9 \\
\end{array}$ & 28.5 & 1688.1 & 41.1 & $\begin{array}{r}1705 . \\
9\end{array}$ & 84.7 & 1705.9 & 84.7 & 98.1 \\
\hline $\begin{array}{l}\text { BU0742- } \\
74\end{array}$ & 902 & $\begin{array}{r}15351 \\
5 \\
\end{array}$ & 1.7 & $\begin{array}{r}9.546 \\
6 \\
\end{array}$ & 2.2 & 4.0313 & 2.4 & $\begin{array}{r}0.279 \\
1 \\
\end{array}$ & $\begin{array}{r}1 . \\
0\end{array}$ & $\begin{array}{r}0.4 \\
3 \\
\end{array}$ & $\begin{array}{r}1586 . \\
9 \\
\end{array}$ & 14.5 & 1640.5 & 19.6 & $\begin{array}{r}1709 . \\
9 \\
\end{array}$ & 40.1 & 1709.9 & 40.1 & 92.8 \\
\hline $\begin{array}{l}\text { BU0742- } \\
44\end{array}$ & 542 & $\begin{array}{r}11372 \\
0 \\
\end{array}$ & 1.4 & $\begin{array}{r}9.545 \\
3 \\
\end{array}$ & 4.6 & 4.2719 & 4.7 & $\begin{array}{r}0.295 \\
7 \\
\end{array}$ & $\begin{array}{c}1 . \\
1\end{array}$ & $\begin{array}{r}0.2 \\
3 \\
\end{array}$ & $\begin{array}{r}1670 . \\
1 \\
\end{array}$ & 15.7 & 1688.0 & 38.5 & $\begin{array}{r}1710 . \\
2 \\
\end{array}$ & 83.8 & 1710.2 & 83.8 & 97.7 \\
\hline $\begin{array}{l}\text { BU0742- } \\
37\end{array}$ & 281 & $\begin{array}{r}10175 \\
0 \\
\end{array}$ & 1.5 & $\begin{array}{r}9.531 \\
9 \\
\end{array}$ & 4.0 & 4.2875 & 4.1 & $\begin{array}{r}0.296 \\
4 \\
\end{array}$ & $\begin{array}{c}. \\
0\end{array}$ & $\begin{array}{r}0.2 \\
4 \\
\end{array}$ & $\begin{array}{r}1673 . \\
5 \\
\end{array}$ & 14.7 & 1691.0 & 34.0 & $\begin{array}{r}1712 . \\
7 \\
\end{array}$ & 73.6 & 1712.7 & 73.6 & 97.7 \\
\hline $\begin{array}{l}\text { BU0742- } \\
49\end{array}$ & $\begin{array}{r}137 \\
2 \\
\end{array}$ & $\begin{array}{r}15127 \\
5 \\
\end{array}$ & 1.9 & $\begin{array}{r}9.510 \\
7 \\
\end{array}$ & 1.8 & 4.0288 & 2.1 & $\begin{array}{r}0.277 \\
9 \\
\end{array}$ & $\begin{array}{r}1 . \\
0\end{array}$ & $\begin{array}{r}0.4 \\
8 \\
\end{array}$ & $\begin{array}{r}1580 . \\
8 \\
\end{array}$ & 14.0 & 1640.0 & 17.0 & $\begin{array}{r}1716 . \\
8 \\
\end{array}$ & 33.6 & 1716.8 & 33.6 & 92.1 \\
\hline $\begin{array}{l}\text { BU0742- } \\
67\end{array}$ & 598 & 50220 & 0.8 & $\begin{array}{r}9.492 \\
6 \\
\end{array}$ & 1.8 & 3.7055 & 2.0 & $\begin{array}{r}0.255 \\
1 \\
\end{array}$ & $\begin{array}{c}1 . \\
0\end{array}$ & $\begin{array}{r}0.4 \\
9 \\
\end{array}$ & $\begin{array}{r}1464 . \\
8 \\
\end{array}$ & 13.1 & 1572.6 & 16.3 & $\begin{array}{r}1720 . \\
3 \\
\end{array}$ & 32.5 & 1720.3 & 32.5 & 85.1 \\
\hline $\begin{array}{l}\text { BU0742- } \\
43\end{array}$ & $\begin{array}{r}116 \\
4 \\
\end{array}$ & $\begin{array}{r}21205 \\
5 \\
\end{array}$ & 1.6 & $\begin{array}{r}9.468 \\
1 \\
\end{array}$ & 3.6 & 4.1976 & 3.8 & $\begin{array}{r}0.288 \\
2 \\
\end{array}$ & $\begin{array}{c}1 . \\
3\end{array}$ & $\begin{array}{r}0.3 \\
3 \\
\end{array}$ & $\begin{array}{r}1632 . \\
7 \\
\end{array}$ & 18.5 & 1673.5 & 31.5 & $\begin{array}{r}1725 . \\
1 \\
\end{array}$ & 66.5 & 1725.1 & 66.5 & 94.6 \\
\hline $\begin{array}{l}\text { BU0742- } \\
24\end{array}$ & 627 & $\begin{array}{r}13521 \\
5 \\
\end{array}$ & 1.5 & $\begin{array}{r}9.466 \\
6 \\
\end{array}$ & 1.7 & 4.0698 & 2.0 & $\begin{array}{r}0.279 \\
4 \\
\end{array}$ & $\begin{array}{r}1 . \\
1 \\
\end{array}$ & $\begin{array}{r}0.5 \\
4 \\
\end{array}$ & $\begin{array}{r}1588 . \\
5 \\
\end{array}$ & 15.2 & 1648.3 & 16.4 & $\begin{array}{r}1725 . \\
4 \\
\end{array}$ & 31.2 & 1725.4 & 31.2 & 92.1 \\
\hline $\begin{array}{l}\text { BU0742- } \\
20\end{array}$ & 721 & $\begin{array}{r}19103 \\
0 \\
\end{array}$ & 1.8 & $\begin{array}{r}9.462 \\
2 \\
\end{array}$ & 5.9 & 3.9831 & 6.3 & $\begin{array}{r}0.273 \\
3 \\
\end{array}$ & $\begin{array}{r}2 . \\
0\end{array}$ & $\begin{array}{r}0.3 \\
2 \\
\end{array}$ & $\begin{array}{r}1557 . \\
8 \\
\end{array}$ & 28.0 & 1630.8 & 50.8 & $\begin{array}{r}1726 . \\
2 \\
\end{array}$ & $\begin{array}{r}108 . \\
8 \\
\end{array}$ & 1726.2 & $\begin{array}{r}108 . \\
8 \\
\end{array}$ & 90.2 \\
\hline $\begin{array}{l}\text { BU0742- } \\
31\end{array}$ & $\begin{array}{r}107 \\
7 \\
\end{array}$ & 65580 & 4.3 & $\begin{array}{r}9.458 \\
5 \\
\end{array}$ & 2.5 & 3.9587 & 2.7 & $\begin{array}{r}0.271 \\
6 \\
\end{array}$ & $\begin{array}{l}1 . \\
0\end{array}$ & $\begin{array}{r}0.3 \\
8 \\
\end{array}$ & $\begin{array}{r}1548 . \\
7 \\
\end{array}$ & 13.8 & 1625.8 & 21.5 & $\begin{array}{r}1726 . \\
9\end{array}$ & 45.2 & 1726.9 & 45.2 & 89.7 \\
\hline $\begin{array}{l}\text { BU0742- } \\
53\end{array}$ & 355 & 46685 & 1.2 & $\begin{array}{r}9.458 \\
2 \\
\end{array}$ & 1.6 & 3.4401 & 2.6 & $\begin{array}{r}0.236 \\
0 \\
\end{array}$ & $\begin{array}{r}2 . \\
0\end{array}$ & $\begin{array}{r}0.8 \\
0 \\
\end{array}$ & $\begin{array}{r}1365 . \\
8 \\
\end{array}$ & 25.1 & 1513.6 & 20.2 & $\begin{array}{r}1727 . \\
0 \\
\end{array}$ & 28.5 & 1727.0 & 28.5 & 79.1 \\
\hline
\end{tabular}




\begin{tabular}{|c|c|c|c|c|c|c|c|c|c|c|c|c|c|c|c|c|c|c|c|}
\hline $\begin{array}{l}\text { BU0742- } \\
23\end{array}$ & 173 & 35310 & 1.8 & $\begin{array}{r}9.446 \\
5 \\
\end{array}$ & 1.7 & 4.3282 & 1.9 & $\begin{array}{r}0.296 \\
5 \\
\end{array}$ & $\begin{array}{l}1 . \\
0\end{array}$ & $\begin{array}{r}0.5 \\
2 \\
\end{array}$ & $\begin{array}{r}1674 . \\
1 \\
\end{array}$ & 14.7 & 1698.7 & 15.9 & $\begin{array}{r}1729 . \\
3 \\
\end{array}$ & 30.3 & 1729.3 & 30.3 & 96.8 \\
\hline $\begin{array}{l}\text { BU0742- } \\
68\end{array}$ & 615 & $\begin{array}{r}13118 \\
0\end{array}$ & 0.7 & $\begin{array}{r}9.385 \\
3\end{array}$ & 1.8 & 4.1419 & 3.0 & $\begin{array}{r}0.281 \\
9\end{array}$ & $\begin{array}{r}2 . \\
4\end{array}$ & $\begin{array}{r}0.7 \\
9\end{array}$ & $\begin{array}{r}1601 . \\
1\end{array}$ & 34.2 & 1662.6 & 24.8 & $\begin{array}{r}1741 . \\
2\end{array}$ & 33.7 & 1741.2 & 33.7 & 92.0 \\
\hline $\begin{array}{l}\text { BU0742- } \\
62\end{array}$ & 992 & $\begin{array}{r}22654 \\
5\end{array}$ & 1.9 & $\begin{array}{r}9.380 \\
3\end{array}$ & 1.8 & 4.0772 & 2.5 & $\begin{array}{r}0.277 \\
4\end{array}$ & $\begin{array}{l}1 . \\
7\end{array}$ & $\begin{array}{r}0.6 \\
7\end{array}$ & $\begin{array}{r}1578 . \\
2\end{array}$ & 23.4 & 1649.8 & 20.3 & $\begin{array}{r}1742 . \\
2\end{array}$ & 33.7 & 1742.2 & 33.7 & 90.6 \\
\hline $\begin{array}{l}\text { BU0742- } \\
57\end{array}$ & 222 & 41305 & 1.3 & $\begin{array}{r}9.378 \\
3 \\
\end{array}$ & 2.8 & 3.3898 & 4.7 & $\begin{array}{r}0.230 \\
6 \\
\end{array}$ & $\begin{array}{r}3 . \\
8\end{array}$ & $\begin{array}{r}0.8 \\
0 \\
\end{array}$ & $\begin{array}{r}1337 . \\
5 \\
\end{array}$ & 45.8 & 1502.0 & 37.0 & $\begin{array}{r}1742 . \\
6\end{array}$ & 51.3 & 1742.6 & 51.3 & 76.8 \\
\hline $\begin{array}{l}\text { BU0742- } \\
41\end{array}$ & 212 & 89070 & 0.8 & $\begin{array}{r}9.373 \\
0 \\
\end{array}$ & 3.2 & 4.2349 & 3.4 & $\begin{array}{r}0.287 \\
9 \\
\end{array}$ & $\begin{array}{r}1 . \\
0\end{array}$ & $\begin{array}{r}0.3 \\
0 \\
\end{array}$ & $\begin{array}{r}1631 . \\
0 \\
\end{array}$ & 14.8 & 1680.8 & 27.9 & $\begin{array}{r}1743 . \\
6 \\
\end{array}$ & 59.4 & 1743.6 & 59.4 & 93.5 \\
\hline $\begin{array}{l}\text { BU0742- } \\
100\end{array}$ & 398 & $\begin{array}{r}12817 \\
5 \\
\end{array}$ & 1.8 & $\begin{array}{r}9.364 \\
2 \\
\end{array}$ & 2.9 & 4.0861 & 3.0 & $\begin{array}{r}0.277 \\
5 \\
\end{array}$ & $\begin{array}{r}1 . \\
0\end{array}$ & $\begin{array}{r}0.3 \\
3 \\
\end{array}$ & $\begin{array}{r}1578 . \\
8 \\
\end{array}$ & 14.0 & 1651.5 & 24.6 & $\begin{array}{r}1745 . \\
3\end{array}$ & 52.2 & 1745.3 & 52.2 & 90.5 \\
\hline BU0742-2 & 272 & 53380 & 2.0 & $\begin{array}{r}9.358 \\
1\end{array}$ & 2.9 & 3.9953 & 3.0 & $\begin{array}{r}0.271 \\
2\end{array}$ & $\begin{array}{r}1 . \\
0\end{array}$ & $\begin{array}{r}0.3 \\
3\end{array}$ & $\begin{array}{r}1546 . \\
7\end{array}$ & 13.8 & 1633.2 & 24.5 & $\begin{array}{r}1746 . \\
5\end{array}$ & 52.2 & 1746.5 & 52.2 & 88.6 \\
\hline BU0742-7 & 738 & $\begin{array}{r}12807 \\
0 \\
\end{array}$ & 1.9 & $\begin{array}{r}9.338 \\
9 \\
\end{array}$ & 4.4 & 3.7880 & 4.7 & $\begin{array}{r}0.256 \\
6 \\
\end{array}$ & $\begin{array}{r}1 . \\
8 \\
\end{array}$ & $\begin{array}{r}0.3 \\
7 \\
\end{array}$ & $\begin{array}{r}1472 . \\
3 \\
\end{array}$ & 23.2 & 1590.2 & 38.0 & $\begin{array}{r}1750 . \\
3 \\
\end{array}$ & 80.4 & 1750.3 & 80.4 & 84.1 \\
\hline $\begin{array}{l}\text { BU0742- } \\
80\end{array}$ & 365 & 74205 & 1.4 & $\begin{array}{r}9.300 \\
2 \\
\end{array}$ & 4.6 & 4.2205 & 4.7 & $\begin{array}{r}0.284 \\
7 \\
\end{array}$ & $\begin{array}{c}1 . \\
0\end{array}$ & $\begin{array}{r}0.2 \\
1 \\
\end{array}$ & $\begin{array}{r}1614 . \\
9 \\
\end{array}$ & 14.3 & 1678.0 & 39.0 & $\begin{array}{r}1757 . \\
9 \\
\end{array}$ & 84.9 & 1757.9 & 84.9 & 91.9 \\
\hline $\begin{array}{l}\text { BU0742- } \\
14\end{array}$ & 433 & $\begin{array}{r}13021 \\
5 \\
\end{array}$ & 1.8 & $\begin{array}{r}9.295 \\
6 \\
\end{array}$ & 4.9 & 3.6493 & 5.6 & $\begin{array}{r}0.246 \\
0 \\
\end{array}$ & $\begin{array}{r}2 . \\
7 \\
\end{array}$ & $\begin{array}{r}0.4 \\
9 \\
\end{array}$ & $\begin{array}{r}1418 . \\
0 \\
\end{array}$ & 34.9 & 1560.4 & 44.8 & $\begin{array}{r}1758 . \\
8 \\
\end{array}$ & 89.8 & 1758.8 & 89.8 & 80.6 \\
\hline $\begin{array}{l}\text { BU0742- } \\
17\end{array}$ & 232 & 80910 & 2.0 & $\begin{array}{r}9.273 \\
4\end{array}$ & 5.4 & 4.3050 & 5.5 & $\begin{array}{r}0.289 \\
5\end{array}$ & $\begin{array}{r}1 . \\
0\end{array}$ & $\begin{array}{r}0.1 \\
8\end{array}$ & $\begin{array}{r}1639 . \\
2\end{array}$ & 14.5 & 1694.3 & 45.5 & $\begin{array}{r}1763 . \\
2\end{array}$ & 99.3 & 1763.2 & 99.3 & 93.0 \\
\hline $\begin{array}{l}\text { BU0742- } \\
40\end{array}$ & 110 & 27215 & 0.8 & $\begin{array}{r}9.240 \\
7\end{array}$ & 4.3 & 4.6677 & 4.4 & $\begin{array}{r}0.312 \\
8\end{array}$ & $\begin{array}{r}1 . \\
2\end{array}$ & $\begin{array}{r}0.2 \\
7 \\
\end{array}$ & $\begin{array}{r}1754 . \\
6\end{array}$ & 18.3 & 1761.5 & 37.1 & $\begin{array}{r}1769 . \\
6\end{array}$ & 78.0 & 1769.6 & 78.0 & 99.2 \\
\hline $\begin{array}{l}\text { BU0742- } \\
66\end{array}$ & 323 & 78180 & 1.4 & $\begin{array}{r}9.214 \\
9 \\
\end{array}$ & 2.2 & 4.5134 & 2.8 & $\begin{array}{r}0.301 \\
6 \\
\end{array}$ & $\begin{array}{l}1 . \\
7 \\
\end{array}$ & $\begin{array}{r}0.6 \\
3 \\
\end{array}$ & $\begin{array}{r}1699 . \\
4 \\
\end{array}$ & 25.8 & 1733.4 & 23.0 & $\begin{array}{r}1774 . \\
7 \\
\end{array}$ & 39.4 & 1774.7 & 39.4 & 95.8 \\
\hline BU0742-8 & 642 & 66430 & 1.9 & $\begin{array}{r}9.202 \\
8 \\
\end{array}$ & 4.0 & 4.1943 & 4.2 & $\begin{array}{r}0.279 \\
9 \\
\end{array}$ & $\begin{array}{r}1 . \\
0\end{array}$ & $\begin{array}{r}0.2 \\
4 \\
\end{array}$ & $\begin{array}{r}1591 . \\
1 \\
\end{array}$ & 14.1 & 1672.9 & 34.1 & $\begin{array}{r}1777 . \\
1 \\
\end{array}$ & 73.6 & 1777.1 & 73.6 & 89.5 \\
\hline $\begin{array}{l}\text { BU0742- } \\
78\end{array}$ & 193 & 56105 & 2.1 & $\begin{array}{r}9.180 \\
7 \\
\end{array}$ & 4.8 & 4.0968 & 4.9 & $\begin{array}{r}0.272 \\
8 \\
\end{array}$ & $\begin{array}{r}1 . \\
3\end{array}$ & $\begin{array}{r}0.2 \\
6 \\
\end{array}$ & $\begin{array}{r}1554 . \\
9 \\
\end{array}$ & 17.7 & 1653.7 & 40.4 & $\begin{array}{r}1781 . \\
5 \\
\end{array}$ & 87.2 & 1781.5 & 87.2 & 87.3 \\
\hline $\begin{array}{l}\text { BU0742- } \\
94\end{array}$ & 189 & 31165 & 1.9 & $\begin{array}{r}9.026 \\
0 \\
\end{array}$ & 3.9 & 4.6522 & 4.0 & $\begin{array}{r}0.304 \\
5 \\
\end{array}$ & $\begin{array}{r}1 . \\
0\end{array}$ & $\begin{array}{r}0.2 \\
5\end{array}$ & $\begin{array}{r}1713 . \\
8\end{array}$ & 15.0 & 1758.7 & 33.3 & $\begin{array}{r}1812 . \\
4\end{array}$ & 70.0 & 1812.4 & 70.0 & 94.6 \\
\hline BU0742-4 & 181 & 49180 & 1.7 & $\begin{array}{r}8.868 \\
6 \\
\end{array}$ & 3.6 & 4.7515 & 4.4 & $\begin{array}{r}0.305 \\
6 \\
\end{array}$ & $\begin{array}{r}2 . \\
5\end{array}$ & $\begin{array}{r}0.5 \\
7 \\
\end{array}$ & $\begin{array}{r}1719 . \\
1 \\
\end{array}$ & 38.0 & 1776.4 & 36.9 & $\begin{array}{r}1844 . \\
3 \\
\end{array}$ & 65.2 & 1844.3 & 65.2 & 93.2 \\
\hline $\begin{array}{l}\text { BU0742- } \\
84\end{array}$ & 466 & 69985 & 2.5 & $\begin{array}{r}8.824 \\
3 \\
\end{array}$ & 4.1 & 4.7444 & 4.3 & $\begin{array}{r}0.303 \\
6 \\
\end{array}$ & $\begin{array}{r}1 . \\
4 \\
\end{array}$ & $\begin{array}{r}0.3 \\
3 \\
\end{array}$ & $\begin{array}{r}1709 . \\
3 \\
\end{array}$ & 21.3 & 1775.1 & 36.5 & $\begin{array}{r}1853 . \\
4 \\
\end{array}$ & 74.3 & 1853.4 & 74.3 & 92.2 \\
\hline $\begin{array}{l}\text { BU0742- } \\
36\end{array}$ & 354 & $\begin{array}{r}15407 \\
5 \\
\end{array}$ & 2.1 & $\begin{array}{r}8.804 \\
9 \\
\end{array}$ & 2.6 & 4.2696 & 2.8 & $\begin{array}{r}0.272 \\
7 \\
\end{array}$ & $\begin{array}{r}1 . \\
0 \\
\end{array}$ & $\begin{array}{r}0.3 \\
6 \\
\end{array}$ & $\begin{array}{r}1554 . \\
3 \\
\end{array}$ & 13.8 & 1687.5 & 22.7 & $\begin{array}{r}1857 . \\
3 \\
\end{array}$ & 46.4 & 1857.3 & 46.4 & 83.7 \\
\hline $\begin{array}{l}\text { BU0742- } \\
81\end{array}$ & 397 & $\begin{array}{r}13115 \\
5\end{array}$ & 3.7 & $\begin{array}{r}8.705 \\
5\end{array}$ & 3.7 & 4.6381 & 5.5 & $\begin{array}{r}0.292 \\
8\end{array}$ & $\begin{array}{r}4 . \\
2\end{array}$ & $\begin{array}{r}0.7 \\
5\end{array}$ & $\begin{array}{r}1655 . \\
7\end{array}$ & 60.9 & 1756.1 & 46.4 & $\begin{array}{r}1877 . \\
8\end{array}$ & 66.0 & 1877.8 & 66.0 & 88.2 \\
\hline $\begin{array}{l}\text { BU0742- } \\
77\end{array}$ & 471 & 5900 & 1.2 & $\begin{array}{r}8.640 \\
3 \\
\end{array}$ & 4.3 & 4.2155 & 6.4 & $\begin{array}{r}0.264 \\
2 \\
\end{array}$ & $\begin{array}{r}4 . \\
8 \\
\end{array}$ & $\begin{array}{r}0.7 \\
4\end{array}$ & $\begin{array}{r}1511 . \\
1\end{array}$ & 64.4 & 1677.0 & 52.9 & $\begin{array}{r}1891 . \\
4\end{array}$ & 77.8 & 1891.4 & 77.8 & 79.9 \\
\hline $\begin{array}{l}\text { BU0742- } \\
27\end{array}$ & 267 & 57875 & 1.7 & $\begin{array}{r}8.639 \\
9\end{array}$ & 4.6 & 5.3453 & 4.7 & $\begin{array}{r}0.334 \\
9\end{array}$ & $\begin{array}{r}1 . \\
0\end{array}$ & $\begin{array}{r}0.2 \\
1\end{array}$ & $\begin{array}{r}1862 . \\
3\end{array}$ & 16.2 & 1876.1 & 40.0 & $\begin{array}{r}1891 . \\
5\end{array}$ & 82.1 & 1891.5 & 82.1 & 98.5 \\
\hline $\begin{array}{l}\text { BU0742- } \\
47\end{array}$ & 394 & 9835 & 1.0 & $\begin{array}{r}8.606 \\
2 \\
\end{array}$ & 3.7 & 4.8041 & 3.8 & $\begin{array}{r}0.299 \\
9 \\
\end{array}$ & $\begin{array}{r}1 . \\
1\end{array}$ & $\begin{array}{r}0.2 \\
9 \\
\end{array}$ & $\begin{array}{r}1690 . \\
6 \\
\end{array}$ & 16.8 & 1785.6 & 32.3 & $\begin{array}{r}1898 . \\
5 \\
\end{array}$ & 66.1 & 1898.5 & 66.1 & 89.1 \\
\hline $\begin{array}{l}\text { BU0742- } \\
60\end{array}$ & 150 & 47710 & 1.7 & $\begin{array}{r}8.569 \\
8 \\
\end{array}$ & 2.6 & 5.1191 & 2.8 & $\begin{array}{r}0.318 \\
2 \\
\end{array}$ & $\begin{array}{r}1 . \\
0\end{array}$ & $\begin{array}{r}0.3 \\
6 \\
\end{array}$ & $\begin{array}{r}1780 . \\
8 \\
\end{array}$ & 15.6 & 1839.3 & 23.7 & $\begin{array}{r}1906 . \\
1 \\
\end{array}$ & 46.7 & 1906.1 & 46.7 & 93.4 \\
\hline BU0742- & 908 & 72735 & 2.5 & 8.407 & 2.6 & 4.1605 & 2.8 & 0.253 & 1. & 0.3 & 1457. & 13.0 & 1666.3 & 23.1 & 1940. & 47.2 & 1940.3 & 47.2 & 75.1 \\
\hline
\end{tabular}




\begin{tabular}{|c|c|c|c|c|c|c|c|c|c|c|c|c|c|c|c|c|c|c|c|}
\hline 21 & & & & 8 & & & & 7 & 0 & 5 & 6 & & & & 3 & & & & \\
\hline $\begin{array}{l}\text { BU0742- } \\
32\end{array}$ & $\begin{array}{r}199 \\
9\end{array}$ & $\begin{array}{r}34035 \\
5\end{array}$ & 55.7 & $\begin{array}{r}6.270 \\
8\end{array}$ & 1.5 & 8.1911 & 1.8 & $\begin{array}{r}0.372 \\
5\end{array}$ & $\begin{array}{l}1 . \\
0\end{array}$ & $\begin{array}{r}0.5 \\
5\end{array}$ & $\begin{array}{r}2041 . \\
3\end{array}$ & 17.5 & 2252.4 & 16.4 & $\begin{array}{r}2450 . \\
0\end{array}$ & 25.5 & 2450.0 & 25.5 & 83.3 \\
\hline $\begin{array}{l}\text { BU0742- } \\
30\end{array}$ & 251 & 70545 & 1.1 & $\begin{array}{r}6.179 \\
7\end{array}$ & 2.7 & 9.8910 & 2.9 & $\begin{array}{r}0.443 \\
3\end{array}$ & $\begin{array}{r}1 . \\
0\end{array}$ & $\begin{array}{r}0.3 \\
5\end{array}$ & $\begin{array}{r}2365 . \\
4\end{array}$ & 19.8 & 2424.7 & 26.4 & $\begin{array}{r}2474 . \\
8\end{array}$ & 45.2 & 2474.8 & 45.2 & 95.6 \\
\hline $\begin{array}{l}\text { BU0742- } \\
86\end{array}$ & 162 & 83930 & 2.0 & $\begin{array}{r}6.084 \\
3\end{array}$ & 2.6 & $\begin{array}{r}10.149 \\
3\end{array}$ & 2.7 & $\begin{array}{r}0.447 \\
9\end{array}$ & $\begin{array}{l}1 . \\
0\end{array}$ & $\begin{array}{r}0.3 \\
7\end{array}$ & $\begin{array}{r}2385 . \\
7\end{array}$ & 19.9 & 2448.5 & 25.3 & $\begin{array}{r}2501 . \\
0\end{array}$ & 42.9 & 2501.0 & 42.9 & 95.4 \\
\hline $\begin{array}{l}\text { BU0742- } \\
48\end{array}$ & 301 & 55075 & 0.8 & $\begin{array}{r}6.048 \\
2 \\
\end{array}$ & 2.4 & 9.2749 & 3.4 & $\begin{array}{r}0.406 \\
9 \\
\end{array}$ & $\begin{array}{r}2 . \\
4 \\
\end{array}$ & $\begin{array}{r}0.7 \\
0\end{array}$ & $\begin{array}{r}2200 . \\
5\end{array}$ & 44.6 & 2365.5 & 31.3 & $\begin{array}{r}2511 . \\
0\end{array}$ & 41.0 & 2511.0 & 41.0 & 87.6 \\
\hline $\begin{array}{l}\text { BU0742- } \\
97\end{array}$ & 654 & $\begin{array}{r}34849 \\
5 \\
\end{array}$ & 1.3 & $\begin{array}{r}6.001 \\
3 \\
\end{array}$ & 5.3 & $\begin{array}{r}10.442 \\
0 \\
\end{array}$ & 5.6 & $\begin{array}{r}0.454 \\
5 \\
\end{array}$ & $\begin{array}{r}1 . \\
8\end{array}$ & $\begin{array}{r}0.3 \\
3 \\
\end{array}$ & $\begin{array}{r}2415 . \\
2\end{array}$ & 36.9 & 2474.8 & 52.0 & $\begin{array}{r}2524 . \\
1\end{array}$ & 89.1 & 2524.1 & 89.1 & 95.7 \\
\hline $\begin{array}{l}\text { BU0742- } \\
82\end{array}$ & 789 & $\begin{array}{r}48390 \\
5\end{array}$ & 1.2 & $\begin{array}{r}5.918 \\
6\end{array}$ & 4.1 & $\begin{array}{r}10.468 \\
1\end{array}$ & 4.5 & $\begin{array}{r}0.449 \\
4\end{array}$ & $\begin{array}{r}2 . \\
0\end{array}$ & $\begin{array}{r}0.4 \\
4 \\
\end{array}$ & $\begin{array}{r}2392 . \\
4 \\
\end{array}$ & 39.6 & 2477.1 & 42.0 & $\begin{array}{r}2547 . \\
3 \\
\end{array}$ & 68.2 & 2547.3 & 68.2 & 93.9 \\
\hline $\begin{array}{l}\text { BU0742- } \\
54\end{array}$ & 481 & 20945 & 2.6 & $\begin{array}{r}5.908 \\
2\end{array}$ & 1.8 & 8.6308 & 3.3 & $\begin{array}{r}0.369 \\
8\end{array}$ & $\begin{array}{l}2 . \\
7\end{array}$ & $\begin{array}{r}0.8 \\
3\end{array}$ & $\begin{array}{r}2028 . \\
6\end{array}$ & 47.3 & 2299.8 & 29.9 & $\begin{array}{r}2550 . \\
3\end{array}$ & 30.7 & 2550.3 & 30.7 & 79.5 \\
\hline $\begin{array}{l}\text { BU0742- } \\
11\end{array}$ & 119 & 91995 & 1.6 & $\begin{array}{r}5.576 \\
3 \\
\end{array}$ & 5.7 & $\begin{array}{r}11.836 \\
5 \\
\end{array}$ & 5.9 & $\begin{array}{r}0.478 \\
7 \\
\end{array}$ & $\begin{array}{r}1 . \\
4\end{array}$ & $\begin{array}{r}0.2 \\
4 \\
\end{array}$ & $\begin{array}{r}2521 . \\
6 \\
\end{array}$ & 29.0 & 2591.6 & 55.3 & $\begin{array}{r}2646 . \\
7 \\
\end{array}$ & 95.2 & 2646.7 & 95.2 & 95.3 \\
\hline $\begin{array}{l}\text { BU0743A- } \\
30\end{array}$ & 316 & 48080 & 0.8 & $\begin{array}{r}14.02 \\
29 \\
\end{array}$ & 4.4 & 1.5466 & 5.1 & $\begin{array}{r}0.157 \\
3 \\
\end{array}$ & $\begin{array}{r}2 . \\
5 \\
\end{array}$ & $\begin{array}{r}0.5 \\
0 \\
\end{array}$ & 941.7 & 22.3 & 949.1 & 31.3 & 966.4 & 89.9 & 941.7 & 22.3 & 97.5 \\
\hline $\begin{array}{l}\text { BU0743A- } \\
25\end{array}$ & 731 & 96055 & 2.6 & $\begin{array}{r}13.86 \\
42 \\
\end{array}$ & 1.8 & 1.6336 & 2.6 & $\begin{array}{r}0.164 \\
3 \\
\end{array}$ & $\begin{array}{r}. \\
8\end{array}$ & $\begin{array}{r}0.7 \\
1 \\
\end{array}$ & 980.4 & 16.7 & 983.2 & 16.3 & 989.5 & 36.8 & 989.5 & 36.8 & 99.1 \\
\hline $\begin{array}{l}\text { BU0743A- } \\
4\end{array}$ & 156 & 27355 & 1.4 & $\begin{array}{r}13.56 \\
49\end{array}$ & 3.0 & 1.8104 & 3.4 & $\begin{array}{r}0.178 \\
1\end{array}$ & $\begin{array}{c}1 . \\
6\end{array}$ & $\begin{array}{r}0.4 \\
7\end{array}$ & $\begin{array}{r}1056 . \\
6\end{array}$ & 15.5 & 1049.2 & 21.9 & $\begin{array}{r}1033 . \\
8\end{array}$ & 59.7 & 1033.8 & 59.7 & $\begin{array}{r}102 . \\
2\end{array}$ \\
\hline $\begin{array}{l}\text { BU0743A- } \\
31\end{array}$ & 406 & 81955 & 1.9 & $\begin{array}{r}12.87 \\
66\end{array}$ & 1.0 & 1.9159 & 3.4 & $\begin{array}{r}0.178 \\
9\end{array}$ & $\begin{array}{r}3 . \\
3\end{array}$ & $\begin{array}{r}0.9 \\
6\end{array}$ & $\begin{array}{r}1061 . \\
1\end{array}$ & 32.1 & 1086.7 & 22.9 & $\begin{array}{r}1138 . \\
2\end{array}$ & 19.9 & 1138.2 & 19.9 & 93.2 \\
\hline $\begin{array}{l}\text { BU0743A- } \\
24\end{array}$ & $\begin{array}{r}134 \\
1\end{array}$ & $\begin{array}{r}21966 \\
0\end{array}$ & 0.5 & $\begin{array}{r}12.84 \\
60\end{array}$ & 1.6 & 1.9795 & 1.9 & $\begin{array}{r}0.184 \\
4\end{array}$ & $\begin{array}{l}1 . \\
0\end{array}$ & $\begin{array}{r}0.5 \\
4\end{array}$ & $\begin{array}{r}1091 . \\
1\end{array}$ & 10.0 & 1108.6 & 12.6 & $\begin{array}{r}1142 . \\
9\end{array}$ & 31.2 & 1142.9 & 31.2 & 95.5 \\
\hline $\begin{array}{l}\text { BU0743A- } \\
9\end{array}$ & $\begin{array}{r}107 \\
5\end{array}$ & $\begin{array}{r}16710 \\
5 \\
\end{array}$ & 1.7 & $\begin{array}{r}12.73 \\
37 \\
\end{array}$ & 4.0 & 2.0682 & 4.1 & $\begin{array}{r}0.191 \\
0\end{array}$ & $\begin{array}{r}1 . \\
0\end{array}$ & $\begin{array}{r}0.2 \\
4\end{array}$ & $\begin{array}{r}1126 . \\
8\end{array}$ & 10.3 & 1138.3 & 28.2 & $\begin{array}{r}1160 . \\
3\end{array}$ & 79.1 & 1160.3 & 79.1 & 97.1 \\
\hline $\begin{array}{l}\text { BU0743A- } \\
27\end{array}$ & 609 & $\begin{array}{r}10385 \\
0 \\
\end{array}$ & 2.2 & $\begin{array}{r}12.68 \\
31\end{array}$ & 1.8 & 2.0590 & 2.0 & $\begin{array}{r}0.189 \\
4\end{array}$ & $\begin{array}{r}1 . \\
0\end{array}$ & $\begin{array}{r}0.4 \\
9\end{array}$ & $\begin{array}{r}1118 . \\
1\end{array}$ & 10.3 & 1135.3 & 13.8 & $\begin{array}{r}1168 . \\
2\end{array}$ & 34.9 & 1168.2 & 34.9 & 95.7 \\
\hline $\begin{array}{l}\text { BU0743A- } \\
17\end{array}$ & 160 & 70955 & 1.1 & $\begin{array}{r}12.42 \\
60\end{array}$ & 3.0 & 2.2602 & 3.2 & $\begin{array}{r}0.203 \\
7\end{array}$ & $\begin{array}{l}1 . \\
0\end{array}$ & $\begin{array}{r}0.3 \\
1\end{array}$ & $\begin{array}{r}1195 . \\
1\end{array}$ & 10.9 & 1200.0 & 22.5 & $\begin{array}{r}1208 . \\
7\end{array}$ & 59.7 & 1208.7 & 59.7 & 98.9 \\
\hline $\begin{array}{l}\text { BU0743A- } \\
23\end{array}$ & 844 & 61180 & 0.5 & $\begin{array}{r}12.30 \\
68\end{array}$ & 2.3 & 2.1254 & 3.5 & $\begin{array}{r}0.189 \\
7\end{array}$ & $\begin{array}{r}2 . \\
6\end{array}$ & $\begin{array}{r}0.7 \\
5\end{array}$ & $\begin{array}{r}1119 . \\
8\end{array}$ & 27.0 & 1157.1 & 24.2 & $\begin{array}{r}1227 . \\
6\end{array}$ & 45.4 & 1227.6 & 45.4 & 91.2 \\
\hline $\begin{array}{l}\text { BU0743A- } \\
12\end{array}$ & 183 & 59460 & 1.2 & $\begin{array}{r}12.29 \\
09\end{array}$ & 1.7 & 2.4233 & 2.0 & $\begin{array}{r}0.216 \\
0\end{array}$ & $\begin{array}{l}1 . \\
0\end{array}$ & $\begin{array}{r}0.5 \\
0\end{array}$ & $\begin{array}{r}1260 . \\
8\end{array}$ & 11.5 & 1249.5 & 14.4 & $\begin{array}{r}1230 . \\
2\end{array}$ & 34.2 & 1230.2 & 34.2 & $\begin{array}{r}102 . \\
5\end{array}$ \\
\hline $\begin{array}{l}\text { BU0743A- } \\
26\end{array}$ & 344 & 70305 & 4.3 & $\begin{array}{r}12.23 \\
14\end{array}$ & 1.3 & 2.1627 & 1.7 & $\begin{array}{r}0.191 \\
9\end{array}$ & $\begin{array}{r}1 . \\
0\end{array}$ & $\begin{array}{r}0.6 \\
0\end{array}$ & $\begin{array}{r}1131 . \\
4\end{array}$ & 10.4 & 1169.1 & 11.6 & $\begin{array}{r}1239 . \\
7\end{array}$ & 26.1 & 1239.7 & 26.1 & 91.3 \\
\hline $\begin{array}{l}\text { BU0743A- } \\
22\end{array}$ & $\begin{array}{r}233 \\
9 \\
\end{array}$ & 94450 & 1.7 & $\begin{array}{r}12.17 \\
50\end{array}$ & 1.1 & 1.9314 & 1.8 & $\begin{array}{r}0.170 \\
5\end{array}$ & $\begin{array}{r}1 . \\
4\end{array}$ & $\begin{array}{r}0.7 \\
8\end{array}$ & $\begin{array}{r}1015 . \\
1\end{array}$ & 12.9 & 1092.0 & 11.8 & $\begin{array}{r}1248 . \\
7\end{array}$ & 21.5 & 1248.7 & 21.5 & 81.3 \\
\hline $\begin{array}{l}\text { BU0743A- } \\
13\end{array}$ & 980 & 72840 & 0.4 & $\begin{array}{r}11.60 \\
84\end{array}$ & 3.7 & 2.4702 & 4.6 & $\begin{array}{r}0.208 \\
0\end{array}$ & $\begin{array}{r}2 . \\
9\end{array}$ & $\begin{array}{r}0.6 \\
2\end{array}$ & $\begin{array}{r}1218 . \\
0\end{array}$ & 31.7 & 1263.3 & 33.5 & $\begin{array}{r}1341 . \\
4\end{array}$ & 70.5 & 1341.4 & 70.5 & 90.8 \\
\hline $\begin{array}{l}\text { BU0743A- } \\
33\end{array}$ & 405 & 97655 & 1.4 & $\begin{array}{r}11.54 \\
45\end{array}$ & 1.6 & 2.7068 & 2.9 & $\begin{array}{r}0.226 \\
6\end{array}$ & $\begin{array}{r}2 . \\
5\end{array}$ & $\begin{array}{r}0.8 \\
5\end{array}$ & $\begin{array}{r}1316 . \\
8\end{array}$ & 29.8 & 1330.3 & 21.9 & $\begin{array}{r}1352 . \\
1\end{array}$ & 30.1 & 1352.1 & 30.1 & 97.4 \\
\hline $\begin{array}{l}\text { BU0743A- } \\
11\end{array}$ & 578 & $\begin{array}{r}19354 \\
5\end{array}$ & 1.1 & $\begin{array}{r}11.44 \\
30\end{array}$ & 3.3 & 2.8720 & 4.3 & $\begin{array}{r}0.238 \\
4\end{array}$ & $\begin{array}{r}2 . \\
7\end{array}$ & $\begin{array}{r}0.6 \\
3\end{array}$ & $\begin{array}{r}1378 . \\
1\end{array}$ & 33.1 & 1374.6 & 32.1 & $\begin{array}{r}1369 . \\
1\end{array}$ & 63.9 & 1369.1 & 63.9 & $\begin{array}{r}100 . \\
7\end{array}$ \\
\hline
\end{tabular}




\begin{tabular}{|c|c|c|c|c|c|c|c|c|c|c|c|c|c|c|c|c|c|c|c|}
\hline $\begin{array}{l}\text { BU0743A- } \\
28\end{array}$ & 609 & $\begin{array}{r}11802 \\
0 \\
\end{array}$ & 1.2 & $\begin{array}{r}11.38 \\
12 \\
\end{array}$ & 3.2 & 2.6257 & 4.1 & $\begin{array}{r}0.216 \\
7 \\
\end{array}$ & $\begin{array}{r}2 . \\
5 \\
\end{array}$ & $\begin{array}{r}0.6 \\
2 \\
\end{array}$ & $\begin{array}{r}1264 . \\
6 \\
\end{array}$ & 28.8 & 1307.9 & 29.9 & $\begin{array}{r}1379 . \\
5 \\
\end{array}$ & 61.5 & 1379.5 & 61.5 & 91.7 \\
\hline $\begin{array}{l}\text { BU0743A- } \\
10\end{array}$ & 541 & 96410 & 1.4 & $\begin{array}{r}11.32 \\
10 \\
\end{array}$ & 2.0 & 2.9090 & 2.3 & $\begin{array}{r}0.238 \\
9 \\
\end{array}$ & $\begin{array}{c}1 . \\
1\end{array}$ & $\begin{array}{r}0.4 \\
6 \\
\end{array}$ & $\begin{array}{r}1380 . \\
7 \\
\end{array}$ & 13.2 & 1384.3 & 17.3 & $\begin{array}{r}1389 . \\
7 \\
\end{array}$ & 39.0 & 1389.7 & 39.0 & 99.4 \\
\hline $\begin{array}{l}\text { BU0743A- } \\
3\end{array}$ & 322 & $\begin{array}{r}13743 \\
0 \\
\end{array}$ & 1.2 & $\begin{array}{r}10.99 \\
55 \\
\end{array}$ & 3.2 & 3.2333 & 3.3 & $\begin{array}{r}0.257 \\
8 \\
\end{array}$ & $\begin{array}{r}1 . \\
0\end{array}$ & $\begin{array}{r}0.3 \\
0 \\
\end{array}$ & $\begin{array}{r}1478 . \\
8 \\
\end{array}$ & 13.2 & 1465.2 & 25.9 & $\begin{array}{r}1445 . \\
5 \\
\end{array}$ & 60.6 & 1445.5 & 60.6 & $\begin{array}{r}102 . \\
3 \\
\end{array}$ \\
\hline $\begin{array}{l}\text { BU0743A- } \\
6\end{array}$ & 330 & $\begin{array}{r}11463 \\
0 \\
\end{array}$ & 1.2 & $\begin{array}{r}10.55 \\
47 \\
\end{array}$ & 1.3 & 3.4657 & 2.0 & $\begin{array}{r}0.265 \\
3 \\
\end{array}$ & $\begin{array}{c}1 . \\
5\end{array}$ & $\begin{array}{r}0.7 \\
6 \\
\end{array}$ & $\begin{array}{r}1516 . \\
9 \\
\end{array}$ & 20.5 & 1519.5 & 15.7 & $\begin{array}{r}1523 . \\
0 \\
\end{array}$ & 24.1 & 1523.0 & 24.1 & 99.6 \\
\hline $\begin{array}{l}\text { BU0743A- } \\
21\end{array}$ & 265 & 59400 & 1.5 & $\begin{array}{r}10.19 \\
25 \\
\end{array}$ & 1.6 & 3.7028 & 3.1 & $\begin{array}{r}0.273 \\
7 \\
\end{array}$ & $\begin{array}{r}2 . \\
6 \\
\end{array}$ & $\begin{array}{r}0.8 \\
5 \\
\end{array}$ & $\begin{array}{r}1559 . \\
7 \\
\end{array}$ & 36.2 & 1572.0 & 24.6 & $\begin{array}{r}1588 . \\
5 \\
\end{array}$ & 30.6 & 1588.5 & 30.6 & 98.2 \\
\hline $\begin{array}{l}\text { BU0743A- } \\
32\end{array}$ & 323 & 62955 & 1.0 & $\begin{array}{r}10.12 \\
96 \\
\end{array}$ & 1.9 & 3.7954 & 2.3 & $\begin{array}{r}0.278 \\
8 \\
\end{array}$ & $\begin{array}{r}1 . \\
2 \\
\end{array}$ & $\begin{array}{r}0.5 \\
3 \\
\end{array}$ & $\begin{array}{r}1585 . \\
5 \\
\end{array}$ & 17.0 & 1591.8 & 18.2 & $\begin{array}{r}1600 . \\
1 \\
\end{array}$ & 35.8 & 1600.1 & 35.8 & 99.1 \\
\hline $\begin{array}{l}\text { BU0743A- } \\
16\end{array}$ & 476 & 88610 & 0.9 & $\begin{array}{r}9.861 \\
3 \\
\end{array}$ & 3.1 & 4.1806 & 3.2 & $\begin{array}{r}0.299 \\
0 \\
\end{array}$ & $\begin{array}{l}1 . \\
0\end{array}$ & $\begin{array}{r}0.3 \\
1 \\
\end{array}$ & $\begin{array}{r}1686 . \\
4 \\
\end{array}$ & 14.8 & 1670.2 & 26.3 & $\begin{array}{r}1650 . \\
0 \\
\end{array}$ & 56.6 & 1650.0 & 56.6 & $\begin{array}{r}102 . \\
2 \\
\end{array}$ \\
\hline $\begin{array}{l}\text { BU0743A- } \\
1\end{array}$ & 271 & $\begin{array}{r}10465 \\
0 \\
\end{array}$ & 6.9 & $\begin{array}{r}9.569 \\
8 \\
\end{array}$ & 2.5 & 4.4429 & 2.7 & $\begin{array}{r}0.308 \\
4 \\
\end{array}$ & $\begin{array}{l}1 . \\
0\end{array}$ & $\begin{array}{r}0.3 \\
7 \\
\end{array}$ & $\begin{array}{r}1732 . \\
7 \\
\end{array}$ & 15.2 & 1720.4 & 22.4 & $\begin{array}{r}1705 . \\
4 \\
\end{array}$ & 46.2 & 1705.4 & 46.2 & $\begin{array}{r}101 . \\
6 \\
\end{array}$ \\
\hline $\begin{array}{l}\text { BU0743A- } \\
15\end{array}$ & 588 & 35165 & 1.9 & $\begin{array}{r}9.461 \\
7 \\
\end{array}$ & 2.6 & 4.2966 & 2.8 & $\begin{array}{r}0.294 \\
8 \\
\end{array}$ & $\begin{array}{r}1 . \\
0\end{array}$ & $\begin{array}{r}0.3 \\
6 \\
\end{array}$ & $\begin{array}{r}1665 . \\
7 \\
\end{array}$ & 14.7 & 1692.7 & 22.9 & $\begin{array}{r}1726 . \\
3 \\
\end{array}$ & 47.6 & 1726.3 & 47.6 & 96.5 \\
\hline $\begin{array}{l}\text { BU0743A- } \\
29\end{array}$ & 269 & 55810 & 1.7 & $\begin{array}{r}9.457 \\
0 \\
\end{array}$ & 2.0 & 3.6990 & 2.6 & $\begin{array}{r}0.253 \\
7 \\
\end{array}$ & $\begin{array}{l}1 . \\
7 \\
\end{array}$ & $\begin{array}{r}0.6 \\
3 \\
\end{array}$ & $\begin{array}{r}1457 . \\
6 \\
\end{array}$ & 21.5 & 1571.2 & 20.9 & $\begin{array}{r}1727 . \\
2 \\
\end{array}$ & 37.1 & 1727.2 & 37.1 & 84.4 \\
\hline $\begin{array}{l}\text { BU0743A- } \\
5\end{array}$ & 174 & 54360 & 1.0 & $\begin{array}{r}9.341 \\
1 \\
\end{array}$ & 4.7 & 4.7831 & 5.6 & $\begin{array}{r}0.324 \\
0 \\
\end{array}$ & $\begin{array}{r}3 . \\
1\end{array}$ & $\begin{array}{r}0.5 \\
5 \\
\end{array}$ & $\begin{array}{r}1809 . \\
4 \\
\end{array}$ & 48.3 & 1781.9 & 47.1 & $\begin{array}{r}1749 . \\
8 \\
\end{array}$ & 85.9 & 1749.8 & 85.9 & $\begin{array}{r}103 . \\
4 \\
\end{array}$ \\
\hline $\begin{array}{l}\text { BU0743A- } \\
14\end{array}$ & 161 & 85045 & 1.3 & $\begin{array}{r}8.827 \\
2 \\
\end{array}$ & 2.6 & 5.2847 & 3.4 & $\begin{array}{r}0.338 \\
3 \\
\end{array}$ & $\begin{array}{r}2 . \\
2 \\
\end{array}$ & $\begin{array}{r}0.6 \\
5 \\
\end{array}$ & $\begin{array}{r}1878 . \\
6 \\
\end{array}$ & 36.0 & 1866.4 & 28.9 & $\begin{array}{r}1852 . \\
8 \\
\end{array}$ & 46.5 & 1852.8 & 46.5 & $\begin{array}{r}101 . \\
4 \\
\end{array}$ \\
\hline $\begin{array}{l}\text { BU0743A- } \\
19\end{array}$ & 871 & 56145 & 1.2 & $\begin{array}{r}8.266 \\
9 \\
\end{array}$ & 2.5 & 5.4500 & 2.7 & $\begin{array}{r}0.326 \\
8 \\
\end{array}$ & $\begin{array}{r}1 . \\
0\end{array}$ & $\begin{array}{r}0.3 \\
7 \\
\end{array}$ & $\begin{array}{r}1822 . \\
7 \\
\end{array}$ & 15.9 & 1892.7 & 23.3 & $\begin{array}{r}1970 . \\
5 \\
\end{array}$ & 44.9 & 1970.5 & 44.9 & 92.5 \\
\hline $\begin{array}{l}\text { BU0743A- } \\
20\end{array}$ & 193 & 18930 & 1.7 & $\begin{array}{r}7.207 \\
7 \\
\end{array}$ & 2.2 & 6.4637 & 5.8 & $\begin{array}{r}0.337 \\
9 \\
\end{array}$ & $\begin{array}{r}5 . \\
4 \\
\end{array}$ & $\begin{array}{r}0.9 \\
3 \\
\end{array}$ & $\begin{array}{r}1876 . \\
5 \\
\end{array}$ & 87.9 & 2041.0 & 51.3 & $\begin{array}{r}2211 . \\
5 \\
\end{array}$ & 37.9 & 2211.5 & 37.9 & 84.9 \\
\hline $\begin{array}{l}\text { BU0743A- } \\
2\end{array}$ & 577 & 38430 & 2.1 & $\begin{array}{r}6.208 \\
8 \\
\end{array}$ & 2.4 & 6.8404 & 3.4 & $\begin{array}{r}0.308 \\
0 \\
\end{array}$ & $\begin{array}{r}2 . \\
4\end{array}$ & $\begin{array}{r}0.7 \\
0 \\
\end{array}$ & $\begin{array}{r}1731 . \\
0 \\
\end{array}$ & 36.1 & 2091.0 & 30.1 & $\begin{array}{r}2466 . \\
8 \\
\end{array}$ & 41.1 & 2466.8 & 41.1 & 70.2 \\
\hline $\begin{array}{l}\text { BU0743A- } \\
18\end{array}$ & $\begin{array}{r}119 \\
3 \\
\end{array}$ & $\begin{array}{r}47042 \\
0 \\
\end{array}$ & 2.7 & $\begin{array}{r}5.928 \\
8 \\
\end{array}$ & 1.9 & $\begin{array}{r}11.358 \\
3 \\
\end{array}$ & 2.2 & $\begin{array}{r}0.488 \\
4 \\
\end{array}$ & $\begin{array}{r}1 . \\
0\end{array}$ & $\begin{array}{r}0.4 \\
6 \\
\end{array}$ & $\begin{array}{r}2563 . \\
7 \\
\end{array}$ & 21.2 & 2553.0 & 20.2 & $\begin{array}{r}2544 . \\
5 \\
\end{array}$ & 32.2 & 2544.5 & 32.2 & $\begin{array}{r}100 . \\
8 \\
\end{array}$ \\
\hline $\begin{array}{l}\text { BU0753- } \\
44\end{array}$ & 505 & 63225 & 2.2 & $\begin{array}{r}18.40 \\
42 \\
\end{array}$ & 1.8 & 0.4765 & 2.9 & $\begin{array}{r}0.063 \\
6 \\
\end{array}$ & $\begin{array}{r}2 . \\
2\end{array}$ & $\begin{array}{r}0.7 \\
8 \\
\end{array}$ & 397.5 & 8.6 & 395.7 & 9.4 & 385.0 & 40.1 & 397.5 & 8.6 & NA \\
\hline $\begin{array}{l}\text { BU0753- } \\
90\end{array}$ & 554 & 49995 & 1.6 & $\begin{array}{r}18.06 \\
75 \\
\end{array}$ & 1.6 & 0.5161 & 3.6 & $\begin{array}{r}0.067 \\
6 \\
\end{array}$ & $\begin{array}{r}3 . \\
2 \\
\end{array}$ & $\begin{array}{r}0.8 \\
9 \\
\end{array}$ & 421.8 & 13.1 & 422.5 & 12.4 & 426.3 & 36.4 & 421.8 & 13.1 & NA \\
\hline $\begin{array}{l}\text { BU0753- } \\
54\end{array}$ & 163 & 23480 & 1.3 & $\begin{array}{r}17.72 \\
91 \\
\end{array}$ & 2.5 & 0.5960 & 3.7 & $\begin{array}{r}0.076 \\
6 \\
\end{array}$ & $\begin{array}{r}2 . \\
8 \\
\end{array}$ & $\begin{array}{r}0.7 \\
4 \\
\end{array}$ & 476.0 & 12.7 & 474.7 & 14.2 & 468.3 & 55.8 & 476.0 & 12.7 & NA \\
\hline $\begin{array}{l}\text { BU0753- } \\
68\end{array}$ & 303 & 24420 & 0.8 & $\begin{array}{r}17.00 \\
74 \\
\end{array}$ & 6.8 & 0.6284 & 7.6 & $\begin{array}{r}0.077 \\
5 \\
\end{array}$ & $\begin{array}{c}3 . \\
5\end{array}$ & $\begin{array}{r}0.4 \\
6 \\
\end{array}$ & 481.3 & 16.4 & 495.1 & 29.9 & 559.6 & $\begin{array}{r}147 . \\
5 \\
\end{array}$ & 481.3 & 16.4 & NA \\
\hline $\begin{array}{l}\text { BU0753- } \\
82\end{array}$ & 123 & 15045 & 1.9 & $\begin{array}{r}18.34 \\
52 \\
\end{array}$ & 5.7 & 0.5874 & 6.1 & $\begin{array}{r}0.078 \\
2 \\
\end{array}$ & $\begin{array}{r}2 . \\
0 \\
\end{array}$ & $\begin{array}{r}0.3 \\
3 \\
\end{array}$ & 485.1 & 9.5 & 469.2 & 22.9 & 392.2 & $\begin{array}{r}128 . \\
7 \\
\end{array}$ & 485.1 & 9.5 & NA \\
\hline $\begin{array}{l}\text { BU0753- } \\
81\end{array}$ & 376 & 59285 & 1.6 & $\begin{array}{r}17.63 \\
63 \\
\end{array}$ & 1.7 & 0.6156 & 2.6 & $\begin{array}{r}0.078 \\
7 \\
\end{array}$ & $\begin{array}{r}2 . \\
0 \\
\end{array}$ & $\begin{array}{r}0.7 \\
7 \\
\end{array}$ & 488.6 & 9.5 & 487.1 & 10.2 & 479.9 & 37.4 & 488.6 & 9.5 & NA \\
\hline BU0753-1 & 792 & 91960 & 2.2 & $\begin{array}{r}17.61 \\
54 \\
\end{array}$ & 1.0 & 0.6180 & 1.6 & $\begin{array}{r}0.079 \\
0 \\
\end{array}$ & $\begin{array}{r}1 . \\
2\end{array}$ & $\begin{array}{r}0.7 \\
7 \\
\end{array}$ & 489.9 & 5.7 & 488.6 & 6.1 & 482.5 & 22.1 & 489.9 & 5.7 & NA \\
\hline $\begin{array}{l}\text { BU0753- } \\
62\end{array}$ & 267 & 23920 & 0.9 & $\begin{array}{r}17.82 \\
93 \\
\end{array}$ & 2.7 & 0.6115 & 3.3 & $\begin{array}{r}0.079 \\
1 \\
\end{array}$ & $\begin{array}{r}1 . \\
8 \\
\end{array}$ & $\begin{array}{r}0.5 \\
5 \\
\end{array}$ & 490.6 & 8.5 & 484.5 & 12.5 & 455.8 & 60.3 & 490.6 & 8.5 & NA \\
\hline
\end{tabular}




\begin{tabular}{|c|c|c|c|c|c|c|c|c|c|c|c|c|c|c|c|c|c|c|c|}
\hline $\begin{array}{l}\text { BU0753- } \\
83\end{array}$ & 289 & 32640 & 0.9 & $\begin{array}{r}17.84 \\
63 \\
\end{array}$ & 2.5 & 0.6143 & 2.9 & $\begin{array}{r}0.079 \\
5 \\
\end{array}$ & $\begin{array}{r}1 . \\
5\end{array}$ & $\begin{array}{r}0.5 \\
1 \\
\end{array}$ & 493.2 & 7.0 & 486.3 & 11.2 & 453.7 & 55.6 & 493.2 & 7.0 & NA \\
\hline $\begin{array}{l}\text { BU0753- } \\
73\end{array}$ & 321 & 50895 & 1.0 & $\begin{array}{r}17.52 \\
96\end{array}$ & 4.0 & 0.6263 & 4.5 & $\begin{array}{r}0.079 \\
6\end{array}$ & $\begin{array}{r}2 . \\
2\end{array}$ & $\begin{array}{r}0.4 \\
8\end{array}$ & 493.9 & 10.3 & 493.8 & 17.7 & 493.3 & 88.0 & 493.9 & 10.3 & NA \\
\hline $\begin{array}{l}\text { BU0753- } \\
25\end{array}$ & 299 & 36155 & 0.8 & $\begin{array}{r}17.22 \\
06\end{array}$ & 2.4 & 0.6388 & 3.4 & $\begin{array}{r}0.079 \\
8\end{array}$ & $\begin{array}{r}2 . \\
4\end{array}$ & $\begin{array}{r}0.6 \\
9\end{array}$ & 494.8 & 11.2 & 501.6 & 13.4 & 532.4 & 53.3 & 494.8 & 11.2 & NA \\
\hline $\begin{array}{l}\text { BU0753- } \\
79\end{array}$ & 90 & 20030 & 0.8 & $\begin{array}{r}17.60 \\
37 \\
\end{array}$ & 6.8 & 0.6253 & 7.4 & $\begin{array}{r}0.079 \\
8 \\
\end{array}$ & $\begin{array}{r}2 . \\
8\end{array}$ & $\begin{array}{r}0.3 \\
8 \\
\end{array}$ & 495.1 & 13.2 & 493.1 & 28.8 & 484.0 & $\begin{array}{r}150 . \\
8\end{array}$ & 495.1 & 13.2 & NA \\
\hline $\begin{array}{l}\text { BU0753- } \\
49\end{array}$ & 214 & 33315 & 0.8 & $\begin{array}{r}17.47 \\
34 \\
\end{array}$ & 3.6 & 0.6364 & 3.8 & $\begin{array}{r}0.080 \\
7 \\
\end{array}$ & $\begin{array}{r}1 . \\
0\end{array}$ & $\begin{array}{r}0.2 \\
6 \\
\end{array}$ & 500.0 & 4.8 & 500.1 & 14.9 & 500.4 & 80.3 & 500.0 & 4.8 & 99.9 \\
\hline $\begin{array}{l}\text { BU0753- } \\
77\end{array}$ & 289 & 36575 & 1.9 & $\begin{array}{r}17.88 \\
70 \\
\end{array}$ & 3.8 & 0.6227 & 4.8 & $\begin{array}{r}0.080 \\
8 \\
\end{array}$ & $\begin{array}{r}3 . \\
0\end{array}$ & $\begin{array}{r}0.6 \\
2 \\
\end{array}$ & 500.8 & 14.5 & 491.5 & 18.7 & 448.6 & 83.3 & 500.8 & 14.5 & $\begin{array}{r}111 . \\
6 \\
\end{array}$ \\
\hline $\begin{array}{l}\text { BU0753- } \\
51\end{array}$ & 100 & 16000 & 0.9 & $\begin{array}{r}18.22 \\
16\end{array}$ & 5.5 & 0.6125 & 5.6 & $\begin{array}{r}0.080 \\
9\end{array}$ & $\begin{array}{r}1 . \\
3\end{array}$ & $\begin{array}{r}0.2 \\
3\end{array}$ & 501.7 & 6.2 & 485.1 & 21.8 & 407.3 & $\begin{array}{r}123 . \\
2\end{array}$ & 501.7 & 6.2 & $\begin{array}{r}123 . \\
2\end{array}$ \\
\hline $\begin{array}{l}\text { BU0753- } \\
59\end{array}$ & 810 & $\begin{array}{r}11256 \\
0 \\
\end{array}$ & 1.7 & $\begin{array}{r}17.39 \\
26 \\
\end{array}$ & 1.6 & 0.6445 & 2.0 & $\begin{array}{r}0.081 \\
3 \\
\end{array}$ & $\begin{array}{r}1 . \\
3\end{array}$ & $\begin{array}{r}0.6 \\
3 \\
\end{array}$ & 503.9 & 6.2 & 505.1 & 8.0 & 510.6 & 34.3 & 503.9 & 6.2 & 98.7 \\
\hline $\begin{array}{l}\text { BU0753- } \\
75\end{array}$ & 325 & 51410 & 1.4 & $\begin{array}{r}17.83 \\
66 \\
\end{array}$ & 3.2 & 0.6332 & 4.1 & $\begin{array}{r}0.081 \\
9 \\
\end{array}$ & $\begin{array}{r}2 . \\
6 \\
\end{array}$ & $\begin{array}{r}0.6 \\
3 \\
\end{array}$ & 507.5 & 12.5 & 498.1 & 16.0 & 454.9 & 69.9 & 507.5 & 12.5 & $\begin{array}{r}111 . \\
6 \\
\end{array}$ \\
\hline $\begin{array}{l}\text { BU0753- } \\
20\end{array}$ & 253 & 34775 & 0.8 & $\begin{array}{r}17.32 \\
39 \\
\end{array}$ & 1.4 & 0.6522 & 2.7 & $\begin{array}{r}0.081 \\
9 \\
\end{array}$ & $\begin{array}{r}2 . \\
3 \\
\end{array}$ & $\begin{array}{r}0.8 \\
5 \\
\end{array}$ & 507.7 & 11.3 & 509.8 & 10.8 & 519.3 & 30.8 & 507.7 & 11.3 & 97.8 \\
\hline $\begin{array}{l}\text { BU0753- } \\
60\end{array}$ & 153 & 27945 & 1.2 & $\begin{array}{r}17.76 \\
26\end{array}$ & 2.6 & 0.6375 & 2.8 & $\begin{array}{r}0.082 \\
1\end{array}$ & $\begin{array}{r}1 . \\
2\end{array}$ & $\begin{array}{r}0.4 \\
1\end{array}$ & 508.8 & 5.7 & 500.8 & 11.1 & 464.1 & 56.8 & 508.8 & 5.7 & $\begin{array}{r}109 . \\
6\end{array}$ \\
\hline $\begin{array}{l}\text { BU0753- } \\
98\end{array}$ & 117 & 9135 & 0.5 & $\begin{array}{r}17.01 \\
85\end{array}$ & 3.1 & 0.6685 & 4.6 & $\begin{array}{r}0.082 \\
5\end{array}$ & $\begin{array}{r}3 . \\
4\end{array}$ & $\begin{array}{r}0.7 \\
4\end{array}$ & 511.1 & 16.9 & 519.8 & 18.8 & 558.2 & 67.8 & 511.1 & 16.9 & 91.6 \\
\hline $\begin{array}{l}\text { BU0753- } \\
31\end{array}$ & 317 & 31295 & 1.0 & $\begin{array}{r}17.04 \\
28 \\
\end{array}$ & 1.2 & 0.6749 & 4.9 & $\begin{array}{r}0.083 \\
4 \\
\end{array}$ & $\begin{array}{r}4 . \\
8 \\
\end{array}$ & $\begin{array}{r}0.9 \\
7 \\
\end{array}$ & 516.5 & 23.6 & 523.7 & 20.1 & 555.1 & 27.1 & 516.5 & 23.6 & 93.1 \\
\hline BU0753-6 & 206 & 97070 & 0.7 & $\begin{array}{r}16.60 \\
76 \\
\end{array}$ & 4.8 & 0.6931 & 4.9 & $\begin{array}{r}0.083 \\
5 \\
\end{array}$ & $\begin{array}{r}1 . \\
0\end{array}$ & $\begin{array}{r}0.2 \\
0 \\
\end{array}$ & 516.9 & 5.0 & 534.6 & 20.4 & 611.2 & $\begin{array}{r}104 . \\
0 \\
\end{array}$ & 516.9 & 5.0 & 84.6 \\
\hline $\begin{array}{l}\text { BU0753- } \\
47\end{array}$ & 215 & 48745 & 2.0 & $\begin{array}{r}16.67 \\
22 \\
\end{array}$ & 4.4 & 0.7009 & 4.6 & $\begin{array}{r}0.084 \\
8 \\
\end{array}$ & $\begin{array}{r}1 . \\
4\end{array}$ & $\begin{array}{r}0.3 \\
1 \\
\end{array}$ & 524.4 & 7.2 & 539.3 & 19.2 & 602.8 & 94.6 & 524.4 & 7.2 & 87.0 \\
\hline $\begin{array}{l}\text { BU0753- } \\
32\end{array}$ & 199 & 34850 & 1.2 & $\begin{array}{r}17.01 \\
79\end{array}$ & 3.4 & 0.6872 & 5.3 & $\begin{array}{r}0.084 \\
8\end{array}$ & $\begin{array}{r}4 . \\
1\end{array}$ & $\begin{array}{r}0.7 \\
7\end{array}$ & 524.8 & 20.5 & 531.1 & 21.9 & 558.3 & 74.3 & 524.8 & 20.5 & 94.0 \\
\hline $\begin{array}{l}\text { BU0753- } \\
26\end{array}$ & 899 & $\begin{array}{r}18272 \\
0 \\
\end{array}$ & 3.9 & $\begin{array}{r}17.14 \\
04 \\
\end{array}$ & 1.3 & 0.6843 & 1.9 & $\begin{array}{r}0.085 \\
1 \\
\end{array}$ & $\begin{array}{r}1 . \\
3\end{array}$ & $\begin{array}{r}0.7 \\
2 \\
\end{array}$ & 526.3 & 6.7 & 529.4 & 7.6 & 542.6 & 28.2 & 526.3 & 6.7 & 97.0 \\
\hline $\begin{array}{l}\text { BU0753- } \\
88\end{array}$ & 114 & 13080 & 1.4 & $\begin{array}{r}17.39 \\
79 \\
\end{array}$ & 2.6 & 0.6749 & 5.4 & $\begin{array}{r}0.085 \\
2 \\
\end{array}$ & $\begin{array}{r}4 . \\
8 \\
\end{array}$ & $\begin{array}{r}0.8 \\
8 \\
\end{array}$ & 526.9 & 24.1 & 523.7 & 22.2 & 509.9 & 57.4 & 526.9 & 24.1 & $\begin{array}{r}103 . \\
3 \\
\end{array}$ \\
\hline $\begin{array}{l}\text { BU0753- } \\
71\end{array}$ & 250 & 50860 & 0.9 & $\begin{array}{r}17.26 \\
22 \\
\end{array}$ & 3.0 & 0.6826 & 3.2 & $\begin{array}{r}0.085 \\
5 \\
\end{array}$ & $\begin{array}{r}1 . \\
3 \\
\end{array}$ & $\begin{array}{r}0.4 \\
1 \\
\end{array}$ & 528.6 & 6.6 & 528.4 & 13.3 & 527.1 & 64.7 & 528.6 & 6.6 & $\begin{array}{r}100 . \\
3 \\
\end{array}$ \\
\hline BU0753-7 & 213 & 36680 & 0.8 & $\begin{array}{r}17.66 \\
15\end{array}$ & 5.4 & 0.6711 & 5.5 & $\begin{array}{r}0.086 \\
0\end{array}$ & $\begin{array}{r}1 . \\
0\end{array}$ & $\begin{array}{r}0.1 \\
8\end{array}$ & 531.6 & 5.1 & 521.4 & 22.3 & 476.8 & $\begin{array}{r}118 . \\
8\end{array}$ & 531.6 & 5.1 & $\begin{array}{r}111 . \\
5\end{array}$ \\
\hline $\begin{array}{l}\text { BU0753- } \\
92\end{array}$ & 412 & 40505 & 2.6 & $\begin{array}{r}17.15 \\
06\end{array}$ & 1.4 & 0.6925 & 2.3 & $\begin{array}{r}0.086 \\
1\end{array}$ & $\begin{array}{r}1 . \\
8\end{array}$ & $\begin{array}{r}0.7 \\
8\end{array}$ & 532.6 & 9.1 & 534.3 & 9.4 & 541.3 & 30.9 & 532.6 & 9.1 & 98.4 \\
\hline $\begin{array}{l}\text { BU0753- } \\
76\end{array}$ & 171 & 58300 & 1.7 & $\begin{array}{r}16.60 \\
78\end{array}$ & 3.2 & 0.7877 & 3.5 & $\begin{array}{r}0.094 \\
9\end{array}$ & $\begin{array}{r}1 . \\
4\end{array}$ & $\begin{array}{r}0.4 \\
0\end{array}$ & 584.3 & 7.7 & 589.9 & 15.6 & 611.2 & 69.0 & 584.3 & 7.7 & 95.6 \\
\hline $\begin{array}{l}\text { BU0753- } \\
17\end{array}$ & 738 & 97640 & 2.2 & $\begin{array}{r}14.92 \\
17\end{array}$ & 3.4 & 0.9195 & 5.8 & $\begin{array}{r}0.099 \\
5\end{array}$ & $\begin{array}{r}4 . \\
7\end{array}$ & $\begin{array}{r}0.8 \\
1\end{array}$ & 611.5 & 27.1 & 662.1 & 28.1 & 838.3 & 71.0 & 611.5 & 27.1 & 73.0 \\
\hline $\begin{array}{l}\text { BU0753- } \\
97\end{array}$ & 858 & 88835 & 5.2 & $\begin{array}{r}15.18 \\
00 \\
\end{array}$ & 2.9 & 1.0177 & 3.0 & $\begin{array}{r}0.112 \\
0 \\
\end{array}$ & $\begin{array}{r}1 . \\
0\end{array}$ & $\begin{array}{r}0.3 \\
3 \\
\end{array}$ & 684.6 & 6.5 & 712.8 & 15.5 & 802.4 & 59.9 & 684.6 & 6.5 & 85.3 \\
\hline BU0753-9 & 192 & 96605 & 2.3 & 15.53 & 5.6 & 1.0138 & 5.7 & 0.114 & 1. & 0.1 & 697.3 & 6.6 & 710.8 & 29.2 & 753.7 & 118. & 697.3 & 6.6 & 92.5 \\
\hline
\end{tabular}




\begin{tabular}{|c|c|c|c|c|c|c|c|c|c|c|c|c|c|c|c|c|c|c|c|}
\hline & & & & 57 & & & & 2 & 0 & 8 & & & & & & 7 & & & \\
\hline $\begin{array}{l}\text { BU0753- } \\
95\end{array}$ & 177 & 20035 & 1.0 & $\begin{array}{r}15.08 \\
81\end{array}$ & 3.0 & 1.1194 & 3.1 & $\begin{array}{r}0.122 \\
5\end{array}$ & $\begin{array}{r}1 . \\
0\end{array}$ & $\begin{array}{r}0.3 \\
2\end{array}$ & 744.9 & 7.0 & 762.7 & 16.8 & 815.1 & 62.0 & 744.9 & 7.0 & 91.4 \\
\hline $\begin{array}{l}\text { BU0753- } \\
63\end{array}$ & 206 & 26830 & 0.4 & $\begin{array}{r}14.78 \\
23\end{array}$ & 1.1 & 1.2276 & 1.5 & $\begin{array}{r}0.131 \\
6\end{array}$ & $\begin{array}{r}1 . \\
0\end{array}$ & $\begin{array}{r}0.6 \\
6\end{array}$ & 797.1 & 7.5 & 813.3 & 8.5 & 857.8 & 23.5 & 797.1 & 7.5 & 92.9 \\
\hline $\begin{array}{l}\text { BU0753- } \\
21\end{array}$ & 249 & 48290 & 1.8 & $\begin{array}{r}15.05 \\
19\end{array}$ & 2.0 & 1.2073 & 2.5 & $\begin{array}{r}0.131 \\
8\end{array}$ & $\begin{array}{r}1 . \\
5\end{array}$ & $\begin{array}{r}0.5 \\
9\end{array}$ & 798.1 & 11.2 & 804.0 & 14.0 & 820.1 & 42.6 & 798.1 & 11.2 & 97.3 \\
\hline $\begin{array}{l}\text { BU0753- } \\
24\end{array}$ & 175 & 29120 & 0.5 & $\begin{array}{r}15.26 \\
61\end{array}$ & 1.9 & 1.2007 & 3.0 & $\begin{array}{r}0.132 \\
9\end{array}$ & $\begin{array}{r}2 . \\
3\end{array}$ & $\begin{array}{r}0.7 \\
7\end{array}$ & 804.7 & 17.7 & 800.9 & 16.8 & 790.6 & 40.4 & 804.7 & 17.7 & $\begin{array}{r}101 . \\
8\end{array}$ \\
\hline $\begin{array}{l}\text { BU0753- } \\
86\end{array}$ & 530 & $\begin{array}{r}10632 \\
5\end{array}$ & 1.5 & $\begin{array}{r}14.47 \\
66 \\
\end{array}$ & 1.7 & 1.2712 & 5.2 & $\begin{array}{r}0.133 \\
5\end{array}$ & $\begin{array}{r}4 . \\
9\end{array}$ & $\begin{array}{r}0.9 \\
5 \\
\end{array}$ & 807.6 & 37.0 & 832.9 & 29.3 & 901.0 & 34.0 & 807.6 & 37.0 & 89.6 \\
\hline $\begin{array}{l}\text { BU0753- } \\
100\end{array}$ & 224 & 44755 & 3.0 & $\begin{array}{r}14.63 \\
04\end{array}$ & 2.8 & 1.3073 & 3.3 & $\begin{array}{r}0.138 \\
7\end{array}$ & $\begin{array}{r}1 . \\
8\end{array}$ & $\begin{array}{r}0.5 \\
4\end{array}$ & 837.4 & 13.9 & 849.0 & 18.9 & 879.2 & 57.1 & 837.4 & 13.9 & 95.3 \\
\hline $\begin{array}{l}\text { BU0753- } \\
93\end{array}$ & $\begin{array}{r}100 \\
9\end{array}$ & $\begin{array}{r}17245 \\
0\end{array}$ & 5.6 & $\begin{array}{r}14.09 \\
97\end{array}$ & 1.4 & 1.4113 & 3.8 & $\begin{array}{r}0.144 \\
3\end{array}$ & $\begin{array}{r}3 . \\
6\end{array}$ & $\begin{array}{r}0.9 \\
4\end{array}$ & 869.0 & 29.3 & 893.7 & 22.9 & 955.2 & 27.8 & 869.0 & 29.3 & 91.0 \\
\hline $\begin{array}{l}\text { BU0753- } \\
64\end{array}$ & 830 & $\begin{array}{r}11861 \\
0\end{array}$ & 4.4 & $\begin{array}{r}13.55 \\
14\end{array}$ & 2.3 & 1.4725 & 4.2 & $\begin{array}{r}0.144 \\
7\end{array}$ & $\begin{array}{r}3 . \\
5\end{array}$ & $\begin{array}{r}0.8 \\
3\end{array}$ & 871.3 & 28.5 & 919.1 & 25.4 & $\begin{array}{r}1035 . \\
8\end{array}$ & 46.7 & 871.3 & 28.5 & 84.1 \\
\hline $\begin{array}{l}\text { BU0753- } \\
38\end{array}$ & 559 & $\begin{array}{r}16557 \\
0\end{array}$ & 0.7 & $\begin{array}{r}14.47 \\
54\end{array}$ & 1.2 & 1.3818 & 1.5 & $\begin{array}{r}0.145 \\
1\end{array}$ & $\begin{array}{r}1 . \\
0\end{array}$ & $\begin{array}{r}0.6 \\
5\end{array}$ & 873.2 & 8.2 & 881.2 & 9.0 & 901.2 & 23.9 & 873.2 & 8.2 & 96.9 \\
\hline $\begin{array}{l}\text { BU0753- } \\
16\end{array}$ & 424 & 97190 & 2.6 & $\begin{array}{r}14.61 \\
61\end{array}$ & 1.6 & 1.3707 & 1.9 & $\begin{array}{r}0.145 \\
3\end{array}$ & $\begin{array}{l}1 . \\
1\end{array}$ & $\begin{array}{r}0.5 \\
7 \\
\end{array}$ & 874.6 & 9.0 & 876.5 & 11.3 & 881.2 & 32.7 & 874.6 & 9.0 & 99.2 \\
\hline $\begin{array}{l}\text { BU0753- } \\
84\end{array}$ & 122 & $\begin{array}{r}38607 \\
5\end{array}$ & 1.7 & $\begin{array}{r}13.88 \\
98\end{array}$ & 4.4 & 1.4958 & 5.1 & $\begin{array}{r}0.150 \\
7\end{array}$ & $\begin{array}{r}2 . \\
6\end{array}$ & $\begin{array}{r}0.5 \\
1\end{array}$ & 904.8 & 22.1 & 928.7 & 31.0 & 985.8 & 89.0 & 904.8 & 22.1 & 91.8 \\
\hline $\begin{array}{l}\text { BU0753- } \\
69\end{array}$ & 288 & $\begin{array}{r}11998 \\
0\end{array}$ & 1.6 & $\begin{array}{r}13.83 \\
39\end{array}$ & 1.9 & 1.7014 & 2.9 & $\begin{array}{r}0.170 \\
7\end{array}$ & $\begin{array}{r}2 . \\
2\end{array}$ & $\begin{array}{r}0.7 \\
5\end{array}$ & $\begin{array}{r}1016 . \\
0\end{array}$ & 20.6 & 1009.1 & 18.7 & 994.0 & 39.4 & 994.0 & 39.4 & $\begin{array}{r}102 . \\
2\end{array}$ \\
\hline $\begin{array}{l}\text { BU0753- } \\
19\end{array}$ & 93 & 23410 & 2.2 & $\begin{array}{r}13.74 \\
80\end{array}$ & 4.2 & 1.6567 & 4.7 & $\begin{array}{r}0.165 \\
2\end{array}$ & $\begin{array}{r}2 . \\
0\end{array}$ & $\begin{array}{r}0.4 \\
4\end{array}$ & 985.6 & 18.6 & 992.1 & 29.5 & $\begin{array}{r}1006 . \\
6\end{array}$ & 84.9 & 1006.6 & 84.9 & 97.9 \\
\hline $\begin{array}{l}\text { BU0753- } \\
99\end{array}$ & 524 & 31040 & 8.6 & $\begin{array}{r}13.49 \\
06\end{array}$ & 4.7 & 1.5960 & 4.8 & $\begin{array}{r}0.156 \\
2\end{array}$ & $\begin{array}{l}1 . \\
0\end{array}$ & $\begin{array}{r}0.2 \\
1\end{array}$ & 935.4 & 8.7 & 968.7 & 29.8 & $\begin{array}{r}1044 . \\
9\end{array}$ & 94.1 & 1044.9 & 94.1 & 89.5 \\
\hline $\begin{array}{l}\text { BU0753- } \\
12\end{array}$ & 457 & $\begin{array}{r}19857 \\
5\end{array}$ & 6.5 & $\begin{array}{r}13.43 \\
23\end{array}$ & 2.4 & 1.7836 & 2.8 & $\begin{array}{r}0.173 \\
8\end{array}$ & $\begin{array}{l}1 . \\
4\end{array}$ & $\begin{array}{r}0.4 \\
9 \\
\end{array}$ & $\begin{array}{r}1032 . \\
8 \\
\end{array}$ & 13.2 & 1039.5 & 18.2 & $\begin{array}{r}1053 . \\
6 \\
\end{array}$ & 49.2 & 1053.6 & 49.2 & 98.0 \\
\hline BU0753-2 & 111 & 32625 & 1.8 & $\begin{array}{r}13.36 \\
44\end{array}$ & 3.0 & 1.7662 & 4.0 & $\begin{array}{r}0.171 \\
2\end{array}$ & $\begin{array}{r}2 . \\
7\end{array}$ & $\begin{array}{r}0.6 \\
8\end{array}$ & $\begin{array}{r}1018 . \\
7\end{array}$ & 25.7 & 1033.1 & 26.1 & $\begin{array}{r}1063 . \\
8\end{array}$ & 59.6 & 1063.8 & 59.6 & 95.8 \\
\hline $\begin{array}{l}\text { BU0753- } \\
70\end{array}$ & 324 & 93105 & 3.6 & $\begin{array}{r}13.33 \\
77\end{array}$ & 2.1 & 1.8097 & 2.4 & $\begin{array}{r}0.175 \\
1\end{array}$ & $\begin{array}{r}1 . \\
0\end{array}$ & $\begin{array}{r}0.4 \\
2\end{array}$ & $\begin{array}{r}1039 . \\
9\end{array}$ & 9.6 & 1049.0 & 15.4 & $\begin{array}{r}1067 . \\
8\end{array}$ & 42.8 & 1067.8 & 42.8 & 97.4 \\
\hline $\begin{array}{l}\text { BU0753- } \\
52\end{array}$ & 333 & $\begin{array}{r}13554 \\
5\end{array}$ & 6.1 & $\begin{array}{r}13.30 \\
18\end{array}$ & 1.4 & 1.7909 & 2.2 & $\begin{array}{r}0.172 \\
8\end{array}$ & $\begin{array}{l}1 . \\
7\end{array}$ & $\begin{array}{r}0.7 \\
6\end{array}$ & $\begin{array}{r}1027 . \\
4\end{array}$ & 15.9 & 1042.1 & 14.2 & $\begin{array}{r}1073 . \\
2\end{array}$ & 28.3 & 1073.2 & 28.3 & 95.7 \\
\hline $\begin{array}{l}\text { BU0753- } \\
10\end{array}$ & 433 & $\begin{array}{r}17233 \\
5\end{array}$ & 2.8 & $\begin{array}{r}13.28 \\
76\end{array}$ & 3.0 & 1.7304 & 4.8 & $\begin{array}{r}0.166 \\
8\end{array}$ & $\begin{array}{r}3 . \\
7\end{array}$ & $\begin{array}{r}0.7 \\
8\end{array}$ & 994.2 & 34.5 & 1019.9 & 31.0 & $\begin{array}{r}1075 . \\
4\end{array}$ & 61.1 & 1075.4 & 61.1 & 92.5 \\
\hline BU0753-4 & 168 & 46505 & 1.2 & $\begin{array}{r}13.28 \\
59\end{array}$ & 2.5 & 1.8175 & 3.4 & $\begin{array}{r}0.175 \\
1\end{array}$ & $\begin{array}{r}2 . \\
3\end{array}$ & $\begin{array}{r}0.6 \\
8\end{array}$ & $\begin{array}{r}1040 . \\
3\end{array}$ & 21.9 & 1051.8 & 22.0 & $\begin{array}{r}1075 . \\
6 \\
\end{array}$ & 49.6 & 1075.6 & 49.6 & 96.7 \\
\hline $\begin{array}{l}\text { BU0753- } \\
45\end{array}$ & 104 & 42835 & 1.6 & $\begin{array}{r}13.06 \\
76\end{array}$ & 2.6 & 2.0064 & 3.2 & $\begin{array}{r}0.190 \\
2\end{array}$ & $\begin{array}{r}1 . \\
9\end{array}$ & $\begin{array}{r}0.5 \\
9\end{array}$ & $\begin{array}{r}1122 . \\
2\end{array}$ & 19.3 & 1117.7 & 21.6 & $\begin{array}{r}1108 . \\
8\end{array}$ & 51.6 & 1108.8 & 51.6 & $\begin{array}{r}101 . \\
2\end{array}$ \\
\hline $\begin{array}{l}\text { BU0753- } \\
15\end{array}$ & 158 & 57850 & 1.3 & $\begin{array}{r}12.95 \\
89\end{array}$ & 2.2 & 1.8927 & 4.3 & $\begin{array}{r}0.177 \\
9\end{array}$ & $\begin{array}{r}3 . \\
8\end{array}$ & $\begin{array}{r}0.8 \\
6\end{array}$ & $\begin{array}{r}1055 . \\
4\end{array}$ & 36.5 & 1078.5 & 28.9 & $\begin{array}{r}1125 . \\
5\end{array}$ & 43.7 & 1125.5 & 43.7 & 93.8 \\
\hline $\begin{array}{l}\text { BU0753- } \\
33\end{array}$ & 200 & 58035 & 1.0 & $\begin{array}{r}12.92 \\
39\end{array}$ & 1.3 & 1.8783 & 3.2 & $\begin{array}{r}0.176 \\
1\end{array}$ & $\begin{array}{r}2 . \\
9\end{array}$ & $\begin{array}{r}0.9 \\
1\end{array}$ & $\begin{array}{r}1045 . \\
4\end{array}$ & 27.6 & 1073.5 & 20.9 & $\begin{array}{r}1130 . \\
9\end{array}$ & 26.3 & 1130.9 & 26.3 & 92.4 \\
\hline BU0753-3 & 125 & 46170 & 1.0 & $\begin{array}{r}12.84 \\
79\end{array}$ & 2.5 & 1.8901 & 3.3 & $\begin{array}{r}0.176 \\
1\end{array}$ & $\begin{array}{r}2 . \\
3\end{array}$ & $\begin{array}{r}0.6 \\
8\end{array}$ & $\begin{array}{r}1045 . \\
7\end{array}$ & 21.9 & 1077.6 & 22.2 & $\begin{array}{r}1142 . \\
6\end{array}$ & 48.7 & 1142.6 & 48.7 & 91.5 \\
\hline
\end{tabular}




\begin{tabular}{|c|c|c|c|c|c|c|c|c|c|c|c|c|c|c|c|c|c|c|c|}
\hline $\begin{array}{l}\text { BU0753- } \\
53\end{array}$ & 343 & $\begin{array}{r}17607 \\
0 \\
\end{array}$ & 1.1 & $\begin{array}{r}12.75 \\
93 \\
\end{array}$ & 1.6 & 2.0106 & 3.1 & $\begin{array}{r}0.186 \\
1 \\
\end{array}$ & $\begin{array}{r}2 . \\
6 \\
\end{array}$ & $\begin{array}{r}0.8 \\
5 \\
\end{array}$ & $\begin{array}{r}1100 . \\
0 \\
\end{array}$ & 26.5 & 1119.1 & 21.0 & $\begin{array}{r}1156 . \\
4 \\
\end{array}$ & 32.5 & 1156.4 & 32.5 & 95.1 \\
\hline $\begin{array}{l}\text { BU0753- } \\
18\end{array}$ & 506 & $\begin{array}{r}16248 \\
5\end{array}$ & 2.2 & $\begin{array}{r}12.70 \\
50\end{array}$ & 1.5 & 2.1283 & 2.0 & $\begin{array}{r}0.196 \\
1\end{array}$ & $\begin{array}{r}1 . \\
3\end{array}$ & $\begin{array}{r}0.6 \\
6\end{array}$ & $\begin{array}{r}1154 . \\
4\end{array}$ & 13.7 & 1158.0 & 13.5 & $\begin{array}{r}1164 . \\
8\end{array}$ & 28.9 & 1164.8 & 28.9 & 99.1 \\
\hline $\begin{array}{l}\text { BU0753- } \\
89\end{array}$ & 76 & 18305 & 1.3 & $\begin{array}{r}12.67 \\
95\end{array}$ & 2.1 & 2.0777 & 2.8 & $\begin{array}{r}0.191 \\
1\end{array}$ & $\begin{array}{r}1 . \\
8\end{array}$ & $\begin{array}{r}0.6 \\
5\end{array}$ & $\begin{array}{r}1127 . \\
1\end{array}$ & 18.5 & 1141.5 & 18.9 & $\begin{array}{r}1168 . \\
8\end{array}$ & 41.7 & 1168.8 & 41.7 & 96.4 \\
\hline $\begin{array}{l}\text { BU0753- } \\
80\end{array}$ & 138 & 46110 & 0.8 & $\begin{array}{r}12.66 \\
38 \\
\end{array}$ & 3.6 & 2.1228 & 3.7 & $\begin{array}{r}0.195 \\
0 \\
\end{array}$ & $\begin{array}{r}1 . \\
1\end{array}$ & $\begin{array}{r}0.2 \\
9 \\
\end{array}$ & $\begin{array}{r}1148 . \\
2\end{array}$ & 11.1 & 1156.2 & 25.6 & $\begin{array}{r}1171 . \\
2\end{array}$ & 70.5 & 1171.2 & 70.5 & 98.0 \\
\hline $\begin{array}{l}\text { BU0753- } \\
67\end{array}$ & 50 & 20505 & 1.1 & $\begin{array}{r}12.65 \\
41 \\
\end{array}$ & 4.8 & 2.0831 & 4.9 & $\begin{array}{r}0.191 \\
2 \\
\end{array}$ & $\begin{array}{r}1 . \\
0\end{array}$ & $\begin{array}{r}0.2 \\
0 \\
\end{array}$ & $\begin{array}{r}1127 . \\
7 \\
\end{array}$ & 10.3 & 1143.2 & 33.7 & $\begin{array}{r}1172 . \\
8 \\
\end{array}$ & 95.1 & 1172.8 & 95.1 & 96.2 \\
\hline $\begin{array}{l}\text { BU0753- } \\
41\end{array}$ & 122 & 9810 & 1.2 & $\begin{array}{r}12.62 \\
84 \\
\end{array}$ & 3.1 & 2.0054 & 5.0 & $\begin{array}{r}0.183 \\
7 \\
\end{array}$ & $\begin{array}{r}3 . \\
9\end{array}$ & $\begin{array}{r}0.7 \\
8 \\
\end{array}$ & $\begin{array}{r}1087 . \\
0\end{array}$ & 39.3 & 1117.3 & 34.1 & $\begin{array}{r}1176 . \\
8\end{array}$ & 62.3 & 1176.8 & 62.3 & 92.4 \\
\hline $\begin{array}{l}\text { BU0753- } \\
28\end{array}$ & 169 & 43125 & 1.0 & $\begin{array}{r}12.58 \\
57\end{array}$ & 4.8 & 2.0081 & 5.3 & $\begin{array}{r}0.183 \\
3\end{array}$ & $\begin{array}{r}2 . \\
2\end{array}$ & $\begin{array}{r}0.4 \\
1\end{array}$ & $\begin{array}{r}1085 . \\
0\end{array}$ & 21.9 & 1118.3 & 35.8 & $\begin{array}{r}1183 . \\
5\end{array}$ & 94.9 & 1183.5 & 94.9 & 91.7 \\
\hline $\begin{array}{l}\text { BU0753- } \\
22\end{array}$ & 243 & 71040 & 1.8 & $\begin{array}{r}12.48 \\
13 \\
\end{array}$ & 3.4 & 2.2216 & 3.7 & $\begin{array}{r}0.201 \\
1 \\
\end{array}$ & $\begin{array}{r}1 . \\
5\end{array}$ & $\begin{array}{r}0.4 \\
0 \\
\end{array}$ & $\begin{array}{r}1181 . \\
3 \\
\end{array}$ & 15.9 & 1187.9 & 25.9 & $\begin{array}{r}1199 . \\
9 \\
\end{array}$ & 67.1 & 1199.9 & 67.1 & 98.4 \\
\hline $\begin{array}{l}\text { BU0753- } \\
13\end{array}$ & 262 & $\begin{array}{r}11401 \\
5 \\
\end{array}$ & 3.6 & $\begin{array}{r}12.23 \\
19 \\
\end{array}$ & 3.8 & 2.3401 & 3.9 & $\begin{array}{r}0.207 \\
6 \\
\end{array}$ & $\begin{array}{r}1 . \\
0\end{array}$ & $\begin{array}{r}0.2 \\
6\end{array}$ & $\begin{array}{r}1216 . \\
0\end{array}$ & 11.1 & 1224.6 & 27.8 & $\begin{array}{r}1239 . \\
6\end{array}$ & 73.9 & 1239.6 & 73.9 & 98.1 \\
\hline $\begin{array}{l}\text { BU0753- } \\
66\end{array}$ & 390 & 80745 & 1.5 & $\begin{array}{r}11.97 \\
84 \\
\end{array}$ & 1.1 & 2.3260 & 3.2 & $\begin{array}{r}0.202 \\
1 \\
\end{array}$ & $\begin{array}{r}3 . \\
0\end{array}$ & $\begin{array}{r}0.9 \\
4 \\
\end{array}$ & $\begin{array}{r}1186 . \\
5 \\
\end{array}$ & 32.9 & 1220.3 & 22.9 & $\begin{array}{r}1280 . \\
5 \\
\end{array}$ & 21.2 & 1280.5 & 21.2 & 92.7 \\
\hline $\begin{array}{l}\text { BU0753- } \\
72\end{array}$ & 113 & 68300 & 2.1 & $\begin{array}{r}11.86 \\
22\end{array}$ & 4.4 & 2.5015 & 4.6 & $\begin{array}{r}0.215 \\
2\end{array}$ & $\begin{array}{r}1 . \\
4\end{array}$ & $\begin{array}{r}0.3 \\
1\end{array}$ & $\begin{array}{r}1256 . \\
5\end{array}$ & 16.0 & 1272.5 & 33.3 & $\begin{array}{r}1299 . \\
5\end{array}$ & 85.0 & 1299.5 & 85.0 & 96.7 \\
\hline $\begin{array}{l}\text { BU0753- } \\
46\end{array}$ & 301 & $\begin{array}{r}15776 \\
5\end{array}$ & 2.5 & $\begin{array}{r}11.38 \\
50\end{array}$ & 3.0 & 2.6322 & 3.8 & $\begin{array}{r}0.217 \\
3\end{array}$ & $\begin{array}{r}2 . \\
3\end{array}$ & $\begin{array}{r}0.6 \\
1\end{array}$ & $\begin{array}{r}1267 . \\
9\end{array}$ & 26.6 & 1309.7 & 27.8 & $\begin{array}{r}1378 . \\
9\end{array}$ & 57.5 & 1378.9 & 57.5 & 91.9 \\
\hline $\begin{array}{l}\text { BU0753- } \\
39\end{array}$ & 346 & $\begin{array}{r}10610 \\
0 \\
\end{array}$ & 1.9 & $\begin{array}{r}11.05 \\
92 \\
\end{array}$ & 1.9 & 3.0407 & 2.6 & $\begin{array}{r}0.243 \\
9 \\
\end{array}$ & $\begin{array}{r}1 . \\
8\end{array}$ & $\begin{array}{r}0.6 \\
9 \\
\end{array}$ & $\begin{array}{r}1406 . \\
9\end{array}$ & 22.2 & 1417.9 & 19.6 & $\begin{array}{r}1434 . \\
5\end{array}$ & 35.7 & 1434.5 & 35.7 & 98.1 \\
\hline $\begin{array}{l}\text { BU0753- } \\
35\end{array}$ & 424 & $\begin{array}{r}14384 \\
0 \\
\end{array}$ & 2.6 & $\begin{array}{r}11.05 \\
48 \\
\end{array}$ & 1.2 & 3.0008 & 2.5 & $\begin{array}{r}0.240 \\
6 \\
\end{array}$ & $\begin{array}{r}2 . \\
2 \\
\end{array}$ & $\begin{array}{r}0.8 \\
8 \\
\end{array}$ & $\begin{array}{r}1389 . \\
8 \\
\end{array}$ & 27.0 & 1407.8 & 18.8 & $\begin{array}{r}1435 . \\
2 \\
\end{array}$ & 22.7 & 1435.2 & 22.7 & 96.8 \\
\hline $\begin{array}{l}\text { BU0753- } \\
78\end{array}$ & 294 & $\begin{array}{r}12934 \\
0 \\
\end{array}$ & 1.9 & $\begin{array}{r}10.93 \\
06 \\
\end{array}$ & 4.5 & 2.9084 & 4.6 & $\begin{array}{r}0.230 \\
6 \\
\end{array}$ & $\begin{array}{r}1 . \\
0\end{array}$ & $\begin{array}{r}0.2 \\
2 \\
\end{array}$ & $\begin{array}{r}1337 . \\
5 \\
\end{array}$ & 12.1 & 1384.1 & 34.6 & $\begin{array}{r}1456 . \\
7 \\
\end{array}$ & 84.9 & 1456.7 & 84.9 & 91.8 \\
\hline $\begin{array}{l}\text { BU0753- } \\
36\end{array}$ & 983 & $\begin{array}{r}21197 \\
5\end{array}$ & 8.8 & $\begin{array}{r}10.44 \\
75\end{array}$ & 1.5 & 2.6735 & 4.4 & $\begin{array}{r}0.202 \\
6\end{array}$ & $\begin{array}{r}4 . \\
1\end{array}$ & $\begin{array}{r}0.9 \\
4\end{array}$ & $\begin{array}{r}1189 . \\
1\end{array}$ & 44.6 & 1321.2 & 32.4 & $\begin{array}{r}1542 . \\
2\end{array}$ & 28.6 & 1542.2 & 28.6 & 77.1 \\
\hline $\begin{array}{l}\text { BU0753- } \\
58\end{array}$ & 177 & 66040 & 2.0 & $\begin{array}{r}10.37 \\
89 \\
\end{array}$ & 1.3 & 3.6273 & 1.8 & $\begin{array}{r}0.273 \\
0 \\
\end{array}$ & $\begin{array}{r}1 . \\
3\end{array}$ & $\begin{array}{r}0.7 \\
1 \\
\end{array}$ & $\begin{array}{r}1556 . \\
2 \\
\end{array}$ & 17.6 & 1555.5 & 14.3 & $\begin{array}{r}1554 . \\
6 \\
\end{array}$ & 23.8 & 1554.6 & 23.8 & $\begin{array}{r}100 . \\
1\end{array}$ \\
\hline $\begin{array}{l}\text { BU0753- } \\
74\end{array}$ & 739 & $\begin{array}{r}15752 \\
5 \\
\end{array}$ & 1.7 & $\begin{array}{r}10.19 \\
40 \\
\end{array}$ & 2.2 & 3.6581 & 2.5 & $\begin{array}{r}0.270 \\
5 \\
\end{array}$ & $\begin{array}{r}1 . \\
3\end{array}$ & $\begin{array}{r}0.4 \\
9 \\
\end{array}$ & $\begin{array}{r}1543 . \\
1 \\
\end{array}$ & 17.2 & 1562.3 & 20.2 & $\begin{array}{r}1588 . \\
2 \\
\end{array}$ & 41.3 & 1588.2 & 41.3 & 97.2 \\
\hline $\begin{array}{l}\text { BU0753- } \\
85\end{array}$ & 825 & $\begin{array}{r}2518 \\
0 \\
\end{array}$ & 2.0 & $\begin{array}{r}10.07 \\
85 \\
\end{array}$ & 2.9 & 3.9271 & 3.0 & $\begin{array}{r}0.287 \\
1 \\
\end{array}$ & $\begin{array}{r}1 . \\
0\end{array}$ & $\begin{array}{r}0.3 \\
3 \\
\end{array}$ & $\begin{array}{r}1626 . \\
8 \\
\end{array}$ & 14.4 & 1619.3 & 24.5 & $\begin{array}{r}1609 . \\
5 \\
\end{array}$ & 53.3 & 1609.5 & 53.3 & $\begin{array}{r}101 . \\
1\end{array}$ \\
\hline $\begin{array}{l}\text { BU0753- } \\
30\end{array}$ & 162 & 81005 & 2.1 & $\begin{array}{r}10.06 \\
97\end{array}$ & 1.9 & 3.5787 & 2.8 & $\begin{array}{r}0.261 \\
4\end{array}$ & $\begin{array}{r}2 . \\
1\end{array}$ & $\begin{array}{r}0.7 \\
4\end{array}$ & $\begin{array}{r}1496 . \\
8\end{array}$ & 28.1 & 1544.8 & 22.5 & $\begin{array}{r}1611 . \\
1\end{array}$ & 35.6 & 1611.1 & 35.6 & 92.9 \\
\hline $\begin{array}{l}\text { BU0753- } \\
42\end{array}$ & 317 & $\begin{array}{r}18418 \\
5 \\
\end{array}$ & 1.8 & $\begin{array}{r}9.990 \\
6 \\
\end{array}$ & 2.3 & 3.5775 & 3.4 & $\begin{array}{r}0.259 \\
2 \\
\end{array}$ & $\begin{array}{r}2 . \\
4 \\
\end{array}$ & $\begin{array}{r}0.7 \\
3\end{array}$ & $\begin{array}{r}1485 . \\
9\end{array}$ & 32.4 & 1544.6 & 26.6 & $\begin{array}{r}1625 . \\
8\end{array}$ & 42.8 & 1625.8 & 42.8 & 91.4 \\
\hline $\begin{array}{l}\text { BU0753- } \\
48\end{array}$ & 338 & $\begin{array}{r}17853 \\
5\end{array}$ & 2.2 & $\begin{array}{r}9.823 \\
2\end{array}$ & 2.6 & 3.9083 & 2.8 & $\begin{array}{r}0.278 \\
4\end{array}$ & $\begin{array}{r}1 . \\
1\end{array}$ & $\begin{array}{r}0.3 \\
9\end{array}$ & $\begin{array}{r}1583 . \\
5\end{array}$ & 15.3 & 1615.4 & 22.8 & $\begin{array}{r}1657 . \\
2\end{array}$ & 48.2 & 1657.2 & 48.2 & 95.6 \\
\hline $\begin{array}{l}\text { BU0753- } \\
14\end{array}$ & 430 & $\begin{array}{r}32495 \\
5 \\
\end{array}$ & 5.1 & $\begin{array}{r}9.704 \\
7 \\
\end{array}$ & 4.8 & 4.2363 & 4.9 & $\begin{array}{r}0.298 \\
2 \\
\end{array}$ & $\begin{array}{r}1 . \\
2\end{array}$ & $\begin{array}{r}0.2 \\
4 \\
\end{array}$ & $\begin{array}{r}1682 . \\
2 \\
\end{array}$ & 17.5 & 1681.1 & 40.5 & $\begin{array}{r}1679 . \\
6 \\
\end{array}$ & 88.5 & 1679.6 & 88.5 & $\begin{array}{r}100 . \\
2\end{array}$ \\
\hline $\begin{array}{l}\text { BU0753- } \\
96\end{array}$ & 480 & 82185 & 1.4 & $\begin{array}{r}9.510 \\
5 \\
\end{array}$ & 2.3 & 3.8852 & 2.6 & $\begin{array}{r}0.268 \\
0 \\
\end{array}$ & $\begin{array}{r}1 . \\
2\end{array}$ & $\begin{array}{r}0.4 \\
5 \\
\end{array}$ & $\begin{array}{r}1530 . \\
6 \\
\end{array}$ & 15.9 & 1610.6 & 21.1 & $\begin{array}{r}1716 . \\
9\end{array}$ & 42.8 & 1716.9 & 42.8 & 89.2 \\
\hline BU0753- & 264 & 11824 & 2.5 & 9.339 & 1.0 & 4.3561 & 1.7 & 0.295 & 1. & 0.8 & 1666. & 20.7 & 1704.1 & 14.3 & 1750. & 18.3 & 1750.2 & 18.3 & 95.2 \\
\hline
\end{tabular}




\begin{tabular}{|c|c|c|c|c|c|c|c|c|c|c|c|c|c|c|c|c|c|c|c|}
\hline 29 & & 5 & & 4 & & & & 1 & 4 & 2 & 8 & & & & 2 & & & & \\
\hline $\begin{array}{l}\text { BU0753- } \\
40\end{array}$ & 295 & $\begin{array}{r}15483 \\
5 \\
\end{array}$ & 1.1 & $\begin{array}{r}6.400 \\
2 \\
\end{array}$ & 2.8 & 8.9788 & 3.4 & $\begin{array}{r}0.416 \\
8 \\
\end{array}$ & $\begin{array}{r}1 . \\
9\end{array}$ & $\begin{array}{r}0.5 \\
6\end{array}$ & $\begin{array}{r}2245 . \\
9\end{array}$ & 36.0 & 2335.9 & 30.8 & $\begin{array}{r}2415 . \\
4\end{array}$ & 47.4 & 2415.4 & 47.4 & 93.0 \\
\hline $\begin{array}{l}\text { BU0753- } \\
56\end{array}$ & 947 & $\begin{array}{r}38766 \\
5 \\
\end{array}$ & 2.9 & $\begin{array}{r}6.339 \\
2 \\
\end{array}$ & 2.0 & 8.1926 & 5.3 & $\begin{array}{r}0.376 \\
7 \\
\end{array}$ & $\begin{array}{r}5 . \\
0 \\
\end{array}$ & $\begin{array}{r}0.9 \\
3 \\
\end{array}$ & $\begin{array}{r}2060 . \\
7 \\
\end{array}$ & 87.3 & 2252.5 & 48.4 & $\begin{array}{r}2431 . \\
6 \\
\end{array}$ & 34.1 & 2431.6 & 34.1 & 84.7 \\
\hline $\begin{array}{l}\text { BU0753- } \\
61\end{array}$ & 386 & $\begin{array}{r}23369 \\
5 \\
\end{array}$ & 2.2 & $\begin{array}{r}6.300 \\
8 \\
\end{array}$ & 1.0 & 9.0582 & 2.3 & $\begin{array}{r}0.413 \\
9 \\
\end{array}$ & $\begin{array}{r}2 . \\
1\end{array}$ & $\begin{array}{r}0.9 \\
0 \\
\end{array}$ & $\begin{array}{r}2232 . \\
9 \\
\end{array}$ & 39.8 & 2343.9 & 21.4 & $\begin{array}{r}2441 . \\
9\end{array}$ & 16.9 & 2441.9 & 16.9 & 91.4 \\
\hline $\begin{array}{l}\text { BU0753- } \\
34\end{array}$ & 314 & $\begin{array}{r}20699 \\
5 \\
\end{array}$ & 2.2 & $\begin{array}{r}6.203 \\
5 \\
\end{array}$ & 1.3 & 9.1910 & 2.8 & $\begin{array}{r}0.413 \\
5 \\
\end{array}$ & $\begin{array}{r}2 . \\
5\end{array}$ & $\begin{array}{r}0.8 \\
9 \\
\end{array}$ & $\begin{array}{r}2231 . \\
0 \\
\end{array}$ & 47.5 & 2357.2 & 25.9 & $\begin{array}{r}2468 . \\
3 \\
\end{array}$ & 21.8 & 2468.3 & 21.8 & 90.4 \\
\hline $\begin{array}{l}\text { BU0753- } \\
23\end{array}$ & 551 & $\begin{array}{r}27123 \\
0 \\
\end{array}$ & 1.9 & $\begin{array}{r}6.158 \\
1 \\
\end{array}$ & 1.3 & 9.7681 & 2.3 & $\begin{array}{r}0.436 \\
3 \\
\end{array}$ & $\begin{array}{r}1 . \\
9\end{array}$ & $\begin{array}{r}0.8 \\
4 \\
\end{array}$ & $\begin{array}{r}2333 . \\
9 \\
\end{array}$ & 37.2 & 2413.1 & 21.0 & $\begin{array}{r}2480 . \\
7 \\
\end{array}$ & 21.1 & 2480.7 & 21.1 & 94.1 \\
\hline $\begin{array}{l}\text { BU0753- } \\
87\end{array}$ & 677 & $\begin{array}{r}1544 \\
0 \\
\end{array}$ & 1.2 & $\begin{array}{r}5.967 \\
5 \\
\end{array}$ & 1.1 & 9.7660 & 3.6 & $\begin{array}{r}0.422 \\
7 \\
\end{array}$ & $\begin{array}{r}3 . \\
4 \\
\end{array}$ & $\begin{array}{r}0.9 \\
5 \\
\end{array}$ & $\begin{array}{r}2272 . \\
6 \\
\end{array}$ & 65.9 & 2413.0 & 33.3 & $\begin{array}{r}2533 . \\
6 \\
\end{array}$ & 18.5 & 2533.6 & 18.5 & 89.7 \\
\hline $\begin{array}{l}\text { BU0753- } \\
11\end{array}$ & 112 & $\begin{array}{r}10168 \\
0 \\
\end{array}$ & 1.5 & $\begin{array}{r}5.186 \\
5 \\
\end{array}$ & 2.1 & $\begin{array}{r}12.198 \\
0 \\
\end{array}$ & 3.5 & $\begin{array}{r}0.458 \\
8 \\
\end{array}$ & $\begin{array}{r}2 . \\
8\end{array}$ & $\begin{array}{r}0.8 \\
0 \\
\end{array}$ & $\begin{array}{r}2434 . \\
4 \\
\end{array}$ & 56.8 & 2619.8 & 32.8 & $\begin{array}{r}2766 . \\
3 \\
\end{array}$ & 34.3 & 2766.3 & 34.3 & 88.0 \\
\hline $\begin{array}{l}\text { BU0753- } \\
43\end{array}$ & 316 & $\begin{array}{r}25934 \\
5 \\
\end{array}$ & 1.4 & $\begin{array}{r}5.180 \\
2 \\
\end{array}$ & 1.2 & $\begin{array}{r}12.915 \\
2 \\
\end{array}$ & 1.5 & $\begin{array}{r}0.485 \\
2 \\
\end{array}$ & $\begin{array}{c}1 . \\
0\end{array}$ & $\begin{array}{r}0.6 \\
5 \\
\end{array}$ & $\begin{array}{r}2550 . \\
0 \\
\end{array}$ & 21.1 & 2673.5 & 14.6 & $\begin{array}{r}2768 . \\
3 \\
\end{array}$ & 19.4 & 2768.3 & 19.4 & 92.1 \\
\hline $\begin{array}{l}\text { BU0753- } \\
57\end{array}$ & 170 & $\begin{array}{r}12310 \\
0 \\
\end{array}$ & 2.1 & $\begin{array}{r}5.083 \\
4 \\
\end{array}$ & 1.4 & $\begin{array}{r}13.984 \\
0 \\
\end{array}$ & 2.9 & $\begin{array}{r}0.515 \\
6 \\
\end{array}$ & $\begin{array}{r}2 . \\
5\end{array}$ & $\begin{array}{r}0.8 \\
7 \\
\end{array}$ & $\begin{array}{r}2680 . \\
3 \\
\end{array}$ & 54.2 & 2748.6 & 27.1 & $\begin{array}{r}2799 . \\
2 \\
\end{array}$ & 23.4 & 2799.2 & 23.4 & 95.8 \\
\hline $\begin{array}{l}\text { BU0753- } \\
65\end{array}$ & 178 & $\begin{array}{r}12591 \\
5 \\
\end{array}$ & 1.3 & $\begin{array}{r}5.029 \\
1 \\
\end{array}$ & 1.5 & $\begin{array}{r}14.111 \\
5 \\
\end{array}$ & 1.9 & $\begin{array}{r}0.514 \\
7 \\
\end{array}$ & $\begin{array}{r}1 . \\
2\end{array}$ & $\begin{array}{r}0.6 \\
1 \\
\end{array}$ & $\begin{array}{r}2676 . \\
7\end{array}$ & 25.6 & 2757.2 & 18.3 & $\begin{array}{r}2816 . \\
7\end{array}$ & 25.0 & 2816.7 & 25.0 & 95.0 \\
\hline $\begin{array}{l}\text { BU0753- } \\
91\end{array}$ & 145 & 82980 & 1.8 & $\begin{array}{r}5.026 \\
9 \\
\end{array}$ & 1.0 & $\begin{array}{r}13.981 \\
4 \\
\end{array}$ & 2.1 & $\begin{array}{r}0.509 \\
7 \\
\end{array}$ & $\begin{array}{c}1 . \\
9 \\
\end{array}$ & $\begin{array}{r}0.8 \\
8 \\
\end{array}$ & $\begin{array}{r}2655 . \\
5 \\
\end{array}$ & 41.1 & 2748.4 & 20.3 & $\begin{array}{r}2817 . \\
4 \\
\end{array}$ & 16.3 & 2817.4 & 16.3 & 94.3 \\
\hline $\begin{array}{l}\text { BU0753- } \\
50\end{array}$ & 237 & $\begin{array}{r}12222 \\
0 \\
\end{array}$ & 2.0 & $\begin{array}{r}5.015 \\
6 \\
\end{array}$ & 1.1 & $\begin{array}{r}13.712 \\
0 \\
\end{array}$ & 1.7 & $\begin{array}{r}0.498 \\
8 \\
\end{array}$ & $\begin{array}{r}1 . \\
3\end{array}$ & $\begin{array}{r}0.7 \\
4 \\
\end{array}$ & $\begin{array}{r}2608 . \\
6 \\
\end{array}$ & 26.8 & 2730.0 & 15.9 & $\begin{array}{r}2821 . \\
1 \\
\end{array}$ & 18.3 & 2821.1 & 18.3 & 92.5 \\
\hline BU0753-8 & 159 & $\begin{array}{r}13598 \\
5 \\
\end{array}$ & 1.8 & $\begin{array}{r}4.986 \\
4 \\
\end{array}$ & 2.9 & $\begin{array}{r}15.375 \\
5 \\
\end{array}$ & 3.1 & $\begin{array}{r}0.556 \\
1 \\
\end{array}$ & $\begin{array}{r}1 . \\
0 \\
\end{array}$ & $\begin{array}{r}0.3 \\
2 \\
\end{array}$ & $\begin{array}{r}2850 . \\
3 \\
\end{array}$ & 23.0 & 2838.8 & 29.3 & $\begin{array}{r}2830 . \\
6 \\
\end{array}$ & 47.5 & 2830.6 & 47.5 & $\begin{array}{r}100 . \\
7\end{array}$ \\
\hline $\begin{array}{l}\text { BU0753- } \\
55\end{array}$ & 131 & $\begin{array}{r}15389 \\
5 \\
\end{array}$ & 1.7 & $\begin{array}{r}4.807 \\
1 \\
\end{array}$ & 2.6 & $\begin{array}{r}16.482 \\
2 \\
\end{array}$ & 3.4 & $\begin{array}{r}0.574 \\
6 \\
\end{array}$ & $\begin{array}{r}2 . \\
3\end{array}$ & $\begin{array}{r}0.6 \\
7 \\
\end{array}$ & $\begin{array}{r}2926 . \\
8 \\
\end{array}$ & 54.1 & 2905.2 & 33.0 & $\begin{array}{r}2890 . \\
2\end{array}$ & 41.6 & 2890.2 & 41.6 & $\begin{array}{r}101 . \\
3\end{array}$ \\
\hline $\begin{array}{l}\text { BU0754- } \\
73\end{array}$ & $\begin{array}{r}106 \\
4\end{array}$ & 82955 & 0.9 & $\begin{array}{r}17.20 \\
05\end{array}$ & 2.6 & 0.4397 & 3.5 & $\begin{array}{r}0.054 \\
8\end{array}$ & $\begin{array}{r}2 . \\
4\end{array}$ & $\begin{array}{r}0.6 \\
8\end{array}$ & 344.2 & 7.9 & 370.0 & 10.8 & 534.9 & 56.1 & 344.2 & 7.9 & NA \\
\hline $\begin{array}{l}\text { BU0754- } \\
98\end{array}$ & 381 & 8015 & 1.0 & $\begin{array}{r}13.86 \\
55\end{array}$ & $\begin{array}{r}17 . \\
0\end{array}$ & 0.5702 & 17.4 & $\begin{array}{r}0.057 \\
3\end{array}$ & $\begin{array}{r}3 . \\
3\end{array}$ & $\begin{array}{r}0.1 \\
9\end{array}$ & 359.4 & 11.5 & 458.2 & 64.1 & 989.3 & $\begin{array}{r}349 . \\
1\end{array}$ & 359.4 & 11.5 & NA \\
\hline $\begin{array}{l}\text { BU0754- } \\
58\end{array}$ & $\begin{array}{r}189 \\
5 \\
\end{array}$ & $\begin{array}{r}21875 \\
5 \\
\end{array}$ & 3.1 & $\begin{array}{r}17.49 \\
87 \\
\end{array}$ & 2.9 & 0.4850 & 3.1 & $\begin{array}{r}0.061 \\
6 \\
\end{array}$ & $\begin{array}{r}1 . \\
3\end{array}$ & $\begin{array}{r}0.4 \\
0 \\
\end{array}$ & 385.1 & 4.7 & 401.5 & 10.4 & 497.2 & 63.5 & 385.1 & 4.7 & NA \\
\hline $\begin{array}{l}\text { BU0754- } \\
56\end{array}$ & 946 & 24565 & 2.2 & $\begin{array}{r}15.72 \\
64 \\
\end{array}$ & 1.7 & 0.5533 & 3.3 & $\begin{array}{r}0.063 \\
1 \\
\end{array}$ & $\begin{array}{r}2 . \\
8 \\
\end{array}$ & $\begin{array}{r}0.8 \\
6 \\
\end{array}$ & 394.5 & 10.8 & 447.2 & 11.8 & 727.9 & 35.7 & 394.5 & 10.8 & NA \\
\hline $\begin{array}{l}\text { BU0754- } \\
44\end{array}$ & 827 & 84140 & 1.0 & $\begin{array}{r}17.65 \\
95 \\
\end{array}$ & 1.3 & 0.5009 & 4.2 & $\begin{array}{r}0.064 \\
2 \\
\end{array}$ & $\begin{array}{r}4 . \\
0\end{array}$ & $\begin{array}{r}0.9 \\
5 \\
\end{array}$ & 400.9 & 15.4 & 412.3 & 14.1 & 477.0 & 29.4 & 400.9 & 15.4 & NA \\
\hline BU0754-3 & $\begin{array}{r}123 \\
3\end{array}$ & 90935 & 2.1 & $\begin{array}{r}17.41 \\
11\end{array}$ & 2.3 & 0.5100 & 2.7 & $\begin{array}{r}0.064 \\
4\end{array}$ & $\begin{array}{r}1 . \\
4\end{array}$ & $\begin{array}{r}0.5 \\
2\end{array}$ & 402.3 & 5.5 & 418.5 & 9.2 & 508.2 & 50.4 & 402.3 & 5.5 & NA \\
\hline $\begin{array}{l}\text { BU0754- } \\
37\end{array}$ & 629 & 67090 & 1.8 & $\begin{array}{r}17.37 \\
75 \\
\end{array}$ & 1.3 & 0.5161 & 2.4 & $\begin{array}{r}0.065 \\
0 \\
\end{array}$ & $\begin{array}{r}2 . \\
0\end{array}$ & $\begin{array}{r}0.8 \\
3 \\
\end{array}$ & 406.3 & 7.8 & 422.6 & 8.3 & 512.5 & 29.5 & 406.3 & 7.8 & NA \\
\hline $\begin{array}{l}\text { BU0754- } \\
26\end{array}$ & $\begin{array}{r}111 \\
3\end{array}$ & 39035 & 2.4 & $\begin{array}{r}16.58 \\
40\end{array}$ & 4.5 & 0.5443 & 6.2 & $\begin{array}{r}0.065 \\
5\end{array}$ & $\begin{array}{r}4 . \\
3\end{array}$ & $\begin{array}{r}0.6 \\
9\end{array}$ & 408.8 & 16.9 & 441.2 & 22.2 & 614.3 & 97.2 & 408.8 & 16.9 & NA \\
\hline $\begin{array}{l}\text { BU0754- } \\
62\end{array}$ & 618 & $\begin{array}{r}10427 \\
0 \\
\end{array}$ & 2.6 & $\begin{array}{r}17.61 \\
10 \\
\end{array}$ & 3.1 & 0.5342 & 5.4 & $\begin{array}{r}0.068 \\
2 \\
\end{array}$ & $\begin{array}{r}4 . \\
5\end{array}$ & $\begin{array}{r}0.8 \\
2 \\
\end{array}$ & 425.5 & 18.4 & 434.6 & 19.3 & 483.1 & 68.7 & 425.5 & 18.4 & NA \\
\hline
\end{tabular}




\begin{tabular}{|c|c|c|c|c|c|c|c|c|c|c|c|c|c|c|c|c|c|c|c|}
\hline $\begin{array}{l}\text { BU0754- } \\
54\end{array}$ & $\begin{array}{r}275 \\
0 \\
\end{array}$ & $\begin{array}{r}14476 \\
0 \\
\end{array}$ & 2.7 & $\begin{array}{r}17.37 \\
43 \\
\end{array}$ & 2.7 & 0.5448 & 6.9 & $\begin{array}{r}0.068 \\
7 \\
\end{array}$ & $\begin{array}{r}6 . \\
4 \\
\end{array}$ & $\begin{array}{r}0.9 \\
2 \\
\end{array}$ & 428.0 & 26.4 & 441.6 & 24.8 & 512.9 & 59.8 & 428.0 & 26.4 & NA \\
\hline $\begin{array}{l}\text { BU0754- } \\
84\end{array}$ & $\begin{array}{r}102 \\
6 \\
\end{array}$ & $\begin{array}{r}18312 \\
5 \\
\end{array}$ & 12.1 & $\begin{array}{r}16.36 \\
89 \\
\end{array}$ & 3.1 & 0.5977 & 3.3 & $\begin{array}{r}0.071 \\
0 \\
\end{array}$ & $\begin{array}{r}1 . \\
3\end{array}$ & $\begin{array}{r}0.3 \\
7 \\
\end{array}$ & 441.9 & 5.3 & 475.8 & 12.7 & 642.4 & 66.4 & 441.9 & 5.3 & NA \\
\hline $\begin{array}{l}\text { BU0754- } \\
69\end{array}$ & $\begin{array}{r}379 \\
6 \\
\end{array}$ & $\begin{array}{r}31408 \\
5 \\
\end{array}$ & 14.5 & $\begin{array}{r}17.67 \\
75 \\
\end{array}$ & 1.4 & 0.5547 & 2.3 & $\begin{array}{r}0.071 \\
1 \\
\end{array}$ & $\begin{array}{r}1 . \\
9\end{array}$ & $\begin{array}{r}0.8 \\
0 \\
\end{array}$ & 442.9 & 7.9 & 448.1 & 8.4 & 474.7 & 31.0 & 442.9 & 7.9 & NA \\
\hline $\begin{array}{l}\text { BU0754- } \\
82\end{array}$ & $\begin{array}{r}216 \\
1 \\
\end{array}$ & $\begin{array}{r}27481 \\
0 \\
\end{array}$ & 3.2 & $\begin{array}{r}17.75 \\
27 \\
\end{array}$ & 3.4 & 0.5548 & 4.1 & $\begin{array}{r}0.071 \\
4 \\
\end{array}$ & $\begin{array}{r}2 . \\
4 \\
\end{array}$ & $\begin{array}{r}0.5 \\
7 \\
\end{array}$ & 444.8 & 10.1 & 448.1 & 15.0 & 465.4 & 75.6 & 444.8 & 10.1 & NA \\
\hline BU0754-7 & $\begin{array}{r}663 \\
4 \\
\end{array}$ & $\begin{array}{r}44762 \\
5 \\
\end{array}$ & 47.9 & $\begin{array}{r}17.37 \\
77 \\
\end{array}$ & 2.2 & 0.5673 & 3.1 & $\begin{array}{r}0.071 \\
5 \\
\end{array}$ & $\begin{array}{r}2 . \\
1 \\
\end{array}$ & $\begin{array}{r}0.6 \\
9 \\
\end{array}$ & 445.2 & 9.2 & 456.3 & 11.4 & 512.5 & 49.2 & 445.2 & 9.2 & NA \\
\hline $\begin{array}{l}\text { BU0754- } \\
65\end{array}$ & 853 & $\begin{array}{r}11300 \\
0 \\
\end{array}$ & 1.3 & $\begin{array}{r}17.23 \\
99 \\
\end{array}$ & 2.6 & 0.5735 & 2.7 & $\begin{array}{r}0.071 \\
7 \\
\end{array}$ & $\begin{array}{r}1 . \\
0\end{array}$ & $\begin{array}{r}0.3 \\
6 \\
\end{array}$ & 446.4 & 4.3 & 460.3 & 10.2 & 529.9 & 56.1 & 446.4 & 4.3 & NA \\
\hline $\begin{array}{l}\text { BU0754- } \\
79\end{array}$ & $\begin{array}{r}105 \\
9 \\
\end{array}$ & $\begin{array}{r}19132 \\
0 \\
\end{array}$ & 13.5 & $\begin{array}{r}17.20 \\
75 \\
\end{array}$ & 3.6 & 0.5783 & 5.4 & $\begin{array}{r}0.072 \\
2 \\
\end{array}$ & $\begin{array}{r}4 . \\
0\end{array}$ & $\begin{array}{r}0.7 \\
5 \\
\end{array}$ & 449.3 & 17.5 & 463.4 & 20.0 & 534.0 & 77.7 & 449.3 & 17.5 & NA \\
\hline $\begin{array}{l}\text { BU0754- } \\
50\end{array}$ & $\begin{array}{r}703 \\
6 \\
\end{array}$ & $\begin{array}{r}52708 \\
0 \\
\end{array}$ & $\begin{array}{r}100 . \\
2 \\
\end{array}$ & $\begin{array}{r}17.80 \\
00 \\
\end{array}$ & 2.5 & 0.5662 & 3.3 & $\begin{array}{r}0.073 \\
1 \\
\end{array}$ & $\begin{array}{r}2 . \\
1 \\
\end{array}$ & $\begin{array}{r}0.6 \\
3 \\
\end{array}$ & 454.8 & 9.1 & 455.6 & 12.0 & 459.5 & 55.9 & 454.8 & 9.1 & NA \\
\hline $\begin{array}{l}\text { BU0754- } \\
89\end{array}$ & $\begin{array}{r}161 \\
0 \\
\end{array}$ & $\begin{array}{r}18729 \\
0 \\
\end{array}$ & 19.3 & $\begin{array}{r}17.80 \\
32 \\
\end{array}$ & 1.3 & 0.5666 & 2.2 & $\begin{array}{r}0.073 \\
2 \\
\end{array}$ & $\begin{array}{l}1 . \\
8\end{array}$ & $\begin{array}{r}0.8 \\
1 \\
\end{array}$ & 455.1 & 7.8 & 455.8 & 8.0 & 459.1 & 28.4 & 455.1 & 7.8 & NA \\
\hline $\begin{array}{l}\text { BU0754- } \\
88\end{array}$ & 765 & $\begin{array}{r}10175 \\
0 \\
\end{array}$ & 3.7 & $\begin{array}{r}17.43 \\
83 \\
\end{array}$ & 2.1 & 0.5791 & 2.3 & $\begin{array}{r}0.073 \\
2 \\
\end{array}$ & $\begin{array}{r}1 . \\
0\end{array}$ & $\begin{array}{r}0.4 \\
4 \\
\end{array}$ & 455.7 & 4.5 & 463.9 & 8.6 & 504.8 & 45.6 & 455.7 & 4.5 & NA \\
\hline $\begin{array}{l}\text { BU0754- } \\
18\end{array}$ & $\begin{array}{r}170 \\
1 \\
\end{array}$ & $\begin{array}{r}15074 \\
5 \\
\end{array}$ & 4.7 & $\begin{array}{r}17.42 \\
88 \\
\end{array}$ & 2.0 & 0.5819 & 3.4 & $\begin{array}{r}0.073 \\
6 \\
\end{array}$ & $\begin{array}{l}2 . \\
7 \\
\end{array}$ & $\begin{array}{r}0.8 \\
1 \\
\end{array}$ & 457.5 & 12.0 & 465.7 & 12.6 & 506.0 & 43.8 & 457.5 & 12.0 & NA \\
\hline $\begin{array}{l}\text { BU0754- } \\
90\end{array}$ & $\begin{array}{r}118 \\
8 \\
\end{array}$ & $\begin{array}{r}15026 \\
0 \\
\end{array}$ & 1.5 & $\begin{array}{r}17.13 \\
81 \\
\end{array}$ & 2.3 & 0.5920 & 4.1 & $\begin{array}{r}0.073 \\
6 \\
\end{array}$ & $\begin{array}{r}3 . \\
4\end{array}$ & $\begin{array}{r}0.8 \\
3 \\
\end{array}$ & 457.7 & 15.0 & 472.1 & 15.4 & 542.9 & 49.4 & 457.7 & 15.0 & NA \\
\hline $\begin{array}{l}\text { BU0754- } \\
30\end{array}$ & $\begin{array}{r}371 \\
1 \\
\end{array}$ & $\begin{array}{r}22111 \\
5 \\
\end{array}$ & 1.0 & $\begin{array}{r}17.57 \\
27 \\
\end{array}$ & 2.0 & 0.5774 & 3.1 & $\begin{array}{r}0.073 \\
6 \\
\end{array}$ & $\begin{array}{r}2 . \\
3 \\
\end{array}$ & $\begin{array}{r}0.7 \\
5 \\
\end{array}$ & 457.8 & 10.3 & 462.8 & 11.4 & 487.9 & 44.6 & 457.8 & 10.3 & NA \\
\hline $\begin{array}{l}\text { BU0754- } \\
52\end{array}$ & $\begin{array}{r}300 \\
1 \\
\end{array}$ & $\begin{array}{r}32549 \\
5 \\
\end{array}$ & 56.7 & $\begin{array}{r}17.70 \\
60 \\
\end{array}$ & 2.3 & 0.5731 & 2.5 & $\begin{array}{r}0.073 \\
6 \\
\end{array}$ & $\begin{array}{r}1 . \\
0\end{array}$ & $\begin{array}{r}0.4 \\
1 \\
\end{array}$ & 457.8 & 4.4 & 460.0 & 9.1 & 471.2 & 49.8 & 457.8 & 4.4 & NA \\
\hline $\begin{array}{l}\text { BU0754- } \\
60\end{array}$ & 863 & $\begin{array}{r}29938 \\
0 \\
\end{array}$ & 52.1 & $\begin{array}{r}17.68 \\
48 \\
\end{array}$ & 2.6 & 0.5740 & 4.0 & $\begin{array}{r}0.073 \\
6 \\
\end{array}$ & $\begin{array}{r}2 . \\
9 \\
\end{array}$ & $\begin{array}{r}0.7 \\
4 \\
\end{array}$ & 458.0 & 13.0 & 460.6 & 14.6 & 473.8 & 58.4 & 458.0 & 13.0 & NA \\
\hline $\begin{array}{l}\text { BU0754- } \\
97\end{array}$ & 601 & $\begin{array}{r}12632 \\
5 \\
\end{array}$ & 14.9 & $\begin{array}{r}17.04 \\
43 \\
\end{array}$ & 3.6 & 0.6014 & 4.2 & $\begin{array}{r}0.074 \\
3 \\
\end{array}$ & $\begin{array}{r}2 . \\
1\end{array}$ & $\begin{array}{r}0.5 \\
0 \\
\end{array}$ & 462.3 & 9.3 & 478.2 & 16.0 & 554.9 & 79.4 & 462.3 & 9.3 & NA \\
\hline $\begin{array}{l}\text { BU0754- } \\
67\end{array}$ & 668 & 52815 & 1.3 & $\begin{array}{r}17.41 \\
09 \\
\end{array}$ & 2.3 & 0.5888 & 3.0 & $\begin{array}{r}0.074 \\
4 \\
\end{array}$ & $\begin{array}{r}1 . \\
8\end{array}$ & $\begin{array}{r}0.6 \\
2 \\
\end{array}$ & 462.4 & 8.1 & 470.1 & 11.1 & 508.3 & 51.3 & 462.4 & 8.1 & NA \\
\hline $\begin{array}{l}\text { BU0754- } \\
29\end{array}$ & $\begin{array}{r}205 \\
9 \\
\end{array}$ & $\begin{array}{r}15469 \\
5 \\
\end{array}$ & 2.2 & $\begin{array}{r}17.72 \\
09 \\
\end{array}$ & 1.0 & 0.5791 & 2.4 & $\begin{array}{r}0.074 \\
4 \\
\end{array}$ & $\begin{array}{r}2 . \\
2 \\
\end{array}$ & $\begin{array}{r}0.9 \\
1 \\
\end{array}$ & 462.8 & 9.6 & 463.9 & 8.9 & 469.3 & 22.1 & 462.8 & 9.6 & NA \\
\hline $\begin{array}{l}\text { BU0754- } \\
95\end{array}$ & 994 & $\begin{array}{r}12428 \\
0 \\
\end{array}$ & 0.8 & $\begin{array}{r}17.50 \\
48 \\
\end{array}$ & 2.5 & 0.5873 & 3.7 & $\begin{array}{r}0.074 \\
6 \\
\end{array}$ & $\begin{array}{r}2 . \\
8 \\
\end{array}$ & $\begin{array}{r}0.7 \\
5 \\
\end{array}$ & 463.6 & 12.3 & 469.1 & 13.9 & 496.4 & 54.4 & 463.6 & 12.3 & NA \\
\hline $\begin{array}{l}\text { BU0754- } \\
81\end{array}$ & 751 & $\begin{array}{r}15644 \\
0 \\
\end{array}$ & 3.6 & $\begin{array}{r}17.23 \\
59 \\
\end{array}$ & 3.7 & 0.6047 & 3.8 & $\begin{array}{r}0.075 \\
6 \\
\end{array}$ & $\begin{array}{r}1 . \\
0\end{array}$ & $\begin{array}{r}0.2 \\
6 \\
\end{array}$ & 469.8 & 4.5 & 480.2 & 14.5 & 530.4 & 80.2 & 469.8 & 4.5 & NA \\
\hline $\begin{array}{l}\text { BU0754- } \\
80\end{array}$ & 549 & 93240 & 14.7 & $\begin{array}{r}16.52 \\
50 \\
\end{array}$ & 3.6 & 0.6309 & 4.1 & $\begin{array}{r}0.075 \\
6 \\
\end{array}$ & $\begin{array}{r}2 . \\
1 \\
\end{array}$ & $\begin{array}{r}0.5 \\
1 \\
\end{array}$ & 469.9 & 9.5 & 496.7 & 16.2 & 622.0 & 76.6 & 469.9 & 9.5 & NA \\
\hline $\begin{array}{l}\text { BU0754- } \\
75\end{array}$ & $\begin{array}{r}167 \\
0 \\
\end{array}$ & $\begin{array}{r}20759 \\
5 \\
\end{array}$ & 7.4 & $\begin{array}{r}17.63 \\
70 \\
\end{array}$ & 1.8 & 0.5913 & 2.9 & $\begin{array}{r}0.075 \\
6 \\
\end{array}$ & $\begin{array}{r}2 . \\
4 \\
\end{array}$ & $\begin{array}{r}0.8 \\
0 \\
\end{array}$ & 470.0 & 10.7 & 471.7 & 11.1 & 479.8 & 38.9 & 470.0 & 10.7 & NA \\
\hline $\begin{array}{l}\text { BU0754- } \\
10\end{array}$ & $\begin{array}{r}375 \\
6 \\
\end{array}$ & $\begin{array}{r}10235 \\
0 \\
\end{array}$ & 2.4 & $\begin{array}{r}17.50 \\
01 \\
\end{array}$ & 1.0 & 0.5962 & 2.3 & $\begin{array}{r}0.075 \\
7 \\
\end{array}$ & $\begin{array}{r}2 . \\
1\end{array}$ & $\begin{array}{r}0.9 \\
0 \\
\end{array}$ & 470.2 & 9.4 & 474.8 & 8.7 & 497.0 & 22.1 & 470.2 & 9.4 & NA \\
\hline $\begin{array}{l}\text { BU0754- } \\
53\end{array}$ & 136 & 13615 & 1.0 & $\begin{array}{r}15.13 \\
95 \\
\end{array}$ & 7.8 & 0.6900 & 7.9 & $\begin{array}{r}0.075 \\
8 \\
\end{array}$ & $\begin{array}{c}1 . \\
1 \\
\end{array}$ & $\begin{array}{r}0.1 \\
4 \\
\end{array}$ & 470.8 & 4.9 & 532.8 & 32.7 & 808.0 & $\begin{array}{r}163 . \\
6 \\
\end{array}$ & 470.8 & 4.9 & NA \\
\hline BU0754-4 & 183 & 13410 & 1.9 & 17.41 & 1.8 & 0.6030 & 2.3 & 0.076 & 1. & 0.6 & 473.1 & 6.8 & 479.2 & 9.0 & 508.1 & 40.0 & 473.1 & 6.8 & NA \\
\hline
\end{tabular}




\begin{tabular}{|c|c|c|c|c|c|c|c|c|c|c|c|c|c|c|c|c|c|c|c|}
\hline & 0 & 0 & & 18 & & & & 2 & 5 & 3 & & & & & & & & & \\
\hline $\begin{array}{l}\text { BU0754- } \\
78\end{array}$ & $\begin{array}{r}114 \\
5\end{array}$ & $\begin{array}{r}27745 \\
5\end{array}$ & 47.8 & $\begin{array}{r}17.63 \\
95\end{array}$ & 3.9 & 0.5967 & 4.0 & $\begin{array}{r}0.076 \\
3\end{array}$ & $\begin{array}{r}1 . \\
0\end{array}$ & $\begin{array}{r}0.2 \\
5\end{array}$ & 474.3 & 4.6 & 475.2 & 15.3 & 479.5 & 86.2 & 474.3 & 4.6 & NA \\
\hline $\begin{array}{l}\text { BU0754- } \\
17\end{array}$ & 168 & 17920 & 1.1 & $\begin{array}{r}16.88 \\
97\end{array}$ & 1.7 & 0.6251 & 3.0 & $\begin{array}{r}0.076 \\
6\end{array}$ & $\begin{array}{r}2 . \\
5\end{array}$ & $\begin{array}{r}0.8 \\
2\end{array}$ & 475.6 & 11.2 & 493.0 & 11.7 & 574.7 & 37.6 & 475.6 & 11.2 & NA \\
\hline $\begin{array}{l}\text { BU0754- } \\
92\end{array}$ & $\begin{array}{r}266 \\
6\end{array}$ & $\begin{array}{r}33162 \\
5\end{array}$ & 21.9 & $\begin{array}{r}17.80 \\
16\end{array}$ & 2.6 & 0.5932 & 3.1 & $\begin{array}{r}0.076 \\
6\end{array}$ & $\begin{array}{r}1 . \\
6\end{array}$ & $\begin{array}{r}0.5 \\
2\end{array}$ & 475.7 & 7.4 & 472.9 & 11.6 & 459.3 & 58.1 & 475.7 & 7.4 & NA \\
\hline BU0754-8 & $\begin{array}{r}430 \\
5\end{array}$ & $\begin{array}{r}49647 \\
5\end{array}$ & 33.2 & $\begin{array}{r}17.67 \\
66\end{array}$ & 2.0 & 0.5993 & 3.9 & $\begin{array}{r}0.076 \\
8\end{array}$ & $\begin{array}{r}3 . \\
4\end{array}$ & $\begin{array}{r}0.8 \\
6\end{array}$ & 477.2 & 15.6 & 476.8 & 15.0 & 474.9 & 44.2 & 477.2 & 15.6 & NA \\
\hline $\begin{array}{l}\text { BU0754- } \\
21\end{array}$ & $\begin{array}{r}174 \\
5 \\
\end{array}$ & $\begin{array}{r}18538 \\
5 \\
\end{array}$ & 5.6 & $\begin{array}{r}17.54 \\
15 \\
\end{array}$ & 2.0 & 0.6040 & 2.4 & $\begin{array}{r}0.076 \\
8 \\
\end{array}$ & $\begin{array}{r}1 . \\
4\end{array}$ & $\begin{array}{r}0.5 \\
6 \\
\end{array}$ & 477.3 & 6.3 & 479.8 & 9.3 & 491.8 & 44.3 & 477.3 & 6.3 & NA \\
\hline $\begin{array}{l}\text { BU0754- } \\
59\end{array}$ & $\begin{array}{r}313 \\
3\end{array}$ & $\begin{array}{r}35062 \\
0\end{array}$ & 68.1 & $\begin{array}{r}17.35 \\
66\end{array}$ & 4.2 & 0.6144 & 4.9 & $\begin{array}{r}0.077 \\
3\end{array}$ & $\begin{array}{r}2 . \\
5\end{array}$ & $\begin{array}{r}0.5 \\
0\end{array}$ & 480.2 & 11.4 & 486.3 & 19.0 & 515.1 & 93.2 & 480.2 & 11.4 & NA \\
\hline $\begin{array}{l}\text { BU0754- } \\
63\end{array}$ & 977 & $\begin{array}{r}17550 \\
5\end{array}$ & 14.7 & $\begin{array}{r}17.19 \\
50\end{array}$ & 4.0 & 0.6216 & 5.2 & $\begin{array}{r}0.077 \\
5\end{array}$ & $\begin{array}{r}3 . \\
4\end{array}$ & $\begin{array}{r}0.6 \\
5\end{array}$ & 481.3 & 15.8 & 490.8 & 20.4 & 535.6 & 87.4 & 481.3 & 15.8 & NA \\
\hline $\begin{array}{l}\text { BU0754- } \\
23\end{array}$ & $\begin{array}{r}207 \\
2\end{array}$ & $\begin{array}{r}17968 \\
5\end{array}$ & 22.2 & $\begin{array}{r}17.48 \\
80\end{array}$ & 1.2 & 0.6199 & 3.0 & $\begin{array}{r}0.078 \\
6\end{array}$ & $\begin{array}{l}2 . \\
7\end{array}$ & $\begin{array}{r}0.9 \\
1\end{array}$ & 487.9 & 12.8 & 489.8 & 11.6 & 498.5 & 26.9 & 487.9 & 12.8 & NA \\
\hline $\begin{array}{l}\text { BU0754- } \\
34\end{array}$ & $\begin{array}{r}171 \\
9\end{array}$ & $\begin{array}{r}17668 \\
0\end{array}$ & 1.0 & $\begin{array}{r}17.46 \\
88\end{array}$ & 7.4 & 0.6207 & 7.5 & $\begin{array}{r}0.078 \\
6\end{array}$ & $\begin{array}{r}1 . \\
2\end{array}$ & $\begin{array}{r}0.1 \\
5\end{array}$ & 488.0 & 5.4 & 490.3 & 29.2 & 501.0 & $\begin{array}{r}163 . \\
6\end{array}$ & 488.0 & 5.4 & NA \\
\hline $\begin{array}{l}\text { BU0754- } \\
77\end{array}$ & 832 & $\begin{array}{r}18853 \\
5\end{array}$ & 41.7 & $\begin{array}{r}17.07 \\
66\end{array}$ & 6.6 & 0.6368 & 7.8 & $\begin{array}{r}0.078 \\
9\end{array}$ & $\begin{array}{r}4 . \\
2\end{array}$ & $\begin{array}{r}0.5 \\
3\end{array}$ & 489.4 & 19.7 & 500.3 & 30.9 & 550.7 & $\begin{array}{r}144 . \\
4\end{array}$ & 489.4 & 19.7 & NA \\
\hline $\begin{array}{l}\text { BU0754- } \\
87\end{array}$ & 393 & 65485 & 5.2 & $\begin{array}{r}17.35 \\
65\end{array}$ & 2.1 & 0.6273 & 2.5 & $\begin{array}{r}0.079 \\
0\end{array}$ & $\begin{array}{l}1 . \\
3\end{array}$ & $\begin{array}{r}0.5 \\
5\end{array}$ & 490.0 & 6.3 & 494.4 & 9.6 & 515.1 & 45.3 & 490.0 & 6.3 & NA \\
\hline $\begin{array}{l}\text { BU0754- } \\
57\end{array}$ & 598 & 85310 & 5.0 & $\begin{array}{r}16.81 \\
52\end{array}$ & 4.7 & 0.6522 & 4.8 & $\begin{array}{r}0.079 \\
5\end{array}$ & $\begin{array}{l}1 . \\
0\end{array}$ & $\begin{array}{r}0.2 \\
1\end{array}$ & 493.3 & 4.7 & 509.8 & 19.2 & 584.3 & $\begin{array}{r}101 . \\
7\end{array}$ & 493.3 & 4.7 & NA \\
\hline $\begin{array}{l}\text { BU0754- } \\
15\end{array}$ & $\begin{array}{r}438 \\
1\end{array}$ & $\begin{array}{r}40060 \\
0\end{array}$ & 44.9 & $\begin{array}{r}17.48 \\
77\end{array}$ & 1.6 & 0.6272 & 1.9 & $\begin{array}{r}0.079 \\
5\end{array}$ & $\begin{array}{r}1 . \\
2\end{array}$ & $\begin{array}{r}0.5 \\
9\end{array}$ & 493.4 & 5.5 & 494.3 & 7.6 & 498.6 & 34.6 & 493.4 & 5.5 & NA \\
\hline $\begin{array}{l}\text { BU0754- } \\
76\end{array}$ & 682 & $\begin{array}{r}11816 \\
0\end{array}$ & 1.5 & $\begin{array}{r}17.27 \\
52\end{array}$ & 5.7 & 0.6458 & 5.8 & $\begin{array}{r}0.080 \\
9\end{array}$ & $\begin{array}{l}1 . \\
0\end{array}$ & $\begin{array}{r}0.1 \\
7\end{array}$ & 501.6 & 4.8 & 505.9 & 22.9 & 525.4 & $\begin{array}{r}124 . \\
4\end{array}$ & 501.6 & 4.8 & 95.5 \\
\hline BU0754-6 & $\begin{array}{r}154 \\
4\end{array}$ & $\begin{array}{r}16765 \\
0\end{array}$ & 1.5 & $\begin{array}{r}17.57 \\
57\end{array}$ & 1.7 & 0.6377 & 2.0 & $\begin{array}{r}0.081 \\
3\end{array}$ & $\begin{array}{l}1 . \\
0\end{array}$ & $\begin{array}{r}0.5 \\
0\end{array}$ & 503.8 & 4.8 & 500.9 & 7.9 & 487.5 & 38.0 & 503.8 & 4.8 & $\begin{array}{r}103 . \\
3\end{array}$ \\
\hline BU0754-2 & 414 & 43325 & 0.8 & $\begin{array}{r}17.35 \\
38\end{array}$ & 1.4 & 0.6479 & 2.5 & $\begin{array}{r}0.081 \\
5\end{array}$ & $\begin{array}{r}2 . \\
1\end{array}$ & $\begin{array}{r}0.8 \\
2\end{array}$ & 505.4 & 10.1 & 507.2 & 10.1 & 515.5 & 31.4 & 505.4 & 10.1 & 98.0 \\
\hline $\begin{array}{l}\text { BU0754- } \\
96\end{array}$ & 469 & $\begin{array}{r}12589 \\
5\end{array}$ & 6.5 & $\begin{array}{r}16.83 \\
92\end{array}$ & 3.2 & 0.6840 & 3.3 & $\begin{array}{r}0.083 \\
5\end{array}$ & $\begin{array}{r}1 . \\
0\end{array}$ & $\begin{array}{r}0.3 \\
0\end{array}$ & 517.2 & 5.0 & 529.2 & 13.6 & 581.2 & 68.4 & 517.2 & 5.0 & 89.0 \\
\hline $\begin{array}{l}\text { BU0754- } \\
22\end{array}$ & $\begin{array}{r}134 \\
7\end{array}$ & $\begin{array}{r}13842 \\
0\end{array}$ & 10.5 & $\begin{array}{r}17.31 \\
82\end{array}$ & 4.4 & 0.6810 & 6.2 & $\begin{array}{r}0.085 \\
5\end{array}$ & $\begin{array}{r}4 . \\
4\end{array}$ & $\begin{array}{r}0.7 \\
0\end{array}$ & 529.1 & 22.1 & 527.4 & 25.5 & 520.0 & 97.0 & 529.1 & 22.1 & $\begin{array}{r}101 . \\
7\end{array}$ \\
\hline $\begin{array}{l}\text { BU0754- } \\
99\end{array}$ & 259 & 71005 & 1.5 & $\begin{array}{r}16.95 \\
15\end{array}$ & 4.3 & 0.7009 & 4.8 & $\begin{array}{r}0.086 \\
2\end{array}$ & $\begin{array}{r}2 . \\
0\end{array}$ & $\begin{array}{r}0.4 \\
2\end{array}$ & 532.8 & 10.3 & 539.3 & 20.0 & 566.8 & 94.5 & 532.8 & 10.3 & 94.0 \\
\hline $\begin{array}{l}\text { BU0754- } \\
40\end{array}$ & $\begin{array}{r}729 \\
7\end{array}$ & $\begin{array}{r}15908 \\
5\end{array}$ & 46.5 & $\begin{array}{r}13.55 \\
83\end{array}$ & 2.0 & 0.9062 & 2.5 & $\begin{array}{r}0.089 \\
1\end{array}$ & $\begin{array}{r}1 . \\
5\end{array}$ & $\begin{array}{r}0.6 \\
0\end{array}$ & 550.3 & 8.0 & 655.0 & 12.3 & $\begin{array}{r}1034 . \\
8\end{array}$ & 41.2 & 550.3 & 8.0 & 53.2 \\
\hline $\begin{array}{l}\text { BU0754- } \\
91\end{array}$ & 696 & 85825 & 6.9 & $\begin{array}{r}12.25 \\
11\end{array}$ & 3.6 & 1.0481 & 4.0 & $\begin{array}{r}0.093 \\
1\end{array}$ & $\begin{array}{r}1 . \\
8\end{array}$ & $\begin{array}{r}0.4 \\
5\end{array}$ & 574.0 & 10.1 & 728.0 & 21.0 & $\begin{array}{r}1236 . \\
5\end{array}$ & 70.6 & 574.0 & 10.1 & 46.4 \\
\hline $\begin{array}{l}\text { BU0754- } \\
45\end{array}$ & $\begin{array}{r}158 \\
2\end{array}$ & $\begin{array}{r}17438 \\
5\end{array}$ & 15.1 & $\begin{array}{r}16.63 \\
64\end{array}$ & 1.0 & 0.8149 & 1.4 & $\begin{array}{r}0.098 \\
3\end{array}$ & $\begin{array}{l}1 . \\
0\end{array}$ & $\begin{array}{r}0.7 \\
1\end{array}$ & 604.6 & 5.8 & 605.2 & 6.4 & 607.5 & 21.6 & 604.6 & 5.8 & 99.5 \\
\hline $\begin{array}{l}\text { BU0754- } \\
27\end{array}$ & 633 & 40070 & 3.7 & $\begin{array}{r}14.56 \\
04\end{array}$ & 2.0 & 0.9750 & 3.7 & $\begin{array}{r}0.103 \\
0\end{array}$ & $\begin{array}{r}3 . \\
1\end{array}$ & $\begin{array}{r}0.8 \\
5\end{array}$ & 631.7 & 18.9 & 691.0 & 18.5 & 889.1 & 40.4 & 631.7 & 18.9 & 71.1 \\
\hline $\begin{array}{l}\text { BU0754- } \\
46\end{array}$ & 448 & 75495 & 4.9 & $\begin{array}{r}15.91 \\
38\end{array}$ & 2.6 & 0.9636 & 2.8 & $\begin{array}{r}0.111 \\
2\end{array}$ & $\begin{array}{l}1 . \\
0\end{array}$ & $\begin{array}{r}0.3 \\
6\end{array}$ & 679.8 & 6.5 & 685.2 & 13.8 & 702.8 & 54.9 & 679.8 & 6.5 & 96.7 \\
\hline
\end{tabular}




\begin{tabular}{|c|c|c|c|c|c|c|c|c|c|c|c|c|c|c|c|c|c|c|c|}
\hline $\begin{array}{l}\text { BU0754- } \\
43\end{array}$ & $\begin{array}{r}122 \\
2 \\
\end{array}$ & $\begin{array}{r}20912 \\
0 \\
\end{array}$ & 4.0 & $\begin{array}{r}14.75 \\
26 \\
\end{array}$ & 1.5 & 1.1460 & 2.1 & $\begin{array}{r}0.122 \\
6 \\
\end{array}$ & $\begin{array}{r}1 . \\
5\end{array}$ & $\begin{array}{r}0.7 \\
0 \\
\end{array}$ & 745.6 & 10.2 & 775.4 & 11.2 & 862.0 & 30.7 & 745.6 & 10.2 & 86.5 \\
\hline $\begin{array}{l}\text { BU0754- } \\
86\end{array}$ & 623 & $\begin{array}{r}22134 \\
0\end{array}$ & 6.1 & $\begin{array}{r}14.25 \\
90\end{array}$ & 1.4 & 1.2338 & 2.5 & $\begin{array}{r}0.127 \\
6\end{array}$ & $\begin{array}{r}2 . \\
1\end{array}$ & $\begin{array}{r}0.8 \\
3\end{array}$ & 774.1 & 15.0 & 816.1 & 13.9 & 932.2 & 28.5 & 774.1 & 15.0 & 83.0 \\
\hline $\begin{array}{l}\text { BU0754- } \\
28\end{array}$ & 186 & 34315 & 0.5 & $\begin{array}{r}15.02 \\
44 \\
\end{array}$ & 2.2 & 1.2053 & 2.6 & $\begin{array}{r}0.131 \\
3 \\
\end{array}$ & $\begin{array}{r}1 . \\
5 \\
\end{array}$ & $\begin{array}{r}0.5 \\
6\end{array}$ & 795.5 & 10.9 & 803.0 & 14.4 & 824.0 & 45.1 & 795.5 & 10.9 & 96.5 \\
\hline $\begin{array}{l}\text { BU0754- } \\
16\end{array}$ & 383 & 60000 & 1.3 & $\begin{array}{r}14.74 \\
13 \\
\end{array}$ & 2.0 & 1.2579 & 3.6 & $\begin{array}{r}0.134 \\
5 \\
\end{array}$ & $\begin{array}{r}3 . \\
0\end{array}$ & $\begin{array}{r}0.8 \\
4 \\
\end{array}$ & 813.4 & 23.2 & 827.0 & 20.4 & 863.5 & 40.5 & 813.4 & 23.2 & 94.2 \\
\hline $\begin{array}{l}\text { BU0754- } \\
24\end{array}$ & 125 & 17360 & 0.7 & $\begin{array}{r}14.60 \\
75 \\
\end{array}$ & 4.6 & 1.2945 & 4.8 & $\begin{array}{r}0.137 \\
1 \\
\end{array}$ & $\begin{array}{r}. \\
4 \\
\end{array}$ & $\begin{array}{r}0.3 \\
0 \\
\end{array}$ & 828.5 & 11.2 & 843.3 & 27.6 & 882.4 & 95.3 & 828.5 & 11.2 & 93.9 \\
\hline $\begin{array}{l}\text { BU0754- } \\
72\end{array}$ & 836 & $\begin{array}{r}16387 \\
5 \\
\end{array}$ & 0.6 & $\begin{array}{r}14.64 \\
31 \\
\end{array}$ & 2.6 & 1.2920 & 3.2 & $\begin{array}{r}0.137 \\
2 \\
\end{array}$ & $\begin{array}{c}1 . \\
9\end{array}$ & $\begin{array}{r}0.6 \\
0 \\
\end{array}$ & 828.9 & 14.9 & 842.2 & 18.4 & 877.4 & 53.2 & 828.9 & 14.9 & 94.5 \\
\hline $\begin{array}{l}\text { BU0754- } \\
74\end{array}$ & 158 & 38905 & 0.9 & $\begin{array}{r}14.50 \\
88\end{array}$ & 1.9 & 1.3423 & 2.2 & $\begin{array}{r}0.141 \\
2\end{array}$ & $\begin{array}{r}1 . \\
0\end{array}$ & $\begin{array}{r}0.4 \\
6\end{array}$ & 851.7 & 8.0 & 864.2 & 12.6 & 896.4 & 39.6 & 851.7 & 8.0 & 95.0 \\
\hline BU0754-1 & $\begin{array}{r}104 \\
0 \\
\end{array}$ & $\begin{array}{r}12947 \\
0 \\
\end{array}$ & 2.4 & $\begin{array}{r}13.84 \\
85 \\
\end{array}$ & 2.3 & 1.4077 & 3.7 & $\begin{array}{r}0.141 \\
4 \\
\end{array}$ & $\begin{array}{r}2 . \\
9 \\
\end{array}$ & $\begin{array}{r}0.7 \\
9 \\
\end{array}$ & 852.5 & 23.5 & 892.2 & 22.2 & 991.8 & 47.0 & 852.5 & 23.5 & 86.0 \\
\hline $\begin{array}{l}\text { BU0754- } \\
64\end{array}$ & 117 & 44640 & 1.1 & $\begin{array}{r}14.38 \\
22 \\
\end{array}$ & 4.8 & 1.4097 & 5.9 & $\begin{array}{r}0.147 \\
0 \\
\end{array}$ & $\begin{array}{c}3 . \\
5\end{array}$ & $\begin{array}{r}0.5 \\
9 \\
\end{array}$ & 884.4 & 29.0 & 893.0 & 35.1 & 914.5 & 97.8 & 884.4 & 29.0 & 96.7 \\
\hline $\begin{array}{l}\text { BU0754- } \\
55\end{array}$ & 224 & 83850 & 1.8 & $\begin{array}{r}14.75 \\
53 \\
\end{array}$ & 2.1 & 1.3842 & 2.8 & $\begin{array}{r}0.148 \\
1 \\
\end{array}$ & $\begin{array}{r}1 . \\
9\end{array}$ & $\begin{array}{r}0.6 \\
7 \\
\end{array}$ & 890.5 & 15.5 & 882.2 & 16.3 & 861.6 & 42.5 & 890.5 & 15.5 & $\begin{array}{r}103 . \\
4 \\
\end{array}$ \\
\hline $\begin{array}{l}\text { BU0754- } \\
68\end{array}$ & 486 & $\begin{array}{r}14760 \\
0\end{array}$ & 1.8 & $\begin{array}{r}14.15 \\
03\end{array}$ & 1.3 & 1.4448 & 1.7 & $\begin{array}{r}0.148 \\
3\end{array}$ & $\begin{array}{r}1 . \\
0\end{array}$ & $\begin{array}{r}0.6 \\
0\end{array}$ & 891.3 & 8.3 & 907.7 & 9.9 & 947.9 & 27.0 & 891.3 & 8.3 & 94.0 \\
\hline $\begin{array}{l}\text { BU0754- } \\
33\end{array}$ & $\begin{array}{r}199 \\
2 \\
\end{array}$ & $\begin{array}{r}32754 \\
0\end{array}$ & 5.7 & $\begin{array}{r}14.44 \\
67\end{array}$ & 1.1 & 1.4692 & 1.5 & $\begin{array}{r}0.153 \\
9\end{array}$ & $\begin{array}{r}1 . \\
0\end{array}$ & $\begin{array}{r}0.6 \\
7\end{array}$ & 923.0 & 8.6 & 917.8 & 9.0 & 905.3 & 22.9 & 923.0 & 8.6 & $\begin{array}{r}102 . \\
0\end{array}$ \\
\hline $\begin{array}{l}\text { BU0754- } \\
11\end{array}$ & 487 & $\begin{array}{r}12864 \\
0 \\
\end{array}$ & 1.3 & $\begin{array}{r}14.14 \\
76 \\
\end{array}$ & 1.2 & 1.5281 & 1.5 & $\begin{array}{r}0.156 \\
8 \\
\end{array}$ & $\begin{array}{l}1 . \\
0 \\
\end{array}$ & $\begin{array}{r}0.6 \\
6 \\
\end{array}$ & 939.0 & 8.7 & 941.7 & 9.4 & 948.3 & 23.5 & 939.0 & 8.7 & 99.0 \\
\hline $\begin{array}{l}\text { BU0754- } \\
42\end{array}$ & 236 & 46775 & 1.4 & $\begin{array}{r}14.09 \\
88 \\
\end{array}$ & 3.2 & 1.6134 & 3.4 & $\begin{array}{r}0.165 \\
0 \\
\end{array}$ & $\begin{array}{r}1 . \\
2 \\
\end{array}$ & $\begin{array}{r}0.3 \\
4 \\
\end{array}$ & 984.4 & 10.8 & 975.4 & 21.6 & 955.3 & 66.1 & 984.4 & 10.8 & $\begin{array}{r}103 . \\
0 \\
\end{array}$ \\
\hline $\begin{array}{l}\text { BU0754- } \\
39\end{array}$ & 311 & 81425 & 2.3 & $\begin{array}{r}13.72 \\
57 \\
\end{array}$ & 1.8 & 1.6071 & 2.1 & $\begin{array}{r}0.160 \\
0 \\
\end{array}$ & $\begin{array}{r}1 . \\
1\end{array}$ & $\begin{array}{r}0.5 \\
2 \\
\end{array}$ & 956.7 & 9.7 & 973.0 & 13.0 & $\begin{array}{r}1009 . \\
9 \\
\end{array}$ & 35.9 & 1009.9 & 35.9 & 94.7 \\
\hline $\begin{array}{l}\text { BU0754- } \\
51\end{array}$ & 797 & $\begin{array}{r}12084 \\
0\end{array}$ & 4.5 & $\begin{array}{r}13.32 \\
97\end{array}$ & 2.1 & 1.7500 & 2.3 & $\begin{array}{r}0.169 \\
2\end{array}$ & $\begin{array}{r}1 . \\
0\end{array}$ & $\begin{array}{r}0.4 \\
3\end{array}$ & $\begin{array}{r}1007 . \\
6\end{array}$ & 9.3 & 1027.2 & 15.0 & $\begin{array}{r}1069 . \\
0\end{array}$ & 42.0 & 1069.0 & 42.0 & 94.3 \\
\hline $\begin{array}{l}\text { BU0754- } \\
93\end{array}$ & 516 & $\begin{array}{r}13882 \\
0 \\
\end{array}$ & 1.1 & $\begin{array}{r}12.47 \\
85 \\
\end{array}$ & 1.8 & 1.7321 & 3.5 & $\begin{array}{r}0.156 \\
8 \\
\end{array}$ & $\begin{array}{r}3 . \\
0\end{array}$ & $\begin{array}{r}0.8 \\
6 \\
\end{array}$ & 938.7 & 25.9 & 1020.5 & 22.2 & $\begin{array}{r}1200 . \\
4 \\
\end{array}$ & 34.7 & 1200.4 & 34.7 & 78.2 \\
\hline $\begin{array}{l}\text { BU0754- } \\
14\end{array}$ & $\begin{array}{r}236 \\
7\end{array}$ & $\begin{array}{r}31789 \\
5\end{array}$ & 6.1 & $\begin{array}{r}12.44 \\
56\end{array}$ & 2.0 & 1.6804 & 2.2 & $\begin{array}{r}0.151 \\
7\end{array}$ & $\begin{array}{l}1 . \\
0\end{array}$ & $\begin{array}{r}0.4 \\
5\end{array}$ & 910.4 & 8.5 & 1001.1 & 14.0 & $\begin{array}{r}1205 . \\
6\end{array}$ & 38.6 & 1205.6 & 38.6 & 75.5 \\
\hline $\begin{array}{l}\text { BU0754- } \\
20\end{array}$ & $\begin{array}{r}163 \\
0 \\
\end{array}$ & $\begin{array}{r}28051 \\
5 \\
\end{array}$ & 2.4 & $\begin{array}{r}11.83 \\
36 \\
\end{array}$ & 1.3 & 2.1658 & 5.0 & $\begin{array}{r}0.185 \\
9 \\
\end{array}$ & $\begin{array}{r}4 . \\
9 \\
\end{array}$ & $\begin{array}{r}0.9 \\
7\end{array}$ & $\begin{array}{r}1099 . \\
0\end{array}$ & 49.2 & 1170.1 & 35.0 & $\begin{array}{r}1304 . \\
2\end{array}$ & 25.2 & 1304.2 & 25.2 & 84.3 \\
\hline $\begin{array}{l}\text { BU0754- } \\
66\end{array}$ & 497 & $\begin{array}{r}19804 \\
0\end{array}$ & 3.4 & $\begin{array}{r}11.28 \\
05\end{array}$ & 2.2 & 2.0072 & 6.6 & $\begin{array}{r}0.164 \\
2\end{array}$ & $\begin{array}{r}6 . \\
2\end{array}$ & $\begin{array}{r}0.9 \\
4\end{array}$ & 980.2 & 56.7 & 1118.0 & 44.9 & $\begin{array}{r}1396 . \\
6\end{array}$ & 42.6 & 1396.6 & 42.6 & 70.2 \\
\hline $\begin{array}{l}\text { BU0754- } \\
83\end{array}$ & 96 & 66190 & 1.1 & $\begin{array}{r}11.09 \\
27\end{array}$ & 3.9 & 2.6851 & 4.0 & $\begin{array}{r}0.216 \\
0\end{array}$ & $\begin{array}{r}1 . \\
0\end{array}$ & $\begin{array}{r}0.2 \\
5\end{array}$ & $\begin{array}{r}1260 . \\
8\end{array}$ & 11.5 & 1324.4 & 29.4 & $\begin{array}{r}1428 . \\
7\end{array}$ & 73.5 & 1428.7 & 73.5 & 88.3 \\
\hline $\begin{array}{l}\text { BU0754- } \\
35\end{array}$ & $\begin{array}{r}133 \\
4\end{array}$ & 28520 & 1.9 & $\begin{array}{r}10.90 \\
68\end{array}$ & 3.0 & 1.1059 & 3.3 & $\begin{array}{r}0.087 \\
5\end{array}$ & $\begin{array}{r}1 . \\
4\end{array}$ & $\begin{array}{r}0.4 \\
2\end{array}$ & 540.6 & 7.2 & 756.2 & 17.6 & $\begin{array}{r}1460 . \\
9\end{array}$ & 56.8 & 1460.9 & 56.8 & 37.0 \\
\hline $\begin{array}{l}\text { BU0754- } \\
71\end{array}$ & 225 & 91795 & 0.8 & $\begin{array}{r}9.988 \\
7\end{array}$ & 1.3 & 3.9401 & 2.3 & $\begin{array}{r}0.285 \\
4\end{array}$ & $\begin{array}{r}2 . \\
0\end{array}$ & $\begin{array}{r}0.8 \\
4\end{array}$ & $\begin{array}{r}1618 . \\
7\end{array}$ & 27.9 & 1622.0 & 18.8 & $\begin{array}{r}1626 . \\
2\end{array}$ & 23.6 & 1626.2 & 23.6 & 99.5 \\
\hline BU0754-5 & 296 & $\begin{array}{r}11045 \\
0 \\
\end{array}$ & 0.8 & $\begin{array}{r}9.536 \\
7 \\
\end{array}$ & 1.1 & 4.3768 & 1.8 & $\begin{array}{r}0.302 \\
7 \\
\end{array}$ & $\begin{array}{r}1 . \\
4 \\
\end{array}$ & $\begin{array}{r}0.8 \\
0 \\
\end{array}$ & $\begin{array}{r}1704 . \\
8 \\
\end{array}$ & 21.4 & 1708.0 & 14.8 & $\begin{array}{r}1711 . \\
8 \\
\end{array}$ & 19.7 & 1711.8 & 19.7 & 99.6 \\
\hline BU0754- & 477 & 15753 & 5.4 & 9.327 & 1.1 & 3.1522 & 3.5 & 0.213 & 3. & 0.9 & 1246. & 37.7 & 1445.5 & 27.1 & 1752. & 20.9 & 1752.5 & 20.9 & 71.1 \\
\hline
\end{tabular}




\begin{tabular}{|c|c|c|c|c|c|c|c|c|c|c|c|c|c|c|c|c|c|c|c|}
\hline 94 & & 5 & & 5 & & & & 2 & 3 & 5 & 1 & & & & 5 & & & & \\
\hline $\begin{array}{l}\text { BU0754- } \\
100\end{array}$ & 246 & $\begin{array}{r}14931 \\
0 \\
\end{array}$ & 1.3 & $\begin{array}{r}9.071 \\
9 \\
\end{array}$ & 1.3 & 4.2381 & 2.7 & $\begin{array}{r}0.278 \\
8 \\
\end{array}$ & $\begin{array}{r}2 . \\
4 \\
\end{array}$ & $\begin{array}{r}0.8 \\
8 \\
\end{array}$ & $\begin{array}{r}1585 . \\
6 \\
\end{array}$ & 33.2 & 1681.4 & 22.1 & $\begin{array}{r}1803 . \\
2 \\
\end{array}$ & 23.6 & 1803.2 & 23.6 & 87.9 \\
\hline $\begin{array}{l}\text { BU0754- } \\
25\end{array}$ & 327 & $\begin{array}{r}16020 \\
0\end{array}$ & 1.2 & $\begin{array}{r}8.992 \\
8\end{array}$ & 1.0 & 4.8786 & 1.5 & $\begin{array}{r}0.318 \\
2\end{array}$ & $\begin{array}{c}1 . \\
1\end{array}$ & $\begin{array}{r}0.7 \\
3\end{array}$ & $\begin{array}{r}1780 . \\
9\end{array}$ & 16.8 & 1798.6 & 12.4 & $\begin{array}{r}1819 . \\
1\end{array}$ & 18.2 & 1819.1 & 18.2 & 97.9 \\
\hline $\begin{array}{l}\text { BU0754- } \\
13\end{array}$ & 75 & 52565 & 0.8 & $\begin{array}{r}5.975 \\
0 \\
\end{array}$ & 1.0 & $\begin{array}{r}11.111 \\
6 \\
\end{array}$ & 1.4 & $\begin{array}{r}0.481 \\
5 \\
\end{array}$ & $\begin{array}{r}1 . \\
0\end{array}$ & $\begin{array}{r}0.7 \\
1 \\
\end{array}$ & $\begin{array}{r}2533 . \\
9 \\
\end{array}$ & 21.0 & 2532.5 & 13.2 & $\begin{array}{r}2531 . \\
4\end{array}$ & 16.8 & 2531.4 & 16.8 & $\begin{array}{r}100 . \\
1\end{array}$ \\
\hline $\begin{array}{l}\text { BU0754- } \\
31\end{array}$ & 745 & $\begin{array}{r}35154 \\
5\end{array}$ & 1.1 & $\begin{array}{r}5.939 \\
2\end{array}$ & 1.2 & $\begin{array}{r}10.436 \\
5 \\
\end{array}$ & 1.6 & $\begin{array}{r}0.449 \\
6\end{array}$ & $\begin{array}{r}1 . \\
0\end{array}$ & $\begin{array}{r}0.6 \\
3\end{array}$ & $\begin{array}{r}2393 . \\
3\end{array}$ & 20.0 & 2474.3 & 14.7 & $\begin{array}{r}2541 . \\
5\end{array}$ & 20.6 & 2541.5 & 20.6 & 94.2 \\
\hline $\begin{array}{l}\text { BU0754- } \\
32\end{array}$ & $\begin{array}{r}114 \\
5 \\
\end{array}$ & $\begin{array}{r}44635 \\
5 \\
\end{array}$ & 3.6 & $\begin{array}{r}5.871 \\
8 \\
\end{array}$ & 1.3 & 9.2333 & 1.6 & $\begin{array}{r}0.393 \\
2 \\
\end{array}$ & $\begin{array}{r}1 . \\
0\end{array}$ & $\begin{array}{r}0.6 \\
4 \\
\end{array}$ & $\begin{array}{r}2137 . \\
7 \\
\end{array}$ & 18.7 & 2361.4 & 14.8 & $\begin{array}{r}2560 . \\
6 \\
\end{array}$ & 20.9 & 2560.6 & 20.9 & 83.5 \\
\hline $\begin{array}{l}\text { BU0755- } \\
15\end{array}$ & 166 & 15903 & 1.3 & $\begin{array}{r}13.94 \\
09 \\
\end{array}$ & 2.2 & 1.6308 & 3.0 & $\begin{array}{r}0.164 \\
9 \\
\end{array}$ & $\begin{array}{r}2 . \\
0\end{array}$ & $\begin{array}{r}0.6 \\
8\end{array}$ & 983.9 & 18.5 & 982.2 & 18.7 & 978.3 & 44.4 & 978.3 & 44.4 & $\begin{array}{r}100 . \\
6\end{array}$ \\
\hline $\begin{array}{l}\text { BU0755- } \\
12\end{array}$ & 419 & 32556 & 4.0 & $\begin{array}{r}13.73 \\
42\end{array}$ & 1.8 & 1.7098 & 2.8 & $\begin{array}{r}0.170 \\
3\end{array}$ & $\begin{array}{r}2 . \\
1\end{array}$ & $\begin{array}{r}0.7 \\
7\end{array}$ & $\begin{array}{r}1013 . \\
8\end{array}$ & 20.1 & 1012.2 & 17.8 & $\begin{array}{r}1008 . \\
7\end{array}$ & 35.9 & 1008.7 & 35.9 & $\begin{array}{r}100 . \\
5\end{array}$ \\
\hline $\begin{array}{l}\text { BU0755- } \\
30\end{array}$ & 703 & 38115 & 1.0 & $\begin{array}{r}13.72 \\
13 \\
\end{array}$ & 2.3 & 1.6337 & 2.9 & $\begin{array}{r}0.162 \\
6 \\
\end{array}$ & $\begin{array}{l}1 . \\
7\end{array}$ & $\begin{array}{r}0.5 \\
9 \\
\end{array}$ & 971.1 & 15.5 & 983.3 & 18.3 & $\begin{array}{r}1010 . \\
6\end{array}$ & 47.5 & 1010.6 & 47.5 & 96.1 \\
\hline $\begin{array}{l}\text { BU0755- } \\
52\end{array}$ & $\begin{array}{r}109 \\
2\end{array}$ & $\begin{array}{r}11028 \\
3\end{array}$ & 1.5 & $\begin{array}{r}13.69 \\
80\end{array}$ & 1.5 & 1.6635 & 1.9 & $\begin{array}{r}0.165 \\
3\end{array}$ & $\begin{array}{r}1 . \\
1\end{array}$ & $\begin{array}{r}0.5 \\
9\end{array}$ & 985.9 & 10.1 & 994.7 & 11.8 & $\begin{array}{r}1014 . \\
0\end{array}$ & 30.4 & 1014.0 & 30.4 & 97.2 \\
\hline $\begin{array}{l}\text { BU0755- } \\
60\end{array}$ & 871 & 36444 & 4.6 & $\begin{array}{r}13.52 \\
24\end{array}$ & 2.1 & 1.5833 & 2.9 & $\begin{array}{r}0.155 \\
3\end{array}$ & $\begin{array}{r}1 . \\
9\end{array}$ & $\begin{array}{r}0.6 \\
7\end{array}$ & 930.5 & 16.7 & 963.7 & 17.9 & $\begin{array}{r}1040 . \\
1\end{array}$ & 43.0 & 1040.1 & 43.0 & 89.5 \\
\hline $\begin{array}{l}\text { BU0755- } \\
13\end{array}$ & 453 & 48441 & 2.8 & $\begin{array}{r}13.47 \\
86\end{array}$ & 1.7 & 1.8191 & 3.0 & $\begin{array}{r}0.177 \\
8\end{array}$ & $\begin{array}{r}2 . \\
5\end{array}$ & $\begin{array}{r}0.8 \\
3\end{array}$ & $\begin{array}{r}1055 . \\
1\end{array}$ & 24.3 & 1052.4 & 19.8 & $\begin{array}{r}1046 . \\
7\end{array}$ & 34.3 & 1046.7 & 34.3 & $\begin{array}{r}100 . \\
8\end{array}$ \\
\hline $\begin{array}{l}\text { BU0755- } \\
61\end{array}$ & $\begin{array}{r}138 \\
8\end{array}$ & 34710 & 3.7 & $\begin{array}{r}13.10 \\
59\end{array}$ & 2.1 & 1.6841 & 3.9 & $\begin{array}{r}0.160 \\
1\end{array}$ & $\begin{array}{r}3 . \\
3\end{array}$ & $\begin{array}{r}0.8 \\
4\end{array}$ & 957.2 & 29.2 & 1002.5 & 24.8 & $\begin{array}{r}1103 . \\
0\end{array}$ & 41.8 & 1103.0 & 41.8 & 86.8 \\
\hline $\begin{array}{l}\text { BU0755- } \\
20\end{array}$ & 87 & 8781 & 1.1 & $\begin{array}{r}12.92 \\
37 \\
\end{array}$ & 3.9 & 1.8965 & 4.2 & $\begin{array}{r}0.177 \\
8 \\
\end{array}$ & $\begin{array}{r}1 . \\
5\end{array}$ & $\begin{array}{r}0.3 \\
6 \\
\end{array}$ & $\begin{array}{r}1054 . \\
7\end{array}$ & 14.9 & 1079.9 & 28.0 & $\begin{array}{r}1130 . \\
9 \\
\end{array}$ & 78.2 & 1130.9 & 78.2 & 93.3 \\
\hline $\begin{array}{l}\text { BU0755- } \\
92\end{array}$ & 331 & 40230 & 3.5 & $\begin{array}{r}12.79 \\
37\end{array}$ & 0.8 & 1.8206 & 2.5 & $\begin{array}{r}0.168 \\
9\end{array}$ & $\begin{array}{r}2 . \\
4\end{array}$ & $\begin{array}{r}0.9 \\
5\end{array}$ & $\begin{array}{r}1006 . \\
2\end{array}$ & 22.5 & 1052.9 & 16.7 & $\begin{array}{r}1151 . \\
0\end{array}$ & 15.9 & 1151.0 & 15.9 & 87.4 \\
\hline $\begin{array}{l}\text { BU0755- } \\
98\end{array}$ & 150 & 14025 & 1.1 & $\begin{array}{r}12.79 \\
21\end{array}$ & 2.4 & 1.9047 & 2.9 & $\begin{array}{r}0.176 \\
7\end{array}$ & $\begin{array}{r}1 . \\
7\end{array}$ & $\begin{array}{r}0.5 \\
8\end{array}$ & $\begin{array}{r}1049 . \\
0\end{array}$ & 16.1 & 1082.7 & 19.2 & $\begin{array}{r}1151 . \\
3\end{array}$ & 46.7 & 1151.3 & 46.7 & 91.1 \\
\hline $\begin{array}{l}\text { BU0755- } \\
16\end{array}$ & 646 & 70866 & 2.8 & $\begin{array}{r}12.70 \\
32\end{array}$ & 2.5 & 2.2255 & 3.0 & $\begin{array}{r}0.205 \\
0\end{array}$ & $\begin{array}{l}1 . \\
5\end{array}$ & $\begin{array}{r}0.5 \\
1\end{array}$ & $\begin{array}{r}1202 . \\
3\end{array}$ & 16.7 & 1189.1 & 20.7 & $\begin{array}{r}1165 . \\
1\end{array}$ & 50.4 & 1165.1 & 50.4 & $\begin{array}{r}103 . \\
2\end{array}$ \\
\hline $\begin{array}{l}\text { BU0755- } \\
25\end{array}$ & 418 & 42225 & 4.0 & $\begin{array}{r}12.64 \\
18 \\
\end{array}$ & 1.0 & 2.1871 & 1.6 & $\begin{array}{r}0.200 \\
5 \\
\end{array}$ & $\begin{array}{r}1 . \\
2\end{array}$ & $\begin{array}{r}0.7 \\
6 \\
\end{array}$ & $\begin{array}{r}1178 . \\
2\end{array}$ & 13.1 & 1176.9 & 11.2 & $\begin{array}{r}1174 . \\
7\end{array}$ & 20.6 & 1174.7 & 20.6 & $\begin{array}{r}100 . \\
3\end{array}$ \\
\hline BU0755-1 & 412 & 37614 & 18.8 & $\begin{array}{r}12.60 \\
92\end{array}$ & 1.9 & 2.2136 & 2.4 & $\begin{array}{r}0.202 \\
4\end{array}$ & $\begin{array}{r}1 . \\
5\end{array}$ & $\begin{array}{r}0.6 \\
3\end{array}$ & $\begin{array}{r}1188 . \\
4\end{array}$ & 16.2 & 1185.3 & 16.6 & $\begin{array}{r}1179 . \\
8\end{array}$ & 36.6 & 1179.8 & 36.6 & $\begin{array}{r}100 . \\
7\end{array}$ \\
\hline $\begin{array}{l}\text { BU0755- } \\
55\end{array}$ & 258 & 20424 & $\begin{array}{r}191 . \\
8\end{array}$ & $\begin{array}{r}12.59 \\
98\end{array}$ & 1.9 & 2.1085 & 2.5 & $\begin{array}{r}0.192 \\
7\end{array}$ & $\begin{array}{r}1 . \\
7\end{array}$ & $\begin{array}{r}0.6 \\
6\end{array}$ & $\begin{array}{r}1135 . \\
9\end{array}$ & 17.2 & 1151.6 & 17.1 & $\begin{array}{r}1181 . \\
3\end{array}$ & 36.8 & 1181.3 & 36.8 & 96.2 \\
\hline $\begin{array}{l}\text { BU0755- } \\
85\end{array}$ & 407 & 21027 & 0.9 & $\begin{array}{r}12.41 \\
89\end{array}$ & 1.8 & 1.9355 & 2.8 & $\begin{array}{r}0.174 \\
3\end{array}$ & $\begin{array}{r}2 . \\
1\end{array}$ & $\begin{array}{r}0.7 \\
6\end{array}$ & $\begin{array}{r}1035 . \\
9\end{array}$ & 20.4 & 1093.4 & 18.8 & $\begin{array}{r}1209 . \\
8\end{array}$ & 36.1 & 1209.8 & 36.1 & 85.6 \\
\hline $\begin{array}{l}\text { BU0755- } \\
91\end{array}$ & $\begin{array}{r}103 \\
9 \\
\end{array}$ & $\begin{array}{r}10991 \\
7 \\
\end{array}$ & 4.5 & $\begin{array}{r}12.30 \\
32 \\
\end{array}$ & 1.5 & 2.0077 & 4.2 & $\begin{array}{r}0.179 \\
1 \\
\end{array}$ & $\begin{array}{r}3 . \\
9 \\
\end{array}$ & $\begin{array}{r}0.9 \\
4 \\
\end{array}$ & $\begin{array}{r}1062 . \\
3 \\
\end{array}$ & 38.6 & 1118.1 & 28.6 & $\begin{array}{r}1228 . \\
2 \\
\end{array}$ & 29.3 & 1228.2 & 29.3 & 86.5 \\
\hline $\begin{array}{l}\text { BU0755- } \\
97\end{array}$ & 360 & 26100 & 1.7 & $\begin{array}{r}12.15 \\
90\end{array}$ & 1.2 & 2.0507 & 2.1 & $\begin{array}{r}0.180 \\
8\end{array}$ & $\begin{array}{r}1 . \\
8\end{array}$ & $\begin{array}{r}0.8 \\
3\end{array}$ & $\begin{array}{r}1071 . \\
5\end{array}$ & 17.4 & 1132.5 & 14.5 & $\begin{array}{r}1251 . \\
3\end{array}$ & 23.3 & 1251.3 & 23.3 & 85.6 \\
\hline BU0755-7 & 665 & 32835 & 4.9 & $\begin{array}{r}12.13 \\
36\end{array}$ & 1.9 & 1.9834 & 2.5 & $\begin{array}{r}0.174 \\
5\end{array}$ & $\begin{array}{l}1 . \\
7\end{array}$ & $\begin{array}{r}0.6 \\
6\end{array}$ & $\begin{array}{r}1037 . \\
1\end{array}$ & 15.9 & 1109.9 & 16.9 & $\begin{array}{r}1255 . \\
4\end{array}$ & 36.6 & 1255.4 & 36.6 & 82.6 \\
\hline
\end{tabular}




\begin{tabular}{|c|c|c|c|c|c|c|c|c|c|c|c|c|c|c|c|c|c|c|c|}
\hline $\begin{array}{l}\text { BU0755- } \\
67\end{array}$ & 265 & 24114 & 2.1 & $\begin{array}{r}11.98 \\
28 \\
\end{array}$ & 2.6 & 2.0011 & 6.4 & $\begin{array}{r}0.173 \\
9 \\
\end{array}$ & $\begin{array}{r}5 . \\
9 \\
\end{array}$ & $\begin{array}{r}0.9 \\
2 \\
\end{array}$ & $\begin{array}{r}1033 . \\
6 \\
\end{array}$ & 56.4 & 1115.9 & 43.6 & $\begin{array}{r}1279 . \\
8 \\
\end{array}$ & 49.9 & 1279.8 & 49.9 & 80.8 \\
\hline $\begin{array}{l}\text { BU0755- } \\
63\end{array}$ & 373 & 40155 & 1.5 & $\begin{array}{r}11.94 \\
98\end{array}$ & 2.5 & 2.3391 & 4.6 & $\begin{array}{r}0.202 \\
7\end{array}$ & $\begin{array}{r}3 . \\
8\end{array}$ & $\begin{array}{r}0.8 \\
4\end{array}$ & $\begin{array}{r}1189 . \\
9\end{array}$ & 41.6 & 1224.2 & 32.5 & $\begin{array}{r}1285 . \\
2\end{array}$ & 48.5 & 1285.2 & 48.5 & 92.6 \\
\hline $\begin{array}{l}\text { BU0755- } \\
103\end{array}$ & 292 & 31890 & 1.7 & $\begin{array}{r}11.83 \\
05\end{array}$ & 1.4 & 2.3589 & 2.6 & $\begin{array}{r}0.202 \\
4\end{array}$ & $\begin{array}{r}2 . \\
2\end{array}$ & $\begin{array}{r}0.8 \\
4\end{array}$ & $\begin{array}{r}1188 . \\
2\end{array}$ & 23.8 & 1230.3 & 18.6 & $\begin{array}{r}1304 . \\
7\end{array}$ & 27.6 & 1304.7 & 27.6 & 91.1 \\
\hline $\begin{array}{l}\text { BU0755- } \\
23\end{array}$ & $\begin{array}{r}157 \\
2 \\
\end{array}$ & 68043 & 2.5 & $\begin{array}{r}11.80 \\
29 \\
\end{array}$ & 2.4 & 2.2423 & 4.6 & $\begin{array}{r}0.191 \\
9 \\
\end{array}$ & $\begin{array}{r}4 . \\
0\end{array}$ & $\begin{array}{r}0.8 \\
6 \\
\end{array}$ & $\begin{array}{r}1131 . \\
9\end{array}$ & 41.5 & 1194.4 & 32.6 & $\begin{array}{r}1309 . \\
2\end{array}$ & 45.6 & 1309.2 & 45.6 & 86.5 \\
\hline $\begin{array}{l}\text { BU0755- } \\
68\end{array}$ & 191 & 23424 & 2.6 & $\begin{array}{r}11.60 \\
13 \\
\end{array}$ & 1.4 & 2.1439 & 3.5 & $\begin{array}{r}0.180 \\
4 \\
\end{array}$ & $\begin{array}{r}3 . \\
2 \\
\end{array}$ & $\begin{array}{r}0.9 \\
2 \\
\end{array}$ & $\begin{array}{r}1069 . \\
1 \\
\end{array}$ & 31.7 & 1163.1 & 24.2 & $\begin{array}{r}1342 . \\
6 \\
\end{array}$ & 26.1 & 1342.6 & 26.1 & 79.6 \\
\hline $\begin{array}{l}\text { BU0755- } \\
59\end{array}$ & 887 & 75828 & 10.0 & $\begin{array}{r}11.45 \\
46 \\
\end{array}$ & 1.4 & 2.4205 & 2.7 & $\begin{array}{r}0.201 \\
1 \\
\end{array}$ & $\begin{array}{r}2 . \\
4\end{array}$ & $\begin{array}{r}0.8 \\
7 \\
\end{array}$ & $\begin{array}{r}1181 . \\
1\end{array}$ & 25.8 & 1248.7 & 19.8 & $\begin{array}{r}1367 . \\
1\end{array}$ & 26.2 & 1367.1 & 26.2 & 86.4 \\
\hline $\begin{array}{l}\text { BU0755- } \\
57\end{array}$ & 248 & 49236 & 2.0 & $\begin{array}{r}11.40 \\
88\end{array}$ & 2.1 & 2.6175 & 4.1 & $\begin{array}{r}0.216 \\
6\end{array}$ & $\begin{array}{r}3 . \\
5\end{array}$ & $\begin{array}{r}0.8 \\
5\end{array}$ & $\begin{array}{r}1263 . \\
8\end{array}$ & 39.9 & 1305.6 & 29.9 & $\begin{array}{r}1374 . \\
8\end{array}$ & 40.8 & 1374.8 & 40.8 & 91.9 \\
\hline $\begin{array}{l}\text { BU0755- } \\
100\end{array}$ & 271 & 35274 & 2.6 & $\begin{array}{r}11.37 \\
08 \\
\end{array}$ & 1.9 & 2.5854 & 2.9 & $\begin{array}{r}0.213 \\
2 \\
\end{array}$ & $\begin{array}{r}2 . \\
2 \\
\end{array}$ & $\begin{array}{r}0.7 \\
6 \\
\end{array}$ & $\begin{array}{r}1245 . \\
9 \\
\end{array}$ & 25.3 & 1296.5 & 21.5 & $\begin{array}{r}1381 . \\
3 \\
\end{array}$ & 36.7 & 1381.3 & 36.7 & 90.2 \\
\hline $\begin{array}{l}\text { BU0755- } \\
62\end{array}$ & 959 & $\begin{array}{r}12768 \\
0\end{array}$ & 1.9 & $\begin{array}{r}11.31 \\
91\end{array}$ & 2.5 & 2.6068 & 3.8 & $\begin{array}{r}0.214 \\
0\end{array}$ & $\begin{array}{r}2 . \\
8\end{array}$ & $\begin{array}{r}0.7 \\
5\end{array}$ & $\begin{array}{r}1250 . \\
1\end{array}$ & 32.3 & 1302.6 & 27.8 & $\begin{array}{r}1390 . \\
0\end{array}$ & 48.2 & 1390.0 & 48.2 & 89.9 \\
\hline $\begin{array}{l}\text { BU0755- } \\
19\end{array}$ & 564 & 52974 & 5.0 & $\begin{array}{r}11.31 \\
87 \\
\end{array}$ & 2.8 & 2.8881 & 3.1 & $\begin{array}{r}0.237 \\
1 \\
\end{array}$ & $\begin{array}{r}1 . \\
4\end{array}$ & $\begin{array}{r}0.4 \\
5 \\
\end{array}$ & $\begin{array}{r}1371 . \\
5 \\
\end{array}$ & 17.5 & 1378.8 & 23.6 & $\begin{array}{r}1390 . \\
1 \\
\end{array}$ & 53.4 & 1390.1 & 53.4 & 98.7 \\
\hline BU0755-8 & 146 & 15993 & 1.8 & $\begin{array}{r}11.29 \\
77\end{array}$ & 1.9 & 2.9231 & 2.1 & $\begin{array}{r}0.239 \\
5\end{array}$ & $\begin{array}{r}0 . \\
9\end{array}$ & $\begin{array}{r}0.4 \\
4\end{array}$ & $\begin{array}{r}1384 . \\
2\end{array}$ & 11.5 & 1387.9 & 15.9 & $\begin{array}{r}1393 . \\
6\end{array}$ & 36.3 & 1393.6 & 36.3 & 99.3 \\
\hline $\begin{array}{l}\text { BU0755- } \\
44\end{array}$ & 511 & 87723 & 8.3 & $\begin{array}{r}11.29 \\
10\end{array}$ & 1.1 & 2.8442 & 4.8 & $\begin{array}{r}0.232 \\
9\end{array}$ & $\begin{array}{r}4 . \\
7\end{array}$ & $\begin{array}{r}0.9 \\
7\end{array}$ & $\begin{array}{r}1349 . \\
7\end{array}$ & 56.8 & 1367.3 & 36.0 & $\begin{array}{r}1394 . \\
8\end{array}$ & 20.7 & 1394.8 & 20.7 & 96.8 \\
\hline $\begin{array}{l}\text { BU0755- } \\
95\end{array}$ & 615 & 73974 & 3.1 & $\begin{array}{r}11.20 \\
58 \\
\end{array}$ & 1.0 & 2.7853 & 2.9 & $\begin{array}{r}0.226 \\
4 \\
\end{array}$ & $\begin{array}{r}2 . \\
7\end{array}$ & $\begin{array}{r}0.9 \\
4 \\
\end{array}$ & $\begin{array}{r}1315 . \\
4\end{array}$ & 32.6 & 1351.6 & 21.8 & $\begin{array}{r}1409 . \\
3\end{array}$ & 19.0 & 1409.3 & 19.0 & 93.3 \\
\hline $\begin{array}{l}\text { BU0755- } \\
47\end{array}$ & 277 & 46713 & 1.9 & $\begin{array}{r}11.10 \\
89 \\
\end{array}$ & 1.7 & 2.9717 & 3.7 & $\begin{array}{r}0.239 \\
4 \\
\end{array}$ & $\begin{array}{r}3 . \\
3 \\
\end{array}$ & $\begin{array}{r}0.8 \\
8 \\
\end{array}$ & $\begin{array}{r}1383 . \\
7 \\
\end{array}$ & 40.7 & 1400.4 & 28.1 & $\begin{array}{r}1425 . \\
9 \\
\end{array}$ & 32.9 & 1425.9 & 32.9 & 97.0 \\
\hline BU0755-6 & 462 & 52821 & 1.6 & $\begin{array}{r}11.10 \\
24 \\
\end{array}$ & 1.5 & 3.0679 & 1.9 & $\begin{array}{r}0.247 \\
0 \\
\end{array}$ & $\begin{array}{r}1 . \\
3\end{array}$ & $\begin{array}{r}0.6 \\
5 \\
\end{array}$ & $\begin{array}{r}1423 . \\
1 \\
\end{array}$ & 16.0 & 1424.7 & 14.7 & $\begin{array}{r}1427 . \\
0 \\
\end{array}$ & 27.7 & 1427.0 & 27.7 & 99.7 \\
\hline $\begin{array}{l}\text { BU0755- } \\
29\end{array}$ & 655 & 71892 & 8.2 & $\begin{array}{r}11.05 \\
30\end{array}$ & 0.9 & 3.0228 & 2.0 & $\begin{array}{r}0.242 \\
3\end{array}$ & $\begin{array}{l}1 . \\
7\end{array}$ & $\begin{array}{r}0.8 \\
8\end{array}$ & $\begin{array}{r}1398 . \\
7\end{array}$ & 21.6 & 1413.4 & 14.9 & $\begin{array}{r}1435 . \\
5\end{array}$ & 17.7 & 1435.5 & 17.7 & 97.4 \\
\hline $\begin{array}{l}\text { BU0755- } \\
87\end{array}$ & $\begin{array}{r}106 \\
0 \\
\end{array}$ & $\begin{array}{r}11997 \\
0 \\
\end{array}$ & 1.5 & $\begin{array}{r}10.93 \\
07 \\
\end{array}$ & 1.2 & 2.5127 & 3.5 & $\begin{array}{r}0.199 \\
2 \\
\end{array}$ & $\begin{array}{r}3 . \\
3 \\
\end{array}$ & $\begin{array}{r}0.9 \\
4 \\
\end{array}$ & $\begin{array}{r}1171 . \\
0 \\
\end{array}$ & 34.8 & 1275.7 & 25.2 & $\begin{array}{r}1456 . \\
7 \\
\end{array}$ & 23.2 & 1456.7 & 23.2 & 80.4 \\
\hline $\begin{array}{l}\text { BU0755- } \\
71\end{array}$ & 497 & 61350 & 1.9 & $\begin{array}{r}10.79 \\
06 \\
\end{array}$ & 3.0 & 2.6349 & 3.3 & $\begin{array}{r}0.206 \\
2 \\
\end{array}$ & $\begin{array}{r}1 . \\
5\end{array}$ & $\begin{array}{r}0.4 \\
6 \\
\end{array}$ & $\begin{array}{r}1208 . \\
6 \\
\end{array}$ & 16.6 & 1310.4 & 24.4 & $\begin{array}{r}1481 . \\
2 \\
\end{array}$ & 55.9 & 1481.2 & 55.9 & 81.6 \\
\hline $\begin{array}{l}\text { BU0755- } \\
43\end{array}$ & $\begin{array}{r}121 \\
3 \\
\end{array}$ & $\begin{array}{r}14202 \\
6 \\
\end{array}$ & 2.6 & $\begin{array}{r}10.55 \\
37 \\
\end{array}$ & 1.7 & 2.9972 & 5.0 & $\begin{array}{r}0.229 \\
4 \\
\end{array}$ & $\begin{array}{r}4 . \\
7 \\
\end{array}$ & $\begin{array}{r}0.9 \\
4\end{array}$ & $\begin{array}{r}1331 . \\
4\end{array}$ & 57.0 & 1406.9 & 38.3 & $\begin{array}{r}1523 . \\
2\end{array}$ & 31.5 & 1523.2 & 31.5 & 87.4 \\
\hline $\begin{array}{l}\text { BU0755- } \\
39\end{array}$ & $\begin{array}{r}150 \\
9\end{array}$ & $\begin{array}{r}12364 \\
5\end{array}$ & 1.5 & $\begin{array}{r}10.45 \\
76\end{array}$ & 1.5 & 3.4740 & 2.1 & $\begin{array}{r}0.263 \\
5\end{array}$ & $\begin{array}{r}1 . \\
5\end{array}$ & $\begin{array}{r}0.7 \\
0\end{array}$ & $\begin{array}{r}1507 . \\
6\end{array}$ & 19.6 & 1521.3 & 16.3 & $\begin{array}{r}1540 . \\
4\end{array}$ & 27.6 & 1540.4 & 27.6 & 97.9 \\
\hline $\begin{array}{l}\text { BU0755- } \\
41\end{array}$ & 371 & 37356 & 1.4 & $\begin{array}{r}10.45 \\
05\end{array}$ & 2.5 & 2.8950 & 5.9 & $\begin{array}{r}0.219 \\
4\end{array}$ & $\begin{array}{r}5 . \\
3\end{array}$ & $\begin{array}{r}0.9 \\
0\end{array}$ & $\begin{array}{r}1278 . \\
8\end{array}$ & 61.7 & 1380.6 & 44.5 & $\begin{array}{r}1541 . \\
7\end{array}$ & 47.8 & 1541.7 & 47.8 & 83.0 \\
\hline $\begin{array}{l}\text { BU0755- } \\
84\end{array}$ & 256 & 28323 & 1.6 & $\begin{array}{r}10.37 \\
96\end{array}$ & 1.1 & 3.1400 & 1.9 & $\begin{array}{r}0.236 \\
4\end{array}$ & $\begin{array}{r}1 . \\
6\end{array}$ & $\begin{array}{r}0.8 \\
1\end{array}$ & $\begin{array}{r}1367 . \\
8\end{array}$ & 19.5 & 1442.6 & 15.0 & $\begin{array}{r}1554 . \\
5\end{array}$ & 21.4 & 1554.5 & 21.4 & 88.0 \\
\hline $\begin{array}{l}\text { BU0755- } \\
83\end{array}$ & 157 & 25803 & 1.9 & $\begin{array}{r}10.09 \\
76 \\
\end{array}$ & 1.4 & 3.5044 & 1.7 & $\begin{array}{r}0.256 \\
6 \\
\end{array}$ & $\begin{array}{r}1 . \\
0\end{array}$ & $\begin{array}{r}0.5 \\
8 \\
\end{array}$ & $\begin{array}{r}1472 . \\
7 \\
\end{array}$ & 13.4 & 1528.2 & 13.8 & $\begin{array}{r}1606 . \\
0 \\
\end{array}$ & 26.5 & 1606.0 & 26.5 & 91.7 \\
\hline $\begin{array}{l}\text { BU0755- } \\
18\end{array}$ & 269 & 55611 & 1.6 & $\begin{array}{r}10.08 \\
40 \\
\end{array}$ & 1.4 & 3.9229 & 3.8 & $\begin{array}{r}0.286 \\
9 \\
\end{array}$ & $\begin{array}{r}3 . \\
5 \\
\end{array}$ & $\begin{array}{r}0.9 \\
3 \\
\end{array}$ & $\begin{array}{r}1626 . \\
1 \\
\end{array}$ & 50.9 & 1618.4 & 30.8 & $\begin{array}{r}1608 . \\
5 \\
\end{array}$ & 25.9 & 1608.5 & 25.9 & $\begin{array}{r}101 . \\
1 \\
\end{array}$ \\
\hline BU0755- & 123 & 13653 & 1.0 & 10.04 & 2.7 & 3.7548 & 3.0 & 0.273 & 1. & 0.4 & 1558. & 19.8 & 1583.1 & 24.2 & 1616. & 49.4 & 1616.0 & 49.4 & 96.4 \\
\hline
\end{tabular}




\begin{tabular}{|c|c|c|c|c|c|c|c|c|c|c|c|c|c|c|c|c|c|c|c|}
\hline 35 & & & & 35 & & & & 5 & 4 & 7 & 6 & & & & 0 & & & & \\
\hline $\begin{array}{l}\text { BU0755- } \\
42\end{array}$ & $\begin{array}{r}132 \\
3\end{array}$ & 70467 & 4.6 & $\begin{array}{r}10.00 \\
52\end{array}$ & 1.5 & 3.4241 & 1.8 & $\begin{array}{r}0.248 \\
5\end{array}$ & $\begin{array}{r}0 . \\
9\end{array}$ & $\begin{array}{r}0.5 \\
2\end{array}$ & $\begin{array}{r}1430 . \\
6\end{array}$ & 11.9 & 1509.9 & 13.9 & $\begin{array}{r}1623 . \\
1\end{array}$ & 28.1 & 1623.1 & 28.1 & 88.1 \\
\hline $\begin{array}{l}\text { BU0755- } \\
82\end{array}$ & $\begin{array}{r}307 \\
8\end{array}$ & $\begin{array}{r}13659 \\
0\end{array}$ & 1.8 & $\begin{array}{r}9.854 \\
1\end{array}$ & 1.7 & 2.9100 & 2.4 & $\begin{array}{r}0.208 \\
0\end{array}$ & $\begin{array}{l}1 . \\
7\end{array}$ & $\begin{array}{r}0.7 \\
2\end{array}$ & $\begin{array}{r}1218 . \\
0\end{array}$ & 19.2 & 1384.5 & 18.2 & $\begin{array}{r}1651 . \\
4\end{array}$ & 31.0 & 1651.4 & 31.0 & 73.8 \\
\hline BU0755-9 & 501 & 80769 & 1.9 & $\begin{array}{r}9.851 \\
2\end{array}$ & 1.7 & 4.1124 & 1.9 & $\begin{array}{r}0.293 \\
8\end{array}$ & $\begin{array}{r}0 . \\
8\end{array}$ & $\begin{array}{r}0.4 \\
2\end{array}$ & $\begin{array}{r}1660 . \\
6\end{array}$ & 11.9 & 1656.8 & 15.7 & $\begin{array}{r}1651 . \\
9\end{array}$ & 32.3 & 1651.9 & 32.3 & $\begin{array}{r}100 . \\
5\end{array}$ \\
\hline $\begin{array}{l}\text { BU0755- } \\
49\end{array}$ & 591 & 35034 & 1.6 & $\begin{array}{r}9.841 \\
2 \\
\end{array}$ & 2.4 & 2.9954 & 4.3 & $\begin{array}{r}0.213 \\
8 \\
\end{array}$ & $\begin{array}{r}3 . \\
6 \\
\end{array}$ & $\begin{array}{r}0.8 \\
4\end{array}$ & $\begin{array}{r}1249 . \\
0\end{array}$ & 41.1 & 1406.5 & 32.9 & $\begin{array}{r}1653 . \\
8\end{array}$ & 43.6 & 1653.8 & 43.6 & 75.5 \\
\hline $\begin{array}{l}\text { BU0755- } \\
40\end{array}$ & $\begin{array}{r}166 \\
5\end{array}$ & 85818 & 1.5 & $\begin{array}{r}9.831 \\
3\end{array}$ & 2.5 & 3.1266 & 3.9 & $\begin{array}{r}0.222 \\
9\end{array}$ & $\begin{array}{r}3 . \\
0\end{array}$ & $\begin{array}{r}0.7 \\
6\end{array}$ & $\begin{array}{r}1297 . \\
4\end{array}$ & 34.9 & 1439.2 & 30.0 & $\begin{array}{r}1655 . \\
7\end{array}$ & 46.7 & 1655.7 & 46.7 & 78.4 \\
\hline $\begin{array}{l}\text { BU0755- } \\
88\end{array}$ & $\begin{array}{r}190 \\
3\end{array}$ & $\begin{array}{r}12681 \\
9\end{array}$ & 4.3 & $\begin{array}{r}9.813 \\
3\end{array}$ & 1.1 & 3.4536 & 3.4 & $\begin{array}{r}0.245 \\
8\end{array}$ & $\begin{array}{r}3 . \\
2\end{array}$ & $\begin{array}{r}0.9 \\
4 \\
\end{array}$ & $\begin{array}{r}1416 . \\
8 \\
\end{array}$ & 40.7 & 1516.7 & 26.7 & $\begin{array}{r}1659 . \\
1 \\
\end{array}$ & 20.6 & 1659.1 & 20.6 & 85.4 \\
\hline $\begin{array}{l}\text { BU0755- } \\
102\end{array}$ & 339 & 54513 & 2.4 & $\begin{array}{r}9.810 \\
7\end{array}$ & 1.5 & 3.6930 & 1.9 & $\begin{array}{r}0.262 \\
8\end{array}$ & $\begin{array}{r}1 . \\
2\end{array}$ & $\begin{array}{r}0.6 \\
3\end{array}$ & $\begin{array}{r}1504 . \\
0\end{array}$ & 16.1 & 1569.9 & 15.2 & $\begin{array}{r}1659 . \\
5\end{array}$ & 27.2 & 1659.5 & 27.2 & 90.6 \\
\hline $\begin{array}{l}\text { BU0755- } \\
77\end{array}$ & 191 & 34566 & 2.4 & $\begin{array}{r}9.809 \\
9 \\
\end{array}$ & 2.7 & 3.5246 & 3.5 & $\begin{array}{r}0.250 \\
8 \\
\end{array}$ & $\begin{array}{r}2 . \\
1\end{array}$ & $\begin{array}{r}0.6 \\
2 \\
\end{array}$ & $\begin{array}{r}1442 . \\
4\end{array}$ & 27.7 & 1532.7 & 27.4 & $\begin{array}{r}1659 . \\
7\end{array}$ & 50.6 & 1659.7 & 50.6 & 86.9 \\
\hline $\begin{array}{l}\text { BU0755- } \\
104\end{array}$ & 138 & 26352 & 1.9 & $\begin{array}{r}9.796 \\
0 \\
\end{array}$ & 2.5 & 3.7358 & 2.5 & $\begin{array}{r}0.265 \\
4 \\
\end{array}$ & $\begin{array}{r}0 . \\
6 \\
\end{array}$ & $\begin{array}{r}0.2 \\
4 \\
\end{array}$ & $\begin{array}{r}1517 . \\
5 \\
\end{array}$ & 8.1 & 1579.1 & 20.3 & $\begin{array}{r}1662 . \\
3 \\
\end{array}$ & 45.6 & 1662.3 & 45.6 & 91.3 \\
\hline $\begin{array}{l}\text { BU0755- } \\
22\end{array}$ & $\begin{array}{r}175 \\
2 \\
\end{array}$ & 68841 & 5.3 & $\begin{array}{r}9.703 \\
9 \\
\end{array}$ & 1.9 & 3.5231 & 2.6 & $\begin{array}{r}0.248 \\
0 \\
\end{array}$ & $\begin{array}{r}1 . \\
8\end{array}$ & $\begin{array}{r}0.6 \\
8 \\
\end{array}$ & $\begin{array}{r}1427 . \\
9\end{array}$ & 23.2 & 1532.4 & 20.9 & $\begin{array}{r}1679 . \\
8\end{array}$ & 35.7 & 1679.8 & 35.7 & 85.0 \\
\hline $\begin{array}{l}\text { BU0755- } \\
75\end{array}$ & $\begin{array}{r}219 \\
3\end{array}$ & $\begin{array}{r}16349 \\
1\end{array}$ & 2.0 & $\begin{array}{r}9.702 \\
5\end{array}$ & 2.2 & 3.1948 & 3.2 & $\begin{array}{r}0.224 \\
8\end{array}$ & $\begin{array}{r}2 . \\
3\end{array}$ & $\begin{array}{r}0.7 \\
3\end{array}$ & $\begin{array}{r}1307 . \\
3\end{array}$ & 27.5 & 1455.9 & 24.5 & $\begin{array}{r}1680 . \\
0\end{array}$ & 39.7 & 1680.0 & 39.7 & 77.8 \\
\hline $\begin{array}{l}\text { BU0755- } \\
80\end{array}$ & $\begin{array}{r}124 \\
7\end{array}$ & $\begin{array}{r}14144 \\
1\end{array}$ & 3.1 & $\begin{array}{r}9.668 \\
5\end{array}$ & 1.1 & 3.5355 & 2.7 & $\begin{array}{r}0.247 \\
9\end{array}$ & $\begin{array}{r}2 . \\
4\end{array}$ & $\begin{array}{r}0.9 \\
2\end{array}$ & $\begin{array}{r}1427 . \\
7\end{array}$ & 31.1 & 1535.2 & 21.0 & $\begin{array}{r}1686 . \\
5\end{array}$ & 19.6 & 1686.5 & 19.6 & 84.7 \\
\hline $\begin{array}{l}\text { BU0755- } \\
58\end{array}$ & 965 & 30672 & 0.9 & $\begin{array}{r}9.665 \\
5\end{array}$ & 3.2 & 3.3841 & 4.6 & $\begin{array}{r}0.237 \\
2\end{array}$ & $\begin{array}{r}3 . \\
3\end{array}$ & $\begin{array}{r}0.7 \\
3\end{array}$ & $\begin{array}{r}1372 . \\
3\end{array}$ & 41.2 & 1500.7 & 35.9 & $\begin{array}{r}1687 . \\
1\end{array}$ & 58.1 & 1687.1 & 58.1 & 81.3 \\
\hline $\begin{array}{l}\text { BU0755- } \\
34\end{array}$ & 502 & 62634 & 2.0 & $\begin{array}{r}9.643 \\
3 \\
\end{array}$ & 2.1 & 4.0890 & 2.9 & $\begin{array}{r}0.286 \\
0 \\
\end{array}$ & $\begin{array}{r}2 . \\
0\end{array}$ & $\begin{array}{r}0.6 \\
8 \\
\end{array}$ & $\begin{array}{r}1621 . \\
4\end{array}$ & 28.0 & 1652.1 & 23.3 & $\begin{array}{r}1691 . \\
3 \\
\end{array}$ & 38.5 & 1691.3 & 38.5 & 95.9 \\
\hline BU0755-4 & 734 & $\begin{array}{r}11094 \\
6\end{array}$ & 2.3 & $\begin{array}{r}9.503 \\
1\end{array}$ & 1.2 & 4.4172 & 2.5 & $\begin{array}{r}0.304 \\
4\end{array}$ & $\begin{array}{r}2 . \\
2\end{array}$ & $\begin{array}{r}0.8 \\
8\end{array}$ & $\begin{array}{r}1713 . \\
3\end{array}$ & 32.6 & 1715.6 & 20.5 & $\begin{array}{r}1718 . \\
3\end{array}$ & 22.1 & 1718.3 & 22.1 & 99.7 \\
\hline $\begin{array}{l}\text { BU0755- } \\
24\end{array}$ & 581 & 65424 & 1.1 & $\begin{array}{r}9.478 \\
3\end{array}$ & 4.5 & 4.3056 & 5.2 & $\begin{array}{r}0.296 \\
0\end{array}$ & $\begin{array}{r}2 . \\
7\end{array}$ & $\begin{array}{r}0.5 \\
2\end{array}$ & $\begin{array}{r}1671 . \\
3\end{array}$ & 39.8 & 1694.4 & 43.1 & $\begin{array}{r}1723 . \\
1\end{array}$ & 82.3 & 1723.1 & 82.3 & 97.0 \\
\hline $\begin{array}{l}\text { BU0755- } \\
79\end{array}$ & 131 & 22347 & 1.8 & $\begin{array}{r}9.420 \\
5\end{array}$ & 1.6 & 4.2441 & 2.2 & $\begin{array}{r}0.290 \\
0\end{array}$ & $\begin{array}{l}1 . \\
5\end{array}$ & $\begin{array}{r}0.6 \\
8\end{array}$ & $\begin{array}{r}1641 . \\
4\end{array}$ & 21.9 & 1682.6 & 18.2 & $\begin{array}{r}1734 . \\
3\end{array}$ & 29.6 & 1734.3 & 29.6 & 94.6 \\
\hline $\begin{array}{l}\text { BU0755- } \\
26\end{array}$ & 436 & 70902 & 1.3 & $\begin{array}{r}9.413 \\
3\end{array}$ & 0.9 & 4.5142 & 1.5 & $\begin{array}{r}0.308 \\
2\end{array}$ & $\begin{array}{r}1 . \\
2\end{array}$ & $\begin{array}{r}0.7 \\
8\end{array}$ & $\begin{array}{r}1731 . \\
8\end{array}$ & 17.6 & 1733.6 & 12.4 & $\begin{array}{r}1735 . \\
7\end{array}$ & 17.1 & 1735.7 & 17.1 & 99.8 \\
\hline $\begin{array}{l}\text { BU0755- } \\
31\end{array}$ & 252 & 29676 & 1.5 & $\begin{array}{r}9.390 \\
6 \\
\end{array}$ & 2.0 & 4.3500 & 2.3 & $\begin{array}{r}0.296 \\
3 \\
\end{array}$ & $\begin{array}{r}1 . \\
1\end{array}$ & $\begin{array}{r}0.4 \\
9 \\
\end{array}$ & $\begin{array}{r}1672 . \\
8\end{array}$ & 16.5 & 1702.9 & 18.9 & $\begin{array}{r}1740 . \\
2 \\
\end{array}$ & 36.5 & 1740.2 & 36.5 & 96.1 \\
\hline $\begin{array}{l}\text { BU0755- } \\
54\end{array}$ & 800 & 81492 & 7.3 & $\begin{array}{r}9.366 \\
0\end{array}$ & 2.2 & 4.3823 & 2.3 & $\begin{array}{r}0.297 \\
7\end{array}$ & $\begin{array}{r}0 . \\
8\end{array}$ & $\begin{array}{r}0.3 \\
5\end{array}$ & $\begin{array}{r}1679 . \\
8\end{array}$ & 12.1 & 1709.0 & 19.3 & $\begin{array}{r}1745 . \\
0\end{array}$ & 40.1 & 1745.0 & 40.1 & 96.3 \\
\hline $\begin{array}{l}\text { BU0755- } \\
96\end{array}$ & 296 & 41841 & 1.8 & $\begin{array}{r}9.361 \\
3\end{array}$ & 0.7 & 3.9454 & 1.4 & $\begin{array}{r}0.267 \\
9\end{array}$ & $\begin{array}{r}1 . \\
2\end{array}$ & $\begin{array}{r}0.8 \\
6\end{array}$ & $\begin{array}{r}1530 . \\
0\end{array}$ & 16.5 & 1623.0 & 11.4 & $\begin{array}{r}1745 . \\
9\end{array}$ & 13.4 & 1745.9 & 13.4 & 87.6 \\
\hline $\begin{array}{l}\text { BU0755- } \\
64\end{array}$ & 333 & 43131 & 1.4 & $\begin{array}{r}9.354 \\
1\end{array}$ & 1.1 & 4.3017 & 2.3 & $\begin{array}{r}0.291 \\
8\end{array}$ & $\begin{array}{l}2 . \\
0\end{array}$ & $\begin{array}{r}0.8 \\
8\end{array}$ & $\begin{array}{r}1650 . \\
7\end{array}$ & 29.4 & 1693.7 & 18.9 & $\begin{array}{r}1747 . \\
3\end{array}$ & 19.8 & 1747.3 & 19.8 & 94.5 \\
\hline $\begin{array}{l}\text { BU0755- } \\
76\end{array}$ & 402 & 60693 & 1.9 & $\begin{array}{r}9.347 \\
3\end{array}$ & 0.8 & 4.0656 & 1.3 & $\begin{array}{r}0.275 \\
6\end{array}$ & $\begin{array}{r}1 . \\
0\end{array}$ & $\begin{array}{r}0.7 \\
8\end{array}$ & $\begin{array}{r}1569 . \\
2\end{array}$ & 14.5 & 1647.4 & 10.8 & $\begin{array}{r}1748 . \\
6\end{array}$ & 15.2 & 1748.6 & 15.2 & 89.7 \\
\hline $\begin{array}{l}\text { BU0755- } \\
45\end{array}$ & 632 & 62742 & 1.6 & $\begin{array}{r}9.337 \\
7\end{array}$ & 1.3 & 3.4932 & 4.4 & $\begin{array}{r}0.236 \\
6\end{array}$ & $\begin{array}{r}4 . \\
2\end{array}$ & $\begin{array}{r}0.9 \\
5\end{array}$ & $\begin{array}{r}1368 . \\
9\end{array}$ & 52.0 & 1525.7 & 35.0 & $\begin{array}{r}1750 . \\
5\end{array}$ & 24.5 & 1750.5 & 24.5 & 78.2 \\
\hline
\end{tabular}




\begin{tabular}{|c|c|c|c|c|c|c|c|c|c|c|c|c|c|c|c|c|c|c|c|}
\hline $\begin{array}{l}\text { BU0755- } \\
73\end{array}$ & 662 & 36849 & 2.4 & $\begin{array}{r}9.329 \\
5 \\
\end{array}$ & 1.7 & 3.9306 & 2.8 & $\begin{array}{r}0.266 \\
0 \\
\end{array}$ & $\begin{array}{r}2 . \\
2 \\
\end{array}$ & $\begin{array}{r}0.8 \\
0 \\
\end{array}$ & $\begin{array}{r}1520 . \\
3 \\
\end{array}$ & 30.2 & 1620.0 & 22.5 & $\begin{array}{r}1752 . \\
1 \\
\end{array}$ & 30.2 & 1752.1 & 30.2 & 86.8 \\
\hline $\begin{array}{l}\text { BU0755- } \\
56\end{array}$ & 511 & 72309 & 2.6 & $\begin{array}{r}9.328 \\
1\end{array}$ & 1.3 & 4.3914 & 1.4 & $\begin{array}{r}0.297 \\
1\end{array}$ & $\begin{array}{r}0 . \\
5\end{array}$ & $\begin{array}{r}0.3 \\
6\end{array}$ & $\begin{array}{r}1676 . \\
9\end{array}$ & 7.4 & 1710.7 & 11.4 & $\begin{array}{r}1752 . \\
4\end{array}$ & 23.6 & 1752.4 & 23.6 & 95.7 \\
\hline $\begin{array}{l}\text { BU0755- } \\
65\end{array}$ & 150 & 31737 & 1.9 & $\begin{array}{r}9.326 \\
5\end{array}$ & 1.6 & 4.3234 & 2.1 & $\begin{array}{r}0.292 \\
4\end{array}$ & $\begin{array}{r}1 . \\
4\end{array}$ & $\begin{array}{r}0.6 \\
5\end{array}$ & $\begin{array}{r}1653 . \\
7\end{array}$ & 20.1 & 1697.8 & 17.5 & $\begin{array}{r}1752 . \\
7\end{array}$ & 29.5 & 1752.7 & 29.5 & 94.4 \\
\hline $\begin{array}{l}\text { BU0755- } \\
11\end{array}$ & $\begin{array}{r}112 \\
6 \\
\end{array}$ & $\begin{array}{r}19305 \\
6 \\
\end{array}$ & 3.8 & $\begin{array}{r}9.294 \\
0 \\
\end{array}$ & 1.0 & 4.5120 & 3.5 & $\begin{array}{r}0.304 \\
1 \\
\end{array}$ & $\begin{array}{r}3 . \\
4\end{array}$ & $\begin{array}{r}0.9 \\
6 \\
\end{array}$ & $\begin{array}{r}1711 . \\
8\end{array}$ & 51.0 & 1733.2 & 29.4 & $\begin{array}{r}1759 . \\
1\end{array}$ & 18.3 & 1759.1 & 18.3 & 97.3 \\
\hline $\begin{array}{l}\text { BU0755- } \\
86\end{array}$ & 681 & 58224 & 1.7 & $\begin{array}{r}9.293 \\
6 \\
\end{array}$ & 1.1 & 4.2687 & 2.2 & $\begin{array}{r}0.287 \\
7 \\
\end{array}$ & $\begin{array}{r}1 . \\
9\end{array}$ & $\begin{array}{r}0.8 \\
6 \\
\end{array}$ & $\begin{array}{r}1630 . \\
2 \\
\end{array}$ & 27.2 & 1687.4 & 18.0 & $\begin{array}{r}1759 . \\
2 \\
\end{array}$ & 20.1 & 1759.2 & 20.1 & 92.7 \\
\hline BU0755-3 & $\begin{array}{r}100 \\
1 \\
\end{array}$ & $\begin{array}{r}13021 \\
2 \\
\end{array}$ & 1.2 & $\begin{array}{r}9.286 \\
6 \\
\end{array}$ & 1.8 & 4.5931 & 2.4 & $\begin{array}{r}0.309 \\
4 \\
\end{array}$ & $\begin{array}{r}1 . \\
6\end{array}$ & $\begin{array}{r}0.6 \\
5 \\
\end{array}$ & $\begin{array}{r}1737 . \\
5 \\
\end{array}$ & 23.9 & 1748.0 & 20.1 & $\begin{array}{r}1760 . \\
5\end{array}$ & 33.5 & 1760.5 & 33.5 & 98.7 \\
\hline $\begin{array}{l}\text { BU0755- } \\
28\end{array}$ & 461 & 85266 & 1.0 & $\begin{array}{r}9.277 \\
5\end{array}$ & 1.7 & 4.6667 & 2.3 & $\begin{array}{r}0.314 \\
0\end{array}$ & $\begin{array}{r}1 . \\
6\end{array}$ & $\begin{array}{r}0.6 \\
9\end{array}$ & $\begin{array}{r}1760 . \\
4\end{array}$ & 24.3 & 1761.3 & 19.2 & $\begin{array}{r}1762 . \\
3\end{array}$ & 30.3 & 1762.3 & 30.3 & 99.9 \\
\hline $\begin{array}{l}\text { BU0755- } \\
27\end{array}$ & 203 & 27615 & 1.0 & $\begin{array}{r}9.271 \\
7 \\
\end{array}$ & 2.0 & 4.1301 & 4.0 & $\begin{array}{r}0.277 \\
7 \\
\end{array}$ & $\begin{array}{r}3 . \\
5\end{array}$ & $\begin{array}{r}0.8 \\
7 \\
\end{array}$ & $\begin{array}{r}1579 . \\
9 \\
\end{array}$ & 48.6 & 1660.3 & 32.6 & $\begin{array}{r}1763 . \\
5 \\
\end{array}$ & 35.8 & 1763.5 & 35.8 & 89.6 \\
\hline $\begin{array}{l}\text { BU0755- } \\
69\end{array}$ & $\begin{array}{r}114 \\
6\end{array}$ & $\begin{array}{r}13483 \\
8\end{array}$ & 1.3 & $\begin{array}{r}9.268 \\
0\end{array}$ & 2.5 & 3.9411 & 3.0 & $\begin{array}{r}0.264 \\
9\end{array}$ & $\begin{array}{r}1 . \\
8\end{array}$ & $\begin{array}{r}0.5 \\
8\end{array}$ & $\begin{array}{r}1514 . \\
9\end{array}$ & 23.6 & 1622.2 & 24.6 & $\begin{array}{r}1764 . \\
2\end{array}$ & 45.3 & 1764.2 & 45.3 & 85.9 \\
\hline $\begin{array}{l}\text { BU0755- } \\
10\end{array}$ & 192 & 41307 & 1.5 & $\begin{array}{r}9.262 \\
6 \\
\end{array}$ & 2.2 & 4.8124 & 2.8 & $\begin{array}{r}0.323 \\
3 \\
\end{array}$ & $\begin{array}{r}1 . \\
7 \\
\end{array}$ & $\begin{array}{r}0.6 \\
1 \\
\end{array}$ & $\begin{array}{r}1805 . \\
8 \\
\end{array}$ & 27.2 & 1787.1 & 23.8 & $\begin{array}{r}1765 . \\
3 \\
\end{array}$ & 40.9 & 1765.3 & 40.9 & $\begin{array}{r}102 . \\
3 \\
\end{array}$ \\
\hline $\begin{array}{l}\text { BU0755- } \\
37\end{array}$ & 743 & 74610 & 1.3 & $\begin{array}{r}9.214 \\
8\end{array}$ & 4.5 & 4.0202 & 6.0 & $\begin{array}{r}0.268 \\
7\end{array}$ & $\begin{array}{r}4 . \\
0\end{array}$ & $\begin{array}{r}0.6 \\
7\end{array}$ & $\begin{array}{r}1534 . \\
1\end{array}$ & 54.9 & 1638.3 & 49.1 & $\begin{array}{r}1774 . \\
7\end{array}$ & 82.2 & 1774.7 & 82.2 & 86.4 \\
\hline $\begin{array}{l}\text { BU0755- } \\
33\end{array}$ & 328 & 37038 & 2.2 & $\begin{array}{r}9.191 \\
0\end{array}$ & 3.4 & 4.4454 & 3.7 & $\begin{array}{r}0.296 \\
3\end{array}$ & $\begin{array}{r}1 . \\
5\end{array}$ & $\begin{array}{r}0.4 \\
0\end{array}$ & $\begin{array}{r}1673 . \\
1\end{array}$ & 21.8 & 1720.9 & 30.7 & $\begin{array}{r}1779 . \\
4\end{array}$ & 61.9 & 1779.4 & 61.9 & 94.0 \\
\hline $\begin{array}{l}\text { BU0755- } \\
74\end{array}$ & 991 & $\begin{array}{r}13848 \\
9 \\
\end{array}$ & 0.9 & $\begin{array}{r}9.181 \\
3 \\
\end{array}$ & 1.6 & 4.1183 & 2.5 & $\begin{array}{r}0.274 \\
2 \\
\end{array}$ & $\begin{array}{r}1 . \\
9\end{array}$ & $\begin{array}{r}0.7 \\
6 \\
\end{array}$ & $\begin{array}{r}1562 . \\
3\end{array}$ & 26.6 & 1657.9 & 20.6 & $\begin{array}{r}1781 . \\
4\end{array}$ & 29.9 & 1781.4 & 29.9 & 87.7 \\
\hline $\begin{array}{l}\text { BU0755- } \\
36\end{array}$ & 305 & 29253 & 1.5 & $\begin{array}{r}9.167 \\
8 \\
\end{array}$ & 1.9 & 4.0813 & 4.2 & $\begin{array}{r}0.271 \\
4 \\
\end{array}$ & $\begin{array}{l}3 . \\
7 \\
\end{array}$ & $\begin{array}{r}0.9 \\
0 \\
\end{array}$ & $\begin{array}{r}1547 . \\
7 \\
\end{array}$ & 51.5 & 1650.6 & 34.0 & $\begin{array}{r}1784 . \\
0 \\
\end{array}$ & 33.7 & 1784.0 & 33.7 & 86.8 \\
\hline BU0755-2 & 292 & 41046 & 1.8 & $\begin{array}{r}9.037 \\
4 \\
\end{array}$ & 1.5 & 4.5281 & 3.6 & $\begin{array}{r}0.296 \\
8 \\
\end{array}$ & $\begin{array}{r}3 . \\
2\end{array}$ & $\begin{array}{r}0.9 \\
1 \\
\end{array}$ & $\begin{array}{r}1675 . \\
4 \\
\end{array}$ & 47.8 & 1736.1 & 29.6 & $\begin{array}{r}1810 . \\
1 \\
\end{array}$ & 26.9 & 1810.1 & 26.9 & 92.6 \\
\hline $\begin{array}{l}\text { BU0755- } \\
21\end{array}$ & 69 & 13890 & 1.3 & $\begin{array}{r}9.013 \\
5\end{array}$ & 1.8 & 4.9154 & 3.3 & $\begin{array}{r}0.321 \\
3\end{array}$ & $\begin{array}{r}2 . \\
8\end{array}$ & $\begin{array}{r}0.8 \\
4\end{array}$ & $\begin{array}{r}1796 . \\
2\end{array}$ & 43.1 & 1804.9 & 27.5 & $\begin{array}{r}1814 . \\
9\end{array}$ & 31.8 & 1814.9 & 31.8 & 99.0 \\
\hline $\begin{array}{l}\text { BU0755- } \\
89\end{array}$ & 411 & 31977 & 1.7 & $\begin{array}{r}8.967 \\
4 \\
\end{array}$ & 1.7 & 3.4836 & 8.1 & $\begin{array}{r}0.226 \\
6 \\
\end{array}$ & $\begin{array}{r}8 . \\
0 \\
\end{array}$ & $\begin{array}{r}0.9 \\
8 \\
\end{array}$ & $\begin{array}{r}1316 . \\
5 \\
\end{array}$ & 94.9 & 1523.5 & 64.3 & $\begin{array}{r}1824 . \\
2 \\
\end{array}$ & 30.5 & 1824.2 & 30.5 & 72.2 \\
\hline $\begin{array}{l}\text { BU0755- } \\
17\end{array}$ & 413 & 51081 & 0.8 & $\begin{array}{r}8.931 \\
6 \\
\end{array}$ & 2.5 & 4.1195 & 3.4 & $\begin{array}{r}0.266 \\
8 \\
\end{array}$ & $\begin{array}{r}2 . \\
4 \\
\end{array}$ & $\begin{array}{r}0.6 \\
9 \\
\end{array}$ & $\begin{array}{r}1524 . \\
8 \\
\end{array}$ & 32.0 & 1658.2 & 28.1 & $\begin{array}{r}1831 . \\
5 \\
\end{array}$ & 45.3 & 1831.5 & 45.3 & 83.3 \\
\hline $\begin{array}{l}\text { BU0755- } \\
14\end{array}$ & 200 & 37824 & 1.8 & $\begin{array}{r}8.248 \\
1 \\
\end{array}$ & 1.1 & 5.9725 & 3.4 & $\begin{array}{r}0.357 \\
3 \\
\end{array}$ & $\begin{array}{r}3 . \\
2 \\
\end{array}$ & $\begin{array}{r}0.9 \\
4 \\
\end{array}$ & $\begin{array}{r}1969 . \\
3 \\
\end{array}$ & 53.8 & 1971.9 & 29.3 & $\begin{array}{r}1974 . \\
5 \\
\end{array}$ & 20.0 & 1974.5 & 20.0 & 99.7 \\
\hline $\begin{array}{l}\text { BU0755- } \\
90\end{array}$ & 588 & 68880 & 2.3 & $\begin{array}{r}7.233 \\
6\end{array}$ & 1.2 & 6.3661 & 2.8 & $\begin{array}{r}0.334 \\
0\end{array}$ & $\begin{array}{r}2 . \\
6\end{array}$ & $\begin{array}{r}0.9 \\
1\end{array}$ & $\begin{array}{r}1857 . \\
7\end{array}$ & 41.6 & 2027.6 & 24.8 & $\begin{array}{r}2205 . \\
3\end{array}$ & 20.1 & 2205.3 & 20.1 & 84.2 \\
\hline $\begin{array}{l}\text { BU0755- } \\
93\end{array}$ & 336 & 66012 & 5.4 & $\begin{array}{r}7.074 \\
8 \\
\end{array}$ & 2.0 & 7.4392 & 2.3 & $\begin{array}{r}0.381 \\
7 \\
\end{array}$ & $\begin{array}{r}1 . \\
3\end{array}$ & $\begin{array}{r}0.5 \\
5\end{array}$ & $\begin{array}{r}2084 . \\
3\end{array}$ & 22.8 & 2165.7 & 21.0 & $\begin{array}{r}2243 . \\
8\end{array}$ & 33.9 & 2243.8 & 33.9 & 92.9 \\
\hline $\begin{array}{l}\text { BU0755- } \\
38\end{array}$ & 865 & 89772 & 10.7 & $\begin{array}{r}6.996 \\
8\end{array}$ & 4.9 & 6.5380 & 4.9 & $\begin{array}{r}0.331 \\
8\end{array}$ & $\begin{array}{r}0 . \\
9\end{array}$ & $\begin{array}{r}0.1 \\
9\end{array}$ & $\begin{array}{r}1847 . \\
0\end{array}$ & 14.9 & 2051.0 & 43.5 & $\begin{array}{r}2262 . \\
9\end{array}$ & 83.7 & 2262.9 & 83.7 & 81.6 \\
\hline $\begin{array}{l}\text { BU0755- } \\
46\end{array}$ & 312 & 48606 & 2.2 & $\begin{array}{r}6.483 \\
9\end{array}$ & 1.3 & 7.4424 & 3.7 & $\begin{array}{r}0.350 \\
0\end{array}$ & $\begin{array}{r}3 . \\
5\end{array}$ & $\begin{array}{r}0.9 \\
3\end{array}$ & $\begin{array}{r}1934 . \\
5\end{array}$ & 58.5 & 2166.1 & 33.6 & $\begin{array}{r}2393 . \\
3\end{array}$ & 22.8 & 2393.3 & 22.8 & 80.8 \\
\hline $\begin{array}{l}\text { BU0755- } \\
51\end{array}$ & 852 & $\begin{array}{r}16987 \\
5 \\
\end{array}$ & 2.1 & $\begin{array}{r}6.380 \\
5 \\
\end{array}$ & 1.8 & 9.0448 & 2.2 & $\begin{array}{r}0.418 \\
6 \\
\end{array}$ & $\begin{array}{r}1 . \\
3 \\
\end{array}$ & $\begin{array}{r}0.5 \\
7 \\
\end{array}$ & $\begin{array}{r}2253 . \\
9 \\
\end{array}$ & 23.8 & 2342.5 & 20.0 & $\begin{array}{r}2420 . \\
6 \\
\end{array}$ & 30.4 & 2420.6 & 30.4 & 93.1 \\
\hline BU0755- & 122 & 16885 & 5.6 & 6.314 & 2.1 & 7.4440 & 3.0 & 0.340 & 2. & 0.7 & 1891. & 35.1 & 2166.3 & 27.0 & 2438. & 36.1 & 2438.3 & 36.1 & 77.6 \\
\hline
\end{tabular}




\begin{tabular}{|c|c|c|c|c|c|c|c|c|c|c|c|c|c|c|c|c|c|c|c|}
\hline 66 & 6 & 5 & & 5 & & & & 9 & 1 & 1 & 1 & & & & 3 & & & & \\
\hline $\begin{array}{l}\text { BU0755- } \\
101\end{array}$ & 124 & 29958 & 1.5 & $\begin{array}{r}6.182 \\
8 \\
\end{array}$ & 1.3 & 9.3117 & 3.3 & $\begin{array}{r}0.417 \\
6 \\
\end{array}$ & $\begin{array}{r}3 . \\
0\end{array}$ & $\begin{array}{r}0.9 \\
2 \\
\end{array}$ & $\begin{array}{r}2249 . \\
4 \\
\end{array}$ & 57.0 & 2369.2 & 29.8 & $\begin{array}{r}2473 . \\
9 \\
\end{array}$ & 21.1 & 2473.9 & 21.1 & 90.9 \\
\hline $\begin{array}{l}\text { BU0755- } \\
94\end{array}$ & 238 & 54609 & 3.2 & $\begin{array}{r}6.018 \\
2 \\
\end{array}$ & 1.8 & 9.5559 & 2.1 & $\begin{array}{r}0.417 \\
1 \\
\end{array}$ & $\begin{array}{r}1 . \\
2 \\
\end{array}$ & $\begin{array}{r}0.5 \\
7 \\
\end{array}$ & $\begin{array}{r}2247 . \\
3 \\
\end{array}$ & 23.1 & 2392.9 & 19.8 & $\begin{array}{r}2519 . \\
3 \\
\end{array}$ & 29.7 & 2519.3 & 29.7 & 89.2 \\
\hline $\begin{array}{l}\text { BU0755- } \\
53\end{array}$ & 299 & 73377 & 1.7 & $\begin{array}{r}6.000 \\
0 \\
\end{array}$ & 2.7 & $\begin{array}{r}10.044 \\
2 \\
\end{array}$ & 3.7 & $\begin{array}{r}0.437 \\
1 \\
\end{array}$ & $\begin{array}{r}2 . \\
5\end{array}$ & $\begin{array}{r}0.6 \\
8 \\
\end{array}$ & $\begin{array}{r}2337 . \\
6 \\
\end{array}$ & 49.6 & 2438.9 & 34.4 & $\begin{array}{r}2524 . \\
4\end{array}$ & 45.9 & 2524.4 & 45.9 & 92.6 \\
\hline $\begin{array}{l}\text { BU0755- } \\
72\end{array}$ & 377 & 67599 & 1.4 & $\begin{array}{r}5.940 \\
5 \\
\end{array}$ & 1.5 & 9.2756 & 1.8 & $\begin{array}{r}0.399 \\
6 \\
\end{array}$ & $\begin{array}{r}0 . \\
9\end{array}$ & $\begin{array}{r}0.5 \\
3\end{array}$ & $\begin{array}{r}2167 . \\
3\end{array}$ & 16.9 & 2365.6 & 16.1 & $\begin{array}{r}2541 . \\
2\end{array}$ & 25.0 & 2541.2 & 25.0 & 85.3 \\
\hline $\begin{array}{l}\text { BU0755- } \\
48\end{array}$ & 700 & $\begin{array}{r}21762 \\
6 \\
\end{array}$ & 2.6 & $\begin{array}{r}4.242 \\
8 \\
\end{array}$ & 2.3 & $\begin{array}{r}19.569 \\
2 \\
\end{array}$ & 2.6 & $\begin{array}{r}0.602 \\
2 \\
\end{array}$ & $\begin{array}{l}1 . \\
3 \\
\end{array}$ & $\begin{array}{r}0.4 \\
8 \\
\end{array}$ & $\begin{array}{r}3038 . \\
6 \\
\end{array}$ & 31.0 & 3070.3 & 25.5 & $\begin{array}{r}3091 . \\
1 \\
\end{array}$ & 36.9 & 3091.1 & 36.9 & 98.3 \\
\hline $\begin{array}{l}\text { BU0818- } \\
87\end{array}$ & 425 & 16020 & 0.9 & $\begin{array}{r}14.46 \\
05 \\
\end{array}$ & 1.9 & 1.1951 & 2.4 & $\begin{array}{r}0.125 \\
3 \\
\end{array}$ & $\begin{array}{r}1 . \\
4 \\
\end{array}$ & $\begin{array}{r}0.5 \\
8 \\
\end{array}$ & 761.2 & 9.9 & 798.3 & 13.2 & 903.3 & 39.9 & 761.2 & 9.9 & 84.3 \\
\hline BU0818-3 & $\begin{array}{r}134 \\
8 \\
\end{array}$ & 32804 & 12.0 & $\begin{array}{r}13.90 \\
69 \\
\end{array}$ & 3.1 & 1.2969 & 3.1 & $\begin{array}{r}0.130 \\
8 \\
\end{array}$ & $\begin{array}{r}0 . \\
5\end{array}$ & $\begin{array}{r}0.1 \\
6 \\
\end{array}$ & 792.5 & 3.7 & 844.4 & 18.0 & 983.3 & 63.2 & 792.5 & 3.7 & 80.6 \\
\hline $\begin{array}{l}\text { BU0818- } \\
29\end{array}$ & 535 & 32150 & 12.3 & $\begin{array}{r}14.64 \\
41 \\
\end{array}$ & 1.7 & 1.2423 & 2.0 & $\begin{array}{r}0.131 \\
9 \\
\end{array}$ & $\begin{array}{r}1 . \\
0\end{array}$ & $\begin{array}{r}0.5 \\
0 \\
\end{array}$ & 799.0 & 7.3 & 819.9 & 11.0 & 877.3 & 35.2 & 799.0 & 7.3 & 91.1 \\
\hline $\begin{array}{l}\text { BU0818- } \\
60\end{array}$ & 636 & 33220 & 2.0 & $\begin{array}{r}14.74 \\
03 \\
\end{array}$ & 2.3 & 1.2450 & 3.5 & $\begin{array}{r}0.133 \\
1 \\
\end{array}$ & $\begin{array}{r}2 . \\
7 \\
\end{array}$ & $\begin{array}{r}0.7 \\
7\end{array}$ & 805.5 & 20.5 & 821.1 & 19.9 & 863.7 & 47.3 & 805.5 & 20.5 & 93.3 \\
\hline $\begin{array}{l}\text { BU0818- } \\
21\end{array}$ & 541 & 16358 & 3.4 & $\begin{array}{r}14.81 \\
12 \\
\end{array}$ & 2.4 & 1.2495 & 2.4 & $\begin{array}{r}0.134 \\
2 \\
\end{array}$ & $\begin{array}{r}0 . \\
5 \\
\end{array}$ & $\begin{array}{r}0.2 \\
0 \\
\end{array}$ & 811.9 & 3.8 & 823.2 & 13.8 & 853.8 & 49.8 & 811.9 & 3.8 & 95.1 \\
\hline $\begin{array}{l}\text { BU0818- } \\
67\end{array}$ & $\begin{array}{r}195 \\
2 \\
\end{array}$ & 83088 & 21.9 & $\begin{array}{r}14.04 \\
53 \\
\end{array}$ & 3.8 & 1.3257 & 3.9 & $\begin{array}{r}0.135 \\
0 \\
\end{array}$ & $\begin{array}{r}0 . \\
9 \\
\end{array}$ & $\begin{array}{r}0.2 \\
3 \\
\end{array}$ & 816.6 & 6.7 & 857.0 & 22.6 & 963.1 & 77.6 & 816.6 & 6.7 & 84.8 \\
\hline $\begin{array}{l}\text { BU0818- } \\
17\end{array}$ & 325 & 19112 & 1.1 & $\begin{array}{r}13.56 \\
20 \\
\end{array}$ & 2.3 & 1.4178 & 2.8 & $\begin{array}{r}0.139 \\
5 \\
\end{array}$ & $\begin{array}{r}1 . \\
5\end{array}$ & $\begin{array}{r}0.5 \\
4 \\
\end{array}$ & 841.6 & 11.8 & 896.5 & 16.5 & $\begin{array}{r}1034 . \\
2 \\
\end{array}$ & 46.9 & 841.6 & 11.8 & 81.4 \\
\hline $\begin{array}{l}\text { BU0818- } \\
98\end{array}$ & 174 & 5554 & 2.7 & $\begin{array}{r}13.51 \\
62 \\
\end{array}$ & 2.3 & 1.4301 & 4.2 & $\begin{array}{r}0.140 \\
2 \\
\end{array}$ & $\begin{array}{r}3 . \\
5\end{array}$ & $\begin{array}{r}0.8 \\
4\end{array}$ & 845.8 & 27.9 & 901.6 & 25.2 & $\begin{array}{r}1041 . \\
0\end{array}$ & 46.7 & 845.8 & 27.9 & 81.2 \\
\hline BU0818-4 & 179 & 11900 & 1.4 & $\begin{array}{r}14.56 \\
92 \\
\end{array}$ & 2.7 & 1.3577 & 2.8 & $\begin{array}{r}0.143 \\
5 \\
\end{array}$ & $\begin{array}{r}0 . \\
8 \\
\end{array}$ & $\begin{array}{r}0.2 \\
8 \\
\end{array}$ & 864.2 & 6.4 & 870.9 & 16.4 & 887.9 & 55.6 & 864.2 & 6.4 & 97.3 \\
\hline $\begin{array}{l}\text { BU0818- } \\
23\end{array}$ & 778 & 58982 & 18.1 & $\begin{array}{r}14.02 \\
91 \\
\end{array}$ & 1.6 & 1.4113 & 3.1 & $\begin{array}{r}0.143 \\
6 \\
\end{array}$ & $\begin{array}{l}2 . \\
7 \\
\end{array}$ & $\begin{array}{r}0.8 \\
7 \\
\end{array}$ & 865.0 & 21.9 & 893.7 & 18.6 & 965.5 & 31.6 & 865.0 & 21.9 & 89.6 \\
\hline $\begin{array}{l}\text { BU0818- } \\
25\end{array}$ & 300 & 8150 & 2.5 & $\begin{array}{r}13.83 \\
97 \\
\end{array}$ & 3.1 & 1.4331 & 3.3 & $\begin{array}{r}0.143 \\
9 \\
\end{array}$ & $\begin{array}{r}1 . \\
1 \\
\end{array}$ & $\begin{array}{r}0.3 \\
3 \\
\end{array}$ & 866.4 & 8.9 & 902.9 & 19.9 & 993.1 & 63.8 & 866.4 & 8.9 & 87.2 \\
\hline $\begin{array}{l}\text { BU0818- } \\
90\end{array}$ & 308 & 13578 & 1.4 & $\begin{array}{r}14.67 \\
77 \\
\end{array}$ & 1.0 & 1.3559 & 1.3 & $\begin{array}{r}0.144 \\
3 \\
\end{array}$ & $\begin{array}{r}0 . \\
9 \\
\end{array}$ & $\begin{array}{r}0.6 \\
8 \\
\end{array}$ & 869.1 & 7.4 & 870.1 & 7.8 & 872.5 & 20.4 & 869.1 & 7.4 & 99.6 \\
\hline $\begin{array}{l}\text { BU0818- } \\
33\end{array}$ & 774 & 44676 & 2.8 & $\begin{array}{r}14.08 \\
85 \\
\end{array}$ & 1.7 & 1.4273 & 1.8 & $\begin{array}{r}0.145 \\
8 \\
\end{array}$ & $\begin{array}{l}0 . \\
7 \\
\end{array}$ & $\begin{array}{r}0.3 \\
9 \\
\end{array}$ & 877.6 & 5.8 & 900.4 & 10.9 & 956.8 & 34.4 & 877.6 & 5.8 & 91.7 \\
\hline $\begin{array}{l}\text { BU0818- } \\
61\end{array}$ & 385 & 26568 & 3.2 & $\begin{array}{r}13.97 \\
78 \\
\end{array}$ & 2.7 & 1.4420 & 2.8 & $\begin{array}{r}0.146 \\
2 \\
\end{array}$ & $\begin{array}{r}0 . \\
8\end{array}$ & $\begin{array}{r}0.2 \\
8 \\
\end{array}$ & 879.6 & 6.5 & 906.6 & 17.0 & 973.0 & 55.5 & 879.6 & 6.5 & 90.4 \\
\hline $\begin{array}{l}\text { BU0818- } \\
89\end{array}$ & 257 & 17734 & 1.6 & $\begin{array}{r}14.76 \\
33 \\
\end{array}$ & 1.9 & 1.3888 & 2.4 & $\begin{array}{r}0.148 \\
7 \\
\end{array}$ & $\begin{array}{r}1 . \\
5\end{array}$ & $\begin{array}{r}0.6 \\
3 \\
\end{array}$ & 893.7 & 12.6 & 884.2 & 14.2 & 860.5 & 38.9 & 893.7 & 12.6 & $\begin{array}{r}103 . \\
9 \\
\end{array}$ \\
\hline BU0818-8 & 683 & 41246 & 8.8 & $\begin{array}{r}14.28 \\
86 \\
\end{array}$ & 2.1 & 1.4453 & 2.7 & $\begin{array}{r}0.149 \\
8 \\
\end{array}$ & $\begin{array}{r}1 . \\
8\end{array}$ & $\begin{array}{r}0.6 \\
6 \\
\end{array}$ & 899.7 & 14.9 & 907.9 & 16.3 & 928.0 & 42.1 & 899.7 & 14.9 & 97.0 \\
\hline $\begin{array}{l}\text { BU0818- } \\
47\end{array}$ & 390 & 26518 & 2.3 & $\begin{array}{r}14.46 \\
07\end{array}$ & 1.4 & 1.4413 & 1.5 & $\begin{array}{r}0.151 \\
2\end{array}$ & $\begin{array}{r}0 . \\
6\end{array}$ & $\begin{array}{r}0.4 \\
0\end{array}$ & 907.5 & 5.2 & 906.3 & 9.2 & 903.3 & 29.1 & 907.5 & 5.2 & $\begin{array}{r}100 . \\
5\end{array}$ \\
\hline $\begin{array}{l}\text { BU0818- } \\
103\end{array}$ & 147 & 8868 & 2.0 & $\begin{array}{r}14.37 \\
18 \\
\end{array}$ & 1.5 & 1.4503 & 2.4 & $\begin{array}{r}0.151 \\
2 \\
\end{array}$ & $\begin{array}{r}1 . \\
8\end{array}$ & $\begin{array}{r}0.7 \\
8 \\
\end{array}$ & 907.5 & 15.5 & 910.0 & 14.1 & 916.0 & 30.4 & 907.5 & 15.5 & 99.1 \\
\hline
\end{tabular}




\begin{tabular}{|c|c|c|c|c|c|c|c|c|c|c|c|c|c|c|c|c|c|c|c|}
\hline $\begin{array}{l}\text { BU0818- } \\
48\end{array}$ & 198 & 14072 & 0.9 & $\begin{array}{r}14.05 \\
73 \\
\end{array}$ & 1.3 & 1.4917 & 1.6 & $\begin{array}{r}0.152 \\
1 \\
\end{array}$ & $\begin{array}{r}0 . \\
9\end{array}$ & $\begin{array}{r}0.5 \\
7 \\
\end{array}$ & 912.6 & 8.0 & 927.0 & 10.0 & 961.4 & 27.4 & 912.6 & 8.0 & 94.9 \\
\hline $\begin{array}{l}\text { BU0818- } \\
38\end{array}$ & 76 & 7454 & 0.6 & $\begin{array}{r}14.19 \\
38\end{array}$ & 3.2 & 1.4892 & 3.7 & $\begin{array}{r}0.153 \\
3\end{array}$ & $\begin{array}{r}1 . \\
9\end{array}$ & $\begin{array}{r}0.5 \\
0\end{array}$ & 919.4 & 15.9 & 926.0 & 22.3 & 941.6 & 65.0 & 919.4 & 15.9 & 97.6 \\
\hline $\begin{array}{l}\text { BU0818- } \\
18\end{array}$ & 291 & 17274 & 1.1 & $\begin{array}{r}13.95 \\
22\end{array}$ & 2.7 & 1.5473 & 2.8 & $\begin{array}{r}0.156 \\
6\end{array}$ & $\begin{array}{r}0 . \\
8\end{array}$ & $\begin{array}{r}0.3 \\
0\end{array}$ & 937.7 & 7.2 & 949.4 & 17.3 & 976.7 & 54.6 & 937.7 & 7.2 & 96.0 \\
\hline $\begin{array}{l}\text { BU0818- } \\
73\end{array}$ & 684 & 32394 & 2.9 & $\begin{array}{r}13.73 \\
82 \\
\end{array}$ & 1.2 & 1.5797 & 2.1 & $\begin{array}{r}0.157 \\
4 \\
\end{array}$ & $\begin{array}{r}1 . \\
7 \\
\end{array}$ & $\begin{array}{r}0.8 \\
1 \\
\end{array}$ & 942.3 & 14.6 & 962.3 & 12.8 & $\begin{array}{r}1008 . \\
1\end{array}$ & 24.4 & 942.3 & 14.6 & 93.5 \\
\hline $\begin{array}{l}\text { BU0818- } \\
96\end{array}$ & $\begin{array}{r}167 \\
7 \\
\end{array}$ & 79340 & 11.8 & $\begin{array}{r}13.67 \\
08 \\
\end{array}$ & 0.9 & 1.5911 & 1.0 & $\begin{array}{r}0.157 \\
8 \\
\end{array}$ & $\begin{array}{c}0 . \\
5 \\
\end{array}$ & $\begin{array}{r}0.5 \\
1 \\
\end{array}$ & 944.3 & 4.7 & 966.7 & 6.5 & $\begin{array}{r}1018 . \\
1 \\
\end{array}$ & 18.0 & 944.3 & 4.7 & 92.8 \\
\hline $\begin{array}{l}\text { BU0818- } \\
44\end{array}$ & 533 & 42592 & 3.1 & $\begin{array}{r}13.89 \\
14 \\
\end{array}$ & 1.3 & 1.5989 & 1.6 & $\begin{array}{r}0.161 \\
1 \\
\end{array}$ & $\begin{array}{r}1 . \\
0\end{array}$ & $\begin{array}{r}0.6 \\
1 \\
\end{array}$ & 962.8 & 8.6 & 969.8 & 9.9 & 985.6 & 25.5 & 962.8 & 8.6 & 97.7 \\
\hline $\begin{array}{l}\text { BU0818- } \\
32\end{array}$ & 934 & 55436 & 13.4 & $\begin{array}{r}13.84 \\
64 \\
\end{array}$ & 2.1 & 1.6065 & 2.2 & $\begin{array}{r}0.161 \\
3 \\
\end{array}$ & $\begin{array}{r}0 . \\
6 \\
\end{array}$ & $\begin{array}{r}0.2 \\
7 \\
\end{array}$ & 964.2 & 5.4 & 972.8 & 13.8 & 992.2 & 43.1 & 964.2 & 5.4 & 97.2 \\
\hline $\begin{array}{l}\text { BU0818- } \\
77\end{array}$ & 536 & 33894 & 6.5 & $\begin{array}{r}14.03 \\
70 \\
\end{array}$ & 0.6 & 1.5869 & 2.1 & $\begin{array}{r}0.161 \\
6\end{array}$ & $\begin{array}{r}2 . \\
0\end{array}$ & $\begin{array}{r}0.9 \\
6\end{array}$ & 965.4 & 17.6 & 965.1 & 12.8 & 964.3 & 12.3 & 965.4 & 17.6 & $\begin{array}{r}100 . \\
1\end{array}$ \\
\hline $\begin{array}{l}\text { BU0818- } \\
88\end{array}$ & 241 & 17148 & 1.0 & $\begin{array}{r}13.81 \\
39 \\
\end{array}$ & 1.1 & 1.6520 & 1.3 & $\begin{array}{r}0.165 \\
5 \\
\end{array}$ & $\begin{array}{r}0 . \\
6 \\
\end{array}$ & $\begin{array}{r}0.5 \\
0 \\
\end{array}$ & 987.3 & 5.8 & 990.3 & 7.9 & 997.0 & 22.1 & 987.3 & 5.8 & 99.0 \\
\hline $\begin{array}{l}\text { BU0818- } \\
94\end{array}$ & 362 & 19184 & 3.0 & $\begin{array}{r}13.53 \\
78 \\
\end{array}$ & 1.7 & 1.6958 & 2.0 & $\begin{array}{r}0.166 \\
5 \\
\end{array}$ & $\begin{array}{r}1 . \\
0\end{array}$ & $\begin{array}{r}0.4 \\
8 \\
\end{array}$ & 992.8 & 8.7 & 1007.0 & 12.7 & $\begin{array}{r}1037 . \\
8 \\
\end{array}$ & 35.2 & 992.8 & 8.7 & 95.7 \\
\hline $\begin{array}{l}\text { BU0818- } \\
86\end{array}$ & 574 & 35990 & 9.1 & $\begin{array}{r}13.81 \\
17\end{array}$ & 1.6 & 1.7031 & 1.9 & $\begin{array}{r}0.170 \\
6\end{array}$ & $\begin{array}{r}1 . \\
1\end{array}$ & $\begin{array}{r}0.5 \\
8\end{array}$ & $\begin{array}{r}1015 . \\
4\end{array}$ & 10.5 & 1009.7 & 12.3 & 997.2 & 31.7 & 1015.4 & 10.5 & $\begin{array}{r}101 . \\
8\end{array}$ \\
\hline BU0818-7 & 153 & 13496 & 1.3 & $\begin{array}{r}13.72 \\
99 \\
\end{array}$ & 2.1 & 1.7158 & 2.2 & $\begin{array}{r}0.170 \\
9 \\
\end{array}$ & $\begin{array}{r}0 . \\
5 \\
\end{array}$ & $\begin{array}{r}0.2 \\
3 \\
\end{array}$ & $\begin{array}{r}1016 . \\
8 \\
\end{array}$ & 4.7 & 1014.4 & 13.9 & $\begin{array}{r}1009 . \\
3 \\
\end{array}$ & 42.7 & 1016.8 & 4.7 & $\begin{array}{r}100 . \\
7\end{array}$ \\
\hline $\begin{array}{l}\text { BU0818- } \\
12\end{array}$ & 174 & 15352 & 3.5 & $\begin{array}{r}12.65 \\
93 \\
\end{array}$ & 2.7 & 2.1167 & 2.9 & $\begin{array}{r}0.194 \\
3 \\
\end{array}$ & $\begin{array}{r}1 . \\
1\end{array}$ & $\begin{array}{r}0.3 \\
9 \\
\end{array}$ & $\begin{array}{r}1144 . \\
9 \\
\end{array}$ & 12.0 & 1154.3 & 20.0 & $\begin{array}{r}1172 . \\
0\end{array}$ & 52.7 & 1172.0 & 52.7 & 97.7 \\
\hline $\begin{array}{l}\text { BU0818- } \\
58\end{array}$ & 826 & 90562 & 1.6 & $\begin{array}{r}10.89 \\
99 \\
\end{array}$ & 2.3 & 2.7253 & 2.7 & $\begin{array}{r}0.215 \\
4 \\
\end{array}$ & $\begin{array}{r}1 . \\
4\end{array}$ & $\begin{array}{r}0.5 \\
1 \\
\end{array}$ & $\begin{array}{r}1257 . \\
8 \\
\end{array}$ & 15.9 & 1335.4 & 20.2 & $\begin{array}{r}1462 . \\
1 \\
\end{array}$ & 44.5 & 1462.1 & 44.5 & 86.0 \\
\hline $\begin{array}{l}\text { BU0818- } \\
91\end{array}$ & 558 & 57766 & 2.3 & $\begin{array}{r}10.60 \\
71 \\
\end{array}$ & 1.0 & 3.3485 & 1.5 & $\begin{array}{r}0.257 \\
6 \\
\end{array}$ & $\begin{array}{r}1 . \\
1\end{array}$ & $\begin{array}{r}0.7 \\
6 \\
\end{array}$ & $\begin{array}{r}1477 . \\
5 \\
\end{array}$ & 14.8 & 1492.4 & 11.6 & $\begin{array}{r}1513 . \\
6 \\
\end{array}$ & 18.3 & 1513.6 & 18.3 & 97.6 \\
\hline $\begin{array}{l}\text { BU0818- } \\
64\end{array}$ & 374 & 45230 & 2.3 & $\begin{array}{r}10.22 \\
23\end{array}$ & 1.8 & 3.4760 & 2.0 & $\begin{array}{r}0.257 \\
7\end{array}$ & $\begin{array}{r}0 . \\
9\end{array}$ & $\begin{array}{r}0.4 \\
5\end{array}$ & $\begin{array}{r}1478 . \\
1\end{array}$ & 12.2 & 1521.8 & 16.0 & $\begin{array}{r}1583 . \\
1\end{array}$ & 33.9 & 1583.1 & 33.9 & 93.4 \\
\hline $\begin{array}{l}\text { BU0818- } \\
34\end{array}$ & 285 & 31136 & 2.2 & $\begin{array}{r}10.08 \\
55 \\
\end{array}$ & 1.0 & 3.7738 & 1.2 & $\begin{array}{r}0.276 \\
0 \\
\end{array}$ & $\begin{array}{r}0 . \\
7 \\
\end{array}$ & $\begin{array}{r}0.5 \\
6 \\
\end{array}$ & $\begin{array}{r}1571 . \\
4 \\
\end{array}$ & 9.6 & 1587.2 & 9.9 & $\begin{array}{r}1608 . \\
2 \\
\end{array}$ & 19.1 & 1608.2 & 19.1 & 97.7 \\
\hline $\begin{array}{l}\text { BU0818- } \\
92\end{array}$ & 700 & 43334 & 1.4 & $\begin{array}{r}10.08 \\
33 \\
\end{array}$ & 0.7 & 3.2385 & 0.9 & $\begin{array}{r}0.236 \\
8 \\
\end{array}$ & $\begin{array}{r}0 . \\
5 \\
\end{array}$ & $\begin{array}{r}0.5 \\
9 \\
\end{array}$ & $\begin{array}{r}1370 . \\
2 \\
\end{array}$ & 6.3 & 1466.4 & 6.7 & $\begin{array}{r}1608 . \\
6 \\
\end{array}$ & 13.1 & 1608.6 & 13.1 & 85.2 \\
\hline $\begin{array}{l}\text { BU0818- } \\
39\end{array}$ & 299 & 34156 & 2.6 & $\begin{array}{r}10.08 \\
24 \\
\end{array}$ & 1.3 & 3.5506 & 4.3 & $\begin{array}{r}0.259 \\
6 \\
\end{array}$ & $\begin{array}{r}4 . \\
1 \\
\end{array}$ & $\begin{array}{r}0.9 \\
5 \\
\end{array}$ & $\begin{array}{r}1488 . \\
0 \\
\end{array}$ & 54.7 & 1538.6 & 34.2 & $\begin{array}{r}1608 . \\
8 \\
\end{array}$ & 23.9 & 1608.8 & 23.9 & 92.5 \\
\hline $\begin{array}{l}\text { BU0818- } \\
54\end{array}$ & 307 & 38846 & 0.9 & $\begin{array}{r}10.00 \\
38\end{array}$ & 1.4 & 3.5950 & 1.7 & $\begin{array}{r}0.260 \\
8\end{array}$ & $\begin{array}{r}0 . \\
9\end{array}$ & $\begin{array}{r}0.5 \\
7\end{array}$ & $\begin{array}{r}1494 . \\
1\end{array}$ & 12.5 & 1548.4 & 13.1 & $\begin{array}{r}1623 . \\
4\end{array}$ & 25.3 & 1623.4 & 25.3 & 92.0 \\
\hline $\begin{array}{l}\text { BU0818- } \\
49\end{array}$ & 453 & 24440 & 1.3 & $\begin{array}{r}9.985 \\
7\end{array}$ & 1.7 & 3.6947 & 2.1 & $\begin{array}{r}0.267 \\
6\end{array}$ & $\begin{array}{r}1 . \\
3\end{array}$ & $\begin{array}{r}0.6 \\
0\end{array}$ & $\begin{array}{r}1528 . \\
5\end{array}$ & 17.0 & 1570.2 & 16.8 & $\begin{array}{r}1626 . \\
7\end{array}$ & 31.3 & 1626.7 & 31.3 & 94.0 \\
\hline $\begin{array}{l}\text { BU0818- } \\
110\end{array}$ & 263 & 20202 & 1.1 & $\begin{array}{r}9.969 \\
5\end{array}$ & 2.0 & 3.8374 & 2.1 & $\begin{array}{r}0.277 \\
5\end{array}$ & $\begin{array}{r}0 . \\
5\end{array}$ & $\begin{array}{r}0.2 \\
4\end{array}$ & $\begin{array}{r}1578 . \\
6\end{array}$ & 7.0 & 1600.6 & 16.8 & $\begin{array}{r}1629 . \\
8\end{array}$ & 37.6 & 1629.8 & 37.6 & 96.9 \\
\hline $\begin{array}{l}\text { BU0818- } \\
69\end{array}$ & $\begin{array}{r}135 \\
4 \\
\end{array}$ & $\begin{array}{r}11662 \\
6 \\
\end{array}$ & 4.5 & $\begin{array}{r}9.536 \\
5 \\
\end{array}$ & 2.3 & 3.8230 & 2.8 & $\begin{array}{r}0.264 \\
4 \\
\end{array}$ & $\begin{array}{r}1 . \\
6 \\
\end{array}$ & $\begin{array}{r}0.5 \\
7 \\
\end{array}$ & $\begin{array}{r}1512 . \\
4 \\
\end{array}$ & 21.3 & 1597.6 & 22.2 & $\begin{array}{r}1711 . \\
8 \\
\end{array}$ & 41.6 & 1711.8 & 41.6 & 88.3 \\
\hline $\begin{array}{l}\text { BU0818- } \\
106\end{array}$ & $\begin{array}{r}100 \\
2 \\
\end{array}$ & 61788 & 32.6 & $\begin{array}{r}9.487 \\
5 \\
\end{array}$ & 3.2 & 4.0343 & 3.3 & $\begin{array}{r}0.277 \\
6 \\
\end{array}$ & $\begin{array}{r}0 . \\
8 \\
\end{array}$ & $\begin{array}{r}0.2 \\
5 \\
\end{array}$ & $\begin{array}{r}1579 . \\
3 \\
\end{array}$ & 11.5 & 1641.1 & 26.6 & $\begin{array}{r}1721 . \\
3 \\
\end{array}$ & 58.1 & 1721.3 & 58.1 & 91.7 \\
\hline BU0818- & 381 & 42786 & 1.3 & 9.440 & 2.8 & 4.3631 & 2.9 & 0.298 & 0. & 0.2 & 1685. & 9.9 & 1705.4 & 23.9 & 1730. & 51.6 & 1730.5 & 51.6 & 97.4 \\
\hline
\end{tabular}




\begin{tabular}{|c|c|c|c|c|c|c|c|c|c|c|c|c|c|c|c|c|c|c|c|}
\hline 30 & & & & 4 & & & & 7 & 7 & 3 & 0 & & & & 5 & & & & \\
\hline $\begin{array}{l}\text { BU0818- } \\
36\end{array}$ & 497 & 22572 & 2.7 & $\begin{array}{r}9.386 \\
0\end{array}$ & 1.5 & 3.9560 & 2.5 & $\begin{array}{r}0.269 \\
3\end{array}$ & $\begin{array}{r}2 . \\
1\end{array}$ & $\begin{array}{r}0.8 \\
1\end{array}$ & $\begin{array}{r}1537 . \\
2\end{array}$ & 28.0 & 1625.2 & 20.4 & $\begin{array}{r}1741 . \\
1\end{array}$ & 26.8 & 1741.1 & 26.8 & 88.3 \\
\hline $\begin{array}{l}\text { BU0818- } \\
101\end{array}$ & $\begin{array}{r}140 \\
6\end{array}$ & 24342 & 2.3 & $\begin{array}{r}9.104 \\
7\end{array}$ & 1.7 & 3.9614 & 2.4 & $\begin{array}{r}0.261 \\
6\end{array}$ & $\begin{array}{l}1 . \\
7\end{array}$ & $\begin{array}{r}0.7 \\
2\end{array}$ & $\begin{array}{r}1498 . \\
0\end{array}$ & 23.0 & 1626.3 & 19.3 & $\begin{array}{r}1796 . \\
6\end{array}$ & 30.1 & 1796.6 & 30.1 & 83.4 \\
\hline $\begin{array}{l}\text { BU0818- } \\
78\end{array}$ & 545 & 68038 & 7.7 & $\begin{array}{r}9.093 \\
0\end{array}$ & 1.0 & 4.8601 & 2.2 & $\begin{array}{r}0.320 \\
5\end{array}$ & $\begin{array}{r}2 . \\
0\end{array}$ & $\begin{array}{r}0.9 \\
0\end{array}$ & $\begin{array}{r}1792 . \\
3\end{array}$ & 31.6 & 1795.4 & 18.9 & $\begin{array}{r}1799 . \\
0\end{array}$ & 17.8 & 1799.0 & 17.8 & 99.6 \\
\hline $\begin{array}{l}\text { BU0818- } \\
41\end{array}$ & 303 & 39690 & 3.3 & $\begin{array}{r}9.035 \\
5 \\
\end{array}$ & 2.0 & 4.6019 & 2.5 & $\begin{array}{r}0.301 \\
6 \\
\end{array}$ & $\begin{array}{r}1 . \\
5\end{array}$ & $\begin{array}{r}0.5 \\
8\end{array}$ & $\begin{array}{r}1699 . \\
1\end{array}$ & 21.7 & 1749.6 & 20.7 & $\begin{array}{r}1810 . \\
5\end{array}$ & 36.7 & 1810.5 & 36.7 & 93.8 \\
\hline $\begin{array}{l}\text { BU0818- } \\
42\end{array}$ & 339 & 27890 & 8.8 & $\begin{array}{r}8.839 \\
2 \\
\end{array}$ & 1.1 & 4.0847 & 2.2 & $\begin{array}{r}0.261 \\
9 \\
\end{array}$ & $\begin{array}{r}1 . \\
9\end{array}$ & $\begin{array}{r}0.8 \\
6 \\
\end{array}$ & $\begin{array}{r}1499 . \\
4\end{array}$ & 25.3 & 1651.3 & 18.0 & $\begin{array}{r}1850 . \\
3 \\
\end{array}$ & 20.6 & 1850.3 & 20.6 & 81.0 \\
\hline $\begin{array}{l}\text { BU0818- } \\
56\end{array}$ & 402 & 34970 & 4.9 & $\begin{array}{r}8.833 \\
7\end{array}$ & 2.1 & 4.5226 & 2.5 & $\begin{array}{r}0.289 \\
8\end{array}$ & $\begin{array}{r}1 . \\
4\end{array}$ & $\begin{array}{r}0.5 \\
4\end{array}$ & $\begin{array}{r}1640 . \\
3\end{array}$ & 19.8 & 1735.1 & 21.0 & $\begin{array}{r}1851 . \\
4\end{array}$ & 38.3 & 1851.4 & 38.3 & 88.6 \\
\hline $\begin{array}{l}\text { BU0818- } \\
109\end{array}$ & 427 & 23678 & 2.5 & $\begin{array}{r}8.795 \\
1\end{array}$ & 1.5 & 4.0644 & 2.4 & $\begin{array}{r}0.259 \\
3\end{array}$ & $\begin{array}{r}1 . \\
9\end{array}$ & $\begin{array}{r}0.7 \\
8\end{array}$ & $\begin{array}{r}1486 . \\
0\end{array}$ & 25.5 & 1647.2 & 20.0 & $\begin{array}{r}1859 . \\
3\end{array}$ & 27.5 & 1859.3 & 27.5 & 79.9 \\
\hline $\begin{array}{l}\text { BU0818- } \\
31\end{array}$ & 170 & 20786 & 2.7 & $\begin{array}{r}8.793 \\
1 \\
\end{array}$ & 1.9 & 4.7897 & 2.9 & $\begin{array}{r}0.305 \\
5 \\
\end{array}$ & $\begin{array}{r}2 . \\
2\end{array}$ & $\begin{array}{r}0.7 \\
6 \\
\end{array}$ & $\begin{array}{r}1718 . \\
3\end{array}$ & 32.9 & 1783.1 & 24.0 & $\begin{array}{r}1859 . \\
8 \\
\end{array}$ & 33.4 & 1859.8 & 33.4 & 92.4 \\
\hline $\begin{array}{l}\text { BU0818- } \\
102\end{array}$ & 260 & 28894 & 2.0 & $\begin{array}{r}8.769 \\
1 \\
\end{array}$ & 1.9 & 5.2556 & 1.9 & $\begin{array}{r}0.334 \\
3 \\
\end{array}$ & $\begin{array}{r}0 . \\
5\end{array}$ & $\begin{array}{r}0.2 \\
6 \\
\end{array}$ & $\begin{array}{r}1859 . \\
0 \\
\end{array}$ & 8.1 & 1861.7 & 16.4 & $\begin{array}{r}1864 . \\
7 \\
\end{array}$ & 33.6 & 1864.7 & 33.6 & 99.7 \\
\hline $\begin{array}{l}\text { BU0818- } \\
75\end{array}$ & 97 & 8074 & 1.8 & $\begin{array}{r}8.765 \\
6 \\
\end{array}$ & 2.2 & 4.5124 & 2.4 & $\begin{array}{r}0.286 \\
9 \\
\end{array}$ & $\begin{array}{r}1 . \\
2\end{array}$ & $\begin{array}{r}0.4 \\
7 \\
\end{array}$ & $\begin{array}{r}1625 . \\
9\end{array}$ & 16.5 & 1733.3 & 20.3 & $\begin{array}{r}1865 . \\
4\end{array}$ & 38.9 & 1865.4 & 38.9 & 87.2 \\
\hline $\begin{array}{l}\text { BU0818- } \\
27\end{array}$ & 157 & 23786 & 2.7 & $\begin{array}{r}8.714 \\
0\end{array}$ & 2.0 & 4.9632 & 2.4 & $\begin{array}{r}0.313 \\
7\end{array}$ & $\begin{array}{l}1 . \\
4\end{array}$ & $\begin{array}{r}0.5 \\
8\end{array}$ & $\begin{array}{r}1758 . \\
8\end{array}$ & 21.7 & 1813.1 & 20.7 & $\begin{array}{r}1876 . \\
1\end{array}$ & 36.1 & 1876.1 & 36.1 & 93.7 \\
\hline $\begin{array}{l}\text { BU0818- } \\
52\end{array}$ & 521 & 49636 & 4.6 & $\begin{array}{r}8.706 \\
7\end{array}$ & 1.3 & 4.9730 & 1.7 & $\begin{array}{r}0.314 \\
0\end{array}$ & $\begin{array}{r}1 . \\
2\end{array}$ & $\begin{array}{r}0.6 \\
7\end{array}$ & $\begin{array}{r}1760 . \\
5\end{array}$ & 17.9 & 1814.7 & 14.7 & $\begin{array}{r}1877 . \\
6\end{array}$ & 23.3 & 1877.6 & 23.3 & 93.8 \\
\hline $\begin{array}{l}\text { BU0818- } \\
93\end{array}$ & $\begin{array}{r}125 \\
7\end{array}$ & 68124 & 10.6 & $\begin{array}{r}8.706 \\
3\end{array}$ & 1.7 & 4.3875 & 2.0 & $\begin{array}{r}0.277 \\
0\end{array}$ & $\begin{array}{r}1 . \\
1\end{array}$ & $\begin{array}{r}0.5 \\
4\end{array}$ & $\begin{array}{r}1576 . \\
5\end{array}$ & 15.2 & 1710.0 & 16.6 & $\begin{array}{r}1877 . \\
7\end{array}$ & 30.3 & 1877.7 & 30.3 & 84.0 \\
\hline $\begin{array}{l}\text { BU0818- } \\
45\end{array}$ & 740 & 97120 & 16.9 & $\begin{array}{r}8.601 \\
3 \\
\end{array}$ & 1.1 & 5.4482 & 1.4 & $\begin{array}{r}0.339 \\
9 \\
\end{array}$ & $\begin{array}{r}0 . \\
9\end{array}$ & $\begin{array}{r}0.6 \\
4 \\
\end{array}$ & $\begin{array}{r}1886 . \\
1\end{array}$ & 14.9 & 1892.5 & 12.2 & $\begin{array}{r}1899 . \\
5 \\
\end{array}$ & 19.6 & 1899.5 & 19.6 & 99.3 \\
\hline $\begin{array}{l}\text { BU0818- } \\
65\end{array}$ & 198 & 37852 & 2.5 & $\begin{array}{r}8.597 \\
0\end{array}$ & 1.9 & 4.9679 & 2.8 & $\begin{array}{r}0.309 \\
8\end{array}$ & $\begin{array}{r}2 . \\
0\end{array}$ & $\begin{array}{r}0.7 \\
3\end{array}$ & $\begin{array}{r}1739 . \\
5\end{array}$ & 30.6 & 1813.9 & 23.4 & $\begin{array}{r}1900 . \\
4\end{array}$ & 34.2 & 1900.4 & 34.2 & 91.5 \\
\hline $\begin{array}{l}\text { BU0818- } \\
53\end{array}$ & 659 & 83892 & 6.9 & $\begin{array}{r}8.485 \\
8\end{array}$ & 1.5 & 5.4820 & 1.7 & $\begin{array}{r}0.337 \\
4\end{array}$ & $\begin{array}{l}0 . \\
7\end{array}$ & $\begin{array}{r}0.4 \\
1\end{array}$ & $\begin{array}{r}1874 . \\
1\end{array}$ & 11.2 & 1897.8 & 14.5 & $\begin{array}{r}1923 . \\
8\end{array}$ & 27.6 & 1923.8 & 27.6 & 97.4 \\
\hline $\begin{array}{l}\text { BU0818- } \\
83\end{array}$ & 308 & 1226 & 1.7 & $\begin{array}{r}8.476 \\
8\end{array}$ & 8.3 & 4.4005 & 8.6 & $\begin{array}{r}0.270 \\
5\end{array}$ & $\begin{array}{r}2 . \\
4\end{array}$ & $\begin{array}{r}0.2 \\
8\end{array}$ & $\begin{array}{r}1543 . \\
5\end{array}$ & 33.2 & 1712.4 & 71.6 & $\begin{array}{r}1925 . \\
7\end{array}$ & $\begin{array}{r}148 . \\
8\end{array}$ & 1925.7 & $\begin{array}{r}148 . \\
8\end{array}$ & 80.2 \\
\hline $\begin{array}{l}\text { BU0818- } \\
16\end{array}$ & 467 & 61836 & 7.7 & $\begin{array}{r}8.356 \\
2\end{array}$ & 1.3 & 5.2024 & 1.8 & $\begin{array}{r}0.315 \\
3\end{array}$ & $\begin{array}{r}1 . \\
2\end{array}$ & $\begin{array}{r}0.6 \\
8\end{array}$ & $\begin{array}{r}1766 . \\
7\end{array}$ & 18.4 & 1853.0 & 15.0 & $\begin{array}{r}1951 . \\
3\end{array}$ & 23.2 & 1951.3 & 23.2 & 90.5 \\
\hline $\begin{array}{l}\text { BU0818- } \\
50\end{array}$ & 136 & 26484 & 1.3 & $\begin{array}{r}7.988 \\
5 \\
\end{array}$ & 1.4 & 5.8617 & 1.8 & $\begin{array}{r}0.339 \\
6 \\
\end{array}$ & $\begin{array}{r}1 . \\
1\end{array}$ & $\begin{array}{r}0.6 \\
2 \\
\end{array}$ & $\begin{array}{r}1884 . \\
8\end{array}$ & 18.6 & 1955.6 & 15.9 & $\begin{array}{r}2031 . \\
3\end{array}$ & 25.5 & 2031.3 & 25.5 & 92.8 \\
\hline BU0818-6 & 146 & 27562 & 1.3 & $\begin{array}{r}7.826 \\
1\end{array}$ & 3.6 & 6.4529 & 3.7 & $\begin{array}{r}0.366 \\
3\end{array}$ & $\begin{array}{r}0 . \\
8\end{array}$ & $\begin{array}{r}0.2 \\
2\end{array}$ & $\begin{array}{r}2011 . \\
8\end{array}$ & 13.7 & 2039.5 & 32.2 & $\begin{array}{r}2067 . \\
6\end{array}$ & 63.1 & 2067.6 & 63.1 & 97.3 \\
\hline $\begin{array}{l}\text { BU0818- } \\
59\end{array}$ & 287 & 46434 & 1.8 & $\begin{array}{r}7.800 \\
3\end{array}$ & 2.0 & 6.2827 & 2.2 & $\begin{array}{r}0.355 \\
4\end{array}$ & $\begin{array}{l}1 . \\
0\end{array}$ & $\begin{array}{r}0.4 \\
3\end{array}$ & $\begin{array}{r}1960 . \\
5\end{array}$ & 16.1 & 2016.0 & 19.4 & $\begin{array}{r}2073 . \\
4\end{array}$ & 35.2 & 2073.4 & 35.2 & 94.6 \\
\hline $\begin{array}{l}\text { BU0818- } \\
35\end{array}$ & $\begin{array}{r}102 \\
3\end{array}$ & $\begin{array}{r}12436 \\
0\end{array}$ & 4.0 & $\begin{array}{r}7.699 \\
9\end{array}$ & 1.2 & 6.4825 & 1.3 & $\begin{array}{r}0.362 \\
0\end{array}$ & $\begin{array}{r}0 . \\
5\end{array}$ & $\begin{array}{r}0.3 \\
9\end{array}$ & $\begin{array}{r}1991 . \\
7\end{array}$ & 8.7 & 2043.5 & 11.6 & $\begin{array}{r}2096 . \\
2\end{array}$ & 21.3 & 2096.2 & 21.3 & 95.0 \\
\hline $\begin{array}{l}\text { BU0818- } \\
76\end{array}$ & $\begin{array}{r}101 \\
1\end{array}$ & $\begin{array}{r}13906 \\
6\end{array}$ & 5.7 & $\begin{array}{r}7.648 \\
9\end{array}$ & 1.8 & 6.4778 & 2.0 & $\begin{array}{r}0.359 \\
4\end{array}$ & $\begin{array}{c}0 . \\
7\end{array}$ & $\begin{array}{r}0.3 \\
6\end{array}$ & $\begin{array}{r}1979 . \\
1\end{array}$ & 11.9 & 2042.9 & 17.3 & $\begin{array}{r}2107 . \\
9\end{array}$ & 32.3 & 2107.9 & 32.3 & 93.9 \\
\hline $\begin{array}{l}\text { BU0818- } \\
80\end{array}$ & 76 & 16762 & 1.2 & $\begin{array}{r}7.600 \\
7\end{array}$ & 1.6 & 7.1550 & 1.8 & $\begin{array}{r}0.394 \\
4\end{array}$ & $\begin{array}{r}0 . \\
8\end{array}$ & $\begin{array}{r}0.4 \\
5\end{array}$ & $\begin{array}{r}2143 . \\
3\end{array}$ & 15.1 & 2130.9 & 16.4 & $\begin{array}{r}2119 . \\
0\end{array}$ & 28.8 & 2119.0 & 28.8 & $\begin{array}{r}101 . \\
1\end{array}$ \\
\hline
\end{tabular}




\begin{tabular}{|c|c|c|c|c|c|c|c|c|c|c|c|c|c|c|c|c|c|c|c|}
\hline $\begin{array}{l}\text { BU0818- } \\
82\end{array}$ & 442 & 56886 & 1.9 & $\begin{array}{r}7.563 \\
3 \\
\end{array}$ & 1.1 & 7.1566 & 1.2 & $\begin{array}{r}0.392 \\
6 \\
\end{array}$ & $\begin{array}{r}0 . \\
5\end{array}$ & $\begin{array}{r}0.4 \\
1 \\
\end{array}$ & $\begin{array}{r}2134 . \\
7 \\
\end{array}$ & 9.1 & 2131.1 & 10.8 & $\begin{array}{r}2127 . \\
6 \\
\end{array}$ & 19.4 & 2127.6 & 19.4 & $\begin{array}{r}100 . \\
3 \\
\end{array}$ \\
\hline $\begin{array}{l}\text { BU0818- } \\
10\end{array}$ & 223 & 38536 & 2.0 & $\begin{array}{r}7.092 \\
0\end{array}$ & 1.0 & 7.5445 & 2.1 & $\begin{array}{r}0.388 \\
1\end{array}$ & $\begin{array}{r}1 . \\
8\end{array}$ & $\begin{array}{r}0.8 \\
7\end{array}$ & $\begin{array}{r}2113 . \\
8\end{array}$ & 32.4 & 2178.3 & 18.5 & $\begin{array}{r}2239 . \\
6\end{array}$ & 17.5 & 2239.6 & 17.5 & 94.4 \\
\hline $\begin{array}{l}\text { BU0818- } \\
97\end{array}$ & 320 & 21504 & 1.9 & $\begin{array}{r}6.455 \\
1\end{array}$ & 1.4 & 6.9177 & 2.9 & $\begin{array}{r}0.323 \\
9\end{array}$ & $\begin{array}{r}2 . \\
6\end{array}$ & $\begin{array}{r}0.8 \\
9\end{array}$ & $\begin{array}{r}1808 . \\
6\end{array}$ & 40.7 & 2100.9 & 25.8 & $\begin{array}{r}2400 . \\
9\end{array}$ & 23.0 & 2400.9 & 23.0 & 75.3 \\
\hline $\begin{array}{l}\text { BU0818- } \\
22\end{array}$ & 245 & 26126 & 3.5 & $\begin{array}{r}6.436 \\
5 \\
\end{array}$ & 1.7 & 6.5125 & 2.8 & $\begin{array}{r}0.304 \\
0 \\
\end{array}$ & $\begin{array}{r}2 . \\
2\end{array}$ & $\begin{array}{r}0.8 \\
0 \\
\end{array}$ & $\begin{array}{r}1711 . \\
2\end{array}$ & 33.4 & 2047.6 & 24.5 & $\begin{array}{r}2405 . \\
8\end{array}$ & 28.4 & 2405.8 & 28.4 & 71.1 \\
\hline $\begin{array}{l}\text { BU0818- } \\
63\end{array}$ & $\begin{array}{r}112 \\
9 \\
\end{array}$ & $\begin{array}{r}12088 \\
0 \\
\end{array}$ & 5.1 & $\begin{array}{r}6.326 \\
6 \\
\end{array}$ & 3.3 & 8.3937 & 3.9 & $\begin{array}{r}0.385 \\
1 \\
\end{array}$ & $\begin{array}{r}2 . \\
0\end{array}$ & $\begin{array}{r}0.5 \\
3 \\
\end{array}$ & $\begin{array}{r}2100 . \\
2 \\
\end{array}$ & 36.4 & 2274.5 & 35.1 & $\begin{array}{r}2435 . \\
0 \\
\end{array}$ & 55.8 & 2435.0 & 55.8 & 86.3 \\
\hline $\begin{array}{l}\text { BU0818- } \\
79\end{array}$ & 81 & 14216 & 1.2 & $\begin{array}{r}6.304 \\
0 \\
\end{array}$ & 1.7 & 9.0678 & 2.1 & $\begin{array}{r}0.414 \\
6 \\
\end{array}$ & $\begin{array}{r}1 . \\
3\end{array}$ & $\begin{array}{r}0.6 \\
2 \\
\end{array}$ & $\begin{array}{r}2235 . \\
9 \\
\end{array}$ & 24.9 & 2344.9 & 19.3 & $\begin{array}{r}2441 . \\
1\end{array}$ & 28.0 & 2441.1 & 28.0 & 91.6 \\
\hline $\begin{array}{l}\text { BU0818- } \\
28\end{array}$ & 133 & 13044 & 2.1 & $\begin{array}{r}6.250 \\
5\end{array}$ & 1.3 & 7.1836 & 3.8 & $\begin{array}{r}0.325 \\
7\end{array}$ & $\begin{array}{r}3 . \\
6\end{array}$ & $\begin{array}{r}0.9 \\
4\end{array}$ & $\begin{array}{r}1817 . \\
3\end{array}$ & 56.9 & 2134.5 & 34.2 & $\begin{array}{r}2455 . \\
5\end{array}$ & 22.6 & 2455.5 & 22.6 & 74.0 \\
\hline $\begin{array}{l}\text { BU0818- } \\
71\end{array}$ & 169 & 44102 & 1.8 & $\begin{array}{r}6.173 \\
6 \\
\end{array}$ & 1.0 & $\begin{array}{r}10.460 \\
4\end{array}$ & 1.3 & $\begin{array}{r}0.468 \\
4\end{array}$ & $\begin{array}{r}0 . \\
9\end{array}$ & $\begin{array}{r}0.6 \\
7\end{array}$ & $\begin{array}{r}2476 . \\
4\end{array}$ & 18.3 & 2476.4 & 12.3 & $\begin{array}{r}2476 . \\
4\end{array}$ & 16.5 & 2476.4 & 16.5 & $\begin{array}{r}100 . \\
0\end{array}$ \\
\hline $\begin{array}{l}\text { BU0818- } \\
62\end{array}$ & 76 & 16828 & 1.7 & $\begin{array}{r}6.166 \\
3 \\
\end{array}$ & 0.6 & $\begin{array}{r}10.039 \\
0 \\
\end{array}$ & 1.2 & $\begin{array}{r}0.449 \\
0 \\
\end{array}$ & $\begin{array}{r}1 . \\
1\end{array}$ & $\begin{array}{r}0.8 \\
8\end{array}$ & $\begin{array}{r}2390 . \\
7\end{array}$ & 21.6 & 2438.4 & 11.4 & $\begin{array}{r}2478 . \\
4\end{array}$ & 10.0 & 2478.4 & 10.0 & 96.5 \\
\hline $\begin{array}{l}\text { BU0818- } \\
43\end{array}$ & 427 & 63204 & 2.0 & $\begin{array}{r}6.159 \\
3 \\
\end{array}$ & 1.2 & 9.6386 & 1.6 & $\begin{array}{r}0.430 \\
6 \\
\end{array}$ & $\begin{array}{r}0 . \\
9\end{array}$ & $\begin{array}{r}0.6 \\
0 \\
\end{array}$ & $\begin{array}{r}2308 . \\
3 \\
\end{array}$ & 18.2 & 2400.9 & 14.3 & $\begin{array}{r}2480 . \\
3 \\
\end{array}$ & 20.9 & 2480.3 & 20.9 & 93.1 \\
\hline $\begin{array}{l}\text { BU0818- } \\
19\end{array}$ & 226 & 20958 & 4.6 & $\begin{array}{r}6.130 \\
1\end{array}$ & 0.9 & 8.0111 & 1.4 & $\begin{array}{r}0.356 \\
2\end{array}$ & $\begin{array}{r}1 . \\
1\end{array}$ & $\begin{array}{r}0.7 \\
5\end{array}$ & $\begin{array}{r}1964 . \\
0\end{array}$ & 17.8 & 2232.3 & 12.7 & $\begin{array}{r}2488 . \\
3\end{array}$ & 15.7 & 2488.3 & 15.7 & 78.9 \\
\hline $\begin{array}{l}\text { BU0818- } \\
46\end{array}$ & 444 & 63646 & 2.2 & $\begin{array}{r}6.127 \\
7\end{array}$ & 1.2 & $\begin{array}{r}10.337 \\
0 \\
\end{array}$ & 1.5 & $\begin{array}{r}0.459 \\
4\end{array}$ & $\begin{array}{r}0 . \\
9\end{array}$ & $\begin{array}{r}0.6 \\
0\end{array}$ & $\begin{array}{r}2436 . \\
9\end{array}$ & 17.9 & 2465.4 & 13.6 & $\begin{array}{r}2489 . \\
0\end{array}$ & 19.7 & 2489.0 & 19.7 & 97.9 \\
\hline $\begin{array}{l}\text { BU0818- } \\
99\end{array}$ & 265 & 48564 & 1.7 & $\begin{array}{r}6.121 \\
6 \\
\end{array}$ & 1.3 & $\begin{array}{r}10.691 \\
5 \\
\end{array}$ & 1.5 & $\begin{array}{r}0.474 \\
7 \\
\end{array}$ & $\begin{array}{r}0 . \\
7\end{array}$ & $\begin{array}{r}0.4 \\
8 \\
\end{array}$ & $\begin{array}{r}2504 . \\
1 \\
\end{array}$ & 14.9 & 2496.7 & 14.0 & $\begin{array}{r}2490 . \\
7\end{array}$ & 22.2 & 2490.7 & 22.2 & $\begin{array}{r}100 . \\
5 \\
\end{array}$ \\
\hline $\begin{array}{l}\text { BU0818- } \\
51\end{array}$ & 519 & 64712 & 3.5 & $\begin{array}{r}6.099 \\
9 \\
\end{array}$ & 1.0 & 9.4084 & 3.5 & $\begin{array}{r}0.416 \\
2 \\
\end{array}$ & $\begin{array}{r}3 . \\
4 \\
\end{array}$ & $\begin{array}{r}0.9 \\
6 \\
\end{array}$ & $\begin{array}{r}2243 . \\
4 \\
\end{array}$ & 63.5 & 2378.6 & 32.0 & $\begin{array}{r}2496 . \\
7 \\
\end{array}$ & 16.5 & 2496.7 & 16.5 & 89.9 \\
\hline $\begin{array}{l}\text { BU0818- } \\
84\end{array}$ & 678 & $\begin{array}{r}11494 \\
6 \\
\end{array}$ & 1.9 & $\begin{array}{r}6.094 \\
7 \\
\end{array}$ & 2.0 & $\begin{array}{r}10.180 \\
3 \\
\end{array}$ & 2.3 & $\begin{array}{r}0.450 \\
0 \\
\end{array}$ & $\begin{array}{r}1 . \\
1\end{array}$ & $\begin{array}{r}0.4 \\
7 \\
\end{array}$ & $\begin{array}{r}2395 . \\
3 \\
\end{array}$ & 21.8 & 2451.3 & 21.2 & $\begin{array}{r}2498 . \\
1 \\
\end{array}$ & 34.0 & 2498.1 & 34.0 & 95.9 \\
\hline $\begin{array}{l}\text { BU0818- } \\
40\end{array}$ & 181 & 23468 & 1.4 & $\begin{array}{r}6.085 \\
1\end{array}$ & 1.2 & 9.7343 & 2.1 & $\begin{array}{r}0.429 \\
6\end{array}$ & $\begin{array}{r}1 . \\
7\end{array}$ & $\begin{array}{r}0.8 \\
1\end{array}$ & $\begin{array}{r}2303 . \\
9\end{array}$ & 33.3 & 2410.0 & 19.5 & $\begin{array}{r}2500 . \\
8\end{array}$ & 20.9 & 2500.8 & 20.9 & 92.1 \\
\hline $\begin{array}{l}\text { BU0818- } \\
11\end{array}$ & 102 & 23950 & 1.7 & $\begin{array}{r}6.037 \\
1 \\
\end{array}$ & 2.0 & $\begin{array}{r}10.799 \\
6 \\
\end{array}$ & 2.3 & $\begin{array}{r}0.472 \\
9 \\
\end{array}$ & $\begin{array}{r}1 . \\
2\end{array}$ & $\begin{array}{r}0.5 \\
1 \\
\end{array}$ & $\begin{array}{r}2496 . \\
1 \\
\end{array}$ & 24.8 & 2506.0 & 21.7 & $\begin{array}{r}2514 . \\
1 \\
\end{array}$ & 33.6 & 2514.1 & 33.6 & 99.3 \\
\hline $\begin{array}{l}\text { BU0818- } \\
55\end{array}$ & 468 & 51730 & 6.4 & $\begin{array}{r}6.008 \\
8\end{array}$ & 1.2 & 7.9203 & 2.8 & $\begin{array}{r}0.345 \\
2\end{array}$ & $\begin{array}{r}2 . \\
5\end{array}$ & $\begin{array}{r}0.9 \\
0\end{array}$ & $\begin{array}{r}1911 . \\
5\end{array}$ & 41.5 & 2222.0 & 25.2 & $\begin{array}{r}2522 . \\
0\end{array}$ & 20.8 & 2522.0 & 20.8 & 75.8 \\
\hline $\begin{array}{l}\text { BU0818- } \\
37\end{array}$ & 205 & 46458 & 1.8 & $\begin{array}{r}5.995 \\
9 \\
\end{array}$ & 1.8 & $\begin{array}{r}10.716 \\
6 \\
\end{array}$ & 2.4 & $\begin{array}{r}0.466 \\
0 \\
\end{array}$ & $\begin{array}{r}1 . \\
6 \\
\end{array}$ & $\begin{array}{r}0.6 \\
7 \\
\end{array}$ & $\begin{array}{r}2466 . \\
1 \\
\end{array}$ & 33.4 & 2498.9 & 22.7 & $\begin{array}{r}2525 . \\
6 \\
\end{array}$ & 30.6 & 2525.6 & 30.6 & 97.6 \\
\hline $\begin{array}{l}\text { BU0818- } \\
100\end{array}$ & 755 & $\begin{array}{r}10853 \\
2\end{array}$ & 2.6 & $\begin{array}{r}5.980 \\
8\end{array}$ & 0.5 & $\begin{array}{r}10.844 \\
3\end{array}$ & 0.7 & $\begin{array}{r}0.470 \\
4\end{array}$ & $\begin{array}{l}0 . \\
5\end{array}$ & $\begin{array}{r}0.6 \\
9\end{array}$ & $\begin{array}{r}2485 . \\
3\end{array}$ & 10.3 & 2509.9 & 6.8 & $\begin{array}{r}2529 . \\
8\end{array}$ & 8.9 & 2529.8 & 8.9 & 98.2 \\
\hline BU0818-9 & 143 & 20690 & 1.4 & $\begin{array}{r}5.959 \\
9\end{array}$ & 1.4 & $\begin{array}{r}10.874 \\
8\end{array}$ & 1.6 & $\begin{array}{r}0.470 \\
1\end{array}$ & $\begin{array}{l}0 . \\
7\end{array}$ & $\begin{array}{r}0.4 \\
4\end{array}$ & $\begin{array}{r}2483 . \\
8\end{array}$ & 14.4 & 2512.5 & 14.6 & $\begin{array}{r}2535 . \\
7\end{array}$ & 23.7 & 2535.7 & 23.7 & 98.0 \\
\hline $\begin{array}{l}\text { BU0818- } \\
14\end{array}$ & 99 & 30822 & 2.5 & $\begin{array}{r}5.956 \\
6\end{array}$ & 3.1 & $\begin{array}{r}10.371 \\
7\end{array}$ & 3.3 & $\begin{array}{r}0.448 \\
1\end{array}$ & $\begin{array}{r}1 . \\
1\end{array}$ & $\begin{array}{r}0.3 \\
4\end{array}$ & $\begin{array}{r}2386 . \\
7\end{array}$ & 22.1 & 2468.5 & 30.2 & $\begin{array}{r}2536 . \\
6\end{array}$ & 51.3 & 2536.6 & 51.3 & 94.1 \\
\hline $\begin{array}{l}\text { BU0818- } \\
57\end{array}$ & 345 & 61934 & 3.5 & $\begin{array}{r}5.948 \\
4 \\
\end{array}$ & 2.4 & $\begin{array}{r}10.004 \\
6 \\
\end{array}$ & 2.7 & $\begin{array}{r}0.431 \\
6 \\
\end{array}$ & $\begin{array}{r}1 . \\
2\end{array}$ & $\begin{array}{r}0.4 \\
5 \\
\end{array}$ & $\begin{array}{r}2313 . \\
0 \\
\end{array}$ & 23.3 & 2435.2 & 24.7 & $\begin{array}{r}2538 . \\
9 \\
\end{array}$ & 40.1 & 2538.9 & 40.1 & 91.1 \\
\hline $\begin{array}{l}\text { BU0818- } \\
95\end{array}$ & 390 & 69842 & 1.1 & $\begin{array}{r}5.918 \\
8 \\
\end{array}$ & 0.7 & $\begin{array}{r}11.079 \\
3 \\
\end{array}$ & 1.3 & $\begin{array}{r}0.475 \\
6 \\
\end{array}$ & $\begin{array}{r}1 . \\
1\end{array}$ & $\begin{array}{r}0.8 \\
6 \\
\end{array}$ & $\begin{array}{r}2508 . \\
1 \\
\end{array}$ & 23.1 & 2529.8 & 12.0 & $\begin{array}{r}2547 . \\
3 \\
\end{array}$ & 11.1 & 2547.3 & 11.1 & 98.5 \\
\hline BU0818- & 530 & 67396 & 1.7 & 5.516 & 1.7 & 10.874 & 2.7 & 0.435 & 2. & 0.7 & 2328. & 40.1 & 2512.4 & 24.9 & 2664. & 28.5 & 2664.5 & 28.5 & 87.4 \\
\hline
\end{tabular}




\begin{tabular}{|c|c|c|c|c|c|c|c|c|c|c|c|c|c|c|c|c|c|c|c|}
\hline 68 & & & & 8 & & 3 & & 1 & 1 & 7 & 7 & & & & 5 & & & & \\
\hline $\begin{array}{l}\text { BU0818- } \\
13\end{array}$ & 572 & 44160 & 4.1 & $\begin{array}{r}5.499 \\
6\end{array}$ & 2.8 & $\begin{array}{r}10.807 \\
0\end{array}$ & 3.8 & $\begin{array}{r}0.431 \\
1\end{array}$ & $\begin{array}{r}2 . \\
6\end{array}$ & $\begin{array}{r}0.6 \\
8\end{array}$ & $\begin{array}{r}2310 . \\
5\end{array}$ & 50.1 & 2506.7 & 35.3 & $\begin{array}{r}2669 . \\
6\end{array}$ & 46.0 & 2669.6 & 46.0 & 86.5 \\
\hline $\begin{array}{l}\text { BU0818- } \\
24\end{array}$ & 275 & 75888 & 5.2 & $\begin{array}{r}4.655 \\
4\end{array}$ & 1.2 & $\begin{array}{r}15.459 \\
5\end{array}$ & 1.6 & $\begin{array}{r}0.522 \\
0\end{array}$ & $\begin{array}{r}1 . \\
1\end{array}$ & $\begin{array}{r}0.6 \\
9\end{array}$ & $\begin{array}{r}2707 . \\
6\end{array}$ & 24.3 & 2844.0 & 15.2 & $\begin{array}{r}2942 . \\
1\end{array}$ & 18.7 & 2942.1 & 18.7 & 92.0 \\
\hline $\begin{array}{l}\text { BU0818- } \\
74\end{array}$ & 218 & 18626 & 2.7 & $\begin{array}{r}3.817 \\
2\end{array}$ & 2.6 & $\begin{array}{r}16.812 \\
0\end{array}$ & 2.8 & $\begin{array}{r}0.465 \\
4\end{array}$ & $\begin{array}{r}1 . \\
2\end{array}$ & $\begin{array}{r}0.4 \\
2\end{array}$ & $\begin{array}{r}2463 . \\
5\end{array}$ & 24.2 & 2924.2 & 27.1 & $\begin{array}{r}3258 . \\
6\end{array}$ & 40.5 & 3258.6 & 40.5 & 75.6 \\
\hline $\begin{array}{l}\text { BU0818- } \\
85\end{array}$ & 302 & 32558 & 1.4 & $\begin{array}{r}3.354 \\
5\end{array}$ & 1.4 & $\begin{array}{r}21.590 \\
2\end{array}$ & 2.3 & $\begin{array}{r}0.525 \\
3\end{array}$ & $\begin{array}{r}1 . \\
9\end{array}$ & $\begin{array}{r}0.7 \\
9\end{array}$ & $\begin{array}{r}2721 . \\
5\end{array}$ & 41.1 & 3165.5 & 22.7 & $\begin{array}{r}3460 . \\
4\end{array}$ & 22.2 & 3460.4 & 22.2 & 78.6 \\
\hline $\begin{array}{l}\text { BU0818- } \\
66\end{array}$ & $\begin{array}{r}125 \\
1 \\
\end{array}$ & $\begin{array}{r}26442 \\
0 \\
\end{array}$ & 4.8 & $\begin{array}{r}3.316 \\
3 \\
\end{array}$ & 2.4 & $\begin{array}{r}25.421 \\
0 \\
\end{array}$ & 3.8 & $\begin{array}{r}0.611 \\
4 \\
\end{array}$ & $\begin{array}{r}3 . \\
0 \\
\end{array}$ & $\begin{array}{r}0.7 \\
8 \\
\end{array}$ & $\begin{array}{r}3075 . \\
7 \\
\end{array}$ & 73.4 & 3324.5 & 37.6 & $\begin{array}{r}3478 . \\
1 \\
\end{array}$ & 37.2 & 3478.1 & 37.2 & 88.4 \\
\hline $\begin{array}{l}\text { BU0872- } \\
21\end{array}$ & 267 & 10440 & 2.1 & $\begin{array}{r}17.49 \\
23\end{array}$ & 4.7 & 0.6623 & 4.9 & $\begin{array}{r}0.084 \\
0\end{array}$ & $\begin{array}{l}1 . \\
3\end{array}$ & $\begin{array}{r}0.2 \\
6\end{array}$ & 520.1 & 6.3 & 516.0 & 19.8 & 498.0 & $\begin{array}{r}104 . \\
1\end{array}$ & 520.1 & 6.3 & $\begin{array}{r}104 . \\
4\end{array}$ \\
\hline BU0872-4 & 636 & 26188 & 1.7 & $\begin{array}{r}17.18 \\
73\end{array}$ & 2.2 & 0.7141 & 3.1 & $\begin{array}{r}0.089 \\
0\end{array}$ & $\begin{array}{r}2 . \\
2\end{array}$ & $\begin{array}{r}0.7 \\
0\end{array}$ & 549.7 & 11.4 & 547.2 & 13.1 & 536.6 & 48.4 & 549.7 & 11.4 & $\begin{array}{r}102 . \\
4\end{array}$ \\
\hline $\begin{array}{l}\text { BU0872- } \\
19\end{array}$ & 501 & 21856 & 1.4 & $\begin{array}{r}15.24 \\
48\end{array}$ & 2.6 & 1.1475 & 3.1 & $\begin{array}{r}0.126 \\
9\end{array}$ & $\begin{array}{l}1 . \\
8\end{array}$ & $\begin{array}{r}0.5 \\
7\end{array}$ & 770.0 & 12.8 & 776.1 & 16.8 & 793.5 & 53.5 & 770.0 & 12.8 & 97.0 \\
\hline $\begin{array}{l}\text { BU0872- } \\
22\end{array}$ & 282 & 18836 & 0.9 & $\begin{array}{r}15.14 \\
93\end{array}$ & 1.3 & 1.2118 & 1.6 & $\begin{array}{r}0.133 \\
1\end{array}$ & $\begin{array}{l}0 . \\
8\end{array}$ & $\begin{array}{r}0.5 \\
4\end{array}$ & 805.8 & 6.4 & 806.0 & 8.7 & 806.7 & 27.7 & 805.8 & 6.4 & 99.9 \\
\hline $\begin{array}{l}\text { BU0872- } \\
17\end{array}$ & 345 & 16778 & 2.2 & $\begin{array}{r}14.78 \\
01\end{array}$ & 2.8 & 1.2442 & 5.8 & $\begin{array}{r}0.133 \\
4\end{array}$ & $\begin{array}{r}5 . \\
1\end{array}$ & $\begin{array}{r}0.8 \\
8\end{array}$ & 807.1 & 38.9 & 820.8 & 32.8 & 858.1 & 57.6 & 807.1 & 38.9 & 94.1 \\
\hline $\begin{array}{l}\text { BU0872- } \\
14\end{array}$ & $\begin{array}{r}208 \\
5\end{array}$ & 78854 & 5.4 & $\begin{array}{r}14.66 \\
61\end{array}$ & 3.6 & 1.2989 & 4.3 & $\begin{array}{r}0.138 \\
2\end{array}$ & 2. & $\begin{array}{r}0.5 \\
3 \\
\end{array}$ & 834.3 & 17.8 & 845.2 & 24.6 & 874.2 & 75.4 & 834.3 & 17.8 & 95.4 \\
\hline BU0872-8 & 320 & 15316 & 3.3 & $\begin{array}{r}14.40 \\
44\end{array}$ & 3.6 & 1.3410 & 4.0 & $\begin{array}{r}0.140 \\
1\end{array}$ & $\begin{array}{l}1 . \\
7\end{array}$ & $\begin{array}{r}0.4 \\
3\end{array}$ & 845.2 & 13.7 & 863.7 & 23.3 & 911.3 & 74.4 & 845.2 & 13.7 & 92.7 \\
\hline BU0872-1 & $\begin{array}{r}200 \\
9\end{array}$ & 98618 & 3.0 & $\begin{array}{r}12.66 \\
81\end{array}$ & 1.7 & 2.0095 & 2.5 & $\begin{array}{r}0.184 \\
6\end{array}$ & $\begin{array}{r}1 . \\
9\end{array}$ & $\begin{array}{r}0.7 \\
5\end{array}$ & $\begin{array}{r}1092 . \\
2\end{array}$ & 19.1 & 1118.7 & 17.3 & $\begin{array}{r}1170 . \\
6\end{array}$ & 33.6 & 1170.6 & 33.6 & 93.3 \\
\hline BU0872-3 & 374 & 34454 & 2.3 & $\begin{array}{r}12.57 \\
65\end{array}$ & 2.3 & 2.1698 & 3.5 & $\begin{array}{r}0.197 \\
9\end{array}$ & 2. & $\begin{array}{r}0.7 \\
7\end{array}$ & $\begin{array}{r}1164 . \\
1\end{array}$ & 29.1 & 1171.4 & 24.6 & $\begin{array}{r}1185 . \\
0\end{array}$ & 44.5 & 1185.0 & 44.5 & 98.2 \\
\hline $\begin{array}{l}\text { BU0872- } \\
20\end{array}$ & 673 & 43852 & 1.2 & $\begin{array}{r}12.49 \\
45\end{array}$ & 1.8 & 2.0219 & 2.6 & $\begin{array}{r}0.183 \\
2\end{array}$ & $\begin{array}{r}1 . \\
9\end{array}$ & $\begin{array}{r}0.7 \\
3\end{array}$ & $\begin{array}{r}1084 . \\
6\end{array}$ & 19.3 & 1122.9 & 18.0 & $\begin{array}{r}1197 . \\
9\end{array}$ & 35.7 & 1197.9 & 35.7 & 90.5 \\
\hline $\begin{array}{l}\text { BU0872- } \\
11\end{array}$ & 310 & 20638 & 4.8 & $\begin{array}{r}9.054 \\
4\end{array}$ & 2.0 & 4.6401 & 2.4 & $\begin{array}{r}0.304 \\
7\end{array}$ & $\begin{array}{l}1 . \\
4\end{array}$ & $\begin{array}{r}0.5 \\
9\end{array}$ & $\begin{array}{r}1714 . \\
6\end{array}$ & 21.5 & 1756.5 & 20.2 & $\begin{array}{r}1806 . \\
7\end{array}$ & 35.5 & 1806.7 & 35.5 & 94.9 \\
\hline BU0872-6 & 717 & 86814 & 0.6 & $\begin{array}{r}6.038 \\
5 \\
\end{array}$ & 2.5 & $\begin{array}{r}10.203 \\
9 \\
\end{array}$ & 2.5 & $\begin{array}{r}0.446 \\
9 \\
\end{array}$ & $\begin{array}{r}0 . \\
5\end{array}$ & $\begin{array}{r}0.2 \\
0 \\
\end{array}$ & $\begin{array}{r}2381 . \\
4 \\
\end{array}$ & 10.0 & 2453.4 & 23.2 & $\begin{array}{r}2513 . \\
7 \\
\end{array}$ & 41.4 & 2513.7 & 41.4 & 94.7 \\
\hline $\begin{array}{l}\text { BU08135- } \\
101\end{array}$ & $\begin{array}{r}702 \\
2\end{array}$ & 86340 & 22.9 & $\begin{array}{r}17.05 \\
13\end{array}$ & 2.4 & 0.5170 & 3.4 & $\begin{array}{r}0.063 \\
9\end{array}$ & $\begin{array}{c}2 . \\
4\end{array}$ & $\begin{array}{r}0.7 \\
0\end{array}$ & 399.5 & 9.2 & 423.1 & 11.8 & 554.0 & 53.2 & 399.5 & 9.2 & 72.1 \\
\hline $\begin{array}{l}\text { BU08135- } \\
66\end{array}$ & $\begin{array}{r}533 \\
7\end{array}$ & $\begin{array}{r}14157 \\
0\end{array}$ & 11.0 & $\begin{array}{r}17.90 \\
41\end{array}$ & 1.6 & 0.5209 & 2.0 & $\begin{array}{r}0.067 \\
6\end{array}$ & $\begin{array}{l}1 . \\
3\end{array}$ & $\begin{array}{r}0.6 \\
4\end{array}$ & 421.9 & 5.3 & 425.8 & 7.0 & 446.5 & 34.5 & 421.9 & 5.3 & 94.5 \\
\hline $\begin{array}{l}\text { BU08135- } \\
62\end{array}$ & $\begin{array}{r}128 \\
9 \\
\end{array}$ & 11558 & 1.8 & $\begin{array}{r}16.59 \\
81\end{array}$ & 2.9 & 0.5979 & 3.1 & $\begin{array}{r}0.072 \\
0\end{array}$ & $\begin{array}{r}1 . \\
3\end{array}$ & $\begin{array}{r}0.4 \\
0\end{array}$ & 448.0 & 5.4 & 475.9 & 11.9 & 612.5 & 62.1 & 448.0 & 5.4 & 73.2 \\
\hline $\begin{array}{l}\text { BU08135- } \\
50\end{array}$ & 558 & 13792 & 1.2 & $\begin{array}{r}16.87 \\
62\end{array}$ & 3.0 & 0.6019 & 5.0 & $\begin{array}{r}0.073 \\
7\end{array}$ & $\begin{array}{r}4 . \\
0\end{array}$ & $\begin{array}{r}0.8 \\
0\end{array}$ & 458.2 & 17.8 & 478.4 & 19.1 & 576.5 & 64.6 & 458.2 & 17.8 & 79.5 \\
\hline $\begin{array}{l}\text { BU08135- } \\
70\end{array}$ & 243 & 8724 & 2.4 & $\begin{array}{r}17.42 \\
02\end{array}$ & 2.5 & 0.6031 & 2.9 & $\begin{array}{r}0.076 \\
2\end{array}$ & $\begin{array}{l}1 . \\
5\end{array}$ & $\begin{array}{r}0.5 \\
0\end{array}$ & 473.4 & 6.7 & 479.2 & 11.2 & 507.1 & 55.6 & 473.4 & 6.7 & 93.4 \\
\hline
\end{tabular}




\begin{tabular}{|c|c|c|c|c|c|c|c|c|c|c|c|c|c|c|c|c|c|c|c|}
\hline $\begin{array}{l}\text { BU08135- } \\
13\end{array}$ & 273 & 9350 & 8.6 & $\begin{array}{r}17.84 \\
20 \\
\end{array}$ & 1.8 & 0.5896 & 1.9 & $\begin{array}{r}0.076 \\
3 \\
\end{array}$ & $\begin{array}{r}0 . \\
6 \\
\end{array}$ & $\begin{array}{r}0.3 \\
2 \\
\end{array}$ & 474.0 & 2.8 & 470.6 & 7.1 & 454.3 & 39.7 & 474.0 & 2.8 & $\begin{array}{r}104 . \\
3 \\
\end{array}$ \\
\hline $\begin{array}{l}\text { BU08135- } \\
92\end{array}$ & $\begin{array}{r}105 \\
2\end{array}$ & 30604 & 2.3 & $\begin{array}{r}17.33 \\
26\end{array}$ & 0.9 & 0.6270 & 1.0 & $\begin{array}{r}0.078 \\
8\end{array}$ & $\begin{array}{r}0 . \\
5\end{array}$ & $\begin{array}{r}0.4 \\
9\end{array}$ & 489.1 & 2.4 & 494.2 & 4.0 & 518.2 & 19.8 & 489.1 & 2.4 & 94.4 \\
\hline $\begin{array}{l}\text { BU08135- } \\
25\end{array}$ & $\begin{array}{r}115 \\
9\end{array}$ & 16528 & 2.3 & $\begin{array}{r}16.99 \\
98\end{array}$ & 1.6 & 0.6461 & 2.5 & $\begin{array}{r}0.079 \\
7\end{array}$ & $\begin{array}{r}2 . \\
0\end{array}$ & $\begin{array}{r}0.7 \\
8\end{array}$ & 494.1 & 9.3 & 506.0 & 10.0 & 560.6 & 34.1 & 494.1 & 9.3 & 88.1 \\
\hline $\begin{array}{l}\text { BU08135- } \\
95\end{array}$ & 308 & 9642 & 1.2 & $\begin{array}{r}17.56 \\
85 \\
\end{array}$ & 2.7 & 0.6372 & 3.4 & $\begin{array}{r}0.081 \\
2 \\
\end{array}$ & $\begin{array}{r}2 . \\
0\end{array}$ & $\begin{array}{r}0.6 \\
0 \\
\end{array}$ & 503.2 & 9.8 & 500.6 & 13.4 & 488.4 & 59.8 & 503.2 & 9.8 & $\begin{array}{r}103 . \\
0\end{array}$ \\
\hline $\begin{array}{l}\text { BU08135- } \\
36\end{array}$ & 605 & 16404 & 1.9 & $\begin{array}{r}17.38 \\
45 \\
\end{array}$ & 1.1 & 0.6465 & 1.2 & $\begin{array}{r}0.081 \\
5 \\
\end{array}$ & $\begin{array}{r}0 . \\
6 \\
\end{array}$ & $\begin{array}{r}0.4 \\
7 \\
\end{array}$ & 505.1 & 2.8 & 506.3 & 4.9 & 511.6 & 24.1 & 505.1 & 2.8 & 98.7 \\
\hline $\begin{array}{l}\text { BU08135- } \\
1\end{array}$ & 658 & 27212 & 4.0 & $\begin{array}{r}17.38 \\
91 \\
\end{array}$ & 1.4 & 0.6678 & 1.7 & $\begin{array}{r}0.084 \\
2 \\
\end{array}$ & $\begin{array}{r}1 . \\
0\end{array}$ & $\begin{array}{r}0.6 \\
1 \\
\end{array}$ & 521.3 & 5.2 & 519.4 & 7.0 & 511.0 & 29.9 & 521.3 & 5.2 & $\begin{array}{r}102 . \\
0 \\
\end{array}$ \\
\hline $\begin{array}{l}\text { BU08135- } \\
37\end{array}$ & 776 & 21404 & 2.1 & $\begin{array}{r}17.49 \\
52\end{array}$ & 2.1 & 0.6776 & 2.1 & $\begin{array}{r}0.086 \\
0\end{array}$ & $\begin{array}{r}0 . \\
5\end{array}$ & $\begin{array}{r}0.2 \\
4\end{array}$ & 531.7 & 2.6 & 525.3 & 8.7 & 497.6 & 45.2 & 531.7 & 2.6 & $\begin{array}{r}106 . \\
8\end{array}$ \\
\hline $\begin{array}{l}\text { BU08135- } \\
16\end{array}$ & 336 & 9764 & 3.1 & $\begin{array}{r}16.39 \\
38 \\
\end{array}$ & 1.0 & 0.7402 & 1.9 & $\begin{array}{r}0.088 \\
0 \\
\end{array}$ & $\begin{array}{l}1 . \\
7 \\
\end{array}$ & $\begin{array}{r}0.8 \\
6 \\
\end{array}$ & 543.7 & 8.7 & 562.5 & 8.4 & 639.2 & 21.5 & 543.7 & 8.7 & 85.1 \\
\hline $\begin{array}{l}\text { BU08135- } \\
72\end{array}$ & $\begin{array}{r}133 \\
4 \\
\end{array}$ & 38774 & 10.8 & $\begin{array}{r}16.49 \\
91 \\
\end{array}$ & 1.0 & 0.8100 & 1.3 & $\begin{array}{r}0.096 \\
9 \\
\end{array}$ & $\begin{array}{r}0 . \\
9\end{array}$ & $\begin{array}{r}0.6 \\
4\end{array}$ & 596.4 & 4.8 & 602.5 & 6.0 & 625.4 & 21.8 & 596.4 & 4.8 & 95.4 \\
\hline $\begin{array}{l}\text { BU08135- } \\
82\end{array}$ & $\begin{array}{r}103 \\
6 \\
\end{array}$ & 34818 & 13.6 & $\begin{array}{r}16.23 \\
81 \\
\end{array}$ & 1.0 & 0.8477 & 1.6 & $\begin{array}{r}0.099 \\
8 \\
\end{array}$ & $\begin{array}{r}1 . \\
2\end{array}$ & $\begin{array}{r}0.7 \\
9 \\
\end{array}$ & 613.4 & 7.3 & 623.4 & 7.3 & 659.7 & 20.4 & 613.4 & 7.3 & 93.0 \\
\hline $\begin{array}{l}\text { BU08135- } \\
96\end{array}$ & $\begin{array}{r}479 \\
3\end{array}$ & $\begin{array}{r}11712 \\
0\end{array}$ & 10.1 & $\begin{array}{r}15.50 \\
57\end{array}$ & 1.3 & 0.9519 & 2.0 & $\begin{array}{r}0.107 \\
0\end{array}$ & $\begin{array}{r}1 . \\
5\end{array}$ & $\begin{array}{r}0.7 \\
6\end{array}$ & 655.6 & 9.4 & 679.1 & 9.8 & 757.8 & 27.0 & 655.6 & 9.4 & 86.5 \\
\hline $\begin{array}{l}\text { BU08135- } \\
17\end{array}$ & 278 & 6392 & 1.4 & $\begin{array}{r}15.47 \\
81\end{array}$ & 1.3 & 0.9635 & 2.7 & $\begin{array}{r}0.108 \\
2\end{array}$ & $\begin{array}{r}2 . \\
4\end{array}$ & $\begin{array}{r}0.8 \\
8\end{array}$ & 662.1 & 15.0 & 685.1 & 13.5 & 761.6 & 27.4 & 662.1 & 15.0 & 86.9 \\
\hline $\begin{array}{l}\text { BU08135- } \\
69\end{array}$ & 110 & 6366 & 2.6 & $\begin{array}{r}15.90 \\
87 \\
\end{array}$ & 2.0 & 0.9835 & 2.3 & $\begin{array}{r}0.113 \\
5 \\
\end{array}$ & $\begin{array}{r}1 . \\
2\end{array}$ & $\begin{array}{r}0.5 \\
1 \\
\end{array}$ & 692.9 & 7.8 & 695.4 & 11.8 & 703.4 & 43.0 & 692.9 & 7.8 & 98.5 \\
\hline $\begin{array}{l}\text { BU08135- } \\
77\end{array}$ & 304 & 13604 & 1.4 & $\begin{array}{r}15.56 \\
77 \\
\end{array}$ & 0.8 & 1.0060 & 1.5 & $\begin{array}{r}0.113 \\
6 \\
\end{array}$ & $\begin{array}{r}1 . \\
3 \\
\end{array}$ & $\begin{array}{r}0.8 \\
3\end{array}$ & 693.5 & 8.4 & 706.8 & 7.7 & 749.4 & 17.7 & 693.5 & 8.4 & 92.5 \\
\hline $\begin{array}{l}\text { BU08135- } \\
2\end{array}$ & $\begin{array}{r}109 \\
4 \\
\end{array}$ & 39590 & 12.8 & $\begin{array}{r}14.86 \\
08 \\
\end{array}$ & 1.3 & 1.0696 & 1.5 & $\begin{array}{r}0.115 \\
3 \\
\end{array}$ & $\begin{array}{r}0 . \\
8\end{array}$ & $\begin{array}{r}0.5 \\
5 \\
\end{array}$ & 703.3 & 5.5 & 738.5 & 7.9 & 846.8 & 26.0 & 703.3 & 5.5 & 83.1 \\
\hline $\begin{array}{l}\text { BU08135- } \\
23\end{array}$ & 300 & 14964 & 2.3 & $\begin{array}{r}15.88 \\
34\end{array}$ & 2.3 & 1.0512 & 2.3 & $\begin{array}{r}0.121 \\
1\end{array}$ & $\begin{array}{r}0 . \\
5\end{array}$ & $\begin{array}{r}0.2 \\
2\end{array}$ & 736.9 & 3.5 & 729.5 & 12.0 & 706.8 & 47.9 & 736.9 & 3.5 & $\begin{array}{r}104 . \\
2\end{array}$ \\
\hline $\begin{array}{l}\text { BU08135- } \\
40\end{array}$ & 134 & 5956 & 1.7 & $\begin{array}{r}15.63 \\
19 \\
\end{array}$ & 1.4 & 1.0918 & 1.9 & $\begin{array}{r}0.123 \\
8 \\
\end{array}$ & $\begin{array}{r}1 . \\
3\end{array}$ & $\begin{array}{r}0.7 \\
0 \\
\end{array}$ & 752.3 & 9.4 & 749.4 & 10.0 & 740.7 & 28.6 & 752.3 & 9.4 & $\begin{array}{r}101 . \\
6 \\
\end{array}$ \\
\hline $\begin{array}{l}\text { BU08135- } \\
21\end{array}$ & 276 & 9210 & 1.7 & $\begin{array}{r}15.37 \\
70 \\
\end{array}$ & 2.0 & 1.1227 & 2.2 & $\begin{array}{r}0.125 \\
2 \\
\end{array}$ & $\begin{array}{r}0 . \\
9 \\
\end{array}$ & $\begin{array}{r}0.4 \\
1 \\
\end{array}$ & 760.5 & 6.5 & 764.3 & 11.7 & 775.4 & 41.9 & 760.5 & 6.5 & 98.1 \\
\hline $\begin{array}{l}\text { BU08135- } \\
9\end{array}$ & 107 & 5354 & 0.7 & $\begin{array}{r}15.56 \\
13 \\
\end{array}$ & 1.6 & 1.1341 & 2.1 & $\begin{array}{r}0.128 \\
0 \\
\end{array}$ & $\begin{array}{r}1 . \\
4 \\
\end{array}$ & $\begin{array}{r}0.6 \\
6 \\
\end{array}$ & 776.4 & 10.1 & 769.7 & 11.3 & 750.2 & 33.2 & 776.4 & 10.1 & $\begin{array}{r}103 . \\
5 \\
\end{array}$ \\
\hline $\begin{array}{l}\text { BU08135- } \\
28\end{array}$ & 291 & 18624 & 1.6 & $\begin{array}{r}15.26 \\
72\end{array}$ & 1.1 & 1.1606 & 1.6 & $\begin{array}{r}0.128 \\
5\end{array}$ & $\begin{array}{r}1 . \\
2\end{array}$ & $\begin{array}{r}0.7 \\
4\end{array}$ & 779.3 & 8.6 & 782.2 & 8.7 & 790.4 & 22.5 & 779.3 & 8.6 & 98.6 \\
\hline $\begin{array}{l}\text { BU08135- } \\
71\end{array}$ & 260 & 13158 & 2.5 & $\begin{array}{r}13.67 \\
38\end{array}$ & 2.1 & 1.3606 & 3.0 & $\begin{array}{r}0.134 \\
9\end{array}$ & $\begin{array}{r}2 . \\
1\end{array}$ & $\begin{array}{r}0.7 \\
1\end{array}$ & 816.0 & 16.3 & 872.1 & 17.6 & $\begin{array}{r}1017 . \\
6\end{array}$ & 42.9 & 816.0 & 16.3 & 80.2 \\
\hline $\begin{array}{l}\text { BU08135- } \\
39\end{array}$ & 363 & 18360 & 5.1 & $\begin{array}{r}14.95 \\
90\end{array}$ & 0.8 & 1.2954 & 0.9 & $\begin{array}{r}0.140 \\
5\end{array}$ & $\begin{array}{r}0 . \\
5\end{array}$ & $\begin{array}{r}0.5 \\
3\end{array}$ & 847.7 & 4.0 & 843.7 & 5.4 & 833.1 & 16.6 & 847.7 & 4.0 & $\begin{array}{r}101 . \\
8\end{array}$ \\
\hline $\begin{array}{l}\text { BU08135- } \\
8\end{array}$ & $\begin{array}{r}108 \\
9 \\
\end{array}$ & 49060 & 3.2 & $\begin{array}{r}13.64 \\
95 \\
\end{array}$ & 1.4 & 1.4447 & 1.7 & $\begin{array}{r}0.143 \\
0 \\
\end{array}$ & $\begin{array}{r}1 . \\
1\end{array}$ & $\begin{array}{r}0.6 \\
2 \\
\end{array}$ & 861.7 & 8.7 & 907.7 & 10.4 & $\begin{array}{r}1021 . \\
2 \\
\end{array}$ & 27.6 & 861.7 & 8.7 & 84.4 \\
\hline $\begin{array}{l}\text { BU08135- } \\
67\end{array}$ & 585 & 42350 & 7.6 & $\begin{array}{r}13.49 \\
57 \\
\end{array}$ & 2.3 & 1.5017 & 5.2 & $\begin{array}{r}0.147 \\
0 \\
\end{array}$ & $\begin{array}{r}4 . \\
7 \\
\end{array}$ & $\begin{array}{r}0.9 \\
0 \\
\end{array}$ & 884.0 & 38.7 & 931.1 & 31.8 & $\begin{array}{r}1044 . \\
1 \\
\end{array}$ & 45.8 & 884.0 & 38.7 & 84.7 \\
\hline BU08135- & 504 & 30914 & 10.0 & 13.80 & 1.0 & 1.5975 & 1.5 & 0.159 & 1. & 0.7 & 956.2 & 9.6 & 969.2 & 9.1 & 998.8 & 19.7 & 998.8 & 19.7 & 95.7 \\
\hline
\end{tabular}




\begin{tabular}{|c|c|c|c|c|c|c|c|c|c|c|c|c|c|c|c|c|c|c|c|}
\hline 75 & & & & 15 & & & & 9 & 1 & 4 & & & & & & & & & \\
\hline $\begin{array}{l}\text { BU08135- } \\
73\end{array}$ & 342 & 21936 & 2.8 & $\begin{array}{r}13.79 \\
18 \\
\end{array}$ & 1.4 & 1.6028 & 1.5 & $\begin{array}{r}0.160 \\
3 \\
\end{array}$ & $\begin{array}{l}0 . \\
7\end{array}$ & $\begin{array}{r}0.4 \\
5 \\
\end{array}$ & 958.5 & 6.2 & 971.3 & 9.6 & $\begin{array}{r}1000 . \\
2 \\
\end{array}$ & 27.9 & 1000.2 & 27.9 & 95.8 \\
\hline $\begin{array}{l}\text { BU08135- } \\
99\end{array}$ & 167 & 10246 & 2.0 & $\begin{array}{r}13.75 \\
86 \\
\end{array}$ & 1.7 & 1.7318 & 1.8 & $\begin{array}{r}0.172 \\
8 \\
\end{array}$ & $\begin{array}{l}0 . \\
6 \\
\end{array}$ & $\begin{array}{r}0.3 \\
2 \\
\end{array}$ & $\begin{array}{r}1027 . \\
6 \\
\end{array}$ & 5.4 & 1020.4 & 11.6 & $\begin{array}{r}1005 . \\
1 \\
\end{array}$ & 34.7 & 1005.1 & 34.7 & $\begin{array}{r}102 . \\
2 \\
\end{array}$ \\
\hline $\begin{array}{l}\text { BU08135- } \\
5\end{array}$ & 403 & 31936 & 1.0 & $\begin{array}{r}13.53 \\
74 \\
\end{array}$ & 2.9 & 1.8496 & 3.4 & $\begin{array}{r}0.181 \\
6 \\
\end{array}$ & $\begin{array}{l}1 . \\
7\end{array}$ & $\begin{array}{r}0.5 \\
1 \\
\end{array}$ & $\begin{array}{r}1075 . \\
7 \\
\end{array}$ & 17.1 & 1063.3 & 22.2 & $\begin{array}{r}1037 . \\
9 \\
\end{array}$ & 58.4 & 1037.9 & 58.4 & $\begin{array}{r}103 . \\
6 \\
\end{array}$ \\
\hline $\begin{array}{l}\text { BU08135- } \\
84\end{array}$ & 600 & 33888 & 3.0 & $\begin{array}{r}13.52 \\
28 \\
\end{array}$ & 0.8 & 1.6382 & 1.0 & $\begin{array}{r}0.160 \\
7 \\
\end{array}$ & $\begin{array}{r}0 . \\
5\end{array}$ & $\begin{array}{r}0.5 \\
3 \\
\end{array}$ & 960.5 & 4.6 & 985.0 & 6.2 & $\begin{array}{r}1040 . \\
1 \\
\end{array}$ & 16.8 & 1040.1 & 16.8 & 92.4 \\
\hline $\begin{array}{l}\text { BU08135- } \\
12\end{array}$ & 50 & 3472 & 0.5 & $\begin{array}{r}13.47 \\
79 \\
\end{array}$ & 2.6 & 1.8796 & 4.7 & $\begin{array}{r}0.183 \\
7 \\
\end{array}$ & $\begin{array}{r}3 . \\
8 \\
\end{array}$ & $\begin{array}{r}0.8 \\
2 \\
\end{array}$ & $\begin{array}{r}1087 . \\
3 \\
\end{array}$ & 38.3 & 1073.9 & 30.8 & $\begin{array}{r}1046 . \\
8 \\
\end{array}$ & 53.2 & 1046.8 & 53.2 & $\begin{array}{r}103 . \\
9 \\
\end{array}$ \\
\hline $\begin{array}{l}\text { BU08135- } \\
55\end{array}$ & 372 & 23588 & 7.3 & $\begin{array}{r}13.27 \\
08 \\
\end{array}$ & 1.1 & 1.8486 & 1.5 & $\begin{array}{r}0.177 \\
9 \\
\end{array}$ & $\begin{array}{r}1 . \\
0\end{array}$ & $\begin{array}{r}0.6 \\
5 \\
\end{array}$ & $\begin{array}{r}1055 . \\
7 \\
\end{array}$ & 9.3 & 1062.9 & 9.6 & $\begin{array}{r}1077 . \\
9 \\
\end{array}$ & 22.3 & 1077.9 & 22.3 & 97.9 \\
\hline $\begin{array}{l}\text { BU08135- } \\
35\end{array}$ & 114 & 8220 & 1.1 & $\begin{array}{r}13.20 \\
91 \\
\end{array}$ & 1.0 & 1.9427 & 1.2 & $\begin{array}{r}0.186 \\
1 \\
\end{array}$ & $\begin{array}{c}0 . \\
6 \\
\end{array}$ & $\begin{array}{r}0.4 \\
7 \\
\end{array}$ & $\begin{array}{r}1100 . \\
3\end{array}$ & 5.7 & 1095.9 & 8.0 & $\begin{array}{r}1087 . \\
3 \\
\end{array}$ & 21.0 & 1087.3 & 21.0 & $\begin{array}{r}101 . \\
2 \\
\end{array}$ \\
\hline $\begin{array}{l}\text { BU08135- } \\
79\end{array}$ & 54 & 3810 & 1.0 & $\begin{array}{r}13.12 \\
01 \\
\end{array}$ & 3.6 & 1.9651 & 3.8 & $\begin{array}{r}0.187 \\
0 \\
\end{array}$ & $\begin{array}{l}1 . \\
4 \\
\end{array}$ & $\begin{array}{r}0.3 \\
6 \\
\end{array}$ & $\begin{array}{r}1105 . \\
0 \\
\end{array}$ & 14.2 & 1103.6 & 25.8 & $\begin{array}{r}1100 . \\
8 \\
\end{array}$ & 71.6 & 1100.8 & 71.6 & $\begin{array}{r}100 . \\
4 \\
\end{array}$ \\
\hline $\begin{array}{l}\text { BU08135- } \\
63\end{array}$ & 192 & 16082 & 0.8 & $\begin{array}{r}13.10 \\
23 \\
\end{array}$ & 1.2 & 1.8819 & 1.7 & $\begin{array}{r}0.178 \\
8 \\
\end{array}$ & $\begin{array}{c}1 . \\
1\end{array}$ & $\begin{array}{r}0.6 \\
7 \\
\end{array}$ & $\begin{array}{r}1060 . \\
6 \\
\end{array}$ & 10.9 & 1074.7 & 11.0 & $\begin{array}{r}1103 . \\
6 \\
\end{array}$ & 24.5 & 1103.6 & 24.5 & 96.1 \\
\hline $\begin{array}{l}\text { BU08135- } \\
26\end{array}$ & 305 & 21746 & 1.6 & $\begin{array}{r}13.05 \\
11 \\
\end{array}$ & 1.3 & 1.9287 & 2.2 & $\begin{array}{r}0.182 \\
6 \\
\end{array}$ & $\begin{array}{r}1 . \\
8\end{array}$ & $\begin{array}{r}0.8 \\
1 \\
\end{array}$ & $\begin{array}{r}1081 . \\
0 \\
\end{array}$ & 18.1 & 1091.1 & 15.0 & $\begin{array}{r}1111 . \\
3 \\
\end{array}$ & 26.4 & 1111.3 & 26.4 & 97.3 \\
\hline $\begin{array}{l}\text { BU08135- } \\
31\end{array}$ & 830 & 53678 & 10.2 & $\begin{array}{r}13.04 \\
50 \\
\end{array}$ & 1.2 & 1.9888 & 1.5 & $\begin{array}{r}0.188 \\
2 \\
\end{array}$ & $\begin{array}{r}0 . \\
8 \\
\end{array}$ & $\begin{array}{r}0.5 \\
5\end{array}$ & $\begin{array}{r}1111 . \\
4\end{array}$ & 8.2 & 1111.7 & 9.8 & $\begin{array}{r}1112 . \\
3\end{array}$ & 24.2 & 1112.3 & 24.2 & 99.9 \\
\hline $\begin{array}{l}\text { BU08135- } \\
11\end{array}$ & $\begin{array}{r}205 \\
8 \\
\end{array}$ & $\begin{array}{r}10532 \\
6 \\
\end{array}$ & 4.5 & $\begin{array}{r}13.04 \\
29 \\
\end{array}$ & 1.7 & 1.6694 & 3.5 & $\begin{array}{r}0.157 \\
9 \\
\end{array}$ & $\begin{array}{r}3 . \\
1\end{array}$ & $\begin{array}{r}0.8 \\
8 \\
\end{array}$ & 945.2 & 27.3 & 997.0 & 22.4 & $\begin{array}{r}1112 . \\
6 \\
\end{array}$ & 33.4 & 1112.6 & 33.4 & 85.0 \\
\hline $\begin{array}{l}\text { BU08135- } \\
88\end{array}$ & 335 & 23376 & 9.6 & $\begin{array}{r}13.01 \\
29 \\
\end{array}$ & 1.3 & 1.9704 & 1.4 & $\begin{array}{r}0.186 \\
0 \\
\end{array}$ & $\begin{array}{l}0 . \\
6 \\
\end{array}$ & $\begin{array}{r}0.4 \\
2 \\
\end{array}$ & $\begin{array}{r}1099 . \\
5 \\
\end{array}$ & 6.1 & 1105.4 & 9.6 & $\begin{array}{r}1117 . \\
2 \\
\end{array}$ & 26.0 & 1117.2 & 26.0 & 98.4 \\
\hline $\begin{array}{l}\text { BU08135- } \\
93\end{array}$ & 798 & 19968 & 2.5 & $\begin{array}{r}13.01 \\
24 \\
\end{array}$ & 1.4 & 1.9155 & 2.1 & $\begin{array}{r}0.180 \\
8 \\
\end{array}$ & $\begin{array}{c}1 . \\
5 \\
\end{array}$ & $\begin{array}{r}0.7 \\
2 \\
\end{array}$ & $\begin{array}{r}1071 . \\
2 \\
\end{array}$ & 14.6 & 1086.5 & 13.8 & $\begin{array}{r}1117 . \\
3 \\
\end{array}$ & 28.8 & 1117.3 & 28.8 & 95.9 \\
\hline $\begin{array}{l}\text { BU08135- } \\
68\end{array}$ & 414 & 34372 & 4.0 & $\begin{array}{r}13.00 \\
42 \\
\end{array}$ & 1.7 & 1.7408 & 2.2 & $\begin{array}{r}0.164 \\
2 \\
\end{array}$ & $\begin{array}{r}1 . \\
4\end{array}$ & $\begin{array}{r}0.6 \\
2 \\
\end{array}$ & 980.0 & 12.4 & 1023.8 & 14.2 & $\begin{array}{r}1118 . \\
6 \\
\end{array}$ & 34.3 & 1118.6 & 34.3 & 87.6 \\
\hline $\begin{array}{l}\text { BU08135- } \\
51\end{array}$ & 338 & 21776 & 5.9 & $\begin{array}{r}12.95 \\
16 \\
\end{array}$ & 1.3 & 1.7909 & 1.9 & $\begin{array}{r}0.168 \\
2 \\
\end{array}$ & $\begin{array}{l}1 . \\
3\end{array}$ & $\begin{array}{r}0.7 \\
0 \\
\end{array}$ & $\begin{array}{r}1002 . \\
3 \\
\end{array}$ & 12.2 & 1042.2 & 12.2 & $\begin{array}{r}1126 . \\
6 \\
\end{array}$ & 26.5 & 1126.6 & 26.5 & 89.0 \\
\hline $\begin{array}{l}\text { BU08135- } \\
34\end{array}$ & 447 & 44094 & 4.1 & $\begin{array}{r}12.92 \\
98 \\
\end{array}$ & 1.1 & 2.0371 & 2.4 & $\begin{array}{r}0.191 \\
0 \\
\end{array}$ & $\begin{array}{r}2 . \\
2 \\
\end{array}$ & $\begin{array}{r}0.9 \\
0 \\
\end{array}$ & $\begin{array}{r}1126 . \\
9 \\
\end{array}$ & 22.2 & 1128.0 & 16.4 & $\begin{array}{r}1130 . \\
0 \\
\end{array}$ & 21.4 & 1130.0 & 21.4 & 99.7 \\
\hline $\begin{array}{l}\text { BU08135- } \\
97\end{array}$ & $\begin{array}{r}112 \\
3 \\
\end{array}$ & 16826 & 34.6 & $\begin{array}{r}12.92 \\
94 \\
\end{array}$ & 2.1 & 1.4452 & 2.2 & $\begin{array}{r}0.135 \\
5 \\
\end{array}$ & $\begin{array}{r}0 . \\
9 \\
\end{array}$ & $\begin{array}{r}0.4 \\
0 \\
\end{array}$ & 819.3 & 6.8 & 907.9 & 13.4 & $\begin{array}{r}1130 . \\
1 \\
\end{array}$ & 40.9 & 1130.1 & 40.9 & 72.5 \\
\hline $\begin{array}{l}\text { BU08135- } \\
15\end{array}$ & 397 & 30058 & 2.8 & $\begin{array}{r}12.92 \\
42 \\
\end{array}$ & 0.9 & 1.9695 & 1.7 & $\begin{array}{r}0.184 \\
6 \\
\end{array}$ & $\begin{array}{r}1 . \\
5 \\
\end{array}$ & $\begin{array}{r}0.8 \\
5 \\
\end{array}$ & $\begin{array}{r}1092 . \\
1 \\
\end{array}$ & 14.7 & 1105.1 & 11.6 & $\begin{array}{r}1130 . \\
8 \\
\end{array}$ & 18.2 & 1130.8 & 18.2 & 96.6 \\
\hline $\begin{array}{l}\text { BU08135- } \\
56\end{array}$ & 490 & 32294 & 6.9 & $\begin{array}{r}12.88 \\
37 \\
\end{array}$ & 1.2 & 1.9674 & 1.4 & $\begin{array}{r}0.183 \\
8 \\
\end{array}$ & $\begin{array}{l}0 . \\
7 \\
\end{array}$ & $\begin{array}{r}0.4 \\
9 \\
\end{array}$ & $\begin{array}{r}1087 . \\
9 \\
\end{array}$ & 6.7 & 1104.4 & 9.1 & $\begin{array}{r}1137 . \\
1 \\
\end{array}$ & 23.5 & 1137.1 & 23.5 & 95.7 \\
\hline $\begin{array}{l}\text { BU08135- } \\
41\end{array}$ & 239 & 16600 & 1.5 & $\begin{array}{r}12.84 \\
76 \\
\end{array}$ & 1.4 & 2.0586 & 1.5 & $\begin{array}{r}0.191 \\
8 \\
\end{array}$ & $\begin{array}{r}0 . \\
5 \\
\end{array}$ & $\begin{array}{r}0.3 \\
3 \\
\end{array}$ & $\begin{array}{r}1131 . \\
2 \\
\end{array}$ & 5.3 & 1135.1 & 10.4 & $\begin{array}{r}1142 . \\
7 \\
\end{array}$ & 28.6 & 1142.7 & 28.6 & 99.0 \\
\hline $\begin{array}{l}\text { BU08135- } \\
32\end{array}$ & 463 & 41744 & 4.4 & $\begin{array}{r}12.84 \\
21 \\
\end{array}$ & 0.8 & 1.7990 & 1.0 & $\begin{array}{r}0.167 \\
6 \\
\end{array}$ & $\begin{array}{c}0 . \\
5 \\
\end{array}$ & $\begin{array}{r}0.5 \\
6 \\
\end{array}$ & 998.6 & 5.0 & 1045.1 & 6.3 & $\begin{array}{r}1143 . \\
5 \\
\end{array}$ & 15.9 & 1143.5 & 15.9 & 87.3 \\
\hline $\begin{array}{l}\text { BU08135- } \\
89\end{array}$ & 442 & 28040 & 4.5 & $\begin{array}{r}12.82 \\
63 \\
\end{array}$ & 2.1 & 1.9226 & 2.7 & $\begin{array}{r}0.178 \\
8 \\
\end{array}$ & $\begin{array}{l}1 . \\
7\end{array}$ & $\begin{array}{r}0.6 \\
2 \\
\end{array}$ & $\begin{array}{r}1060 . \\
7 \\
\end{array}$ & 16.6 & 1089.0 & 18.2 & $\begin{array}{r}1146 . \\
0 \\
\end{array}$ & 42.3 & 1146.0 & 42.3 & 92.6 \\
\hline $\begin{array}{l}\text { BU08135- } \\
100\end{array}$ & 58 & 5298 & 0.9 & $\begin{array}{r}12.82 \\
43 \\
\end{array}$ & 1.8 & 2.1043 & 2.0 & $\begin{array}{r}0.195 \\
7 \\
\end{array}$ & $\begin{array}{r}0 . \\
9\end{array}$ & $\begin{array}{r}0.4 \\
3 \\
\end{array}$ & $\begin{array}{r}1152 . \\
3 \\
\end{array}$ & 9.0 & 1150.2 & 13.7 & $\begin{array}{r}1146 . \\
3 \\
\end{array}$ & 35.7 & 1146.3 & 35.7 & $\begin{array}{r}100 . \\
5 \\
\end{array}$ \\
\hline
\end{tabular}




\begin{tabular}{|c|c|c|c|c|c|c|c|c|c|c|c|c|c|c|c|c|c|c|c|}
\hline $\begin{array}{l}\text { BU08135- } \\
90\end{array}$ & 999 & 59746 & 12.3 & $\begin{array}{r}12.81 \\
88 \\
\end{array}$ & 1.0 & 1.8846 & 1.1 & $\begin{array}{r}0.175 \\
2 \\
\end{array}$ & $\begin{array}{r}0 . \\
5\end{array}$ & $\begin{array}{r}0.4 \\
5 \\
\end{array}$ & $\begin{array}{r}1040 . \\
7 \\
\end{array}$ & 4.8 & 1075.7 & 7.3 & $\begin{array}{r}1147 . \\
1 \\
\end{array}$ & 19.5 & 1147.1 & 19.5 & 90.7 \\
\hline $\begin{array}{l}\text { BU08135- } \\
76\end{array}$ & 108 & 6862 & 1.1 & $\begin{array}{r}12.81 \\
67\end{array}$ & 1.6 & 1.8383 & 1.8 & $\begin{array}{r}0.170 \\
9\end{array}$ & $\begin{array}{r}0 . \\
6\end{array}$ & $\begin{array}{r}0.3 \\
5\end{array}$ & $\begin{array}{r}1017 . \\
0\end{array}$ & 5.8 & 1059.3 & 11.5 & $\begin{array}{r}1147 . \\
5\end{array}$ & 32.6 & 1147.5 & 32.6 & 88.6 \\
\hline $\begin{array}{l}\text { BU08135- } \\
18\end{array}$ & $\begin{array}{r}139 \\
7\end{array}$ & 75262 & 7.8 & $\begin{array}{r}12.70 \\
97\end{array}$ & 1.1 & 1.8833 & 1.5 & $\begin{array}{r}0.173 \\
6\end{array}$ & $\begin{array}{r}1 . \\
0\end{array}$ & $\begin{array}{r}0.6 \\
8\end{array}$ & $\begin{array}{r}1031 . \\
9\end{array}$ & 9.5 & 1075.2 & 9.8 & $\begin{array}{r}1164 . \\
1\end{array}$ & 21.4 & 1164.1 & 21.4 & 88.6 \\
\hline $\begin{array}{l}\text { BU08135- } \\
7\end{array}$ & 430 & 19368 & 3.7 & $\begin{array}{r}12.68 \\
71 \\
\end{array}$ & 2.8 & 1.9610 & 2.9 & $\begin{array}{r}0.180 \\
4 \\
\end{array}$ & $\begin{array}{r}0 . \\
6\end{array}$ & $\begin{array}{r}0.2 \\
1 \\
\end{array}$ & $\begin{array}{r}1069 . \\
4\end{array}$ & 6.1 & 1102.2 & 19.4 & $\begin{array}{r}1167 . \\
6\end{array}$ & 55.8 & 1167.6 & 55.8 & 91.6 \\
\hline $\begin{array}{l}\text { BU08135- } \\
38\end{array}$ & 229 & 16522 & 1.5 & $\begin{array}{r}12.68 \\
64 \\
\end{array}$ & 1.4 & 2.1312 & 1.5 & $\begin{array}{r}0.196 \\
1 \\
\end{array}$ & $\begin{array}{c}0 . \\
5 \\
\end{array}$ & $\begin{array}{r}0.3 \\
4 \\
\end{array}$ & $\begin{array}{r}1154 . \\
3 \\
\end{array}$ & 5.3 & 1159.0 & 10.0 & $\begin{array}{r}1167 . \\
7 \\
\end{array}$ & 27.0 & 1167.7 & 27.0 & 98.9 \\
\hline $\begin{array}{l}\text { BU08135- } \\
46\end{array}$ & 302 & 20710 & 1.5 & $\begin{array}{r}12.68 \\
37 \\
\end{array}$ & 1.0 & 1.9961 & 3.3 & $\begin{array}{r}0.183 \\
6 \\
\end{array}$ & $\begin{array}{r}3 . \\
2\end{array}$ & $\begin{array}{r}0.9 \\
5 \\
\end{array}$ & $\begin{array}{r}1086 . \\
7\end{array}$ & 31.6 & 1114.2 & 22.5 & $\begin{array}{r}1168 . \\
1\end{array}$ & 20.2 & 1168.1 & 20.2 & 93.0 \\
\hline $\begin{array}{l}\text { BU08135- } \\
86\end{array}$ & 867 & 56566 & 1.7 & $\begin{array}{r}12.66 \\
60\end{array}$ & 1.1 & 1.9708 & 1.3 & $\begin{array}{r}0.181 \\
0\end{array}$ & $\begin{array}{r}0 . \\
7\end{array}$ & $\begin{array}{r}0.5 \\
6\end{array}$ & $\begin{array}{r}1072 . \\
7\end{array}$ & 7.2 & 1105.6 & 8.8 & $\begin{array}{r}1170 . \\
9\end{array}$ & 21.6 & 1170.9 & 21.6 & 91.6 \\
\hline $\begin{array}{l}\text { BU08135- } \\
58\end{array}$ & 304 & 18620 & 1.3 & $\begin{array}{r}12.64 \\
77 \\
\end{array}$ & 4.2 & 1.8987 & 4.3 & $\begin{array}{r}0.174 \\
2 \\
\end{array}$ & $\begin{array}{r}1 . \\
2\end{array}$ & $\begin{array}{r}0.2 \\
8 \\
\end{array}$ & $\begin{array}{r}1035 . \\
0 \\
\end{array}$ & 11.5 & 1080.6 & 28.7 & $\begin{array}{r}1173 . \\
8 \\
\end{array}$ & 82.2 & 1173.8 & 82.2 & 88.2 \\
\hline $\begin{array}{l}\text { BU08135- } \\
10\end{array}$ & 145 & 7824 & 1.6 & $\begin{array}{r}12.62 \\
79 \\
\end{array}$ & 5.1 & 1.9085 & 5.2 & $\begin{array}{r}0.174 \\
8 \\
\end{array}$ & $\begin{array}{r}0 . \\
6 \\
\end{array}$ & $\begin{array}{r}0.1 \\
2 \\
\end{array}$ & $\begin{array}{r}1038 . \\
5 \\
\end{array}$ & 6.0 & 1084.1 & 34.5 & $\begin{array}{r}1176 . \\
9 \\
\end{array}$ & $\begin{array}{r}101 . \\
8 \\
\end{array}$ & 1176.9 & $\begin{array}{r}101 . \\
8 \\
\end{array}$ & 88.2 \\
\hline $\begin{array}{l}\text { BU08135- } \\
19\end{array}$ & 137 & 9732 & 0.8 & $\begin{array}{r}12.61 \\
40 \\
\end{array}$ & 2.3 & 2.1312 & 4.1 & $\begin{array}{r}0.195 \\
0 \\
\end{array}$ & $\begin{array}{r}3 . \\
4 \\
\end{array}$ & $\begin{array}{r}0.8 \\
3 \\
\end{array}$ & $\begin{array}{r}1148 . \\
3 \\
\end{array}$ & 36.0 & 1159.0 & 28.5 & $\begin{array}{r}1179 . \\
1 \\
\end{array}$ & 45.5 & 1179.1 & 45.5 & 97.4 \\
\hline $\begin{array}{l}\text { BU08135- } \\
61\end{array}$ & 524 & 40310 & 1.7 & $\begin{array}{r}12.60 \\
38\end{array}$ & 1.1 & 2.1089 & 1.2 & $\begin{array}{r}0.192 \\
8\end{array}$ & $\begin{array}{r}0 . \\
5\end{array}$ & $\begin{array}{r}0.4 \\
3\end{array}$ & $\begin{array}{r}1136 . \\
4\end{array}$ & 5.2 & 1151.7 & 8.0 & $\begin{array}{r}1180 . \\
7\end{array}$ & 20.8 & 1180.7 & 20.8 & 96.2 \\
\hline $\begin{array}{l}\text { BU08135- } \\
81\end{array}$ & 316 & 22600 & 1.7 & $\begin{array}{r}12.58 \\
80\end{array}$ & 1.0 & 1.9724 & 1.2 & $\begin{array}{r}0.180 \\
1\end{array}$ & $\begin{array}{r}0 . \\
8\end{array}$ & $\begin{array}{r}0.6 \\
3\end{array}$ & $\begin{array}{r}1067 . \\
4\end{array}$ & 7.7 & 1106.1 & 8.4 & $\begin{array}{r}1183 . \\
2\end{array}$ & 19.2 & 1183.2 & 19.2 & 90.2 \\
\hline $\begin{array}{l}\text { BU08135- } \\
48\end{array}$ & 436 & 27362 & 1.8 & $\begin{array}{r}12.58 \\
68 \\
\end{array}$ & 1.0 & 2.1312 & 1.7 & $\begin{array}{r}0.194 \\
6 \\
\end{array}$ & $\begin{array}{r}1 . \\
3\end{array}$ & $\begin{array}{r}0.8 \\
1 \\
\end{array}$ & $\begin{array}{r}1146 . \\
0\end{array}$ & 14.1 & 1159.0 & 11.5 & $\begin{array}{r}1183 . \\
3\end{array}$ & 19.4 & 1183.3 & 19.4 & 96.8 \\
\hline $\begin{array}{l}\text { BU08135- } \\
44\end{array}$ & 399 & 27082 & 0.6 & $\begin{array}{r}12.54 \\
06 \\
\end{array}$ & 1.3 & 2.1037 & 1.4 & $\begin{array}{r}0.191 \\
3 \\
\end{array}$ & $\begin{array}{r}0 . \\
6 \\
\end{array}$ & $\begin{array}{r}0.4 \\
3 \\
\end{array}$ & $\begin{array}{r}1128 . \\
6 \\
\end{array}$ & 6.4 & 1150.0 & 10.0 & $\begin{array}{r}1190 . \\
6 \\
\end{array}$ & 25.9 & 1190.6 & 25.9 & 94.8 \\
\hline $\begin{array}{l}\text { BU08135- } \\
53\end{array}$ & 324 & 32720 & 1.1 & $\begin{array}{r}12.50 \\
09 \\
\end{array}$ & 1.5 & 2.1456 & 1.9 & $\begin{array}{r}0.194 \\
5 \\
\end{array}$ & $\begin{array}{r}1 . \\
2\end{array}$ & $\begin{array}{r}0.6 \\
2 \\
\end{array}$ & $\begin{array}{r}1145 . \\
9 \\
\end{array}$ & 12.2 & 1163.6 & 13.0 & $\begin{array}{r}1196 . \\
9 \\
\end{array}$ & 29.2 & 1196.9 & 29.2 & 95.7 \\
\hline $\begin{array}{l}\text { BU08135- } \\
45\end{array}$ & $\begin{array}{r}244 \\
2\end{array}$ & $\begin{array}{r}13953 \\
0\end{array}$ & $\begin{array}{r}156 . \\
2\end{array}$ & $\begin{array}{r}12.46 \\
01\end{array}$ & 1.1 & 2.2567 & 1.2 & $\begin{array}{r}0.203 \\
9\end{array}$ & $\begin{array}{r}0 . \\
5\end{array}$ & $\begin{array}{r}0.4 \\
2\end{array}$ & $\begin{array}{r}1196 . \\
4\end{array}$ & 5.5 & 1198.9 & 8.4 & $\begin{array}{r}1203 . \\
3\end{array}$ & 21.3 & 1203.3 & 21.3 & 99.4 \\
\hline $\begin{array}{l}\text { BU08135- } \\
49\end{array}$ & 442 & 30226 & 1.4 & $\begin{array}{r}12.43 \\
64 \\
\end{array}$ & 0.5 & 2.1632 & 0.7 & $\begin{array}{r}0.195 \\
1 \\
\end{array}$ & $\begin{array}{r}0 . \\
5\end{array}$ & $\begin{array}{r}0.7 \\
0 \\
\end{array}$ & $\begin{array}{r}1149 . \\
0 \\
\end{array}$ & 5.3 & 1169.3 & 4.9 & $\begin{array}{r}1207 . \\
1 \\
\end{array}$ & 10.0 & 1207.1 & 10.0 & 95.2 \\
\hline $\begin{array}{l}\text { BU08135- } \\
74\end{array}$ & 149 & 13094 & 1.2 & $\begin{array}{r}12.28 \\
40 \\
\end{array}$ & 1.3 & 2.3731 & 1.8 & $\begin{array}{r}0.211 \\
4 \\
\end{array}$ & $\begin{array}{r}1 . \\
2 \\
\end{array}$ & $\begin{array}{r}0.6 \\
7 \\
\end{array}$ & $\begin{array}{r}1236 . \\
4 \\
\end{array}$ & 13.4 & 1234.5 & 12.7 & $\begin{array}{r}1231 . \\
3 \\
\end{array}$ & 25.9 & 1231.3 & 25.9 & $\begin{array}{r}100 . \\
4 \\
\end{array}$ \\
\hline $\begin{array}{l}\text { BU08135- } \\
20\end{array}$ & 647 & 48932 & 1.4 & $\begin{array}{r}12.25 \\
93 \\
\end{array}$ & 2.8 & 2.1467 & 2.9 & $\begin{array}{r}0.190 \\
9 \\
\end{array}$ & $\begin{array}{r}0 . \\
9 \\
\end{array}$ & $\begin{array}{r}0.3 \\
1 \\
\end{array}$ & $\begin{array}{r}1126 . \\
1 \\
\end{array}$ & 9.2 & 1164.0 & 20.2 & $\begin{array}{r}1235 . \\
2 \\
\end{array}$ & 54.3 & 1235.2 & 54.3 & 91.2 \\
\hline $\begin{array}{l}\text { BU08135- } \\
52\end{array}$ & 328 & 29804 & 1.1 & $\begin{array}{r}12.09 \\
76\end{array}$ & 3.5 & 2.5093 & 3.7 & $\begin{array}{r}0.220 \\
2\end{array}$ & $\begin{array}{r}1 . \\
3\end{array}$ & $\begin{array}{r}0.3 \\
3\end{array}$ & $\begin{array}{r}1282 . \\
8\end{array}$ & 14.5 & 1274.7 & 27.2 & $\begin{array}{r}1261 . \\
2\end{array}$ & 69.0 & 1261.2 & 69.0 & $\begin{array}{r}101 . \\
7\end{array}$ \\
\hline $\begin{array}{l}\text { BU08135- } \\
104\end{array}$ & 619 & 58086 & 3.8 & $\begin{array}{r}12.08 \\
16\end{array}$ & 1.0 & 2.4317 & 1.1 & $\begin{array}{r}0.213 \\
1\end{array}$ & $\begin{array}{r}0 . \\
5\end{array}$ & $\begin{array}{r}0.4 \\
4\end{array}$ & $\begin{array}{r}1245 . \\
2\end{array}$ & 5.7 & 1252.0 & 8.1 & $\begin{array}{r}1263 . \\
8\end{array}$ & 19.7 & 1263.8 & 19.7 & 98.5 \\
\hline $\begin{array}{l}\text { BU08135- } \\
57\end{array}$ & 198 & 13854 & 2.0 & $\begin{array}{r}12.01 \\
94\end{array}$ & 1.1 & 2.1835 & 1.4 & $\begin{array}{r}0.190 \\
3\end{array}$ & $\begin{array}{r}0 . \\
9\end{array}$ & $\begin{array}{r}0.6 \\
4\end{array}$ & $\begin{array}{r}1123 . \\
2\end{array}$ & 9.4 & 1175.8 & 9.8 & $\begin{array}{r}1273 . \\
9\end{array}$ & 21.1 & 1273.9 & 21.1 & 88.2 \\
\hline $\begin{array}{l}\text { BU08135- } \\
4\end{array}$ & $\begin{array}{r}135 \\
5 \\
\end{array}$ & 82276 & 14.6 & $\begin{array}{r}12.00 \\
08 \\
\end{array}$ & 0.9 & 1.7778 & 2.0 & $\begin{array}{r}0.154 \\
7 \\
\end{array}$ & $\begin{array}{r}1 . \\
8\end{array}$ & $\begin{array}{r}0.8 \\
9 \\
\end{array}$ & 927.4 & 15.5 & 1037.4 & 13.0 & $\begin{array}{r}1276 . \\
9 \\
\end{array}$ & 17.5 & 1276.9 & 17.5 & 72.6 \\
\hline $\begin{array}{l}\text { BU08135- } \\
24\end{array}$ & 732 & 61404 & 6.7 & $\begin{array}{r}11.76 \\
59 \\
\end{array}$ & 1.1 & 2.5143 & 1.2 & $\begin{array}{r}0.214 \\
6 \\
\end{array}$ & $\begin{array}{r}0 . \\
6 \\
\end{array}$ & $\begin{array}{r}0.5 \\
1 \\
\end{array}$ & $\begin{array}{r}1253 . \\
0 \\
\end{array}$ & 7.2 & 1276.2 & 8.9 & $\begin{array}{r}1315 . \\
3 \\
\end{array}$ & 20.4 & 1315.3 & 20.4 & 95.3 \\
\hline BU08135- & 341 & 28886 & 1.2 & 11.55 & 1.5 & 2.5698 & 1.6 & 0.215 & 0. & 0.3 & 1256. & 5.7 & 1292.1 & 11.5 & 1351. & 28.8 & 1351.1 & 28.8 & 93.0 \\
\hline
\end{tabular}




\begin{tabular}{|c|c|c|c|c|c|c|c|c|c|c|c|c|c|c|c|c|c|c|c|}
\hline 54 & & & & 02 & & & & 3 & 5 & 2 & 8 & & & & 1 & & & & \\
\hline $\begin{array}{l}\text { BU08135- } \\
103\end{array}$ & 414 & 17344 & 9.0 & $\begin{array}{r}11.49 \\
89 \\
\end{array}$ & 1.7 & 2.7094 & 1.8 & $\begin{array}{r}0.226 \\
0 \\
\end{array}$ & $\begin{array}{r}0 . \\
6 \\
\end{array}$ & $\begin{array}{r}0.3 \\
2 \\
\end{array}$ & $\begin{array}{r}1313 . \\
3 \\
\end{array}$ & 6.7 & 1331.0 & 13.1 & $\begin{array}{r}1359 . \\
7 \\
\end{array}$ & 32.4 & 1359.7 & 32.4 & 96.6 \\
\hline $\begin{array}{l}\text { BU08135- } \\
29\end{array}$ & 243 & 24084 & 1.3 & $\begin{array}{r}11.46 \\
63\end{array}$ & 1.7 & 2.7673 & 4.4 & $\begin{array}{r}0.230 \\
1\end{array}$ & $\begin{array}{r}4 . \\
1\end{array}$ & $\begin{array}{r}0.9 \\
3\end{array}$ & $\begin{array}{r}1335 . \\
2\end{array}$ & 49.0 & 1346.8 & 32.7 & $\begin{array}{r}1365 . \\
2\end{array}$ & 31.8 & 1365.2 & 31.8 & 97.8 \\
\hline $\begin{array}{l}\text { BU08135- } \\
22\end{array}$ & 373 & 16502 & 1.5 & $\begin{array}{r}11.43 \\
53\end{array}$ & 2.0 & 2.4817 & 3.6 & $\begin{array}{r}0.205 \\
8\end{array}$ & $\begin{array}{r}3 . \\
0\end{array}$ & $\begin{array}{r}0.8 \\
4\end{array}$ & $\begin{array}{r}1206 . \\
5\end{array}$ & 33.0 & 1266.7 & 26.0 & $\begin{array}{r}1370 . \\
4\end{array}$ & 38.0 & 1370.4 & 38.0 & 88.0 \\
\hline $\begin{array}{l}\text { BU08135- } \\
47\end{array}$ & $\begin{array}{r}130 \\
1 \\
\end{array}$ & 58912 & 3.2 & $\begin{array}{r}11.14 \\
60\end{array}$ & 1.1 & 2.4962 & 4.3 & $\begin{array}{r}0.201 \\
8\end{array}$ & $\begin{array}{r}4 . \\
2\end{array}$ & $\begin{array}{r}0.9 \\
7\end{array}$ & $\begin{array}{r}1184 . \\
9\end{array}$ & 45.4 & 1270.9 & 31.4 & $\begin{array}{r}1419 . \\
5\end{array}$ & 20.6 & 1419.5 & 20.6 & 83.5 \\
\hline $\begin{array}{l}\text { BU08135- } \\
87\end{array}$ & $\begin{array}{r}196 \\
8 \\
\end{array}$ & $\begin{array}{r}13538 \\
4 \\
\end{array}$ & 4.3 & $\begin{array}{r}11.01 \\
76 \\
\end{array}$ & 0.7 & 2.8024 & 1.5 & $\begin{array}{r}0.223 \\
9 \\
\end{array}$ & $\begin{array}{r}1 . \\
3\end{array}$ & $\begin{array}{r}0.8 \\
8 \\
\end{array}$ & $\begin{array}{r}1302 . \\
6 \\
\end{array}$ & 15.6 & 1356.2 & 11.2 & $\begin{array}{r}1441 . \\
6 \\
\end{array}$ & 13.3 & 1441.6 & 13.3 & 90.4 \\
\hline $\begin{array}{l}\text { BU08135- } \\
33\end{array}$ & 212 & 21152 & 0.7 & $\begin{array}{r}10.95 \\
97\end{array}$ & 1.8 & 2.9340 & 2.8 & $\begin{array}{r}0.233 \\
2\end{array}$ & $\begin{array}{r}2 . \\
2\end{array}$ & $\begin{array}{r}0.7 \\
8 \\
\end{array}$ & $\begin{array}{r}1351 . \\
3 \\
\end{array}$ & 26.9 & 1390.7 & 21.4 & $\begin{array}{r}1451 . \\
7 \\
\end{array}$ & 33.7 & 1451.7 & 33.7 & 93.1 \\
\hline $\begin{array}{l}\text { BU08135- } \\
102\end{array}$ & 226 & 25170 & 0.9 & $\begin{array}{r}10.94 \\
83\end{array}$ & 1.3 & 3.1731 & 1.7 & $\begin{array}{r}0.252 \\
0\end{array}$ & $\begin{array}{r}1 . \\
0\end{array}$ & $\begin{array}{r}0.6 \\
2\end{array}$ & $\begin{array}{r}1448 . \\
6\end{array}$ & 13.4 & 1450.6 & 12.8 & $\begin{array}{r}1453 . \\
7\end{array}$ & 24.6 & 1453.7 & 24.6 & 99.6 \\
\hline $\begin{array}{l}\text { BU08135- } \\
14\end{array}$ & 976 & 84610 & 1.6 & $\begin{array}{r}10.64 \\
11 \\
\end{array}$ & 2.0 & 3.0363 & 2.5 & $\begin{array}{r}0.234 \\
3 \\
\end{array}$ & $\begin{array}{r}1 . \\
5\end{array}$ & $\begin{array}{r}0.6 \\
1 \\
\end{array}$ & $\begin{array}{r}1357 . \\
2\end{array}$ & 18.5 & 1416.8 & 19.0 & $\begin{array}{r}1507 . \\
6 \\
\end{array}$ & 37.2 & 1507.6 & 37.2 & 90.0 \\
\hline $\begin{array}{l}\text { BU08135- } \\
65\end{array}$ & 370 & 34340 & 1.5 & $\begin{array}{r}9.946 \\
1\end{array}$ & 1.0 & 3.7647 & 1.1 & $\begin{array}{r}0.271 \\
6\end{array}$ & $\begin{array}{l}0 . \\
6\end{array}$ & $\begin{array}{r}0.5 \\
0\end{array}$ & $\begin{array}{r}1548 . \\
8\end{array}$ & 7.7 & 1585.3 & 8.9 & $\begin{array}{r}1634 . \\
1\end{array}$ & 17.8 & 1634.1 & 17.8 & 94.8 \\
\hline $\begin{array}{l}\text { BU08135- } \\
85\end{array}$ & 368 & 41040 & 2.2 & $\begin{array}{r}9.804 \\
6 \\
\end{array}$ & 1.3 & 3.8730 & 1.5 & $\begin{array}{r}0.275 \\
4 \\
\end{array}$ & $\begin{array}{r}0 . \\
6\end{array}$ & $\begin{array}{r}0.4 \\
2\end{array}$ & $\begin{array}{r}1568 . \\
2\end{array}$ & 8.6 & 1608.1 & 11.9 & $\begin{array}{r}1660 . \\
7\end{array}$ & 24.8 & 1660.7 & 24.8 & 94.4 \\
\hline $\begin{array}{l}\text { BU08135- } \\
42\end{array}$ & 380 & 34816 & 4.9 & $\begin{array}{r}9.374 \\
1\end{array}$ & 2.4 & 3.9874 & 2.5 & $\begin{array}{r}0.271 \\
1\end{array}$ & $\begin{array}{l}0 . \\
7\end{array}$ & $\begin{array}{r}0.2 \\
7\end{array}$ & $\begin{array}{r}1546 . \\
3\end{array}$ & 9.3 & 1631.6 & 20.5 & $\begin{array}{r}1743 . \\
4\end{array}$ & 44.6 & 1743.4 & 44.6 & 88.7 \\
\hline $\begin{array}{l}\text { BU08135- } \\
83\end{array}$ & 428 & 43552 & 2.1 & $\begin{array}{r}8.085 \\
6\end{array}$ & 0.7 & 5.7728 & 1.5 & $\begin{array}{r}0.338 \\
5\end{array}$ & $\begin{array}{r}1 . \\
3\end{array}$ & $\begin{array}{r}0.8 \\
6\end{array}$ & $\begin{array}{r}1879 . \\
6\end{array}$ & 20.5 & 1942.3 & 12.6 & $\begin{array}{r}2009 . \\
9\end{array}$ & 13.1 & 2009.9 & 13.1 & 93.5 \\
\hline $\begin{array}{l}\text { BU08135- } \\
30\end{array}$ & 564 & 82268 & 12.7 & $\begin{array}{r}7.699 \\
3\end{array}$ & 1.4 & 6.4318 & 1.4 & $\begin{array}{r}0.359 \\
2\end{array}$ & $\begin{array}{r}0 . \\
5\end{array}$ & $\begin{array}{r}0.3 \\
5\end{array}$ & $\begin{array}{r}1978 . \\
2\end{array}$ & 8.5 & 2036.6 & 12.7 & $\begin{array}{r}2096 . \\
3\end{array}$ & 23.9 & 2096.3 & 23.9 & 94.4 \\
\hline $\begin{array}{l}\text { BU08135- } \\
3\end{array}$ & 808 & 53740 & 6.1 & $\begin{array}{r}6.886 \\
4 \\
\end{array}$ & 1.4 & 5.6949 & 5.8 & $\begin{array}{r}0.284 \\
4 \\
\end{array}$ & $\begin{array}{r}5 . \\
6 \\
\end{array}$ & $\begin{array}{r}0.9 \\
7 \\
\end{array}$ & $\begin{array}{r}1613 . \\
6 \\
\end{array}$ & 80.4 & 1930.6 & 50.2 & $\begin{array}{r}2290 . \\
3 \\
\end{array}$ & 24.4 & 2290.3 & 24.4 & 70.5 \\
\hline $\begin{array}{l}\text { BU08135- } \\
64\end{array}$ & 188 & 25590 & 3.0 & $\begin{array}{r}6.447 \\
6\end{array}$ & 0.9 & 7.9346 & 6.0 & $\begin{array}{r}0.371 \\
0\end{array}$ & $\begin{array}{r}5 . \\
9\end{array}$ & $\begin{array}{r}0.9 \\
9\end{array}$ & $\begin{array}{r}2034 . \\
3\end{array}$ & $\begin{array}{r}103 . \\
6 \\
\end{array}$ & 2223.6 & 54.2 & $\begin{array}{r}2402 . \\
9\end{array}$ & 14.8 & 2402.9 & 14.8 & 84.7 \\
\hline $\begin{array}{l}\text { BU08135- } \\
105\end{array}$ & 135 & 26160 & 1.7 & $\begin{array}{r}6.386 \\
8\end{array}$ & 2.1 & 9.9858 & 2.3 & $\begin{array}{r}0.462 \\
6\end{array}$ & $\begin{array}{c}0 . \\
9\end{array}$ & $\begin{array}{r}0.4 \\
0\end{array}$ & $\begin{array}{r}2450 . \\
8\end{array}$ & 18.3 & 2433.5 & 21.0 & $\begin{array}{r}2419 . \\
0\end{array}$ & 35.5 & 2419.0 & 35.5 & $\begin{array}{r}101 . \\
3\end{array}$ \\
\hline $\begin{array}{l}\text { BU08135- } \\
78\end{array}$ & 790 & 85728 & 4.7 & $\begin{array}{r}6.345 \\
6\end{array}$ & 0.9 & 8.1294 & 3.6 & $\begin{array}{r}0.374 \\
1\end{array}$ & $\begin{array}{r}3 . \\
5\end{array}$ & $\begin{array}{r}0.9 \\
7\end{array}$ & $\begin{array}{r}2048 . \\
8\end{array}$ & 61.6 & 2245.5 & 32.8 & $\begin{array}{r}2430 . \\
0\end{array}$ & 15.1 & 2430.0 & 15.1 & 84.3 \\
\hline $\begin{array}{l}\text { BU08135- } \\
98\end{array}$ & 460 & 71690 & 6.2 & $\begin{array}{r}6.311 \\
9\end{array}$ & 1.3 & 9.7534 & 1.7 & $\begin{array}{r}0.446 \\
5\end{array}$ & $\begin{array}{r}1 . \\
2\end{array}$ & $\begin{array}{r}0.6 \\
9\end{array}$ & $\begin{array}{r}2379 . \\
6\end{array}$ & 23.7 & 2411.8 & 16.0 & $\begin{array}{r}2439 . \\
0\end{array}$ & 21.3 & 2439.0 & 21.3 & 97.6 \\
\hline $\begin{array}{l}\text { BU08135- } \\
59\end{array}$ & $\begin{array}{r}156 \\
6 \\
\end{array}$ & $\begin{array}{r}20310 \\
6 \\
\end{array}$ & 10.6 & $\begin{array}{r}6.229 \\
8 \\
\end{array}$ & 0.8 & 9.3484 & 1.2 & $\begin{array}{r}0.422 \\
4 \\
\end{array}$ & $\begin{array}{c}0 . \\
9\end{array}$ & $\begin{array}{r}0.7 \\
4 \\
\end{array}$ & $\begin{array}{r}2271 . \\
3 \\
\end{array}$ & 17.4 & 2372.8 & 11.2 & $\begin{array}{r}2461 . \\
1\end{array}$ & 13.9 & 2461.1 & 13.9 & 92.3 \\
\hline $\begin{array}{l}\text { BU08135- } \\
60\end{array}$ & $\begin{array}{r}138 \\
0 \\
\end{array}$ & $\begin{array}{r}16982 \\
8\end{array}$ & 1.8 & $\begin{array}{r}6.112 \\
5\end{array}$ & 1.1 & 9.8249 & 1.2 & $\begin{array}{r}0.435 \\
6\end{array}$ & $\begin{array}{c}0 . \\
6\end{array}$ & $\begin{array}{r}0.4 \\
9\end{array}$ & $\begin{array}{r}2330 . \\
7\end{array}$ & 11.7 & 2418.5 & 11.4 & $\begin{array}{r}2493 . \\
2\end{array}$ & 18.2 & 2493.2 & 18.2 & 93.5 \\
\hline $\begin{array}{l}\text { BU08135- } \\
94\end{array}$ & 128 & 19846 & 1.4 & $\begin{array}{r}6.073 \\
1\end{array}$ & 0.8 & $\begin{array}{r}10.320 \\
2\end{array}$ & 1.1 & $\begin{array}{r}0.454 \\
6\end{array}$ & $\begin{array}{r}0 . \\
8\end{array}$ & $\begin{array}{r}0.7 \\
0\end{array}$ & $\begin{array}{r}2415 . \\
5\end{array}$ & 16.1 & 2463.9 & 10.5 & $\begin{array}{r}2504 . \\
1\end{array}$ & 13.6 & 2504.1 & 13.6 & 96.5 \\
\hline $\begin{array}{l}\text { BU08135- } \\
27\end{array}$ & 229 & 40786 & 4.5 & $\begin{array}{r}5.816 \\
5\end{array}$ & 1.5 & $\begin{array}{r}10.728 \\
4\end{array}$ & 2.0 & $\begin{array}{r}0.452 \\
6\end{array}$ & $\begin{array}{l}1 . \\
3\end{array}$ & $\begin{array}{r}0.6 \\
4\end{array}$ & $\begin{array}{r}2406 . \\
7\end{array}$ & 25.1 & 2499.9 & 18.3 & $\begin{array}{r}2576 . \\
5\end{array}$ & 25.4 & 2576.5 & 25.4 & 93.4 \\
\hline $\begin{array}{l}\text { BU08135- } \\
6\end{array}$ & 79 & 13492 & 2.4 & $\begin{array}{r}4.896 \\
7\end{array}$ & 1.1 & $\begin{array}{r}12.869 \\
0\end{array}$ & 2.9 & $\begin{array}{r}0.457 \\
0\end{array}$ & $\begin{array}{r}2 . \\
7\end{array}$ & $\begin{array}{r}0.9 \\
2\end{array}$ & $\begin{array}{r}2426 . \\
4\end{array}$ & 53.8 & 2670.1 & 27.3 & $\begin{array}{r}2860 . \\
2\end{array}$ & 18.6 & 2860.2 & 18.6 & 84.8 \\
\hline $\begin{array}{l}\text { BU08135- } \\
80\end{array}$ & 107 & 21512 & 1.7 & $\begin{array}{r}4.883 \\
2\end{array}$ & 0.8 & $\begin{array}{r}14.684 \\
1\end{array}$ & 1.0 & $\begin{array}{r}0.520 \\
1\end{array}$ & $\begin{array}{r}0 . \\
6\end{array}$ & $\begin{array}{r}0.6 \\
1\end{array}$ & $\begin{array}{r}2699 . \\
4\end{array}$ & 13.7 & 2795.0 & 9.7 & $\begin{array}{r}2864 . \\
7\end{array}$ & 13.2 & 2864.7 & 13.2 & 94.2 \\
\hline
\end{tabular}




\begin{tabular}{|c|c|c|c|c|c|c|c|c|c|c|c|c|c|c|c|c|c|c|c|}
\hline NBH3-18 & 371 & 61130 & 0.9 & $\begin{array}{r}14.18 \\
89 \\
\end{array}$ & 2.8 & 1.4263 & 4.0 & $\begin{array}{r}0.146 \\
8 \\
\end{array}$ & $\begin{array}{r}2 . \\
8 \\
\end{array}$ & $\begin{array}{r}0.7 \\
1 \\
\end{array}$ & 882.9 & 23.3 & 900.0 & 23.7 & 942.3 & 57.2 & 882.9 & 23.3 & 93.7 \\
\hline NBH3-41 & $\begin{array}{r}117 \\
0\end{array}$ & $\begin{array}{r}18726 \\
0\end{array}$ & 1.5 & $\begin{array}{r}14.36 \\
04\end{array}$ & 1.2 & 1.4581 & 2.6 & $\begin{array}{r}0.151 \\
9\end{array}$ & $\begin{array}{r}2 . \\
3\end{array}$ & $\begin{array}{r}0.8 \\
8\end{array}$ & 911.4 & 19.4 & 913.2 & 15.6 & 917.6 & 25.3 & 911.4 & 19.4 & 99.3 \\
\hline NBH3-46 & $\begin{array}{r}122 \\
0\end{array}$ & $\begin{array}{r}15041 \\
5\end{array}$ & 1.6 & $\begin{array}{r}14.03 \\
58\end{array}$ & 1.0 & 1.4968 & 1.4 & $\begin{array}{r}0.152 \\
4\end{array}$ & $\begin{array}{l}1 . \\
0\end{array}$ & $\begin{array}{r}0.7 \\
1\end{array}$ & 914.2 & 8.5 & 929.1 & 8.6 & 964.5 & 20.4 & 914.2 & 8.5 & 94.8 \\
\hline NBH3-89 & 124 & 33380 & 0.4 & $\begin{array}{r}14.23 \\
16\end{array}$ & 2.5 & 1.5016 & 3.9 & $\begin{array}{r}0.155 \\
0\end{array}$ & $\begin{array}{r}3 . \\
0\end{array}$ & $\begin{array}{r}0.7 \\
6\end{array}$ & 928.9 & 25.9 & 931.0 & 23.9 & 936.1 & 51.9 & 928.9 & 25.9 & 99.2 \\
\hline NBH3-36 & 391 & 57310 & 2.1 & $\begin{array}{r}13.92 \\
50 \\
\end{array}$ & 1.0 & 1.5391 & 1.4 & $\begin{array}{r}0.155 \\
4 \\
\end{array}$ & $\begin{array}{r}1 . \\
0\end{array}$ & $\begin{array}{r}0.6 \\
9 \\
\end{array}$ & 931.4 & 8.7 & 946.1 & 8.9 & 980.6 & 21.2 & 931.4 & 8.7 & 95.0 \\
\hline NBH3-31 & 264 & 41220 & 2.4 & $\begin{array}{r}13.61 \\
62\end{array}$ & 2.2 & 1.6281 & 2.6 & $\begin{array}{r}0.160 \\
8\end{array}$ & $\begin{array}{r}1 . \\
4\end{array}$ & $\begin{array}{r}0.5 \\
3\end{array}$ & 961.1 & 12.5 & 981.1 & 16.5 & $\begin{array}{r}1026 . \\
2\end{array}$ & 44.7 & 961.1 & 12.5 & 93.7 \\
\hline NBH3-6 & 127 & 29625 & 1.3 & $\begin{array}{r}13.53 \\
46\end{array}$ & 3.6 & 1.7366 & 3.8 & $\begin{array}{r}0.170 \\
5\end{array}$ & $\begin{array}{l}1 . \\
0\end{array}$ & $\begin{array}{r}0.2 \\
7\end{array}$ & $\begin{array}{r}1014 . \\
7\end{array}$ & 9.4 & 1022.2 & 24.3 & $\begin{array}{r}1038 . \\
3\end{array}$ & 73.3 & 1038.3 & 73.3 & 97.7 \\
\hline NBH3-45 & 779 & $\begin{array}{r}11274 \\
0 \\
\end{array}$ & 1.8 & $\begin{array}{r}13.42 \\
01 \\
\end{array}$ & 1.6 & 1.6951 & 2.2 & $\begin{array}{r}0.165 \\
0 \\
\end{array}$ & $\begin{array}{l}1 . \\
5\end{array}$ & $\begin{array}{r}0.6 \\
7 \\
\end{array}$ & 984.5 & 13.2 & 1006.7 & 13.7 & $\begin{array}{r}1055 . \\
4\end{array}$ & 32.0 & 1055.4 & 32.0 & 93.3 \\
\hline NBH3-37 & 492 & 59235 & 1.5 & $\begin{array}{r}13.13 \\
99 \\
\end{array}$ & 1.4 & 1.7814 & 1.8 & $\begin{array}{r}0.169 \\
8 \\
\end{array}$ & $\begin{array}{c}1 . \\
1\end{array}$ & $\begin{array}{r}0.6 \\
1 \\
\end{array}$ & $\begin{array}{r}1010 . \\
8 \\
\end{array}$ & 10.1 & 1038.7 & 11.5 & $\begin{array}{r}1097 . \\
8 \\
\end{array}$ & 28.0 & 1097.8 & 28.0 & 92.1 \\
\hline NBH3-17 & 574 & $\begin{array}{r}10122 \\
0 \\
\end{array}$ & 0.7 & $\begin{array}{r}12.93 \\
28 \\
\end{array}$ & 2.6 & 2.0309 & 2.7 & $\begin{array}{r}0.190 \\
5 \\
\end{array}$ & $\begin{array}{r}1 . \\
0\end{array}$ & $\begin{array}{r}0.3 \\
7 \\
\end{array}$ & $\begin{array}{r}1124 . \\
0\end{array}$ & 10.3 & 1125.9 & 18.6 & $\begin{array}{r}1129 . \\
5\end{array}$ & 50.8 & 1129.5 & 50.8 & 99.5 \\
\hline NBH3-75 & 217 & 52630 & 1.2 & $\begin{array}{r}12.88 \\
39\end{array}$ & 2.6 & 2.0462 & 3.1 & $\begin{array}{r}0.191 \\
2\end{array}$ & $\begin{array}{l}1 . \\
7\end{array}$ & $\begin{array}{r}0.5 \\
6\end{array}$ & $\begin{array}{r}1127 . \\
9\end{array}$ & 17.8 & 1131.0 & 21.0 & $\begin{array}{r}1137 . \\
0\end{array}$ & 50.8 & 1137.0 & 50.8 & 99.2 \\
\hline NBH3-63 & 960 & $\begin{array}{r}22505 \\
0\end{array}$ & 2.2 & $\begin{array}{r}12.87 \\
75\end{array}$ & 2.1 & 2.0638 & 2.3 & $\begin{array}{r}0.192 \\
7\end{array}$ & $\begin{array}{l}1 . \\
0\end{array}$ & $\begin{array}{r}0.4 \\
3\end{array}$ & $\begin{array}{r}1136 . \\
2\end{array}$ & 10.4 & 1136.9 & 16.0 & $\begin{array}{r}1138 . \\
0\end{array}$ & 42.0 & 1138.0 & 42.0 & 99.8 \\
\hline NBH3-21 & 197 & 28695 & 1.2 & $\begin{array}{r}12.87 \\
02\end{array}$ & 3.5 & 1.8237 & 4.5 & $\begin{array}{r}0.170 \\
2\end{array}$ & $\begin{array}{l}2 . \\
7\end{array}$ & $\begin{array}{r}0.6 \\
1\end{array}$ & $\begin{array}{r}1013 . \\
4\end{array}$ & 25.5 & 1054.0 & 29.2 & $\begin{array}{r}1139 . \\
2\end{array}$ & 70.2 & 1139.2 & 70.2 & 89.0 \\
\hline NBH3-5 & 184 & 62295 & 1.9 & $\begin{array}{r}12.66 \\
34 \\
\end{array}$ & 4.4 & 2.2500 & 4.6 & $\begin{array}{r}0.206 \\
6 \\
\end{array}$ & $\begin{array}{r}1 . \\
5\end{array}$ & $\begin{array}{r}0.3 \\
2 \\
\end{array}$ & $\begin{array}{r}1210 . \\
9\end{array}$ & 16.2 & 1196.8 & 32.5 & $\begin{array}{r}1171 . \\
3\end{array}$ & 86.8 & 1171.3 & 86.8 & $\begin{array}{r}103 . \\
4 \\
\end{array}$ \\
\hline NBH3-64 & 128 & 83905 & 1.3 & $\begin{array}{r}12.57 \\
00\end{array}$ & 3.6 & 2.1954 & 3.7 & $\begin{array}{r}0.200 \\
1\end{array}$ & $\begin{array}{r}1 . \\
0\end{array}$ & $\begin{array}{r}0.2 \\
7\end{array}$ & $\begin{array}{r}1176 . \\
1\end{array}$ & 10.8 & 1179.6 & 26.1 & $\begin{array}{r}1185 . \\
9\end{array}$ & 71.4 & 1185.9 & 71.4 & 99.2 \\
\hline NBH3-72 & 157 & $\begin{array}{r}11080 \\
0\end{array}$ & 1.6 & $\begin{array}{r}12.56 \\
27\end{array}$ & 2.1 & 2.1169 & 2.8 & $\begin{array}{r}0.192 \\
9\end{array}$ & $\begin{array}{r}1 . \\
8\end{array}$ & $\begin{array}{r}0.6 \\
5\end{array}$ & $\begin{array}{r}1136 . \\
9\end{array}$ & 19.0 & 1154.3 & 19.3 & $\begin{array}{r}1187 . \\
1\end{array}$ & 42.1 & 1187.1 & 42.1 & 95.8 \\
\hline NBH3-79 & 496 & 36795 & 1.4 & $\begin{array}{r}12.53 \\
42\end{array}$ & 3.9 & 2.1768 & 4.1 & $\begin{array}{r}0.197 \\
9\end{array}$ & $\begin{array}{r}1 . \\
4\end{array}$ & $\begin{array}{r}0.3 \\
4\end{array}$ & $\begin{array}{r}1164 . \\
0\end{array}$ & 14.6 & 1173.7 & 28.4 & $\begin{array}{r}1191 . \\
6\end{array}$ & 76.0 & 1191.6 & 76.0 & 97.7 \\
\hline NBH3-80 & 144 & 46155 & 1.1 & $\begin{array}{r}12.37 \\
33 \\
\end{array}$ & 2.3 & 2.2664 & 2.6 & $\begin{array}{r}0.203 \\
4 \\
\end{array}$ & $\begin{array}{r}1 . \\
1\end{array}$ & $\begin{array}{r}0.4 \\
2 \\
\end{array}$ & $\begin{array}{r}1193 . \\
5 \\
\end{array}$ & 11.9 & 1201.9 & 18.2 & $\begin{array}{r}1217 . \\
0 \\
\end{array}$ & 46.1 & 1217.0 & 46.1 & 98.1 \\
\hline NBH3-57 & 604 & 72330 & 3.9 & $\begin{array}{r}12.29 \\
93 \\
\end{array}$ & 1.6 & 2.3122 & 2.8 & $\begin{array}{r}0.206 \\
3 \\
\end{array}$ & $\begin{array}{r}2 . \\
3 \\
\end{array}$ & $\begin{array}{r}0.8 \\
2 \\
\end{array}$ & $\begin{array}{r}1208 . \\
9\end{array}$ & 25.2 & 1216.0 & 19.8 & $\begin{array}{r}1228 . \\
8 \\
\end{array}$ & 31.2 & 1228.8 & 31.2 & 98.4 \\
\hline NBH3-4 & 528 & $\begin{array}{r}13269 \\
0\end{array}$ & 4.2 & $\begin{array}{r}11.70 \\
53\end{array}$ & 4.1 & 2.7929 & 4.3 & $\begin{array}{r}0.237 \\
1\end{array}$ & $\begin{array}{r}1 . \\
0\end{array}$ & $\begin{array}{r}0.2 \\
3 \\
\end{array}$ & $\begin{array}{r}1371 . \\
6\end{array}$ & 12.4 & 1353.6 & 31.9 & $\begin{array}{r}1325 . \\
3\end{array}$ & 80.2 & 1325.3 & 80.2 & $\begin{array}{r}103 . \\
5 \\
\end{array}$ \\
\hline NBH3-100 & 424 & $\begin{array}{r}14501 \\
5\end{array}$ & 1.6 & $\begin{array}{r}11.68 \\
25\end{array}$ & 3.2 & 2.7606 & 3.5 & $\begin{array}{r}0.233 \\
9\end{array}$ & $\begin{array}{l}1 . \\
3\end{array}$ & $\begin{array}{r}0.3 \\
7\end{array}$ & $\begin{array}{r}1354 . \\
9\end{array}$ & 15.8 & 1345.0 & 25.7 & $\begin{array}{r}1329 . \\
1\end{array}$ & 61.9 & 1329.1 & 61.9 & $\begin{array}{r}101 . \\
9\end{array}$ \\
\hline NBH3-54 & 319 & 50150 & 1.8 & $\begin{array}{r}11.62 \\
76 \\
\end{array}$ & 1.6 & 2.4105 & 3.0 & $\begin{array}{r}0.203 \\
3 \\
\end{array}$ & $\begin{array}{r}2 . \\
5 \\
\end{array}$ & $\begin{array}{r}0.8 \\
4 \\
\end{array}$ & $\begin{array}{r}1192 . \\
9 \\
\end{array}$ & 27.1 & 1245.7 & 21.2 & $\begin{array}{r}1338 . \\
2 \\
\end{array}$ & 30.7 & 1338.2 & 30.7 & 89.1 \\
\hline NBH3-51 & $\begin{array}{r}224 \\
5 \\
\end{array}$ & $\begin{array}{r}43420 \\
5 \\
\end{array}$ & 1.3 & $\begin{array}{r}11.59 \\
78 \\
\end{array}$ & 1.6 & 2.7148 & 1.9 & $\begin{array}{r}0.228 \\
4 \\
\end{array}$ & $\begin{array}{r}1 . \\
0 \\
\end{array}$ & $\begin{array}{r}0.5 \\
4 \\
\end{array}$ & $\begin{array}{r}1325 . \\
9 \\
\end{array}$ & 12.0 & 1332.5 & 13.8 & $\begin{array}{r}1343 . \\
2 \\
\end{array}$ & 30.1 & 1343.2 & 30.1 & 98.7 \\
\hline NBH3-73 & 901 & $\begin{array}{r}27610 \\
5\end{array}$ & 1.1 & $\begin{array}{r}11.56 \\
45\end{array}$ & 1.5 & 2.7531 & 1.8 & $\begin{array}{r}0.230 \\
9\end{array}$ & $\begin{array}{r}1 . \\
0\end{array}$ & $\begin{array}{r}0.5 \\
5\end{array}$ & $\begin{array}{r}1339 . \\
3\end{array}$ & 12.1 & 1342.9 & 13.6 & $\begin{array}{r}1348 . \\
7\end{array}$ & 29.5 & 1348.7 & 29.5 & 99.3 \\
\hline
\end{tabular}




\begin{tabular}{|c|c|c|c|c|c|c|c|c|c|c|c|c|c|c|c|c|c|c|c|}
\hline NBH3-39 & $\begin{array}{r}163 \\
1\end{array}$ & $\begin{array}{r}14899 \\
0\end{array}$ & 2.7 & $\begin{array}{r}11.46 \\
66\end{array}$ & 1.4 & 2.6921 & 1.7 & $\begin{array}{r}0.223 \\
9\end{array}$ & $\begin{array}{r}1 . \\
0\end{array}$ & $\begin{array}{r}0.5 \\
7\end{array}$ & $\begin{array}{r}1302 . \\
4\end{array}$ & 11.8 & 1326.3 & 12.9 & $\begin{array}{r}1365 . \\
1\end{array}$ & 27.5 & 1365.1 & 27.5 & 95.4 \\
\hline NBH3-13 & $\begin{array}{r}142 \\
3\end{array}$ & $\begin{array}{r}21287 \\
5\end{array}$ & 1.8 & $\begin{array}{r}11.40 \\
26\end{array}$ & 2.2 & 2.7436 & 2.4 & $\begin{array}{r}0.226 \\
9\end{array}$ & $\begin{array}{r}1 . \\
0\end{array}$ & $\begin{array}{r}0.4 \\
2\end{array}$ & $\begin{array}{r}1318 . \\
2\end{array}$ & 11.9 & 1340.4 & 17.7 & $\begin{array}{r}1375 . \\
9\end{array}$ & 41.5 & 1375.9 & 41.5 & 95.8 \\
\hline NBH3-23 & 366 & 32040 & 1.4 & $\begin{array}{r}11.27 \\
37\end{array}$ & 2.1 & 2.5055 & 2.3 & $\begin{array}{r}0.204 \\
9\end{array}$ & $\begin{array}{r}1 . \\
0\end{array}$ & $\begin{array}{r}0.4 \\
3\end{array}$ & $\begin{array}{r}1201 . \\
4\end{array}$ & 11.0 & 1273.6 & 17.0 & $\begin{array}{r}1397 . \\
7\end{array}$ & 40.7 & 1397.7 & 40.7 & 86.0 \\
\hline NBH3-26 & 50 & 13205 & 1.0 & $\begin{array}{r}11.12 \\
17\end{array}$ & 4.0 & 2.8484 & 4.1 & $\begin{array}{r}0.229 \\
8\end{array}$ & $\begin{array}{r}1 . \\
0\end{array}$ & $\begin{array}{r}0.2 \\
4\end{array}$ & $\begin{array}{r}1333 . \\
3\end{array}$ & 12.0 & 1368.4 & 31.2 & $\begin{array}{r}1423 . \\
7\end{array}$ & 76.9 & 1423.7 & 76.9 & 93.6 \\
\hline NBH3-29 & 158 & 30675 & 1.3 & $\begin{array}{r}11.09 \\
28 \\
\end{array}$ & 1.6 & 2.8480 & 2.1 & $\begin{array}{r}0.229 \\
1 \\
\end{array}$ & $\begin{array}{r}1 . \\
4\end{array}$ & $\begin{array}{r}0.6 \\
5\end{array}$ & $\begin{array}{r}1329 . \\
9\end{array}$ & 16.3 & 1368.3 & 15.7 & $\begin{array}{r}1428 . \\
7\end{array}$ & 30.2 & 1428.7 & 30.2 & 93.1 \\
\hline NBH3-55 & 632 & 24305 & 1.5 & $\begin{array}{r}10.79 \\
00 \\
\end{array}$ & 4.1 & 2.7116 & 4.8 & $\begin{array}{r}0.212 \\
2 \\
\end{array}$ & $\begin{array}{r}2 . \\
5 \\
\end{array}$ & $\begin{array}{r}0.5 \\
1 \\
\end{array}$ & $\begin{array}{r}1240 . \\
5 \\
\end{array}$ & 28.0 & 1331.6 & 35.8 & $\begin{array}{r}1481 . \\
3 \\
\end{array}$ & 78.4 & 1481.3 & 78.4 & 83.7 \\
\hline NBH3-98 & 982 & 71115 & 1.8 & $\begin{array}{r}10.66 \\
33\end{array}$ & 2.9 & 2.5706 & 3.6 & $\begin{array}{r}0.198 \\
8\end{array}$ & $\begin{array}{r}2 . \\
1\end{array}$ & $\begin{array}{r}0.5 \\
9\end{array}$ & $\begin{array}{r}1168 . \\
9\end{array}$ & 22.7 & 1292.3 & 26.2 & $\begin{array}{r}1503 . \\
7\end{array}$ & 54.6 & 1503.7 & 54.6 & 77.7 \\
\hline NBH3-91 & 350 & 62445 & 0.8 & $\begin{array}{r}10.60 \\
59 \\
\end{array}$ & 1.0 & 3.2578 & 1.6 & $\begin{array}{r}0.250 \\
6\end{array}$ & $\begin{array}{r}1 . \\
2\end{array}$ & $\begin{array}{r}0.7 \\
7\end{array}$ & $\begin{array}{r}1441 . \\
6\end{array}$ & 15.4 & 1471.0 & 12.1 & $\begin{array}{r}1513 . \\
8\end{array}$ & 18.9 & 1513.8 & 18.9 & 95.2 \\
\hline NBH3-48 & 549 & $\begin{array}{r}10662 \\
5 \\
\end{array}$ & 1.6 & $\begin{array}{r}10.51 \\
05 \\
\end{array}$ & 1.4 & 3.3644 & 1.7 & $\begin{array}{r}0.256 \\
5 \\
\end{array}$ & $\begin{array}{r}1 . \\
0 \\
\end{array}$ & $\begin{array}{r}0.5 \\
9 \\
\end{array}$ & $\begin{array}{r}1471 . \\
7 \\
\end{array}$ & 13.2 & 1496.2 & 13.3 & $\begin{array}{r}1530 . \\
9 \\
\end{array}$ & 25.8 & 1530.9 & 25.8 & 96.1 \\
\hline NBH3-27 & 554 & $\begin{array}{r}10485 \\
0\end{array}$ & 1.3 & $\begin{array}{r}10.37 \\
58\end{array}$ & 1.3 & 3.4479 & 1.6 & $\begin{array}{r}0.259 \\
5\end{array}$ & $\begin{array}{r}1 . \\
0\end{array}$ & $\begin{array}{r}0.6 \\
2\end{array}$ & $\begin{array}{r}1487 . \\
1\end{array}$ & 13.3 & 1515.4 & 12.6 & $\begin{array}{r}1555 . \\
1\end{array}$ & 23.5 & 1555.1 & 23.5 & 95.6 \\
\hline NBH3-28 & 711 & 98625 & 4.1 & $\begin{array}{r}10.23 \\
29 \\
\end{array}$ & 2.6 & 3.1010 & 6.3 & $\begin{array}{r}0.230 \\
1 \\
\end{array}$ & $\begin{array}{r}5 . \\
7 \\
\end{array}$ & $\begin{array}{r}0.9 \\
1 \\
\end{array}$ & $\begin{array}{r}1335 . \\
2 \\
\end{array}$ & 68.9 & 1432.9 & 48.3 & $\begin{array}{r}1581 . \\
1 \\
\end{array}$ & 49.0 & 1581.1 & 49.0 & 84.4 \\
\hline NBH3-32 & 989 & $\begin{array}{r}21773 \\
0\end{array}$ & 1.3 & $\begin{array}{r}10.15 \\
63\end{array}$ & 2.2 & 3.5864 & 2.6 & $\begin{array}{r}0.264 \\
2\end{array}$ & $\begin{array}{r}1 . \\
4\end{array}$ & $\begin{array}{r}0.5 \\
4\end{array}$ & $\begin{array}{r}1511 . \\
2\end{array}$ & 19.1 & 1546.5 & 20.8 & $\begin{array}{r}1595 . \\
2\end{array}$ & 41.1 & 1595.2 & 41.1 & 94.7 \\
\hline NBH3-60 & 253 & 62510 & 1.7 & $\begin{array}{r}10.15 \\
48 \\
\end{array}$ & 2.0 & 3.5232 & 3.2 & $\begin{array}{r}0.259 \\
5 \\
\end{array}$ & $\begin{array}{r}2 . \\
5 \\
\end{array}$ & $\begin{array}{r}0.7 \\
7 \\
\end{array}$ & $\begin{array}{r}1487 . \\
2 \\
\end{array}$ & 32.7 & 1532.4 & 25.2 & $\begin{array}{r}1595 . \\
4 \\
\end{array}$ & 37.7 & 1595.4 & 37.7 & 93.2 \\
\hline NBH3-1 & 106 & 17545 & 1.1 & $\begin{array}{r}10.11 \\
23\end{array}$ & 3.0 & 3.1435 & 5.5 & $\begin{array}{r}0.230 \\
5\end{array}$ & $\begin{array}{r}4 . \\
5\end{array}$ & $\begin{array}{r}0.8 \\
3\end{array}$ & $\begin{array}{r}1337 . \\
4\end{array}$ & 54.7 & 1443.4 & 42.1 & $\begin{array}{r}1603 . \\
3\end{array}$ & 56.8 & 1603.3 & 56.8 & 83.4 \\
\hline NBH3-93 & 413 & $\begin{array}{r}10520 \\
5 \\
\end{array}$ & 0.9 & $\begin{array}{r}10.10 \\
11 \\
\end{array}$ & 2.4 & 3.6864 & 2.6 & $\begin{array}{r}0.270 \\
1 \\
\end{array}$ & $\begin{array}{r}1 . \\
0 \\
\end{array}$ & $\begin{array}{r}0.3 \\
9\end{array}$ & $\begin{array}{r}1541 . \\
1\end{array}$ & 14.3 & 1568.4 & 21.0 & $\begin{array}{r}1605 . \\
3\end{array}$ & 45.1 & 1605.3 & 45.1 & 96.0 \\
\hline NBH3-90 & 408 & $\begin{array}{r}17003 \\
0 \\
\end{array}$ & 1.6 & $\begin{array}{r}10.09 \\
61 \\
\end{array}$ & 2.3 & 3.8820 & 2.5 & $\begin{array}{r}0.284 \\
3 \\
\end{array}$ & $\begin{array}{r}1 . \\
0\end{array}$ & $\begin{array}{r}0.4 \\
0 \\
\end{array}$ & $\begin{array}{r}1612 . \\
8\end{array}$ & 14.3 & 1609.9 & 20.3 & $\begin{array}{r}1606 . \\
3\end{array}$ & 42.9 & 1606.3 & 42.9 & $\begin{array}{r}100 . \\
4\end{array}$ \\
\hline NBH3-52 & 283 & 66380 & 1.5 & $\begin{array}{r}10.07 \\
82 \\
\end{array}$ & 1.8 & 3.8029 & 2.0 & $\begin{array}{r}0.278 \\
0 \\
\end{array}$ & $\begin{array}{r}1 . \\
0 \\
\end{array}$ & $\begin{array}{r}0.4 \\
9 \\
\end{array}$ & $\begin{array}{r}1581 . \\
1 \\
\end{array}$ & 14.0 & 1593.4 & 16.4 & $\begin{array}{r}1609 . \\
6 \\
\end{array}$ & 33.2 & 1609.6 & 33.2 & 98.2 \\
\hline NBH3-44 & 114 & 22060 & 0.8 & $\begin{array}{r}10.06 \\
49 \\
\end{array}$ & 2.0 & 3.7338 & 3.1 & $\begin{array}{r}0.272 \\
6 \\
\end{array}$ & $\begin{array}{r}2 . \\
4\end{array}$ & $\begin{array}{r}0.7 \\
7 \\
\end{array}$ & $\begin{array}{r}1553 . \\
8\end{array}$ & 32.7 & 1578.7 & 24.6 & $\begin{array}{r}1612 . \\
0\end{array}$ & 36.5 & 1612.0 & 36.5 & 96.4 \\
\hline $\mathrm{NBH} 3-43$ & 91 & 44050 & 1.2 & $\begin{array}{r}10.06 \\
45 \\
\end{array}$ & 1.3 & 3.7715 & 2.3 & $\begin{array}{r}0.275 \\
3 \\
\end{array}$ & $\begin{array}{r}1 . \\
9 \\
\end{array}$ & $\begin{array}{r}0.8 \\
2 \\
\end{array}$ & $\begin{array}{r}1567 . \\
6 \\
\end{array}$ & 26.3 & 1586.7 & 18.6 & $\begin{array}{r}1612 . \\
1 \\
\end{array}$ & 25.0 & 1612.1 & 25.0 & 97.2 \\
\hline NBH3-95 & 113 & 68470 & 0.9 & $\begin{array}{r}9.964 \\
7 \\
\end{array}$ & 3.3 & 4.0824 & 3.4 & $\begin{array}{r}0.295 \\
0 \\
\end{array}$ & $\begin{array}{r}1 . \\
0\end{array}$ & $\begin{array}{r}0.2 \\
9 \\
\end{array}$ & $\begin{array}{r}1666 . \\
7 \\
\end{array}$ & 14.7 & 1650.8 & 28.1 & $\begin{array}{r}1630 . \\
6 \\
\end{array}$ & 61.2 & 1630.6 & 61.2 & $\begin{array}{r}102 . \\
2 \\
\end{array}$ \\
\hline NBH3-7 & 117 & 36595 & 2.3 & $\begin{array}{r}9.822 \\
0 \\
\end{array}$ & 3.2 & 3.6562 & 3.4 & $\begin{array}{r}0.260 \\
5 \\
\end{array}$ & $\begin{array}{r}1 . \\
3 \\
\end{array}$ & $\begin{array}{r}0.3 \\
8 \\
\end{array}$ & $\begin{array}{r}1492 . \\
2 \\
\end{array}$ & 17.3 & 1561.9 & 27.3 & $\begin{array}{r}1657 . \\
4 \\
\end{array}$ & 58.7 & 1657.4 & 58.7 & 90.0 \\
\hline NBH3-19 & 311 & 38140 & 1.1 & $\begin{array}{r}9.696 \\
4 \\
\end{array}$ & 3.3 & 3.9996 & 3.4 & $\begin{array}{r}0.281 \\
3 \\
\end{array}$ & $\begin{array}{r}1 . \\
0 \\
\end{array}$ & $\begin{array}{r}0.2 \\
9 \\
\end{array}$ & $\begin{array}{r}1597 . \\
8 \\
\end{array}$ & 14.2 & 1634.1 & 27.8 & $\begin{array}{r}1681 . \\
2 \\
\end{array}$ & 60.4 & 1681.2 & 60.4 & 95.0 \\
\hline NBH3-47 & 279 & 92480 & 2.0 & $\begin{array}{r}9.690 \\
3\end{array}$ & 1.1 & 4.0921 & 1.7 & $\begin{array}{r}0.287 \\
6\end{array}$ & $\begin{array}{r}1 . \\
3\end{array}$ & $\begin{array}{r}0.7 \\
7\end{array}$ & $\begin{array}{r}1629 . \\
5\end{array}$ & 19.0 & 1652.7 & 14.1 & $\begin{array}{r}1682 . \\
4\end{array}$ & 20.5 & 1682.4 & 20.5 & 96.9 \\
\hline NBH3-83 & $\begin{array}{r}206 \\
5 \\
\end{array}$ & $\begin{array}{r}37615 \\
5 \\
\end{array}$ & 1.9 & $\begin{array}{r}9.624 \\
2 \\
\end{array}$ & 1.3 & 4.0304 & 2.3 & $\begin{array}{r}0.281 \\
3 \\
\end{array}$ & $\begin{array}{r}1 . \\
9 \\
\end{array}$ & $\begin{array}{r}0.8 \\
3 \\
\end{array}$ & $\begin{array}{r}1598 . \\
0 \\
\end{array}$ & 27.3 & 1640.4 & 19.0 & $\begin{array}{r}1695 . \\
0 \\
\end{array}$ & 24.1 & 1695.0 & 24.1 & 94.3 \\
\hline NBH3-50 & 127 & 25634 & 2.0 & 9.619 & 2.3 & 4.1868 & 2.8 & 0.292 & 1. & 0.5 & 1652. & 24.2 & 1671.4 & 23.3 & 1695. & 42.4 & 1695.8 & 42.4 & 97.4 \\
\hline
\end{tabular}




\begin{tabular}{|c|c|c|c|c|c|c|c|c|c|c|c|c|c|c|c|c|c|c|c|}
\hline & 8 & 0 & & 9 & & & & 1 & 7 & 9 & 1 & & & & 8 & & & & \\
\hline NBH3-67 & 145 & 36395 & 1.4 & $\begin{array}{r}9.618 \\
6 \\
\end{array}$ & 4.7 & 4.1929 & 5.6 & $\begin{array}{r}0.292 \\
5 \\
\end{array}$ & $\begin{array}{c}3 . \\
1\end{array}$ & $\begin{array}{r}0.5 \\
5 \\
\end{array}$ & $\begin{array}{r}1654 . \\
0 \\
\end{array}$ & 45.2 & 1672.6 & 46.3 & $\begin{array}{r}1696 . \\
1 \\
\end{array}$ & 86.9 & 1696.1 & 86.9 & 97.5 \\
\hline NBH3-42 & 801 & $\begin{array}{r}15106 \\
5 \\
\end{array}$ & 1.9 & $\begin{array}{r}9.611 \\
0 \\
\end{array}$ & 1.2 & 4.1390 & 2.7 & $\begin{array}{r}0.288 \\
5 \\
\end{array}$ & $\begin{array}{r}2 . \\
4 \\
\end{array}$ & $\begin{array}{r}0.8 \\
9 \\
\end{array}$ & $\begin{array}{r}1634 . \\
1 \\
\end{array}$ & 35.2 & 1662.0 & 22.4 & $\begin{array}{r}1697 . \\
5 \\
\end{array}$ & 22.7 & 1697.5 & 22.7 & 96.3 \\
\hline $\mathrm{NBH} 3-49$ & 289 & 69370 & 1.6 & $\begin{array}{r}9.607 \\
3 \\
\end{array}$ & 1.2 & 4.1378 & 1.6 & $\begin{array}{r}0.288 \\
3 \\
\end{array}$ & $\begin{array}{r}1 . \\
0\end{array}$ & $\begin{array}{r}0.6 \\
3 \\
\end{array}$ & $\begin{array}{r}1633 . \\
1 \\
\end{array}$ & 14.4 & 1661.8 & 13.0 & $\begin{array}{r}1698 . \\
2 \\
\end{array}$ & 22.7 & 1698.2 & 22.7 & 96.2 \\
\hline NBH3-35 & $\begin{array}{r}278 \\
4 \\
\end{array}$ & $\begin{array}{r}34392 \\
5 \\
\end{array}$ & 0.9 & $\begin{array}{r}9.594 \\
9 \\
\end{array}$ & 1.6 & 3.9821 & 2.1 & $\begin{array}{r}0.277 \\
1 \\
\end{array}$ & $\begin{array}{r}1 . \\
4\end{array}$ & $\begin{array}{r}0.6 \\
7 \\
\end{array}$ & $\begin{array}{r}1576 . \\
8 \\
\end{array}$ & 19.9 & 1630.6 & 17.2 & $\begin{array}{r}1700 . \\
6 \\
\end{array}$ & 28.9 & 1700.6 & 28.9 & 92.7 \\
\hline $\mathrm{NBH} 3-22$ & 564 & 47400 & 1.9 & $\begin{array}{r}9.594 \\
7 \\
\end{array}$ & 2.1 & 4.0277 & 2.8 & $\begin{array}{r}0.280 \\
3 \\
\end{array}$ & $\begin{array}{r}1 . \\
8 \\
\end{array}$ & $\begin{array}{r}0.6 \\
5 \\
\end{array}$ & $\begin{array}{r}1592 . \\
8 \\
\end{array}$ & 25.3 & 1639.8 & 22.6 & $\begin{array}{r}1700 . \\
6 \\
\end{array}$ & 39.1 & 1700.6 & 39.1 & 93.7 \\
\hline $\mathrm{NBH} 3-10$ & 218 & 47830 & 0.9 & $\begin{array}{r}9.584 \\
3 \\
\end{array}$ & 3.3 & 4.0234 & 3.5 & $\begin{array}{r}0.279 \\
7 \\
\end{array}$ & $\begin{array}{l}1 . \\
0 \\
\end{array}$ & $\begin{array}{r}0.2 \\
9 \\
\end{array}$ & $\begin{array}{r}1589 . \\
7 \\
\end{array}$ & 14.1 & 1638.9 & 28.2 & $\begin{array}{r}1702 . \\
6 \\
\end{array}$ & 61.2 & 1702.6 & 61.2 & 93.4 \\
\hline NBH3-34 & 792 & $\begin{array}{r}14253 \\
0 \\
\end{array}$ & 1.9 & $\begin{array}{r}9.581 \\
5 \\
\end{array}$ & 1.0 & 4.0489 & 1.7 & $\begin{array}{r}0.281 \\
4 \\
\end{array}$ & $\begin{array}{r}1 . \\
4\end{array}$ & $\begin{array}{r}0.8 \\
2 \\
\end{array}$ & $\begin{array}{r}1598 . \\
2 \\
\end{array}$ & 20.2 & 1644.1 & 14.2 & $\begin{array}{r}1703 . \\
2 \\
\end{array}$ & 18.4 & 1703.2 & 18.4 & 93.8 \\
\hline $\mathrm{NBH} 3-38$ & 466 & $\begin{array}{r}10724 \\
5 \\
\end{array}$ & 1.5 & $\begin{array}{r}9.574 \\
7 \\
\end{array}$ & 1.2 & 4.0665 & 1.8 & $\begin{array}{r}0.282 \\
4 \\
\end{array}$ & $\begin{array}{r}1 . \\
4 \\
\end{array}$ & $\begin{array}{r}0.7 \\
5 \\
\end{array}$ & $\begin{array}{r}1603 . \\
4 \\
\end{array}$ & 19.3 & 1647.6 & 14.7 & $\begin{array}{r}1704 . \\
5 \\
\end{array}$ & 21.9 & 1704.5 & 21.9 & 94.1 \\
\hline NBH3-88 & 123 & 47625 & 1.1 & $\begin{array}{r}9.567 \\
5 \\
\end{array}$ & 2.0 & 4.4233 & 3.5 & $\begin{array}{r}0.306 \\
9 \\
\end{array}$ & $\begin{array}{r}2 . \\
9 \\
\end{array}$ & $\begin{array}{r}0.8 \\
2 \\
\end{array}$ & $\begin{array}{r}1725 . \\
6 \\
\end{array}$ & 43.1 & 1716.7 & 28.7 & $\begin{array}{r}1705 . \\
9 \\
\end{array}$ & 36.5 & 1705.9 & 36.5 & $\begin{array}{r}101 . \\
2 \\
\end{array}$ \\
\hline $\mathrm{NBH} 3-65$ & 164 & 64245 & 1.9 & $\begin{array}{r}9.566 \\
0 \\
\end{array}$ & 4.0 & 4.4502 & 4.3 & $\begin{array}{r}0.308 \\
7 \\
\end{array}$ & $\begin{array}{l}1 . \\
7 \\
\end{array}$ & $\begin{array}{r}0.4 \\
0 \\
\end{array}$ & $\begin{array}{r}1734 . \\
5 \\
\end{array}$ & 26.3 & 1721.7 & 35.8 & $\begin{array}{r}1706 . \\
2 \\
\end{array}$ & 72.7 & 1706.2 & 72.7 & $\begin{array}{r}101 . \\
7 \\
\end{array}$ \\
\hline $\mathrm{NBH} 3-14$ & $\begin{array}{r}112 \\
7 \\
\end{array}$ & $\begin{array}{r}13485 \\
0 \\
\end{array}$ & 1.5 & $\begin{array}{r}9.562 \\
8 \\
\end{array}$ & 2.3 & 3.8829 & 2.5 & $\begin{array}{r}0.269 \\
3 \\
\end{array}$ & $\begin{array}{l}. \\
0\end{array}$ & $\begin{array}{r}0.4 \\
1 \\
\end{array}$ & $\begin{array}{r}1537 . \\
3 \\
\end{array}$ & 13.7 & 1610.1 & 19.9 & $\begin{array}{r}1706 . \\
8 \\
\end{array}$ & 41.4 & 1706.8 & 41.4 & 90.1 \\
\hline NBH3-97 & $\begin{array}{r}139 \\
4 \\
\end{array}$ & $\begin{array}{r}0443 \\
0 \\
\end{array}$ & 1.5 & $\begin{array}{r}9.554 \\
9 \\
\end{array}$ & 2.3 & 4.4164 & 2.7 & $\begin{array}{r}0.306 \\
0 \\
\end{array}$ & $\begin{array}{r}1 . \\
4\end{array}$ & $\begin{array}{r}0.5 \\
3 \\
\end{array}$ & $\begin{array}{r}1721 . \\
2 \\
\end{array}$ & 21.8 & 1715.4 & 22.7 & $\begin{array}{r}1708 . \\
3 \\
\end{array}$ & 42.9 & 1708.3 & 42.9 & $\begin{array}{r}100 . \\
8 \\
\end{array}$ \\
\hline $\mathrm{NBH} 3-62$ & 150 & $\begin{array}{r}10720 \\
0 \\
\end{array}$ & 2.0 & $\begin{array}{r}9.510 \\
4 \\
\end{array}$ & 3.8 & 4.4140 & 4.1 & $\begin{array}{r}0.304 \\
5 \\
\end{array}$ & $\begin{array}{l}1 . \\
5 \\
\end{array}$ & $\begin{array}{r}0.3 \\
8 \\
\end{array}$ & $\begin{array}{r}1713 . \\
4 \\
\end{array}$ & 23.0 & 1715.0 & 33.6 & $\begin{array}{r}1716 . \\
9 \\
\end{array}$ & 69.1 & 1716.9 & 69.1 & 99.8 \\
\hline NBH3-16 & 240 & 83315 & 1.9 & $\begin{array}{r}9.505 \\
2 \\
\end{array}$ & 2.6 & 4.2441 & 2.9 & $\begin{array}{r}0.292 \\
6 \\
\end{array}$ & $\begin{array}{r}1 . \\
2 \\
\end{array}$ & $\begin{array}{r}0.4 \\
1 \\
\end{array}$ & $\begin{array}{r}1654 . \\
4 \\
\end{array}$ & 17.4 & 1682.6 & 23.8 & $\begin{array}{r}1717 . \\
9 \\
\end{array}$ & 48.5 & 1717.9 & 48.5 & 96.3 \\
\hline NBH3-9 & 815 & $\begin{array}{r}23313 \\
0 \\
\end{array}$ & 1.5 & $\begin{array}{r}9.489 \\
5 \\
\end{array}$ & 3.6 & 4.1271 & 4.1 & $\begin{array}{r}0.284 \\
0 \\
\end{array}$ & $\begin{array}{r}2 . \\
1 \\
\end{array}$ & $\begin{array}{r}0.5 \\
1 \\
\end{array}$ & $\begin{array}{r}1611 . \\
7\end{array}$ & 29.8 & 1659.7 & 33.7 & $\begin{array}{r}1720 . \\
9\end{array}$ & 65.2 & 1720.9 & 65.2 & 93.7 \\
\hline NBH3-87 & 589 & $\begin{array}{r}16070 \\
5 \\
\end{array}$ & 1.8 & $\begin{array}{r}9.473 \\
4 \\
\end{array}$ & 1.4 & 4.3896 & 1.9 & $\begin{array}{r}0.301 \\
6 \\
\end{array}$ & $\begin{array}{r}1 . \\
4\end{array}$ & $\begin{array}{r}0.7 \\
1 \\
\end{array}$ & $\begin{array}{r}1699 . \\
2 \\
\end{array}$ & 20.5 & 1710.4 & 16.0 & $\begin{array}{r}1724 . \\
0 \\
\end{array}$ & 25.0 & 1724.0 & 25.0 & 98.6 \\
\hline NBH3-11 & 405 & $\begin{array}{r}13492 \\
5 \\
\end{array}$ & 1.6 & $\begin{array}{r}9.465 \\
8 \\
\end{array}$ & 2.1 & 4.4787 & 2.3 & $\begin{array}{r}0.307 \\
5 \\
\end{array}$ & $\begin{array}{r}1 . \\
0\end{array}$ & $\begin{array}{r}0.4 \\
4 \\
\end{array}$ & $\begin{array}{r}1728 . \\
3 \\
\end{array}$ & 15.2 & 1727.0 & 19.0 & $\begin{array}{r}1725 . \\
5 \\
\end{array}$ & 37.8 & 1725.5 & 37.8 & $\begin{array}{r}100 . \\
2\end{array}$ \\
\hline NBH3-78 & 279 & $\begin{array}{r}13652 \\
0 \\
\end{array}$ & 1.7 & $\begin{array}{r}9.465 \\
7 \\
\end{array}$ & 1.6 & 4.5252 & 2.5 & $\begin{array}{r}0.310 \\
7 \\
\end{array}$ & $\begin{array}{r}1 . \\
9 \\
\end{array}$ & $\begin{array}{r}0.7 \\
8 \\
\end{array}$ & $\begin{array}{r}1744 . \\
0 \\
\end{array}$ & 29.3 & 1735.6 & 20.5 & $\begin{array}{r}1725 . \\
5 \\
\end{array}$ & 28.5 & 1725.5 & 28.5 & $\begin{array}{r}101 . \\
1 \\
\end{array}$ \\
\hline NBH3-3 & 226 & 97555 & 2.3 & $\begin{array}{r}9.463 \\
5 \\
\end{array}$ & 2.7 & 4.5684 & 3.2 & $\begin{array}{r}0.313 \\
6 \\
\end{array}$ & $\begin{array}{r}. \\
8 \\
\end{array}$ & $\begin{array}{r}0.5 \\
5 \\
\end{array}$ & $\begin{array}{r}1758 . \\
2 \\
\end{array}$ & 27.2 & 1743.5 & 26.8 & $\begin{array}{r}1726 . \\
0 \\
\end{array}$ & 49.2 & 1726.0 & 49.2 & $\begin{array}{r}101 . \\
9 \\
\end{array}$ \\
\hline NBH3-81 & 319 & $\begin{array}{r}12252 \\
0 \\
\end{array}$ & 1.1 & $\begin{array}{r}9.432 \\
7 \\
\end{array}$ & 1.5 & 4.4898 & 2.0 & $\begin{array}{r}0.307 \\
2 \\
\end{array}$ & $\begin{array}{c}1 . \\
3\end{array}$ & $\begin{array}{r}0.6 \\
6 \\
\end{array}$ & $\begin{array}{r}1726 . \\
7 \\
\end{array}$ & 19.8 & 1729.1 & 16.5 & $\begin{array}{r}1732 . \\
0 \\
\end{array}$ & 27.3 & 1732.0 & 27.3 & 99.7 \\
\hline $\mathrm{NBH} 3-25$ & 210 & $\begin{array}{r}10232 \\
5 \\
\end{array}$ & 1.6 & $\begin{array}{r}9.417 \\
3 \\
\end{array}$ & 2.0 & 4.2468 & 2.3 & $\begin{array}{r}0.290 \\
1 \\
\end{array}$ & $\begin{array}{r}1 . \\
2 \\
\end{array}$ & $\begin{array}{r}0.5 \\
2 \\
\end{array}$ & $\begin{array}{r}1641 . \\
8 \\
\end{array}$ & 17.2 & 1683.1 & 19.0 & $\begin{array}{r}1735 . \\
0 \\
\end{array}$ & 36.3 & 1735.0 & 36.3 & 94.6 \\
\hline NBH3-61 & 347 & $\begin{array}{r}35143 \\
5\end{array}$ & 1.1 & $\begin{array}{r}9.392 \\
3\end{array}$ & 3.6 & 4.6653 & 3.9 & $\begin{array}{r}0.317 \\
8\end{array}$ & $\begin{array}{r}1 . \\
5\end{array}$ & $\begin{array}{r}0.3 \\
9\end{array}$ & $\begin{array}{r}1779 . \\
0\end{array}$ & 23.3 & 1761.0 & 32.5 & $\begin{array}{r}1739 . \\
8\end{array}$ & 65.6 & 1739.8 & 65.6 & $\begin{array}{r}102 . \\
2\end{array}$ \\
\hline NBH3-99 & $\begin{array}{r}212 \\
2 \\
\end{array}$ & $\begin{array}{r}15806 \\
0 \\
\end{array}$ & 1.9 & $\begin{array}{r}9.389 \\
1 \\
\end{array}$ & 2.4 & 3.7334 & 5.1 & $\begin{array}{r}0.254 \\
2 \\
\end{array}$ & $\begin{array}{r}4 . \\
5 \\
\end{array}$ & $\begin{array}{r}0.8 \\
8 \\
\end{array}$ & $\begin{array}{r}1460 . \\
3 \\
\end{array}$ & 58.3 & 1578.6 & 40.7 & $\begin{array}{r}1740 . \\
4 \\
\end{array}$ & 44.7 & 1740.4 & 44.7 & 83.9 \\
\hline $\mathrm{NBH} 3-12$ & 830 & $\begin{array}{r}15088 \\
5 \\
\end{array}$ & 7.4 & $\begin{array}{r}9.383 \\
4 \\
\end{array}$ & 3.5 & 3.5834 & 4.8 & $\begin{array}{r}0.243 \\
9 \\
\end{array}$ & $\begin{array}{l}3 . \\
3 \\
\end{array}$ & $\begin{array}{r}0.6 \\
9 \\
\end{array}$ & $\begin{array}{r}1406 . \\
8 \\
\end{array}$ & 41.2 & 1545.9 & 37.8 & $\begin{array}{r}1741 . \\
6 \\
\end{array}$ & 63.4 & 1741.6 & 63.4 & 80.8 \\
\hline
\end{tabular}




\begin{tabular}{|c|c|c|c|c|c|c|c|c|c|c|c|c|c|c|c|c|c|c|c|}
\hline NBH3-69 & 224 & $\begin{array}{r}12314 \\
5 \\
\end{array}$ & 1.6 & $\begin{array}{r}9.345 \\
0 \\
\end{array}$ & 3.3 & 4.4250 & 3.5 & $\begin{array}{r}0.299 \\
9 \\
\end{array}$ & $\begin{array}{r}1 . \\
2 \\
\end{array}$ & $\begin{array}{r}0.3 \\
4 \\
\end{array}$ & $\begin{array}{r}1690 . \\
9 \\
\end{array}$ & 17.8 & 1717.0 & 29.3 & $\begin{array}{r}1749 . \\
1 \\
\end{array}$ & 61.0 & 1749.1 & 61.0 & 96.7 \\
\hline NBH3-58 & $\begin{array}{r}281 \\
8\end{array}$ & $\begin{array}{r}45993 \\
5\end{array}$ & 1.8 & $\begin{array}{r}9.332 \\
5\end{array}$ & 1.0 & 4.4510 & 2.5 & $\begin{array}{r}0.301 \\
3\end{array}$ & $\begin{array}{r}2 . \\
3\end{array}$ & $\begin{array}{r}0.9 \\
2\end{array}$ & $\begin{array}{r}1697 . \\
6\end{array}$ & 34.8 & 1721.9 & 21.0 & $\begin{array}{r}1751 . \\
5\end{array}$ & 18.3 & 1751.5 & 18.3 & 96.9 \\
\hline NBH3-85 & 294 & 94875 & 2.2 & $\begin{array}{r}9.313 \\
2 \\
\end{array}$ & 1.1 & 4.5333 & 1.5 & $\begin{array}{r}0.306 \\
2 \\
\end{array}$ & $\begin{array}{r}1 . \\
0\end{array}$ & $\begin{array}{r}0.6 \\
6 \\
\end{array}$ & $\begin{array}{r}1722 . \\
0 \\
\end{array}$ & 15.1 & 1737.1 & 12.6 & $\begin{array}{r}1755 . \\
3 \\
\end{array}$ & 20.7 & 1755.3 & 20.7 & 98.1 \\
\hline NBH3-8 & 167 & 78975 & 0.9 & $\begin{array}{r}9.312 \\
8 \\
\end{array}$ & 5.2 & 4.5960 & 5.6 & $\begin{array}{r}0.310 \\
4 \\
\end{array}$ & $\begin{array}{r}2 . \\
0 \\
\end{array}$ & $\begin{array}{r}0.3 \\
5 \\
\end{array}$ & $\begin{array}{r}1742 . \\
8 \\
\end{array}$ & 29.9 & 1748.5 & 46.5 & $\begin{array}{r}1755 . \\
4 \\
\end{array}$ & 95.4 & 1755.4 & 95.4 & 99.3 \\
\hline NBH3-94 & 405 & $\begin{array}{r}15927 \\
5 \\
\end{array}$ & 1.6 & $\begin{array}{r}9.219 \\
5 \\
\end{array}$ & 4.5 & 4.5424 & 4.7 & $\begin{array}{r}0.303 \\
7 \\
\end{array}$ & $\begin{array}{r}1 . \\
4 \\
\end{array}$ & $\begin{array}{r}0.3 \\
0 \\
\end{array}$ & $\begin{array}{r}1709 . \\
8 \\
\end{array}$ & 20.7 & 1738.8 & 38.9 & $\begin{array}{r}1773 . \\
8 \\
\end{array}$ & 81.4 & 1773.8 & 81.4 & 96.4 \\
\hline NBH3-84 & 298 & 88030 & 1.9 & $\begin{array}{r}9.183 \\
2 \\
\end{array}$ & 1.0 & 4.8280 & 1.4 & $\begin{array}{r}0.321 \\
6\end{array}$ & $\begin{array}{r}1 . \\
0\end{array}$ & $\begin{array}{r}0.7 \\
1 \\
\end{array}$ & $\begin{array}{r}1797 . \\
3 \\
\end{array}$ & 15.7 & 1789.8 & 11.9 & $\begin{array}{r}1781 . \\
0\end{array}$ & 18.2 & 1781.0 & 18.2 & $\begin{array}{r}100 . \\
9\end{array}$ \\
\hline NBH3-68 & 577 & $\begin{array}{r}38565 \\
5 \\
\end{array}$ & 1.1 & $\begin{array}{r}9.147 \\
7 \\
\end{array}$ & 2.2 & 4.8534 & 2.9 & $\begin{array}{r}0.322 \\
0 \\
\end{array}$ & $\begin{array}{r}1 . \\
8\end{array}$ & $\begin{array}{r}0.6 \\
4 \\
\end{array}$ & $\begin{array}{r}1799 . \\
5 \\
\end{array}$ & 28.7 & 1794.2 & 24.1 & $\begin{array}{r}1788 . \\
0 \\
\end{array}$ & 40.1 & 1788.0 & 40.1 & $\begin{array}{r}100 . \\
6 \\
\end{array}$ \\
\hline NBH3-77 & 132 & 31535 & 1.5 & $\begin{array}{r}8.996 \\
1 \\
\end{array}$ & 4.0 & 4.9072 & 4.3 & $\begin{array}{r}0.320 \\
2 \\
\end{array}$ & $\begin{array}{l}1 . \\
5 \\
\end{array}$ & $\begin{array}{r}0.3 \\
4 \\
\end{array}$ & $\begin{array}{r}1790 . \\
6 \\
\end{array}$ & 23.1 & 1803.5 & 36.2 & $\begin{array}{r}1818 . \\
4 \\
\end{array}$ & 73.2 & 1818.4 & 73.2 & 98.5 \\
\hline $\mathrm{NBH} 3-74$ & 123 & 49070 & 0.8 & $\begin{array}{r}8.979 \\
8 \\
\end{array}$ & 1.3 & 4.9215 & 1.8 & $\begin{array}{r}0.320 \\
5 \\
\end{array}$ & $\begin{array}{r}1 . \\
2 \\
\end{array}$ & $\begin{array}{r}0.6 \\
8 \\
\end{array}$ & $\begin{array}{r}1792 . \\
3 \\
\end{array}$ & 19.2 & 1805.9 & 15.2 & $\begin{array}{r}1821 . \\
7\end{array}$ & 24.0 & 1821.7 & 24.0 & 98.4 \\
\hline $\mathrm{NBH} 3-71$ & 447 & $\begin{array}{r}11701 \\
0 \\
\end{array}$ & 1.3 & $\begin{array}{r}8.934 \\
4 \\
\end{array}$ & 2.5 & 4.7252 & 3.2 & $\begin{array}{r}0.306 \\
2 \\
\end{array}$ & $\begin{array}{r}2 . \\
0 \\
\end{array}$ & $\begin{array}{r}0.6 \\
3 \\
\end{array}$ & $\begin{array}{r}1721 . \\
9 \\
\end{array}$ & 30.7 & 1771.7 & 27.1 & $\begin{array}{r}1830 . \\
9 \\
\end{array}$ & 45.5 & 1830.9 & 45.5 & 94.0 \\
\hline NBH3-2 & 344 & $\begin{array}{r}14403 \\
0 \\
\end{array}$ & 8.1 & $\begin{array}{r}8.932 \\
8 \\
\end{array}$ & 4.3 & 5.2109 & 4.4 & $\begin{array}{r}0.337 \\
6 \\
\end{array}$ & $\begin{array}{r}1 . \\
1 \\
\end{array}$ & $\begin{array}{r}0.2 \\
4 \\
\end{array}$ & $\begin{array}{r}1875 . \\
1 \\
\end{array}$ & 17.2 & 1854.4 & 37.4 & $\begin{array}{r}1831 . \\
3 \\
\end{array}$ & 77.2 & 1831.3 & 77.2 & $\begin{array}{r}102 . \\
4 \\
\end{array}$ \\
\hline NBH3-59 & 513 & 68145 & 1.9 & $\begin{array}{r}8.875 \\
9 \\
\end{array}$ & 1.9 & 4.5500 & 2.7 & $\begin{array}{r}0.292 \\
9 \\
\end{array}$ & $\begin{array}{c}1 . \\
9 \\
\end{array}$ & $\begin{array}{r}0.7 \\
0 \\
\end{array}$ & $\begin{array}{r}1656 . \\
0 \\
\end{array}$ & 27.5 & 1740.2 & 22.3 & $\begin{array}{r}1842 . \\
8 \\
\end{array}$ & 34.6 & 1842.8 & 34.6 & 89.9 \\
\hline $\mathrm{NBH} 3-24$ & 526 & 10940 & 1.2 & $\begin{array}{r}8.872 \\
0 \\
\end{array}$ & 4.9 & 3.7669 & 5.2 & $\begin{array}{r}0.242 \\
4 \\
\end{array}$ & $\begin{array}{r}1 . \\
9 \\
\end{array}$ & $\begin{array}{r}0.3 \\
6 \\
\end{array}$ & $\begin{array}{r}1399 . \\
1 \\
\end{array}$ & 23.4 & 1585.7 & 41.8 & $\begin{array}{r}1843 . \\
6 \\
\end{array}$ & 88.0 & 1843.6 & 88.0 & 75.9 \\
\hline NBH3-53 & 258 & 87090 & 2.3 & $\begin{array}{r}8.859 \\
7 \\
\end{array}$ & 1.5 & 4.7936 & 2.1 & $\begin{array}{r}0.308 \\
0 \\
\end{array}$ & $\begin{array}{r}1 . \\
4 \\
\end{array}$ & $\begin{array}{r}0.6 \\
8 \\
\end{array}$ & $\begin{array}{r}1731 . \\
0 \\
\end{array}$ & 21.3 & 1783.8 & 17.4 & $\begin{array}{r}1846 . \\
1 \\
\end{array}$ & 27.5 & 1846.1 & 27.5 & 93.8 \\
\hline NBH3-86 & 111 & 49335 & 0.8 & $\begin{array}{r}8.820 \\
8 \\
\end{array}$ & 2.2 & 5.1702 & 2.8 & $\begin{array}{r}0.330 \\
8 \\
\end{array}$ & $\begin{array}{l}1 . \\
7\end{array}$ & $\begin{array}{r}0.6 \\
2 \\
\end{array}$ & $\begin{array}{r}1842 . \\
1 \\
\end{array}$ & 27.6 & 1847.7 & 23.6 & $\begin{array}{r}1854 . \\
1 \\
\end{array}$ & 39.4 & 1854.1 & 39.4 & 99.4 \\
\hline NBH3-15 & 791 & $\begin{array}{r}13234 \\
5 \\
\end{array}$ & 3.3 & $\begin{array}{r}8.729 \\
3 \\
\end{array}$ & 2.2 & 4.7711 & 2.4 & $\begin{array}{r}0.302 \\
1 \\
\end{array}$ & $\begin{array}{r}1 . \\
0\end{array}$ & $\begin{array}{r}0.4 \\
2 \\
\end{array}$ & $\begin{array}{r}1701 . \\
5 \\
\end{array}$ & 15.0 & 1779.8 & 20.1 & $\begin{array}{r}1872 . \\
9 \\
\end{array}$ & 39.3 & 1872.9 & 39.3 & 90.8 \\
\hline NBH3-96 & 199 & 71330 & 1.8 & $\begin{array}{r}8.241 \\
1 \\
\end{array}$ & 2.8 & 5.7241 & 3.2 & $\begin{array}{r}0.342 \\
1 \\
\end{array}$ & $\begin{array}{r}1 . \\
4 \\
\end{array}$ & $\begin{array}{r}0.4 \\
4 \\
\end{array}$ & $\begin{array}{r}1896 . \\
9 \\
\end{array}$ & 22.8 & 1935.0 & 27.3 & $\begin{array}{r}1976 . \\
0 \\
\end{array}$ & 50.6 & 1976.0 & 50.6 & 96.0 \\
\hline NBH3-30 & 503 & 71760 & 2.0 & $\begin{array}{r}8.238 \\
8 \\
\end{array}$ & 1.0 & 5.1661 & 1.7 & $\begin{array}{r}0.308 \\
7 \\
\end{array}$ & $\begin{array}{r}1 . \\
4 \\
\end{array}$ & $\begin{array}{r}0.8 \\
2 \\
\end{array}$ & $\begin{array}{r}1734 . \\
3 \\
\end{array}$ & 21.7 & 1847.1 & 14.8 & $\begin{array}{r}1976 . \\
6 \\
\end{array}$ & 17.8 & 1976.6 & 17.8 & 87.7 \\
\hline NBH3-82 & 68 & 28545 & 0.9 & $\begin{array}{r}7.199 \\
3 \\
\end{array}$ & 3.0 & 5.9493 & 3.1 & $\begin{array}{r}0.310 \\
6 \\
\end{array}$ & $\begin{array}{r}1 . \\
0\end{array}$ & $\begin{array}{r}0.3 \\
2 \\
\end{array}$ & $\begin{array}{r}1743 . \\
8 \\
\end{array}$ & 15.3 & 1968.5 & 27.2 & $\begin{array}{r}2213 . \\
6 \\
\end{array}$ & 51.4 & 2213.6 & 51.4 & 78.8 \\
\hline NBH3-92 & 444 & $\begin{array}{r}20682 \\
0 \\
\end{array}$ & 0.8 & $\begin{array}{r}6.355 \\
0 \\
\end{array}$ & 1.4 & 9.5263 & 1.7 & $\begin{array}{r}0.439 \\
1 \\
\end{array}$ & $\begin{array}{r}1 . \\
1\end{array}$ & $\begin{array}{r}0.6 \\
1 \\
\end{array}$ & $\begin{array}{r}2346 . \\
5 \\
\end{array}$ & 20.8 & 2390.1 & 16.0 & $\begin{array}{r}2427 . \\
4 \\
\end{array}$ & 23.4 & 2427.4 & 23.4 & 96.7 \\
\hline NBH3-70 & 280 & $\begin{array}{r}16294 \\
0 \\
\end{array}$ & 0.8 & $\begin{array}{r}6.302 \\
0 \\
\end{array}$ & 4.2 & 9.5192 & 4.8 & $\begin{array}{r}0.435 \\
1 \\
\end{array}$ & $\begin{array}{r}2 . \\
3 \\
\end{array}$ & $\begin{array}{r}0.4 \\
7 \\
\end{array}$ & $\begin{array}{r}2328 . \\
6 \\
\end{array}$ & 44.0 & 2389.4 & 44.0 & $\begin{array}{r}2441 . \\
6 \\
\end{array}$ & 71.5 & 2441.6 & 71.5 & 95.4 \\
\hline NBH3-40 & 161 & 55875 & 1.2 & $\begin{array}{r}6.149 \\
7 \\
\end{array}$ & 2.2 & $\begin{array}{r}10.226 \\
1 \\
\end{array}$ & 2.4 & $\begin{array}{r}0.456 \\
1 \\
\end{array}$ & $\begin{array}{r}1 . \\
0 \\
\end{array}$ & $\begin{array}{r}0.4 \\
2 \\
\end{array}$ & $\begin{array}{r}2422 . \\
3 \\
\end{array}$ & 20.2 & 2455.4 & 22.2 & $\begin{array}{r}2483 . \\
0 \\
\end{array}$ & 36.8 & 2483.0 & 36.8 & 97.6 \\
\hline NBH3-56 & 245 & $\begin{array}{r}15367 \\
0 \\
\end{array}$ & 0.8 & $\begin{array}{r}6.077 \\
0 \\
\end{array}$ & 2.0 & $\begin{array}{r}10.080 \\
4 \\
\end{array}$ & 2.3 & $\begin{array}{r}0.444 \\
3 \\
\end{array}$ & $\begin{array}{r}1 . \\
0 \\
\end{array}$ & $\begin{array}{r}0.4 \\
4 \\
\end{array}$ & $\begin{array}{r}2369 . \\
8 \\
\end{array}$ & 19.8 & 2442.2 & 21.0 & $\begin{array}{r}2503 . \\
0 \\
\end{array}$ & 34.3 & 2503.0 & 34.3 & 94.7 \\
\hline $\mathrm{NBH} 3-76$ & 56 & 48085 & 0.6 & $\begin{array}{r}5.990 \\
8 \\
\end{array}$ & 2.3 & $\begin{array}{r}11.220 \\
4 \\
\end{array}$ & 3.0 & $\begin{array}{r}0.487 \\
5 \\
\end{array}$ & $\begin{array}{r}1 . \\
9 \\
\end{array}$ & $\begin{array}{r}0.6 \\
3 \\
\end{array}$ & $\begin{array}{r}2560 . \\
0 \\
\end{array}$ & 40.1 & 2541.6 & 28.0 & $\begin{array}{r}2527 . \\
0 \\
\end{array}$ & 39.1 & 2527.0 & 39.1 & $\begin{array}{r}101 . \\
3 \\
\end{array}$ \\
\hline NBH3-20 & 452 & 50175 & 1.1 & 3.936 & 2.0 & 21.597 & 2.3 & 0.616 & 1. & 0.5 & 3096. & 29.3 & 3165.8 & 22.2 & 3209. & 30.8 & 3209.9 & 30.8 & 96.5 \\
\hline
\end{tabular}




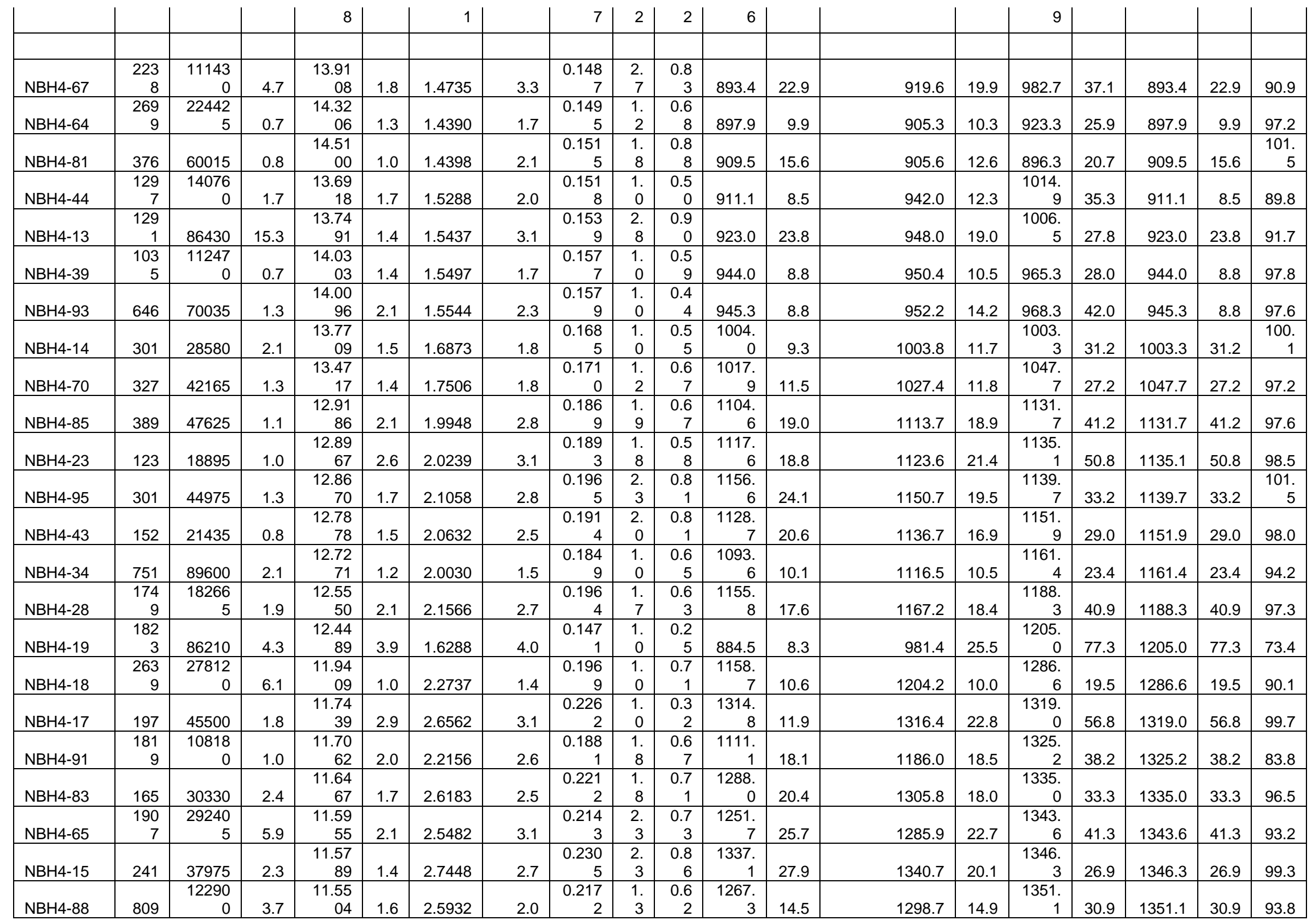




\begin{tabular}{|c|c|c|c|c|c|c|c|c|c|c|c|c|c|c|c|c|c|c|c|}
\hline $\mathrm{NBH} 4-82$ & 478 & 71040 & 2.4 & $\begin{array}{r}11.52 \\
97 \\
\end{array}$ & 1.1 & 2.6605 & 2.1 & $\begin{array}{r}0.222 \\
5 \\
\end{array}$ & $\begin{array}{r}1 . \\
8 \\
\end{array}$ & $\begin{array}{r}0.8 \\
6 \\
\end{array}$ & $\begin{array}{r}1294 . \\
9 \\
\end{array}$ & 20.9 & 1317.6 & 15.3 & $\begin{array}{r}1354 . \\
5 \\
\end{array}$ & 20.6 & 1354.5 & 20.6 & 95.6 \\
\hline NBH4-55 & 253 & 46415 & 1.1 & $\begin{array}{r}11.51 \\
37 \\
\end{array}$ & 2.9 & 2.7559 & 3.1 & $\begin{array}{r}0.230 \\
1 \\
\end{array}$ & $\begin{array}{l}1 . \\
0\end{array}$ & $\begin{array}{r}0.3 \\
2 \\
\end{array}$ & $\begin{array}{r}1335 . \\
2 \\
\end{array}$ & 12.1 & 1343.7 & 23.0 & $\begin{array}{r}1357 . \\
2 \\
\end{array}$ & 56.3 & 1357.2 & 56.3 & 98.4 \\
\hline $\mathrm{NBH} 4-92$ & $\begin{array}{r}107 \\
9 \\
\end{array}$ & $\begin{array}{r}10538 \\
5 \\
\end{array}$ & 2.1 & $\begin{array}{r}11.48 \\
36 \\
\end{array}$ & 1.8 & 2.4789 & 3.3 & $\begin{array}{r}0.206 \\
5 \\
\end{array}$ & $\begin{array}{r}2 . \\
8 \\
\end{array}$ & $\begin{array}{r}0.8 \\
4 \\
\end{array}$ & $\begin{array}{r}1209 . \\
9 \\
\end{array}$ & 30.3 & 1265.9 & 23.7 & $\begin{array}{r}1362 . \\
3 \\
\end{array}$ & 34.5 & 1362.3 & 34.5 & 88.8 \\
\hline NBH4-87 & 609 & 81565 & 1.4 & $\begin{array}{r}11.48 \\
18 \\
\end{array}$ & 1.0 & 2.5731 & 2.8 & $\begin{array}{r}0.214 \\
3 \\
\end{array}$ & $\begin{array}{r}2 . \\
7 \\
\end{array}$ & $\begin{array}{r}0.9 \\
4 \\
\end{array}$ & $\begin{array}{r}1251 . \\
6 \\
\end{array}$ & 30.3 & 1293.0 & 20.8 & $\begin{array}{r}1362 . \\
6 \\
\end{array}$ & 19.3 & 1362.6 & 19.3 & 91.9 \\
\hline $\mathrm{NBH} 4-46$ & 369 & 53415 & 2.2 & $\begin{array}{r}11.42 \\
43 \\
\end{array}$ & 2.4 & 2.7204 & 3.4 & $\begin{array}{r}0.225 \\
4 \\
\end{array}$ & $\begin{array}{r}2 . \\
5 \\
\end{array}$ & $\begin{array}{r}0.7 \\
3 \\
\end{array}$ & $\begin{array}{r}1310 . \\
4\end{array}$ & 29.5 & 1334.0 & 25.5 & $\begin{array}{r}1372 . \\
2 \\
\end{array}$ & 45.4 & 1372.2 & 45.4 & 95.5 \\
\hline NBH4-89 & $\begin{array}{r}103 \\
5 \\
\end{array}$ & $\begin{array}{r}17516 \\
0 \\
\end{array}$ & 4.2 & $\begin{array}{r}11.36 \\
39 \\
\end{array}$ & 1.0 & 2.5144 & 1.4 & $\begin{array}{r}0.207 \\
2 \\
\end{array}$ & $\begin{array}{l}1 . \\
0\end{array}$ & $\begin{array}{r}0.7 \\
1 \\
\end{array}$ & $\begin{array}{r}1214 . \\
1 \\
\end{array}$ & 11.1 & 1276.2 & 10.3 & $\begin{array}{r}1382 . \\
4 \\
\end{array}$ & 19.2 & 1382.4 & 19.2 & 87.8 \\
\hline NBH4-4 & 86 & 24345 & 2.1 & $\begin{array}{r}11.28 \\
86 \\
\end{array}$ & 2.8 & 2.9436 & 4.9 & $\begin{array}{r}0.241 \\
0 \\
\end{array}$ & $\begin{array}{r}4 . \\
0\end{array}$ & $\begin{array}{r}0.8 \\
2 \\
\end{array}$ & $\begin{array}{r}1391 . \\
9 \\
\end{array}$ & 50.5 & 1393.2 & 37.1 & $\begin{array}{r}1395 . \\
2 \\
\end{array}$ & 53.3 & 1395.2 & 53.3 & 99.8 \\
\hline $\mathrm{NBH} 4-74$ & 391 & 75950 & 3.6 & $\begin{array}{r}11.17 \\
28 \\
\end{array}$ & 1.2 & 3.0320 & 1.8 & $\begin{array}{r}0.245 \\
7 \\
\end{array}$ & $\begin{array}{r}1 . \\
3\end{array}$ & $\begin{array}{r}0.7 \\
6 \\
\end{array}$ & $\begin{array}{r}1416 . \\
2 \\
\end{array}$ & 17.0 & 1415.7 & 13.5 & $\begin{array}{r}1414 . \\
9 \\
\end{array}$ & 22.2 & 1414.9 & 22.2 & $\begin{array}{r}100 . \\
1 \\
\end{array}$ \\
\hline NBH4-57 & $\begin{array}{r}275 \\
6 \\
\end{array}$ & $\begin{array}{r}33144 \\
5 \\
\end{array}$ & 2.2 & $\begin{array}{r}11.02 \\
90 \\
\end{array}$ & 2.1 & 2.8216 & 2.3 & $\begin{array}{r}0.225 \\
7 \\
\end{array}$ & $\begin{array}{l}1 . \\
0\end{array}$ & $\begin{array}{r}0.4 \\
3 \\
\end{array}$ & $\begin{array}{r}1311 . \\
9 \\
\end{array}$ & 11.9 & 1361.3 & 17.4 & $\begin{array}{r}1439 . \\
7 \\
\end{array}$ & 39.8 & 1439.7 & 39.8 & 91.1 \\
\hline $\mathrm{NBH} 4-36$ & 323 & 40825 & 5.0 & $\begin{array}{r}10.98 \\
85 \\
\end{array}$ & 3.5 & 2.8237 & 5.2 & $\begin{array}{r}0.225 \\
0 \\
\end{array}$ & $\begin{array}{r}3 . \\
8 \\
\end{array}$ & $\begin{array}{r}0.7 \\
3 \\
\end{array}$ & $\begin{array}{r}1308 . \\
5 \\
\end{array}$ & 44.6 & 1361.9 & 38.7 & $\begin{array}{r}1446 . \\
7 \\
\end{array}$ & 67.1 & 1446.7 & 67.1 & 90.4 \\
\hline NBH4-59 & 528 & $\begin{array}{r}12101 \\
5 \\
\end{array}$ & 2.0 & $\begin{array}{r}10.95 \\
91 \\
\end{array}$ & 2.3 & 3.1404 & 3.1 & $\begin{array}{r}0.249 \\
6 \\
\end{array}$ & $\begin{array}{r}2 . \\
1 \\
\end{array}$ & $\begin{array}{r}0.6 \\
8 \\
\end{array}$ & $\begin{array}{r}1436 . \\
4 \\
\end{array}$ & 27.0 & 1442.6 & 23.7 & $\begin{array}{r}1451 . \\
8 \\
\end{array}$ & 42.8 & 1451.8 & 42.8 & 98.9 \\
\hline $\mathrm{NBH} 4-66$ & 165 & 7205 & 1.2 & $\begin{array}{r}10.93 \\
49 \\
\end{array}$ & 6.0 & 2.4174 & 6.2 & $\begin{array}{r}0.191 \\
7 \\
\end{array}$ & $\begin{array}{l}1 . \\
8\end{array}$ & $\begin{array}{r}0.2 \\
9 \\
\end{array}$ & $\begin{array}{r}1130 . \\
7\end{array}$ & 18.7 & 1247.8 & 44.9 & $\begin{array}{r}1456 . \\
0 \\
\end{array}$ & $\begin{array}{r}113 . \\
9 \\
\end{array}$ & 1456.0 & $\begin{array}{r}113 . \\
9 \\
\end{array}$ & 77.7 \\
\hline $\mathrm{NBH} 4-35$ & 158 & 29855 & 1.7 & $\begin{array}{r}10.80 \\
42 \\
\end{array}$ & 1.2 & 3.2975 & 1.6 & $\begin{array}{r}0.258 \\
4 \\
\end{array}$ & $\begin{array}{l}1 . \\
0\end{array}$ & $\begin{array}{r}0.6 \\
4 \\
\end{array}$ & $\begin{array}{r}1481 . \\
6 \\
\end{array}$ & 13.2 & 1480.5 & 12.2 & $\begin{array}{r}1478 . \\
8 \\
\end{array}$ & 23.0 & 1478.8 & 23.0 & $\begin{array}{r}100 . \\
2 \\
\end{array}$ \\
\hline $\mathrm{NBH} 4-47$ & 209 & 40635 & 2.4 & $\begin{array}{r}10.69 \\
14 \\
\end{array}$ & 1.3 & 3.0644 & 1.7 & $\begin{array}{r}0.237 \\
6 \\
\end{array}$ & $\begin{array}{l}1 . \\
0\end{array}$ & $\begin{array}{r}0.6 \\
0 \\
\end{array}$ & $\begin{array}{r}1374 . \\
3 \\
\end{array}$ & 12.4 & 1423.8 & 12.7 & $\begin{array}{r}1498 . \\
7 \\
\end{array}$ & 25.2 & 1498.7 & 25.2 & 91.7 \\
\hline NBH4-29 & 97 & 20985 & 1.1 & $\begin{array}{r}10.51 \\
40 \\
\end{array}$ & 2.6 & 3.5791 & 3.1 & $\begin{array}{r}0.272 \\
9 \\
\end{array}$ & $\begin{array}{l}1 . \\
7\end{array}$ & $\begin{array}{r}0.5 \\
4 \\
\end{array}$ & $\begin{array}{r}1555 . \\
6 \\
\end{array}$ & 23.2 & 1544.9 & 24.8 & $\begin{array}{r}1530 . \\
3 \\
\end{array}$ & 49.7 & 1530.3 & 49.7 & $\begin{array}{r}101 . \\
7 \\
\end{array}$ \\
\hline NBH4-5 & 254 & 46440 & 1.6 & $\begin{array}{r}10.42 \\
23 \\
\end{array}$ & 3.1 & 3.6549 & 3.2 & $\begin{array}{r}0.276 \\
3 \\
\end{array}$ & $\begin{array}{l}1 . \\
0\end{array}$ & $\begin{array}{r}0.3 \\
1 \\
\end{array}$ & $\begin{array}{r}1572 . \\
6 \\
\end{array}$ & 14.0 & 1561.6 & 25.6 & $\begin{array}{r}1546 . \\
7 \\
\end{array}$ & 57.3 & 1546.7 & 57.3 & $\begin{array}{r}101 . \\
7 \\
\end{array}$ \\
\hline $\mathrm{NBH} 4-73$ & $\begin{array}{r}259 \\
2 \\
\end{array}$ & $\begin{array}{r}22958 \\
5 \\
\end{array}$ & 1.2 & $\begin{array}{r}10.36 \\
14 \\
\end{array}$ & 1.8 & 2.7932 & 3.8 & $\begin{array}{r}0.209 \\
9 \\
\end{array}$ & $\begin{array}{r}3 . \\
3 \\
\end{array}$ & $\begin{array}{r}0.8 \\
7 \\
\end{array}$ & $\begin{array}{r}1228 . \\
3 \\
\end{array}$ & 36.7 & 1353.7 & 28.1 & $\begin{array}{r}1557 . \\
7 \\
\end{array}$ & 34.2 & 1557.7 & 34.2 & 78.9 \\
\hline NBH4-68 & 381 & 56325 & 1.4 & $\begin{array}{r}10.34 \\
77 \\
\end{array}$ & 2.1 & 3.2917 & 2.4 & $\begin{array}{r}0.247 \\
0 \\
\end{array}$ & $\begin{array}{r}1 . \\
2 \\
\end{array}$ & $\begin{array}{r}0.4 \\
9 \\
\end{array}$ & $\begin{array}{r}1423 . \\
2 \\
\end{array}$ & 14.9 & 1479.1 & 18.7 & $\begin{array}{r}1560 . \\
2 \\
\end{array}$ & 39.2 & 1560.2 & 39.2 & 91.2 \\
\hline NBH4-69 & 272 & 61520 & 1.6 & $\begin{array}{r}10.24 \\
21 \\
\end{array}$ & 1.0 & 3.5783 & 1.6 & $\begin{array}{r}0.265 \\
8 \\
\end{array}$ & $\begin{array}{r}1 . \\
3 \\
\end{array}$ & $\begin{array}{r}0.7 \\
8 \\
\end{array}$ & $\begin{array}{r}1519 . \\
5 \\
\end{array}$ & 16.9 & 1544.7 & 12.7 & $\begin{array}{r}1579 . \\
4 \\
\end{array}$ & 18.7 & 1579.4 & 18.7 & 96.2 \\
\hline $\mathrm{NBH} 4-33$ & 356 & 69820 & 1.6 & $\begin{array}{r}10.21 \\
80 \\
\end{array}$ & 1.7 & 3.7712 & 2.0 & $\begin{array}{r}0.279 \\
5 \\
\end{array}$ & $\begin{array}{l}1 . \\
0\end{array}$ & $\begin{array}{r}0.5 \\
0 \\
\end{array}$ & $\begin{array}{r}1588 . \\
7 \\
\end{array}$ & 14.1 & 1586.6 & 16.0 & $\begin{array}{r}1583 . \\
8 \\
\end{array}$ & 32.4 & 1583.8 & 32.4 & $\begin{array}{r}100 . \\
3 \\
\end{array}$ \\
\hline $\mathrm{NBH} 4-100$ & 767 & $\begin{array}{r}11926 \\
5 \\
\end{array}$ & 1.4 & $\begin{array}{r}10.21 \\
15 \\
\end{array}$ & 1.0 & 2.9837 & 2.6 & $\begin{array}{r}0.221 \\
0 \\
\end{array}$ & $\begin{array}{r}2 . \\
4 \\
\end{array}$ & $\begin{array}{r}0.9 \\
2 \\
\end{array}$ & $\begin{array}{r}1287 . \\
0 \\
\end{array}$ & 27.5 & 1403.5 & 19.5 & $\begin{array}{r}1585 . \\
0 \\
\end{array}$ & 18.7 & 1585.0 & 18.7 & 81.2 \\
\hline NBH4-71 & 256 & 79040 & 0.7 & $\begin{array}{r}10.11 \\
08 \\
\end{array}$ & 3.1 & 3.6643 & 3.4 & $\begin{array}{r}0.268 \\
7 \\
\end{array}$ & $\begin{array}{r}1 . \\
3 \\
\end{array}$ & $\begin{array}{r}0.3 \\
9 \\
\end{array}$ & $\begin{array}{r}1534 . \\
2 \\
\end{array}$ & 17.9 & 1563.6 & 26.8 & $\begin{array}{r}1603 . \\
5 \\
\end{array}$ & 57.6 & 1603.5 & 57.6 & 95.7 \\
\hline $\mathrm{NBH} 4-24$ & 411 & 84780 & 2.8 & $\begin{array}{r}10.09 \\
81 \\
\end{array}$ & 2.4 & 3.4369 & 3.0 & $\begin{array}{r}0.251 \\
7 \\
\end{array}$ & $\begin{array}{l}1 . \\
8\end{array}$ & $\begin{array}{r}0.6 \\
0 \\
\end{array}$ & $\begin{array}{r}1447 . \\
3 \\
\end{array}$ & 23.5 & 1512.9 & 23.7 & $\begin{array}{r}1605 . \\
9 \\
\end{array}$ & 44.9 & 1605.9 & 44.9 & 90.1 \\
\hline NBH4-6 & 762 & 97640 & 1.5 & $\begin{array}{r}10.08 \\
46 \\
\end{array}$ & 4.7 & 3.4924 & 5.0 & $\begin{array}{r}0.255 \\
4 \\
\end{array}$ & $\begin{array}{r}1 . \\
9 \\
\end{array}$ & $\begin{array}{r}0.3 \\
7 \\
\end{array}$ & $\begin{array}{r}1466 . \\
4 \\
\end{array}$ & 24.5 & 1525.5 & 39.7 & $\begin{array}{r}1608 . \\
4 \\
\end{array}$ & 86.9 & 1608.4 & 86.9 & 91.2 \\
\hline $\mathrm{NBH} 4-20$ & 265 & 59975 & 2.2 & 10.00 & 3.5 & 3.8398 & 3.6 & 0.278 & 1. & 0.2 & 1584. & 14.1 & 1601.1 & 29.2 & 1622. & 64.8 & 1622.6 & 64.8 & 97.7 \\
\hline
\end{tabular}




\begin{tabular}{|c|c|c|c|c|c|c|c|c|c|c|c|c|c|c|c|c|c|c|c|}
\hline & & & & 79 & & & & 7 & 0 & 8 & 9 & & & & 6 & & & & \\
\hline NBH4-51 & 255 & 55915 & 0.6 & $\begin{array}{r}10.00 \\
34\end{array}$ & 1.0 & 3.7020 & 1.4 & $\begin{array}{r}0.268 \\
6\end{array}$ & $\begin{array}{l}1 . \\
0\end{array}$ & $\begin{array}{r}0.7 \\
1\end{array}$ & $\begin{array}{r}1533 . \\
6\end{array}$ & 13.6 & 1571.8 & 11.3 & $\begin{array}{r}1623 . \\
4\end{array}$ & 18.6 & 1623.4 & 18.6 & 94.5 \\
\hline $\mathrm{NBH} 4-40$ & $\begin{array}{r}408 \\
1 \\
\end{array}$ & $\begin{array}{r}56790 \\
5 \\
\end{array}$ & 4.7 & $\begin{array}{r}9.884 \\
0 \\
\end{array}$ & 1.3 & 3.7505 & 1.6 & $\begin{array}{r}0.268 \\
9 \\
\end{array}$ & $\begin{array}{r}1 . \\
0\end{array}$ & $\begin{array}{r}0.6 \\
2 \\
\end{array}$ & $\begin{array}{r}1535 . \\
0\end{array}$ & 13.7 & 1582.2 & 13.0 & $\begin{array}{r}1645 . \\
7\end{array}$ & 23.7 & 1645.7 & 23.7 & 93.3 \\
\hline $\mathrm{NBH} 4-90$ & 441 & 70325 & 1.7 & $\begin{array}{r}9.852 \\
8\end{array}$ & 1.5 & 3.4176 & 2.7 & $\begin{array}{r}0.244 \\
2\end{array}$ & $\begin{array}{r}2 . \\
2\end{array}$ & $\begin{array}{r}0.8 \\
3\end{array}$ & $\begin{array}{r}1408 . \\
6\end{array}$ & 28.1 & 1508.4 & 21.0 & $\begin{array}{r}1651 . \\
6\end{array}$ & 27.4 & 1651.6 & 27.4 & 85.3 \\
\hline NBH4-86 & 770 & $\begin{array}{r}16904 \\
0 \\
\end{array}$ & 2.9 & $\begin{array}{r}9.819 \\
1 \\
\end{array}$ & 1.5 & 3.9500 & 2.0 & $\begin{array}{r}0.281 \\
3 \\
\end{array}$ & $\begin{array}{r}1 . \\
3\end{array}$ & $\begin{array}{r}0.6 \\
4 \\
\end{array}$ & $\begin{array}{r}1597 . \\
9 \\
\end{array}$ & 17.8 & 1624.0 & 16.0 & $\begin{array}{r}1658 . \\
0 \\
\end{array}$ & 28.2 & 1658.0 & 28.2 & 96.4 \\
\hline NBH4-7 & 186 & 39560 & 2.3 & $\begin{array}{r}9.810 \\
2\end{array}$ & 2.7 & 3.9051 & 4.0 & $\begin{array}{r}0.277 \\
8\end{array}$ & $\begin{array}{r}3 . \\
0\end{array}$ & $\begin{array}{r}0.7 \\
4\end{array}$ & $\begin{array}{r}1580 . \\
5\end{array}$ & 41.4 & 1614.7 & 32.1 & $\begin{array}{r}1659 . \\
6\end{array}$ & 49.3 & 1659.6 & 49.3 & 95.2 \\
\hline NBH4-84 & $\begin{array}{r}104 \\
7\end{array}$ & $\begin{array}{r}19477 \\
0\end{array}$ & 1.3 & $\begin{array}{r}9.804 \\
7\end{array}$ & 2.2 & 3.9071 & 2.4 & $\begin{array}{r}0.277 \\
8\end{array}$ & $\begin{array}{l}1 . \\
0\end{array}$ & $\begin{array}{r}0.4 \\
1\end{array}$ & $\begin{array}{r}1580 . \\
4\end{array}$ & 14.0 & 1615.2 & 19.8 & $\begin{array}{r}1660 . \\
7\end{array}$ & 41.3 & 1660.7 & 41.3 & 95.2 \\
\hline NBH4-94 & $\begin{array}{r}161 \\
1 \\
\end{array}$ & $\begin{array}{r}23840 \\
5\end{array}$ & 13.5 & $\begin{array}{r}9.737 \\
8\end{array}$ & 1.2 & 3.9436 & 1.6 & $\begin{array}{r}0.278 \\
5\end{array}$ & $\begin{array}{r}1 . \\
0\end{array}$ & $\begin{array}{r}0.6 \\
4\end{array}$ & $\begin{array}{r}1583 . \\
9\end{array}$ & 14.0 & 1622.7 & 12.6 & $\begin{array}{r}1673 . \\
3\end{array}$ & 22.0 & 1673.3 & 22.0 & 94.7 \\
\hline $\mathrm{NBH} 4-80$ & 718 & $\begin{array}{r}13493 \\
5 \\
\end{array}$ & 2.1 & $\begin{array}{r}9.725 \\
4 \\
\end{array}$ & 1.5 & 4.2122 & 1.8 & $\begin{array}{r}0.297 \\
1 \\
\end{array}$ & $\begin{array}{r}1 . \\
0\end{array}$ & $\begin{array}{r}0.5 \\
6 \\
\end{array}$ & $\begin{array}{r}1676 . \\
9 \\
\end{array}$ & 14.8 & 1676.4 & 14.6 & $\begin{array}{r}1675 . \\
7 \\
\end{array}$ & 27.2 & 1675.7 & 27.2 & $\begin{array}{r}100 . \\
1 \\
\end{array}$ \\
\hline NBH4-3 & 638 & $\begin{array}{r}11112 \\
0\end{array}$ & 1.5 & $\begin{array}{r}9.714 \\
7\end{array}$ & 5.5 & 4.2572 & 6.4 & $\begin{array}{r}0.299 \\
9\end{array}$ & $\begin{array}{r}3 . \\
3\end{array}$ & $\begin{array}{r}0.5 \\
1\end{array}$ & $\begin{array}{r}1691 . \\
1\end{array}$ & 48.8 & 1685.1 & 52.6 & $\begin{array}{r}1677 . \\
7\end{array}$ & $\begin{array}{r}101 . \\
3\end{array}$ & 1677.7 & $\begin{array}{r}101 . \\
3\end{array}$ & $\begin{array}{r}100 . \\
8\end{array}$ \\
\hline $\mathrm{NBH} 4-32$ & 537 & $\begin{array}{r}10722 \\
0 \\
\end{array}$ & 2.7 & $\begin{array}{r}9.640 \\
9 \\
\end{array}$ & 2.0 & 4.0634 & 3.0 & $\begin{array}{r}0.284 \\
1 \\
\end{array}$ & $\begin{array}{r}2 . \\
2 \\
\end{array}$ & $\begin{array}{r}0.7 \\
4 \\
\end{array}$ & $\begin{array}{r}1612 . \\
1 \\
\end{array}$ & 31.5 & 1647.0 & 24.3 & $\begin{array}{r}1691 . \\
8 \\
\end{array}$ & 37.1 & 1691.8 & 37.1 & 95.3 \\
\hline NBH4-78 & 617 & $\begin{array}{r}12069 \\
5\end{array}$ & 1.4 & $\begin{array}{r}9.624 \\
9\end{array}$ & 1.3 & 4.1655 & 1.8 & $\begin{array}{r}0.290 \\
8\end{array}$ & $\begin{array}{r}1 . \\
2\end{array}$ & $\begin{array}{r}0.6 \\
7\end{array}$ & $\begin{array}{r}1645 . \\
4\end{array}$ & 17.3 & 1667.3 & 14.6 & $\begin{array}{r}1694 . \\
9\end{array}$ & 24.5 & 1694.9 & 24.5 & 97.1 \\
\hline NBH4-98 & 581 & $\begin{array}{r}17402 \\
5 \\
\end{array}$ & 1.5 & $\begin{array}{r}9.623 \\
0 \\
\end{array}$ & 2.0 & 4.1033 & 3.0 & $\begin{array}{r}0.286 \\
4 \\
\end{array}$ & $\begin{array}{r}2 . \\
2 \\
\end{array}$ & $\begin{array}{r}0.7 \\
4 \\
\end{array}$ & $\begin{array}{r}1623 . \\
4 \\
\end{array}$ & 31.4 & 1655.0 & 24.2 & $\begin{array}{r}1695 . \\
2 \\
\end{array}$ & 36.9 & 1695.2 & 36.9 & 95.8 \\
\hline NBH4-96 & 465 & $\begin{array}{r}15071 \\
0 \\
\end{array}$ & 2.3 & $\begin{array}{r}9.590 \\
0 \\
\end{array}$ & 1.8 & 4.0383 & 3.2 & $\begin{array}{r}0.280 \\
9 \\
\end{array}$ & $\begin{array}{r}2 . \\
7\end{array}$ & $\begin{array}{r}0.8 \\
4 \\
\end{array}$ & $\begin{array}{r}1595 . \\
8\end{array}$ & 37.9 & 1641.9 & 26.1 & $\begin{array}{r}1701 . \\
6 \\
\end{array}$ & 32.2 & 1701.6 & 32.2 & 93.8 \\
\hline NBH4-8 & 315 & 59650 & 1.8 & $\begin{array}{r}9.588 \\
5\end{array}$ & 1.7 & 4.2324 & 2.4 & $\begin{array}{r}0.294 \\
3\end{array}$ & $\begin{array}{r}1 . \\
7\end{array}$ & $\begin{array}{r}0.7 \\
1\end{array}$ & $\begin{array}{r}1663 . \\
1\end{array}$ & 24.8 & 1680.3 & 19.5 & $\begin{array}{r}1701 . \\
8\end{array}$ & 30.6 & 1701.8 & 30.6 & 97.7 \\
\hline NBH4-76 & 684 & $\begin{array}{r}10751 \\
0 \\
\end{array}$ & 1.5 & $\begin{array}{r}9.575 \\
3 \\
\end{array}$ & 1.4 & 3.7321 & 2.0 & $\begin{array}{r}0.259 \\
2 \\
\end{array}$ & $\begin{array}{r}1 . \\
4\end{array}$ & $\begin{array}{r}0.7 \\
1 \\
\end{array}$ & $\begin{array}{r}1485 . \\
7\end{array}$ & 18.6 & 1578.3 & 15.8 & $\begin{array}{r}1704 . \\
4 \\
\end{array}$ & 25.6 & 1704.4 & 25.6 & 87.2 \\
\hline NBH4-97 & 532 & $\begin{array}{r}14015 \\
5\end{array}$ & 1.7 & $\begin{array}{r}9.550 \\
3\end{array}$ & 1.1 & 3.9686 & 2.3 & $\begin{array}{r}0.274 \\
9\end{array}$ & $\begin{array}{r}2 . \\
1\end{array}$ & $\begin{array}{r}0.8 \\
8\end{array}$ & $\begin{array}{r}1565 . \\
6\end{array}$ & 28.5 & 1627.8 & 19.0 & $\begin{array}{r}1709 . \\
2\end{array}$ & 20.8 & 1709.2 & 20.8 & 91.6 \\
\hline NBH4-31 & 344 & 78190 & 1.4 & $\begin{array}{r}9.548 \\
7 \\
\end{array}$ & 1.4 & 4.2469 & 2.2 & $\begin{array}{r}0.294 \\
1 \\
\end{array}$ & $\begin{array}{l}1 . \\
7 \\
\end{array}$ & $\begin{array}{r}0.7 \\
7 \\
\end{array}$ & $\begin{array}{r}1662 . \\
1 \\
\end{array}$ & 24.8 & 1683.1 & 18.1 & $\begin{array}{r}1709 . \\
5 \\
\end{array}$ & 25.9 & 1709.5 & 25.9 & 97.2 \\
\hline $\mathrm{NBH} 4-22$ & $\begin{array}{r}160 \\
0\end{array}$ & $\begin{array}{r}25982 \\
5\end{array}$ & 2.5 & $\begin{array}{r}9.543 \\
7\end{array}$ & 1.0 & 4.4370 & 1.4 & $\begin{array}{r}0.307 \\
1\end{array}$ & $\begin{array}{r}1 . \\
0\end{array}$ & $\begin{array}{r}0.7 \\
1\end{array}$ & $\begin{array}{r}1726 . \\
5\end{array}$ & 15.1 & 1719.3 & 11.7 & $\begin{array}{r}1710 . \\
5\end{array}$ & 18.4 & 1710.5 & 18.4 & $\begin{array}{r}100 . \\
9\end{array}$ \\
\hline $\mathrm{NBH} 4-42$ & $\begin{array}{r}205 \\
1\end{array}$ & 76520 & 7.0 & $\begin{array}{r}9.529 \\
6\end{array}$ & 4.0 & 3.4206 & 4.3 & $\begin{array}{r}0.236 \\
4\end{array}$ & $\begin{array}{r}1 . \\
6\end{array}$ & $\begin{array}{r}0.3 \\
7\end{array}$ & $\begin{array}{r}1368 . \\
0\end{array}$ & 19.4 & 1509.1 & 33.6 & $\begin{array}{r}1713 . \\
2\end{array}$ & 73.2 & 1713.2 & 73.2 & 79.9 \\
\hline NBH4-62 & $\begin{array}{r}121 \\
8\end{array}$ & $\begin{array}{r}16360 \\
5\end{array}$ & 0.8 & $\begin{array}{r}9.513 \\
0\end{array}$ & 2.0 & 4.1778 & 2.3 & $\begin{array}{r}0.288 \\
2\end{array}$ & $\begin{array}{r}1 . \\
0\end{array}$ & $\begin{array}{r}0.4 \\
4\end{array}$ & $\begin{array}{r}1632 . \\
8\end{array}$ & 14.4 & 1669.7 & 18.6 & $\begin{array}{r}1716 . \\
4\end{array}$ & 37.5 & 1716.4 & 37.5 & 95.1 \\
\hline NBH4-9 & 664 & $\begin{array}{r}17062 \\
0\end{array}$ & 8.5 & $\begin{array}{r}9.505 \\
8\end{array}$ & 2.3 & 4.3485 & 2.6 & $\begin{array}{r}0.299 \\
8\end{array}$ & $\begin{array}{r}1 . \\
1\end{array}$ & $\begin{array}{r}0.4 \\
1\end{array}$ & $\begin{array}{r}1690 . \\
3\end{array}$ & 15.6 & 1702.6 & 21.2 & $\begin{array}{r}1717 . \\
8\end{array}$ & 43.0 & 1717.8 & 43.0 & 98.4 \\
\hline $\mathrm{NBH} 4-53$ & $\begin{array}{r}158 \\
6\end{array}$ & $\begin{array}{r}24324 \\
5\end{array}$ & 3.1 & $\begin{array}{r}9.492 \\
9\end{array}$ & 7.7 & 4.0633 & 7.9 & $\begin{array}{r}0.279 \\
8\end{array}$ & $\begin{array}{r}1 . \\
6\end{array}$ & $\begin{array}{r}0.2 \\
1\end{array}$ & $\begin{array}{r}1590 . \\
1\end{array}$ & 23.0 & 1647.0 & 64.2 & $\begin{array}{r}1720 . \\
3\end{array}$ & $\begin{array}{r}141 . \\
7\end{array}$ & 1720.3 & $\begin{array}{r}141 . \\
7\end{array}$ & 92.4 \\
\hline NBH4-58 & 377 & 74420 & 2.5 & $\begin{array}{r}9.490 \\
2\end{array}$ & 1.4 & 4.1892 & 1.7 & $\begin{array}{r}0.288 \\
3\end{array}$ & $\begin{array}{r}1 . \\
0\end{array}$ & $\begin{array}{r}0.5 \\
9\end{array}$ & $\begin{array}{r}1633 . \\
2\end{array}$ & 14.4 & 1671.9 & 13.9 & $\begin{array}{r}1720 . \\
8\end{array}$ & 25.2 & 1720.8 & 25.2 & 94.9 \\
\hline NBH4-50 & $\begin{array}{r}135 \\
5\end{array}$ & $\begin{array}{r}28063 \\
5\end{array}$ & 1.6 & $\begin{array}{r}9.484 \\
0\end{array}$ & 1.2 & 4.0785 & 1.5 & $\begin{array}{r}0.280 \\
5\end{array}$ & $\begin{array}{l}1 . \\
0\end{array}$ & $\begin{array}{r}0.6 \\
5\end{array}$ & $\begin{array}{r}1594 . \\
1\end{array}$ & 14.1 & 1650.0 & 12.6 & $\begin{array}{r}1722 . \\
0\end{array}$ & 21.7 & 1722.0 & 21.7 & 92.6 \\
\hline
\end{tabular}




\begin{tabular}{|c|c|c|c|c|c|c|c|c|c|c|c|c|c|c|c|c|c|c|c|}
\hline $\mathrm{NBH} 4-49$ & 491 & $\begin{array}{r}10826 \\
0\end{array}$ & 1.6 & $\begin{array}{r}9.472 \\
6\end{array}$ & 1.5 & 4.1499 & 1.8 & $\begin{array}{r}0.285 \\
1\end{array}$ & $\begin{array}{l}1 . \\
0\end{array}$ & $\begin{array}{r}0.5 \\
6\end{array}$ & $\begin{array}{r}1617 . \\
0\end{array}$ & 14.3 & 1664.2 & 14.6 & $\begin{array}{r}1724 . \\
2\end{array}$ & 27.2 & 1724.2 & 27.2 & 93.8 \\
\hline $\mathrm{NBH} 4-27$ & $\begin{array}{r}171 \\
4\end{array}$ & $\begin{array}{r}23401 \\
5\end{array}$ & 1.3 & $\begin{array}{r}9.472 \\
2\end{array}$ & 1.7 & 4.4905 & 1.9 & $\begin{array}{r}0.308 \\
5\end{array}$ & $\begin{array}{l}1 . \\
0\end{array}$ & $\begin{array}{r}0.5 \\
2\end{array}$ & $\begin{array}{r}1733 . \\
3\end{array}$ & 15.2 & 1729.2 & 16.1 & $\begin{array}{r}1724 . \\
3\end{array}$ & 30.5 & 1724.3 & 30.5 & $\begin{array}{r}100 . \\
5\end{array}$ \\
\hline $\mathrm{NBH} 4-10$ & 256 & 61975 & 2.1 & $\begin{array}{r}9.462 \\
7 \\
\end{array}$ & 2.2 & 4.2614 & 4.0 & $\begin{array}{r}0.292 \\
5 \\
\end{array}$ & $\begin{array}{r}3 . \\
3 \\
\end{array}$ & $\begin{array}{r}0.8 \\
3\end{array}$ & $\begin{array}{r}1653 . \\
8\end{array}$ & 48.3 & 1685.9 & 32.7 & $\begin{array}{r}1726 . \\
1\end{array}$ & 40.6 & 1726.1 & 40.6 & 95.8 \\
\hline $\mathrm{NBH} 4-21$ & 565 & 90155 & 3.0 & $\begin{array}{r}9.440 \\
3\end{array}$ & 2.4 & 4.4086 & 2.8 & $\begin{array}{r}0.301 \\
8 \\
\end{array}$ & $\begin{array}{r}1 . \\
4 \\
\end{array}$ & $\begin{array}{r}0.4 \\
9 \\
\end{array}$ & $\begin{array}{r}1700 . \\
4\end{array}$ & 20.5 & 1714.0 & 23.1 & $\begin{array}{r}1730 . \\
5\end{array}$ & 44.6 & 1730.5 & 44.6 & 98.3 \\
\hline $\mathrm{NBH} 4-37$ & 865 & $\begin{array}{r}18787 \\
5\end{array}$ & 1.7 & $\begin{array}{r}9.425 \\
9\end{array}$ & 1.5 & 4.3934 & 2.3 & $\begin{array}{r}0.300 \\
3\end{array}$ & $\begin{array}{l}1 . \\
7\end{array}$ & $\begin{array}{r}0.7 \\
5\end{array}$ & $\begin{array}{r}1693 . \\
0\end{array}$ & 25.5 & 1711.1 & 18.9 & $\begin{array}{r}1733 . \\
3\end{array}$ & 27.9 & 1733.3 & 27.9 & 97.7 \\
\hline $\mathrm{NBH} 4-63$ & 908 & $\begin{array}{r}15344 \\
5 \\
\end{array}$ & 1.2 & $\begin{array}{r}9.418 \\
7\end{array}$ & 1.5 & 4.1365 & 2.2 & $\begin{array}{r}0.282 \\
6 \\
\end{array}$ & $\begin{array}{r}1 . \\
6\end{array}$ & $\begin{array}{r}0.7 \\
2 \\
\end{array}$ & $\begin{array}{r}1604 . \\
3\end{array}$ & 22.0 & 1661.5 & 17.7 & $\begin{array}{r}1734 . \\
7\end{array}$ & 27.7 & 1734.7 & 27.7 & 92.5 \\
\hline $\mathrm{NBH} 4-54$ & $\begin{array}{r}112 \\
3 \\
\end{array}$ & $\begin{array}{r}18855 \\
0\end{array}$ & 3.2 & $\begin{array}{r}9.412 \\
4\end{array}$ & 1.7 & 3.9987 & 4.1 & $\begin{array}{r}0.273 \\
0\end{array}$ & $\begin{array}{l}3 . \\
7\end{array}$ & $\begin{array}{r}0.9 \\
1\end{array}$ & $\begin{array}{r}1555 . \\
9\end{array}$ & 51.6 & 1633.9 & 33.2 & $\begin{array}{r}1735 . \\
9\end{array}$ & 30.6 & 1735.9 & 30.6 & 89.6 \\
\hline NBH4-99 & 157 & 44890 & 1.3 & $\begin{array}{r}9.408 \\
7\end{array}$ & 2.3 & 4.2224 & 3.5 & $\begin{array}{r}0.288 \\
1\end{array}$ & $\begin{array}{r}2 . \\
6\end{array}$ & $\begin{array}{r}0.7 \\
5\end{array}$ & $\begin{array}{r}1632 . \\
2\end{array}$ & 37.9 & 1678.4 & 28.7 & $\begin{array}{r}1736 . \\
6\end{array}$ & 42.2 & 1736.6 & 42.2 & 94.0 \\
\hline $\mathrm{NBH} 4-52$ & $\begin{array}{r}164 \\
8\end{array}$ & $\begin{array}{r}20091 \\
5\end{array}$ & 3.5 & $\begin{array}{r}9.393 \\
6\end{array}$ & 1.4 & 3.8699 & 2.3 & $\begin{array}{r}0.263 \\
7\end{array}$ & $\begin{array}{r}1 . \\
8\end{array}$ & $\begin{array}{r}0.7 \\
9\end{array}$ & $\begin{array}{r}1508 . \\
5\end{array}$ & 24.1 & 1607.4 & 18.3 & $\begin{array}{r}1739 . \\
6\end{array}$ & 25.7 & 1739.6 & 25.7 & 86.7 \\
\hline $\mathrm{NBH} 4-12$ & 347 & 99680 & 1.8 & $\begin{array}{r}9.388 \\
9\end{array}$ & 3.3 & 4.1692 & 3.7 & $\begin{array}{r}0.283 \\
9\end{array}$ & $\begin{array}{r}1 . \\
6\end{array}$ & $\begin{array}{r}0.4 \\
2\end{array}$ & $\begin{array}{r}1611 . \\
0\end{array}$ & 22.1 & 1668.0 & 29.9 & $\begin{array}{r}1740 . \\
5\end{array}$ & 60.7 & 1740.5 & 60.7 & 92.6 \\
\hline $\mathrm{NBH} 4-60$ & 277 & 31120 & 0.6 & $\begin{array}{r}9.357 \\
7\end{array}$ & 2.2 & 4.0978 & 2.7 & $\begin{array}{r}0.278 \\
1\end{array}$ & $\begin{array}{r}1 . \\
6\end{array}$ & $\begin{array}{r}0.5 \\
9\end{array}$ & $\begin{array}{r}1581 . \\
9\end{array}$ & 22.2 & 1653.9 & 22.0 & $\begin{array}{r}1746 . \\
6\end{array}$ & 40.0 & 1746.6 & 40.0 & 90.6 \\
\hline NBH4-1 & 662 & 62100 & 1.3 & $\begin{array}{r}9.339 \\
7\end{array}$ & 1.9 & 4.0788 & 2.6 & $\begin{array}{r}0.276 \\
3\end{array}$ & $\begin{array}{r}1 . \\
8\end{array}$ & $\begin{array}{r}0.6 \\
8\end{array}$ & $\begin{array}{r}1572 . \\
7\end{array}$ & 24.8 & 1650.1 & 21.2 & $\begin{array}{r}1750 . \\
1\end{array}$ & 34.8 & 1750.1 & 34.8 & 89.9 \\
\hline $\mathrm{NBH} 4-38$ & $\begin{array}{r}148 \\
8\end{array}$ & $\begin{array}{r}13317 \\
5\end{array}$ & 1.3 & $\begin{array}{r}9.084 \\
3\end{array}$ & 2.2 & 4.4609 & 2.6 & $\begin{array}{r}0.293 \\
9\end{array}$ & $\begin{array}{l}1 . \\
5\end{array}$ & $\begin{array}{r}0.5 \\
6\end{array}$ & $\begin{array}{r}1661 . \\
0\end{array}$ & 21.5 & 1723.7 & 21.6 & $\begin{array}{r}1800 . \\
7\end{array}$ & 39.1 & 1800.7 & 39.1 & 92.2 \\
\hline $\mathrm{NBH} 4-56$ & 320 & $\begin{array}{r}10972 \\
5\end{array}$ & 2.0 & $\begin{array}{r}8.940 \\
0\end{array}$ & 5.2 & 4.9278 & 6.0 & $\begin{array}{r}0.319 \\
5\end{array}$ & $\begin{array}{r}3 . \\
0\end{array}$ & $\begin{array}{r}0.5 \\
0\end{array}$ & $\begin{array}{r}1787 . \\
4\end{array}$ & 46.7 & 1807.0 & 50.7 & $\begin{array}{r}1829 . \\
8\end{array}$ & 94.5 & 1829.8 & 94.5 & 97.7 \\
\hline $\mathrm{NBH} 4-75$ & 702 & $\begin{array}{r}16771 \\
5\end{array}$ & 5.0 & $\begin{array}{r}8.883 \\
6\end{array}$ & 1.7 & 5.1457 & 2.4 & $\begin{array}{r}0.331 \\
5\end{array}$ & $\begin{array}{l}1 . \\
7\end{array}$ & $\begin{array}{r}0.7 \\
0\end{array}$ & $\begin{array}{r}1845 . \\
8\end{array}$ & 27.0 & 1843.7 & 20.5 & $\begin{array}{r}1841 . \\
3\end{array}$ & 31.3 & 1841.3 & 31.3 & $\begin{array}{r}100 . \\
2\end{array}$ \\
\hline NBH4-2 & 514 & 70590 & 2.8 & $\begin{array}{r}8.780 \\
0\end{array}$ & 2.5 & 5.1797 & 3.2 & $\begin{array}{r}0.329 \\
8\end{array}$ & $\begin{array}{r}2 . \\
1\end{array}$ & $\begin{array}{r}0.6 \\
5\end{array}$ & $\begin{array}{r}1837 . \\
6\end{array}$ & 33.1 & 1849.3 & 27.3 & $\begin{array}{r}1862 . \\
5\end{array}$ & 44.2 & 1862.5 & 44.2 & 98.7 \\
\hline NBH4-41 & 210 & 68515 & 0.6 & $\begin{array}{r}8.659 \\
0 \\
\end{array}$ & 2.1 & 5.1661 & 2.3 & $\begin{array}{r}0.324 \\
4 \\
\end{array}$ & $\begin{array}{r}1 . \\
0\end{array}$ & $\begin{array}{r}0.4 \\
3\end{array}$ & $\begin{array}{r}1811 . \\
4\end{array}$ & 15.8 & 1847.1 & 19.6 & $\begin{array}{r}1887 . \\
5\end{array}$ & 37.4 & 1887.5 & 37.4 & 96.0 \\
\hline $\mathrm{NBH} 4-25$ & 468 & 78845 & 3.8 & $\begin{array}{r}8.505 \\
1\end{array}$ & 2.5 & 5.2731 & 2.9 & $\begin{array}{r}0.325 \\
3\end{array}$ & $\begin{array}{l}1 . \\
4\end{array}$ & $\begin{array}{r}0.4 \\
9\end{array}$ & $\begin{array}{r}1815 . \\
4\end{array}$ & 22.6 & 1864.5 & 24.9 & $\begin{array}{r}1919 . \\
7\end{array}$ & 45.6 & 1919.7 & 45.6 & 94.6 \\
\hline NBH4-48 & $\begin{array}{r}100 \\
8\end{array}$ & $\begin{array}{r}13817 \\
5\end{array}$ & 1.5 & $\begin{array}{r}8.455 \\
7\end{array}$ & 1.7 & 4.6275 & 4.7 & $\begin{array}{r}0.283 \\
8\end{array}$ & $\begin{array}{r}4 . \\
4\end{array}$ & $\begin{array}{r}0.9 \\
3\end{array}$ & $\begin{array}{r}1610 . \\
4\end{array}$ & 62.0 & 1754.2 & 39.0 & $\begin{array}{r}1930 . \\
1\end{array}$ & 30.3 & 1930.1 & 30.3 & 83.4 \\
\hline $\mathrm{NBH} 4-26$ & 124 & 37060 & 0.9 & $\begin{array}{r}8.365 \\
7\end{array}$ & 2.5 & 5.8750 & 2.7 & $\begin{array}{r}0.356 \\
5\end{array}$ & $\begin{array}{r}1 . \\
0\end{array}$ & $\begin{array}{r}0.3 \\
7\end{array}$ & $\begin{array}{r}1965 . \\
4\end{array}$ & 16.9 & 1957.6 & 23.3 & $\begin{array}{r}1949 . \\
3\end{array}$ & 44.5 & 1949.3 & 44.5 & $\begin{array}{r}100 . \\
8\end{array}$ \\
\hline $\mathrm{NBH} 4-72$ & 334 & $\begin{array}{r}13392 \\
5\end{array}$ & 1.5 & $\begin{array}{r}8.052 \\
8\end{array}$ & 3.9 & 6.0161 & 6.4 & $\begin{array}{r}0.351 \\
4\end{array}$ & $\begin{array}{r}5 . \\
1\end{array}$ & $\begin{array}{r}0.7 \\
9\end{array}$ & $\begin{array}{r}1941 . \\
1\end{array}$ & 84.6 & 1978.2 & 55.7 & $\begin{array}{r}2017 . \\
1\end{array}$ & 69.3 & 2017.1 & 69.3 & 96.2 \\
\hline NBH4-16 & 280 & $\begin{array}{r}12001 \\
5\end{array}$ & 2.3 & $\begin{array}{r}6.709 \\
0\end{array}$ & 1.2 & 8.6967 & 1.6 & $\begin{array}{r}0.423 \\
2\end{array}$ & $\begin{array}{r}1 . \\
0\end{array}$ & $\begin{array}{r}0.6 \\
4\end{array}$ & $\begin{array}{r}2274 . \\
8\end{array}$ & 19.2 & 2306.7 & 14.3 & $\begin{array}{r}2335 . \\
1\end{array}$ & 20.7 & 2335.1 & 20.7 & 97.4 \\
\hline NBH4-61 & $\begin{array}{r}174 \\
4 \\
\end{array}$ & $\begin{array}{r}44324 \\
5\end{array}$ & 5.7 & $\begin{array}{r}6.450 \\
9\end{array}$ & 4.0 & 9.0623 & 4.2 & $\begin{array}{r}0.424 \\
0\end{array}$ & $\begin{array}{r}1 . \\
0\end{array}$ & $\begin{array}{r}0.2 \\
4\end{array}$ & $\begin{array}{r}2278 . \\
6\end{array}$ & 19.2 & 2344.3 & 38.1 & $\begin{array}{r}2402 . \\
0\end{array}$ & 68.7 & 2402.0 & 68.7 & 94.9 \\
\hline $\mathrm{NBH} 4-77$ & 867 & $\begin{array}{r}18726 \\
0\end{array}$ & 1.1 & $\begin{array}{r}6.189 \\
3\end{array}$ & 1.7 & 9.7005 & 3.0 & $\begin{array}{r}0.435 \\
4 \\
\end{array}$ & $\begin{array}{r}2 . \\
5\end{array}$ & $\begin{array}{r}0.8 \\
4 \\
\end{array}$ & $\begin{array}{r}2330 . \\
2\end{array}$ & 49.1 & 2406.8 & 27.7 & $\begin{array}{r}2472 . \\
1\end{array}$ & 27.9 & 2472.1 & 27.9 & 94.3 \\
\hline $\mathrm{NBH} 4-11$ & 163 & 77230 & 2.4 & 5.395 & 3.7 & 13.551 & 3.9 & 0.530 & 1. & 0.2 & 2742. & 23.2 & 2718.9 & 36.5 & 2701. & 61.4 & 2701.4 & 61.4 & 101. \\
\hline
\end{tabular}




\begin{tabular}{|c|c|c|c|c|c|c|c|c|c|c|c|c|c|c|c|c|c|c|c|}
\hline & & & & 0 & & 1 & & 2 & 0 & 7 & 4 & & & & 4 & & & & 5 \\
\hline $\mathrm{NBH} 4-30$ & 105 & 75400 & 1.7 & $\begin{array}{r}2.976 \\
2 \\
\end{array}$ & 2.5 & $\begin{array}{r}35.481 \\
4 \\
\end{array}$ & 2.7 & $\begin{array}{r}0.765 \\
9 \\
\end{array}$ & $\begin{array}{r}1 . \\
0\end{array}$ & $\begin{array}{r}0.3 \\
7 \\
\end{array}$ & $\begin{array}{r}3665 . \\
8 \\
\end{array}$ & 28.0 & 3652.1 & 26.6 & $\begin{array}{r}3644 . \\
7 \\
\end{array}$ & 38.3 & 3644.7 & 38.3 & $\begin{array}{r}100 . \\
6 \\
\end{array}$ \\
\hline NBH8-38 & 237 & $\begin{array}{r}12416 \\
0 \\
\end{array}$ & 2.0 & $\begin{array}{r}9.165 \\
5 \\
\end{array}$ & 3.8 & 4.9392 & 3.9 & $\begin{array}{r}0.328 \\
3 \\
\end{array}$ & $\begin{array}{r}1 . \\
0 \\
\end{array}$ & $\begin{array}{r}0.2 \\
6 \\
\end{array}$ & $\begin{array}{r}1830 . \\
3 \\
\end{array}$ & 15.9 & 1809.0 & 33.0 & $\begin{array}{r}1784 . \\
5 \\
\end{array}$ & 68.9 & 1784.5 & 68.9 & $\begin{array}{r}102 . \\
6 \\
\end{array}$ \\
\hline NBH8-51 & $\begin{array}{r}172 \\
7\end{array}$ & $\begin{array}{r}44127 \\
0\end{array}$ & 1.2 & $\begin{array}{r}9.141 \\
3\end{array}$ & 4.7 & 4.6855 & 4.9 & $\begin{array}{r}0.310 \\
6\end{array}$ & $\begin{array}{r}1 . \\
4\end{array}$ & $\begin{array}{r}0.2 \\
8\end{array}$ & $\begin{array}{r}1743 . \\
9\end{array}$ & 21.2 & 1764.6 & 41.3 & $\begin{array}{r}1789 . \\
3\end{array}$ & 86.2 & 1789.3 & 86.2 & 97.5 \\
\hline NBH8-28 & 655 & $\begin{array}{r}12211 \\
0\end{array}$ & 4.1 & $\begin{array}{r}9.059 \\
5\end{array}$ & 2.5 & 4.7945 & 2.7 & $\begin{array}{r}0.315 \\
0\end{array}$ & $\begin{array}{r}1 . \\
0\end{array}$ & $\begin{array}{r}0.3 \\
7\end{array}$ & $\begin{array}{r}1765 . \\
4\end{array}$ & 15.4 & 1783.9 & 22.5 & $\begin{array}{r}1805 . \\
7\end{array}$ & 45.1 & 1805.7 & 45.1 & 97.8 \\
\hline NBH8-26 & 579 & $\begin{array}{r}16821 \\
0\end{array}$ & 5.8 & $\begin{array}{r}9.032 \\
5 \\
\end{array}$ & 4.1 & 4.7157 & 4.3 & $\begin{array}{r}0.308 \\
9 \\
\end{array}$ & $\begin{array}{r}1 . \\
2\end{array}$ & $\begin{array}{r}0.2 \\
7 \\
\end{array}$ & $\begin{array}{r}1735 . \\
4\end{array}$ & 17.8 & 1770.0 & 36.0 & $\begin{array}{r}1811 . \\
1\end{array}$ & 75.1 & 1811.1 & 75.1 & 95.8 \\
\hline NBH8-18 & 990 & $\begin{array}{r}11752 \\
0\end{array}$ & 5.1 & $\begin{array}{r}8.990 \\
9\end{array}$ & 2.7 & 3.7740 & 3.9 & $\begin{array}{r}0.246 \\
1\end{array}$ & $\begin{array}{r}2 . \\
8\end{array}$ & $\begin{array}{r}0.7 \\
1\end{array}$ & $\begin{array}{r}1418 . \\
3\end{array}$ & 35.6 & 1587.2 & 31.5 & $\begin{array}{r}1819 . \\
5\end{array}$ & 49.7 & 1819.5 & 49.7 & 78.0 \\
\hline NBH8-36 & $\begin{array}{r}149 \\
1\end{array}$ & $\begin{array}{r}22338 \\
0\end{array}$ & 3.7 & $\begin{array}{r}8.969 \\
1\end{array}$ & 4.8 & 4.8605 & 5.4 & $\begin{array}{r}0.316 \\
2\end{array}$ & $\begin{array}{r}2 . \\
4\end{array}$ & $\begin{array}{r}0.4 \\
4\end{array}$ & $\begin{array}{r}1771 . \\
0\end{array}$ & 37.2 & 1795.4 & 45.4 & $\begin{array}{r}1823 . \\
9\end{array}$ & 87.7 & 1823.9 & 87.7 & 97.1 \\
\hline NBH8-17 & $\begin{array}{r}123 \\
9 \\
\end{array}$ & $\begin{array}{r}36142 \\
0\end{array}$ & 1.9 & $\begin{array}{r}8.929 \\
9\end{array}$ & 3.2 & 4.5197 & 3.8 & $\begin{array}{r}0.292 \\
7\end{array}$ & $\begin{array}{r}2 . \\
0\end{array}$ & $\begin{array}{r}0.5 \\
3\end{array}$ & $\begin{array}{r}1655 . \\
1\end{array}$ & 29.0 & 1734.6 & 31.4 & $\begin{array}{r}1831 . \\
8\end{array}$ & 58.2 & 1831.8 & 58.2 & 90.4 \\
\hline NBH8-42 & 454 & $\begin{array}{r}27063 \\
5\end{array}$ & 6.5 & $\begin{array}{r}8.897 \\
8\end{array}$ & 2.5 & 4.9041 & 2.7 & $\begin{array}{r}0.316 \\
5\end{array}$ & $\begin{array}{r}1 . \\
0\end{array}$ & $\begin{array}{r}0.3 \\
7\end{array}$ & $\begin{array}{r}1772 . \\
5\end{array}$ & 15.5 & 1803.0 & 22.9 & $\begin{array}{r}1838 . \\
4\end{array}$ & 45.8 & 1838.4 & 45.8 & 96.4 \\
\hline $\mathrm{NBH} 8-25$ & 281 & $\begin{array}{r}10826 \\
5 \\
\end{array}$ & 1.6 & $\begin{array}{r}8.890 \\
5 \\
\end{array}$ & 5.0 & 5.2817 & 5.1 & $\begin{array}{r}0.340 \\
6\end{array}$ & $\begin{array}{r}1 . \\
0\end{array}$ & $\begin{array}{r}0.2 \\
0\end{array}$ & $\begin{array}{r}1889 . \\
4\end{array}$ & 16.4 & 1865.9 & 43.6 & $\begin{array}{r}1839 . \\
8\end{array}$ & 90.8 & 1839.8 & 90.8 & $\begin{array}{r}102 . \\
7\end{array}$ \\
\hline NBH8-9 & 309 & $\begin{array}{r}17448 \\
0\end{array}$ & 3.9 & $\begin{array}{r}8.874 \\
9 \\
\end{array}$ & 4.3 & 5.2145 & 4.4 & $\begin{array}{r}0.335 \\
6 \\
\end{array}$ & $\begin{array}{r}1 . \\
0\end{array}$ & $\begin{array}{r}0.2 \\
3 \\
\end{array}$ & $\begin{array}{r}1865 . \\
7\end{array}$ & 16.2 & 1855.0 & 37.6 & $\begin{array}{r}1843 . \\
0\end{array}$ & 77.9 & 1843.0 & 77.9 & $\begin{array}{r}101 . \\
2 \\
\end{array}$ \\
\hline NBH8-29 & 768 & $\begin{array}{r}18719 \\
5\end{array}$ & 1.7 & $\begin{array}{r}8.863 \\
7\end{array}$ & 1.8 & 5.1194 & 2.1 & $\begin{array}{r}0.329 \\
1\end{array}$ & $\begin{array}{r}1 . \\
0\end{array}$ & $\begin{array}{r}0.4 \\
8\end{array}$ & $\begin{array}{r}1834 . \\
0\end{array}$ & 16.0 & 1839.3 & 17.8 & $\begin{array}{r}1845 . \\
3\end{array}$ & 33.3 & 1845.3 & 33.3 & 99.4 \\
\hline NBH8-49 & 457 & $\begin{array}{r}10515 \\
0\end{array}$ & 2.3 & $\begin{array}{r}8.857 \\
7\end{array}$ & 3.5 & 4.7870 & 3.6 & $\begin{array}{r}0.307 \\
5\end{array}$ & $\begin{array}{r}1 . \\
0\end{array}$ & $\begin{array}{r}0.2 \\
8\end{array}$ & $\begin{array}{r}1728 . \\
5\end{array}$ & 15.2 & 1782.6 & 30.4 & $\begin{array}{r}1846 . \\
5\end{array}$ & 63.0 & 1846.5 & 63.0 & 93.6 \\
\hline NBH8-34 & 798 & $\begin{array}{r}10429 \\
5\end{array}$ & 1.6 & $\begin{array}{r}8.831 \\
5\end{array}$ & 2.4 & 4.6662 & 2.8 & $\begin{array}{r}0.298 \\
9\end{array}$ & $\begin{array}{l}1 . \\
5\end{array}$ & $\begin{array}{r}0.5 \\
2\end{array}$ & $\begin{array}{r}1685 . \\
8\end{array}$ & 21.5 & 1761.2 & 23.5 & $\begin{array}{r}1851 . \\
9\end{array}$ & 43.6 & 1851.9 & 43.6 & 91.0 \\
\hline NBH8-37 & 532 & $\begin{array}{r}24230 \\
5\end{array}$ & 1.7 & $\begin{array}{r}8.823 \\
8\end{array}$ & 2.7 & 5.3208 & 2.8 & $\begin{array}{r}0.340 \\
5\end{array}$ & $\begin{array}{r}1 . \\
0\end{array}$ & $\begin{array}{r}0.3 \\
5\end{array}$ & $\begin{array}{r}1889 . \\
1\end{array}$ & 16.4 & 1872.2 & 24.3 & $\begin{array}{r}1853 . \\
5\end{array}$ & 48.1 & 1853.5 & 48.1 & $\begin{array}{r}101 . \\
9\end{array}$ \\
\hline NBH8-19 & 263 & $\begin{array}{r}14983 \\
0\end{array}$ & 3.1 & $\begin{array}{r}8.803 \\
5 \\
\end{array}$ & 3.2 & 5.2153 & 3.3 & $\begin{array}{r}0.333 \\
0 \\
\end{array}$ & $\begin{array}{r}1 . \\
1\end{array}$ & $\begin{array}{r}0.3 \\
2 \\
\end{array}$ & $\begin{array}{r}1852 . \\
9\end{array}$ & 17.1 & 1855.1 & 28.4 & $\begin{array}{r}1857 . \\
6\end{array}$ & 57.1 & 1857.6 & 57.1 & 99.7 \\
\hline NBH8-59 & 278 & 91440 & 3.3 & $\begin{array}{r}8.791 \\
9\end{array}$ & 2.5 & 5.3327 & 2.8 & $\begin{array}{r}0.340 \\
0\end{array}$ & $\begin{array}{r}1 . \\
2\end{array}$ & $\begin{array}{r}0.4 \\
3\end{array}$ & $\begin{array}{r}1886 . \\
9\end{array}$ & 19.3 & 1874.1 & 23.7 & $\begin{array}{r}1860 . \\
0\end{array}$ & 45.3 & 1860.0 & 45.3 & $\begin{array}{r}101 . \\
4\end{array}$ \\
\hline NBH8-44 & 700 & $\begin{array}{r}29697 \\
5\end{array}$ & 0.7 & $\begin{array}{r}8.782 \\
5\end{array}$ & 3.2 & 5.2010 & 3.4 & $\begin{array}{r}0.331 \\
3\end{array}$ & $\begin{array}{r}1 . \\
2\end{array}$ & $\begin{array}{r}0.3 \\
4\end{array}$ & $\begin{array}{r}1844 . \\
6\end{array}$ & 18.4 & 1852.8 & 28.6 & $\begin{array}{r}1861 . \\
9\end{array}$ & 56.9 & 1861.9 & 56.9 & 99.1 \\
\hline NBH8-8 & 548 & $\begin{array}{r}12531 \\
0\end{array}$ & 2.4 & $\begin{array}{r}8.780 \\
7\end{array}$ & 3.7 & 4.8958 & 4.8 & $\begin{array}{r}0.311 \\
8\end{array}$ & $\begin{array}{r}3 . \\
0\end{array}$ & $\begin{array}{r}0.6 \\
3\end{array}$ & $\begin{array}{r}1749 . \\
5\end{array}$ & 45.7 & 1801.5 & 40.1 & $\begin{array}{r}1862 . \\
3\end{array}$ & 66.8 & 1862.3 & 66.8 & 93.9 \\
\hline NBH8-80 & 375 & $\begin{array}{r}27711 \\
5 \\
\end{array}$ & 1.1 & $\begin{array}{r}8.775 \\
2 \\
\end{array}$ & 4.7 & 5.2986 & 5.4 & $\begin{array}{r}0.337 \\
2 \\
\end{array}$ & $\begin{array}{r}2 . \\
7 \\
\end{array}$ & $\begin{array}{r}0.5 \\
0 \\
\end{array}$ & $\begin{array}{r}1873 . \\
3 \\
\end{array}$ & 43.7 & 1868.6 & 46.4 & $\begin{array}{r}1863 . \\
4 \\
\end{array}$ & 85.1 & 1863.4 & 85.1 & $\begin{array}{r}100 . \\
5 \\
\end{array}$ \\
\hline NBH8-16 & 371 & $\begin{array}{r}13705 \\
5\end{array}$ & 2.6 & $\begin{array}{r}8.761 \\
1\end{array}$ & 4.3 & 5.3389 & 4.4 & $\begin{array}{r}0.339 \\
2\end{array}$ & $\begin{array}{r}1 . \\
0\end{array}$ & $\begin{array}{r}0.2 \\
3\end{array}$ & $\begin{array}{r}1883 . \\
0\end{array}$ & 16.3 & 1875.1 & 37.8 & $\begin{array}{r}1866 . \\
3\end{array}$ & 77.6 & 1866.3 & 77.6 & $\begin{array}{r}100 . \\
9\end{array}$ \\
\hline NBH8-61 & 331 & $\begin{array}{r}21358 \\
5\end{array}$ & 1.7 & $\begin{array}{r}8.717 \\
7\end{array}$ & 4.8 & 5.3967 & 5.0 & $\begin{array}{r}0.341 \\
2\end{array}$ & $\begin{array}{r}1 . \\
5\end{array}$ & $\begin{array}{r}0.2 \\
9\end{array}$ & $\begin{array}{r}1892 . \\
5\end{array}$ & 23.8 & 1884.3 & 42.6 & $\begin{array}{r}1875 . \\
3\end{array}$ & 85.7 & 1875.3 & 85.7 & $\begin{array}{r}100 . \\
9\end{array}$ \\
\hline NBH8-56 & 533 & $\begin{array}{r}30363 \\
0\end{array}$ & 2.2 & $\begin{array}{r}8.702 \\
1\end{array}$ & 1.9 & 5.0532 & 2.7 & $\begin{array}{r}0.318 \\
9\end{array}$ & $\begin{array}{r}2 . \\
0\end{array}$ & $\begin{array}{r}0.7 \\
3\end{array}$ & $\begin{array}{r}1784 . \\
5\end{array}$ & 31.2 & 1828.3 & 23.2 & $\begin{array}{r}1878 . \\
5\end{array}$ & 33.7 & 1878.5 & 33.7 & 95.0 \\
\hline
\end{tabular}




\begin{tabular}{|c|c|c|c|c|c|c|c|c|c|c|c|c|c|c|c|c|c|c|c|}
\hline NBH8-66 & 113 & 63120 & 1.2 & $\begin{array}{r}8.674 \\
4\end{array}$ & 3.7 & 5.5074 & 4.3 & $\begin{array}{r}0.346 \\
5\end{array}$ & $\begin{array}{r}2 . \\
3\end{array}$ & $\begin{array}{r}0.5 \\
4\end{array}$ & $\begin{array}{r}1917 . \\
8\end{array}$ & 38.8 & 1901.8 & 37.3 & $\begin{array}{r}1884 . \\
3\end{array}$ & 65.8 & 1884.3 & 65.8 & $\begin{array}{r}101 . \\
8\end{array}$ \\
\hline NBH8-32 & 298 & $\begin{array}{r}12868 \\
0\end{array}$ & 4.2 & $\begin{array}{r}8.657 \\
3\end{array}$ & 2.8 & 5.3556 & 3.1 & $\begin{array}{r}0.336 \\
3\end{array}$ & $\begin{array}{r}1 . \\
4\end{array}$ & $\begin{array}{r}0.4 \\
3\end{array}$ & $\begin{array}{r}1868 . \\
7\end{array}$ & 21.9 & 1877.8 & 26.8 & $\begin{array}{r}1887 . \\
8\end{array}$ & 51.0 & 1887.8 & 51.0 & 99.0 \\
\hline NBH8-4 & 198 & $\begin{array}{r}10981 \\
5\end{array}$ & 1.5 & $\begin{array}{r}8.624 \\
5\end{array}$ & 3.4 & 5.5056 & 3.6 & $\begin{array}{r}0.344 \\
4\end{array}$ & $\begin{array}{r}1 . \\
3\end{array}$ & $\begin{array}{r}0.3 \\
7\end{array}$ & $\begin{array}{r}1907 . \\
7\end{array}$ & 22.1 & 1901.5 & 31.0 & $\begin{array}{r}1894 . \\
7\end{array}$ & 60.3 & 1894.7 & 60.3 & $\begin{array}{r}100 . \\
7\end{array}$ \\
\hline NBH8-78 & 492 & $\begin{array}{r}21364 \\
5 \\
\end{array}$ & 2.2 & $\begin{array}{r}8.572 \\
1 \\
\end{array}$ & 3.6 & 5.3411 & 3.8 & $\begin{array}{r}0.332 \\
1 \\
\end{array}$ & $\begin{array}{r}1 . \\
1\end{array}$ & $\begin{array}{r}0.2 \\
9 \\
\end{array}$ & $\begin{array}{r}1848 . \\
4 \\
\end{array}$ & 17.7 & 1875.5 & 32.5 & $\begin{array}{r}1905 . \\
6 \\
\end{array}$ & 65.4 & 1905.6 & 65.4 & 97.0 \\
\hline NBH8-14 & 310 & 97630 & 3.7 & $\begin{array}{r}8.560 \\
1 \\
\end{array}$ & 2.4 & 5.6364 & 2.6 & $\begin{array}{r}0.349 \\
9 \\
\end{array}$ & $\begin{array}{r}1 . \\
0\end{array}$ & $\begin{array}{r}0.3 \\
8 \\
\end{array}$ & $\begin{array}{r}1934 . \\
3\end{array}$ & 16.7 & 1921.7 & 22.6 & $\begin{array}{r}1908 . \\
1\end{array}$ & 43.5 & 1908.1 & 43.5 & $\begin{array}{r}101 . \\
4 \\
\end{array}$ \\
\hline $\mathrm{NBH} 8-64$ & 630 & $\begin{array}{r}34807 \\
0 \\
\end{array}$ & 6.9 & $\begin{array}{r}8.557 \\
2 \\
\end{array}$ & 4.4 & 5.2554 & 4.5 & $\begin{array}{r}0.326 \\
2 \\
\end{array}$ & $\begin{array}{r}1 . \\
0\end{array}$ & $\begin{array}{r}0.2 \\
2 \\
\end{array}$ & $\begin{array}{r}1819 . \\
8 \\
\end{array}$ & 15.9 & 1861.7 & 38.3 & $\begin{array}{r}1908 . \\
7 \\
\end{array}$ & 78.7 & 1908.7 & 78.7 & 95.3 \\
\hline NBH8-58 & 514 & $\begin{array}{r}24521 \\
0\end{array}$ & 2.8 & $\begin{array}{r}8.554 \\
9\end{array}$ & 2.9 & 5.3692 & 3.1 & $\begin{array}{r}0.333 \\
1\end{array}$ & $\begin{array}{r}1 . \\
0\end{array}$ & $\begin{array}{r}0.3 \\
3\end{array}$ & $\begin{array}{r}1853 . \\
6\end{array}$ & 16.1 & 1880.0 & 26.2 & $\begin{array}{r}1909 . \\
2\end{array}$ & 51.9 & 1909.2 & 51.9 & 97.1 \\
\hline $\mathrm{NBH} 8-43$ & 418 & $\begin{array}{r}26690 \\
5 \\
\end{array}$ & 2.6 & $\begin{array}{r}8.549 \\
9 \\
\end{array}$ & 3.1 & 5.4087 & 3.6 & $\begin{array}{r}0.335 \\
4 \\
\end{array}$ & $\begin{array}{r}1 . \\
9 \\
\end{array}$ & $\begin{array}{r}0.5 \\
3 \\
\end{array}$ & $\begin{array}{r}1864 . \\
5 \\
\end{array}$ & 30.9 & 1886.2 & 31.1 & $\begin{array}{r}1910 . \\
3 \\
\end{array}$ & 55.3 & 1910.3 & 55.3 & 97.6 \\
\hline NBH8-39 & 676 & $\begin{array}{r}29912 \\
0 \\
\end{array}$ & 24.8 & $\begin{array}{r}8.546 \\
6 \\
\end{array}$ & 2.9 & 5.3889 & 3.2 & $\begin{array}{r}0.334 \\
0 \\
\end{array}$ & $\begin{array}{r}1 . \\
3\end{array}$ & $\begin{array}{r}0.4 \\
2 \\
\end{array}$ & $\begin{array}{r}1857 . \\
9 \\
\end{array}$ & 21.6 & 1883.1 & 27.5 & $\begin{array}{r}1911 . \\
0\end{array}$ & 52.4 & 1911.0 & 52.4 & 97.2 \\
\hline NBH8-31 & 896 & $\begin{array}{r}13501 \\
5\end{array}$ & 4.4 & $\begin{array}{r}8.534 \\
0\end{array}$ & 1.8 & 4.5707 & 5.6 & $\begin{array}{r}0.282 \\
9\end{array}$ & $\begin{array}{r}5 . \\
3\end{array}$ & $\begin{array}{r}0.9 \\
5\end{array}$ & $\begin{array}{r}1605 . \\
9\end{array}$ & 75.9 & 1743.9 & 46.9 & $\begin{array}{r}1913 . \\
6\end{array}$ & 31.6 & 1913.6 & 31.6 & 83.9 \\
\hline $\mathrm{NBH} 8-63$ & 223 & $\begin{array}{r}15237 \\
5 \\
\end{array}$ & 1.6 & $\begin{array}{r}8.524 \\
0 \\
\end{array}$ & 2.6 & 5.7412 & 2.8 & $\begin{array}{r}0.354 \\
9 \\
\end{array}$ & $\begin{array}{r}1 . \\
0 \\
\end{array}$ & $\begin{array}{r}0.3 \\
6 \\
\end{array}$ & $\begin{array}{r}1958 . \\
1 \\
\end{array}$ & 16.9 & 1937.6 & 24.3 & $\begin{array}{r}1915 . \\
7 \\
\end{array}$ & 47.0 & 1915.7 & 47.0 & $\begin{array}{r}102 . \\
2 \\
\end{array}$ \\
\hline NBH8-55 & 393 & $\begin{array}{r}24016 \\
0\end{array}$ & 2.7 & $\begin{array}{r}8.522 \\
8\end{array}$ & 4.2 & 5.8144 & 4.3 & $\begin{array}{r}0.359 \\
4\end{array}$ & $\begin{array}{r}1 . \\
0\end{array}$ & $\begin{array}{r}0.2 \\
3\end{array}$ & $\begin{array}{r}1979 . \\
3\end{array}$ & 17.0 & 1948.6 & 37.3 & $\begin{array}{r}1916 . \\
0\end{array}$ & 75.2 & 1916.0 & 75.2 & $\begin{array}{r}103 . \\
3\end{array}$ \\
\hline NBH8-65 & $\begin{array}{r}118 \\
5 \\
\end{array}$ & $\begin{array}{r}44342 \\
5 \\
\end{array}$ & 11.0 & $\begin{array}{r}8.507 \\
6 \\
\end{array}$ & 3.2 & 5.4791 & 3.4 & $\begin{array}{r}0.338 \\
1 \\
\end{array}$ & $\begin{array}{r}1 . \\
0 \\
\end{array}$ & $\begin{array}{r}0.3 \\
0 \\
\end{array}$ & $\begin{array}{r}1877 . \\
4 \\
\end{array}$ & 16.3 & 1897.3 & 28.8 & $\begin{array}{r}1919 . \\
2 \\
\end{array}$ & 57.4 & 1919.2 & 57.4 & 97.8 \\
\hline NBH8-15 & 632 & $\begin{array}{r}18312 \\
5\end{array}$ & 1.7 & $\begin{array}{r}8.490 \\
4\end{array}$ & 5.5 & 5.2525 & 5.9 & $\begin{array}{r}0.323 \\
4\end{array}$ & $\begin{array}{r}2 . \\
3\end{array}$ & $\begin{array}{r}0.3 \\
9\end{array}$ & $\begin{array}{r}1806 . \\
5\end{array}$ & 36.2 & 1861.2 & 50.6 & $\begin{array}{r}1922 . \\
8\end{array}$ & 98.0 & 1922.8 & 98.0 & 94.0 \\
\hline NBH8-24 & $\begin{array}{r}154 \\
8 \\
\end{array}$ & $\begin{array}{r}25567 \\
5\end{array}$ & 6.3 & $\begin{array}{r}8.472 \\
2\end{array}$ & 2.4 & 4.5323 & 2.6 & $\begin{array}{r}0.278 \\
5\end{array}$ & $\begin{array}{r}1 . \\
0\end{array}$ & $\begin{array}{r}0.3 \\
8\end{array}$ & $\begin{array}{r}1583 . \\
8\end{array}$ & 14.0 & 1736.9 & 21.9 & $\begin{array}{r}1926 . \\
6\end{array}$ & 43.5 & 1926.6 & 43.5 & 82.2 \\
\hline NBH8-7 & 117 & 13840 & 2.9 & $\begin{array}{r}8.464 \\
4 \\
\end{array}$ & 1.8 & 4.3497 & 7.2 & $\begin{array}{r}0.267 \\
0\end{array}$ & $\begin{array}{r}7 . \\
0\end{array}$ & $\begin{array}{r}0.9 \\
7 \\
\end{array}$ & $\begin{array}{r}1525 . \\
7\end{array}$ & 94.7 & 1702.8 & 59.5 & $\begin{array}{r}1928 . \\
3\end{array}$ & 32.1 & 1928.3 & 32.1 & 79.1 \\
\hline NBH8-60 & 526 & $\begin{array}{r}18732 \\
0 \\
\end{array}$ & 6.7 & $\begin{array}{r}8.461 \\
8 \\
\end{array}$ & 2.7 & 5.2679 & 3.4 & $\begin{array}{r}0.323 \\
3 \\
\end{array}$ & $\begin{array}{r}2 . \\
0 \\
\end{array}$ & $\begin{array}{r}0.5 \\
9 \\
\end{array}$ & $\begin{array}{r}1805 . \\
8 \\
\end{array}$ & 31.8 & 1863.7 & 29.1 & $\begin{array}{r}1928 . \\
9 \\
\end{array}$ & 49.1 & 1928.9 & 49.1 & 93.6 \\
\hline NBH8-11 & 877 & $\begin{array}{r}18069 \\
5\end{array}$ & 9.3 & $\begin{array}{r}8.459 \\
7 \\
\end{array}$ & 3.6 & 4.6749 & 5.8 & $\begin{array}{r}0.286 \\
8 \\
\end{array}$ & $\begin{array}{r}4 . \\
5\end{array}$ & $\begin{array}{r}0.7 \\
8 \\
\end{array}$ & $\begin{array}{r}1625 . \\
7\end{array}$ & 64.7 & 1762.8 & 48.5 & $\begin{array}{r}1929 . \\
3\end{array}$ & 65.2 & 1929.3 & 65.2 & 84.3 \\
\hline NBH8-79 & 283 & $\begin{array}{r}18258 \\
0 \\
\end{array}$ & 4.2 & $\begin{array}{r}8.441 \\
5 \\
\end{array}$ & 4.7 & 5.5508 & 4.9 & $\begin{array}{r}0.339 \\
8 \\
\end{array}$ & $\begin{array}{r}1 . \\
3 \\
\end{array}$ & $\begin{array}{r}0.2 \\
7 \\
\end{array}$ & $\begin{array}{r}1885 . \\
9 \\
\end{array}$ & 21.7 & 1908.5 & 42.0 & $\begin{array}{r}1933 . \\
2 \\
\end{array}$ & 84.2 & 1933.2 & 84.2 & 97.6 \\
\hline NBH8-45 & 761 & $\begin{array}{r}29267 \\
5 \\
\end{array}$ & 4.9 & $\begin{array}{r}8.386 \\
5 \\
\end{array}$ & 3.2 & 5.3164 & 3.3 & $\begin{array}{r}0.323 \\
4 \\
\end{array}$ & $\begin{array}{r}1 . \\
0\end{array}$ & $\begin{array}{r}0.3 \\
0 \\
\end{array}$ & $\begin{array}{r}1806 . \\
2 \\
\end{array}$ & 15.8 & 1871.5 & 28.3 & $\begin{array}{r}1944 . \\
8 \\
\end{array}$ & 56.3 & 1944.8 & 56.3 & 92.9 \\
\hline NBH8-10 & $\begin{array}{r}128 \\
8 \\
\end{array}$ & $\begin{array}{r}31279 \\
0 \\
\end{array}$ & 48.8 & $\begin{array}{r}8.360 \\
0 \\
\end{array}$ & 4.0 & 5.4710 & 4.1 & $\begin{array}{r}0.331 \\
7 \\
\end{array}$ & $\begin{array}{r}1 . \\
0 \\
\end{array}$ & $\begin{array}{r}0.2 \\
4 \\
\end{array}$ & $\begin{array}{r}1846 . \\
7 \\
\end{array}$ & 16.1 & 1896.1 & 35.4 & $\begin{array}{r}1950 . \\
5 \\
\end{array}$ & 71.5 & 1950.5 & 71.5 & 94.7 \\
\hline $\mathrm{NBH} 8-12$ & $\begin{array}{r}124 \\
8 \\
\end{array}$ & $\begin{array}{r}31618 \\
5 \\
\end{array}$ & 3.2 & $\begin{array}{r}8.336 \\
5 \\
\end{array}$ & 2.7 & 5.6204 & 3.0 & $\begin{array}{r}0.339 \\
8 \\
\end{array}$ & $\begin{array}{r}1 . \\
3 \\
\end{array}$ & $\begin{array}{r}0.4 \\
4 \\
\end{array}$ & $\begin{array}{r}1885 . \\
8 \\
\end{array}$ & 21.9 & 1919.2 & 26.1 & $\begin{array}{r}1955 . \\
5 \\
\end{array}$ & 48.6 & 1955.5 & 48.6 & 96.4 \\
\hline NBH8-81 & 247 & 90115 & 1.2 & $\begin{array}{r}8.327 \\
2\end{array}$ & 2.0 & 5.8676 & 2.5 & $\begin{array}{r}0.354 \\
4\end{array}$ & $\begin{array}{r}1 . \\
5\end{array}$ & $\begin{array}{r}0.5 \\
8\end{array}$ & $\begin{array}{r}1955 . \\
4\end{array}$ & 24.5 & 1956.5 & 21.7 & $\begin{array}{r}1957 . \\
5\end{array}$ & 36.4 & 1957.5 & 36.4 & 99.9 \\
\hline NBH8-6 & 90 & 72050 & 2.7 & $\begin{array}{r}8.295 \\
0 \\
\end{array}$ & 3.2 & 5.8499 & 3.3 & $\begin{array}{r}0.351 \\
9 \\
\end{array}$ & $\begin{array}{r}1 . \\
0 \\
\end{array}$ & $\begin{array}{r}0.3 \\
0 \\
\end{array}$ & $\begin{array}{r}1943 . \\
8 \\
\end{array}$ & 16.8 & 1953.8 & 29.0 & $\begin{array}{r}1964 . \\
4 \\
\end{array}$ & 56.9 & 1964.4 & 56.9 & 99.0 \\
\hline NBH8-77 & 446 & 12932 & 1.4 & 8.286 & 3.6 & 5.6343 & 4.2 & 0.338 & 2. & 0.5 & 1880. & 33.9 & 1921.4 & 35.9 & 1966. & 64.2 & 1966.3 & 64.2 & 95.6 \\
\hline
\end{tabular}




\begin{tabular}{|c|c|c|c|c|c|c|c|c|c|c|c|c|c|c|c|c|c|c|c|}
\hline & & 0 & & 2 & & & & 6 & 1 & 0 & 0 & & & & 3 & & & & \\
\hline $\mathrm{NBH} 8-50$ & 523 & $\begin{array}{r}31824 \\
0 \\
\end{array}$ & 2.3 & $\begin{array}{r}8.284 \\
8 \\
\end{array}$ & 3.6 & 5.9330 & 3.7 & $\begin{array}{r}0.356 \\
5 \\
\end{array}$ & $\begin{array}{l}1 . \\
0\end{array}$ & $\begin{array}{r}0.2 \\
7 \\
\end{array}$ & $\begin{array}{r}1965 . \\
5 \\
\end{array}$ & 16.9 & 1966.1 & 32.3 & $\begin{array}{r}1966 . \\
6 \\
\end{array}$ & 63.9 & 1966.6 & 63.9 & 99.9 \\
\hline NBH8-5 & $\begin{array}{r}107 \\
5 \\
\end{array}$ & 43125 & 5.5 & $\begin{array}{r}8.277 \\
4 \\
\end{array}$ & 4.2 & 5.0666 & 5.3 & $\begin{array}{r}0.304 \\
2 \\
\end{array}$ & $\begin{array}{r}3 . \\
2 \\
\end{array}$ & $\begin{array}{r}0.6 \\
0 \\
\end{array}$ & $\begin{array}{r}1711 . \\
9 \\
\end{array}$ & 47.7 & 1830.5 & 44.6 & $\begin{array}{r}1968 . \\
2 \\
\end{array}$ & 74.7 & 1968.2 & 74.7 & 87.0 \\
\hline NBH8-52 & 504 & $\begin{array}{r}13916 \\
5 \\
\end{array}$ & 73.2 & $\begin{array}{r}8.222 \\
7 \\
\end{array}$ & 4.2 & 6.1907 & 4.3 & $\begin{array}{r}0.369 \\
2 \\
\end{array}$ & $\begin{array}{r}1 . \\
0\end{array}$ & $\begin{array}{r}0.2 \\
3 \\
\end{array}$ & $\begin{array}{r}2025 . \\
6 \\
\end{array}$ & 17.4 & 2003.1 & 37.3 & $\begin{array}{r}1980 . \\
0\end{array}$ & 73.9 & 1980.0 & 73.9 & $\begin{array}{r}102 . \\
3 \\
\end{array}$ \\
\hline NBH8-57 & $\begin{array}{r}124 \\
2 \\
\end{array}$ & $\begin{array}{r}33155 \\
5 \\
\end{array}$ & 2.4 & $\begin{array}{r}8.215 \\
9 \\
\end{array}$ & 2.6 & 5.6091 & 2.8 & $\begin{array}{r}0.334 \\
2 \\
\end{array}$ & $\begin{array}{r}1 . \\
0\end{array}$ & $\begin{array}{r}0.3 \\
6 \\
\end{array}$ & $\begin{array}{r}1858 . \\
8 \\
\end{array}$ & 16.1 & 1917.5 & 23.8 & $\begin{array}{r}1981 . \\
5 \\
\end{array}$ & 45.8 & 1981.5 & 45.8 & 93.8 \\
\hline $\mathrm{NBH} 8-54$ & 437 & $\begin{array}{r}25978 \\
0 \\
\end{array}$ & 2.4 & $\begin{array}{r}8.196 \\
8 \\
\end{array}$ & 5.7 & 5.6889 & 6.2 & $\begin{array}{r}0.338 \\
2 \\
\end{array}$ & $\begin{array}{r}2 . \\
4 \\
\end{array}$ & $\begin{array}{r}0.3 \\
8 \\
\end{array}$ & $\begin{array}{r}1878 . \\
0 \\
\end{array}$ & 38.4 & 1929.7 & 53.2 & $\begin{array}{r}1985 . \\
7 \\
\end{array}$ & $\begin{array}{r}101 . \\
2 \\
\end{array}$ & 1985.7 & $\begin{array}{r}101 . \\
2 \\
\end{array}$ & 94.6 \\
\hline NBH8-21 & 330 & $\begin{array}{r}11522 \\
0 \\
\end{array}$ & 4.0 & $\begin{array}{r}8.182 \\
0 \\
\end{array}$ & 5.0 & 5.3583 & 5.3 & $\begin{array}{r}0.318 \\
0 \\
\end{array}$ & $\begin{array}{r}1 . \\
6 \\
\end{array}$ & $\begin{array}{r}0.3 \\
1 \\
\end{array}$ & $\begin{array}{r}1779 . \\
8 \\
\end{array}$ & 25.0 & 1878.2 & 45.0 & $\begin{array}{r}1988 . \\
9 \\
\end{array}$ & 89.0 & 1988.9 & 89.0 & 89.5 \\
\hline NBH8-46 & 453 & $\begin{array}{r}15759 \\
5 \\
\end{array}$ & 3.9 & $\begin{array}{r}8.074 \\
3 \\
\end{array}$ & 2.7 & 5.3524 & 3.6 & $\begin{array}{r}0.313 \\
4 \\
\end{array}$ & $\begin{array}{r}2 . \\
4 \\
\end{array}$ & $\begin{array}{r}0.6 \\
7 \\
\end{array}$ & $\begin{array}{r}1757 . \\
6 \\
\end{array}$ & 36.9 & 1877.3 & 30.9 & $\begin{array}{r}2012 . \\
4 \\
\end{array}$ & 47.7 & 2012.4 & 47.7 & 87.3 \\
\hline $\mathrm{NBH} 8-30$ & 132 & 15795 & 1.7 & $\begin{array}{r}8.057 \\
5 \\
\end{array}$ & 2.9 & 5.7535 & 3.6 & $\begin{array}{r}0.336 \\
2 \\
\end{array}$ & $\begin{array}{r}2 . \\
1 \\
\end{array}$ & $\begin{array}{r}0.5 \\
9 \\
\end{array}$ & $\begin{array}{r}1868 . \\
5 \\
\end{array}$ & 34.2 & 1939.4 & 30.9 & $\begin{array}{r}2016 . \\
1 \\
\end{array}$ & 51.2 & 2016.1 & 51.2 & 92.7 \\
\hline NBH8-41 & 140 & 30000 & 1.7 & $\begin{array}{r}7.946 \\
1 \\
\end{array}$ & 2.8 & 5.4689 & 3.1 & $\begin{array}{r}0.315 \\
2 \\
\end{array}$ & $\begin{array}{r}1 . \\
2 \\
\end{array}$ & $\begin{array}{r}0.3 \\
8 \\
\end{array}$ & $\begin{array}{r}1766 . \\
1 \\
\end{array}$ & 18.2 & 1895.7 & 26.4 & $\begin{array}{r}2040 . \\
8 \\
\end{array}$ & 50.2 & 2040.8 & 50.2 & 86.5 \\
\hline $\mathrm{NBH} 8-33$ & 713 & $\begin{array}{r}18821 \\
0 \\
\end{array}$ & 2.5 & $\begin{array}{r}7.854 \\
0 \\
\end{array}$ & 3.8 & 5.9007 & 4.6 & $\begin{array}{r}0.336 \\
1 \\
\end{array}$ & $\begin{array}{r}2 . \\
5 \\
\end{array}$ & $\begin{array}{r}0.5 \\
5 \\
\end{array}$ & $\begin{array}{r}1868 . \\
0 \\
\end{array}$ & 40.7 & 1961.3 & 39.8 & $\begin{array}{r}2061 . \\
3 \\
\end{array}$ & 67.6 & 2061.3 & 67.6 & 90.6 \\
\hline $\mathrm{NBH} 8-35$ & 409 & $\begin{array}{r}16823 \\
0 \\
\end{array}$ & 0.7 & $\begin{array}{r}7.580 \\
0 \\
\end{array}$ & 2.0 & 7.1504 & 2.2 & $\begin{array}{r}0.393 \\
1 \\
\end{array}$ & $\begin{array}{r}1 . \\
0\end{array}$ & $\begin{array}{r}0.4 \\
5 \\
\end{array}$ & $\begin{array}{r}2137 . \\
2 \\
\end{array}$ & 18.2 & 2130.3 & 20.0 & $\begin{array}{r}2123 . \\
7 \\
\end{array}$ & 35.2 & 2123.7 & 35.2 & $\begin{array}{r}100 . \\
6 \\
\end{array}$ \\
\hline NBH8-53 & 275 & $\begin{array}{r}22314 \\
5 \\
\end{array}$ & 2.1 & $\begin{array}{r}7.577 \\
2 \\
\end{array}$ & 2.7 & 6.5369 & 3.7 & $\begin{array}{r}0.359 \\
2 \\
\end{array}$ & $\begin{array}{r}2 . \\
5 \\
\end{array}$ & $\begin{array}{r}0.6 \\
7 \\
\end{array}$ & $\begin{array}{r}1978 . \\
5 \\
\end{array}$ & 42.1 & 2050.9 & 32.5 & $\begin{array}{r}2124 . \\
4 \\
\end{array}$ & 48.0 & 2124.4 & 48.0 & 93.1 \\
\hline $\mathrm{NBH} 8-23$ & $\begin{array}{r}116 \\
8 \\
\end{array}$ & $\begin{array}{r}18016 \\
0 \\
\end{array}$ & 2.8 & $\begin{array}{r}7.348 \\
8 \\
\end{array}$ & 4.7 & 6.0770 & 5.8 & $\begin{array}{r}0.323 \\
9 \\
\end{array}$ & $\begin{array}{r}3 . \\
3 \\
\end{array}$ & $\begin{array}{r}0.5 \\
8 \\
\end{array}$ & $\begin{array}{r}1808 . \\
7 \\
\end{array}$ & 52.7 & 1987.0 & 50.6 & $\begin{array}{r}2177 . \\
8 \\
\end{array}$ & 82.6 & 2177.8 & 82.6 & 83.1 \\
\hline $\mathrm{NBH} 8-27$ & 132 & 82700 & 1.3 & $\begin{array}{r}7.279 \\
4 \\
\end{array}$ & 2.5 & 7.7872 & 2.7 & $\begin{array}{r}0.411 \\
1 \\
\end{array}$ & $\begin{array}{l}1 . \\
0\end{array}$ & $\begin{array}{r}0.3 \\
7 \\
\end{array}$ & $\begin{array}{r}2220 . \\
1 \\
\end{array}$ & 18.8 & 2206.7 & 24.5 & $\begin{array}{r}2194 . \\
3 \\
\end{array}$ & 44.0 & 2194.3 & 44.0 & $\begin{array}{r}101 . \\
2 \\
\end{array}$ \\
\hline NBH8-76 & 372 & 32590 & 2.7 & $\begin{array}{r}7.191 \\
9 \\
\end{array}$ & 5.8 & 8.2588 & 6.5 & $\begin{array}{r}0.430 \\
8 \\
\end{array}$ & $\begin{array}{r}3 . \\
1 \\
\end{array}$ & $\begin{array}{r}0.4 \\
7 \\
\end{array}$ & $\begin{array}{r}2309 . \\
2 \\
\end{array}$ & 59.4 & 2259.8 & 59.3 & $\begin{array}{r}2215 . \\
3 \\
\end{array}$ & $\begin{array}{r}100 . \\
3 \\
\end{array}$ & 2215.3 & $\begin{array}{r}100 . \\
3 \\
\end{array}$ & $\begin{array}{r}104 . \\
2 \\
\end{array}$ \\
\hline $\mathrm{NBH} 8-22$ & 143 & 51865 & 1.2 & $\begin{array}{r}7.067 \\
2 \\
\end{array}$ & 3.8 & 8.2894 & 5.0 & $\begin{array}{r}0.424 \\
9 \\
\end{array}$ & $\begin{array}{r}3 . \\
3 \\
\end{array}$ & $\begin{array}{r}0.6 \\
6 \\
\end{array}$ & $\begin{array}{r}2282 . \\
6 \\
\end{array}$ & 63.1 & 2263.2 & 45.2 & $\begin{array}{r}2245 . \\
6 \\
\end{array}$ & 65.0 & 2245.6 & 65.0 & $\begin{array}{r}101 . \\
6 \\
\end{array}$ \\
\hline NBH8-40 & 110 & 57315 & 1.2 & $\begin{array}{r}7.044 \\
2\end{array}$ & 5.6 & 7.9226 & 7.8 & $\begin{array}{r}0.404 \\
8\end{array}$ & $\begin{array}{r}5 . \\
4\end{array}$ & $\begin{array}{r}0.6 \\
9\end{array}$ & $\begin{array}{r}2190 . \\
9\end{array}$ & 99.6 & 2222.2 & 70.1 & $\begin{array}{r}2251 . \\
2\end{array}$ & 97.0 & 2251.2 & 97.0 & 97.3 \\
\hline NBH8-3 & 108 & 61560 & 2.1 & $\begin{array}{r}6.757 \\
2 \\
\end{array}$ & 4.5 & 8.9129 & 5.5 & $\begin{array}{r}0.436 \\
8 \\
\end{array}$ & $\begin{array}{r}3 . \\
2 \\
\end{array}$ & $\begin{array}{r}0.5 \\
8 \\
\end{array}$ & $\begin{array}{r}2336 . \\
3 \\
\end{array}$ & 62.5 & 2329.1 & 50.3 & $\begin{array}{r}2322 . \\
8 \\
\end{array}$ & 77.0 & 2322.8 & 77.0 & $\begin{array}{r}100 . \\
6 \\
\end{array}$ \\
\hline NBH8-62 & 66 & 75755 & 1.4 & $\begin{array}{r}6.624 \\
5 \\
\end{array}$ & 3.3 & 8.7293 & 3.4 & $\begin{array}{r}0.419 \\
4 \\
\end{array}$ & $\begin{array}{c}1 . \\
1 \\
\end{array}$ & $\begin{array}{r}0.3 \\
2 \\
\end{array}$ & $\begin{array}{r}2257 . \\
8 \\
\end{array}$ & 20.8 & 2310.1 & 31.4 & $\begin{array}{r}2356 . \\
8 \\
\end{array}$ & 55.9 & 2356.8 & 55.9 & 95.8 \\
\hline NBH8-13 & 308 & $\begin{array}{r}19353 \\
0 \\
\end{array}$ & 1.1 & $\begin{array}{r}6.158 \\
7 \\
\end{array}$ & 3.5 & $\begin{array}{r}10.070 \\
0 \\
\end{array}$ & 3.8 & $\begin{array}{r}0.449 \\
8 \\
\end{array}$ & $\begin{array}{r}1 . \\
3 \\
\end{array}$ & $\begin{array}{r}0.3 \\
5 \\
\end{array}$ & $\begin{array}{r}2394 . \\
4 \\
\end{array}$ & 26.8 & 2441.2 & 35.0 & $\begin{array}{r}2480 . \\
5 \\
\end{array}$ & 59.7 & 2480.5 & 59.7 & 96.5 \\
\hline $\mathrm{NBH} 8-47$ & 307 & $\begin{array}{r}38370 \\
0 \\
\end{array}$ & 1.3 & $\begin{array}{r}5.975 \\
9 \\
\end{array}$ & 4.3 & 9.9040 & 4.5 & $\begin{array}{r}0.429 \\
3\end{array}$ & $\begin{array}{r}1 . \\
6\end{array}$ & $\begin{array}{r}0.3 \\
5 \\
\end{array}$ & $\begin{array}{r}2302 . \\
4\end{array}$ & 30.4 & 2425.9 & 42.0 & $\begin{array}{r}2531 . \\
2\end{array}$ & 71.7 & 2531.2 & 71.7 & 91.0 \\
\hline NBH8-1 & 306 & $\begin{array}{r}20670 \\
0\end{array}$ & 2.1 & $\begin{array}{r}5.955 \\
7\end{array}$ & 5.4 & $\begin{array}{r}10.885 \\
4\end{array}$ & 5.5 & $\begin{array}{r}0.470 \\
2\end{array}$ & $\begin{array}{r}1 . \\
2\end{array}$ & $\begin{array}{r}0.2 \\
1\end{array}$ & $\begin{array}{r}2484 . \\
4\end{array}$ & 24.5 & 2513.4 & 51.6 & $\begin{array}{r}2536 . \\
9\end{array}$ & 90.8 & 2536.9 & 90.8 & 97.9 \\
\hline $\mathrm{NBH} 8-20$ & 469 & $\begin{array}{r}17941 \\
0 \\
\end{array}$ & 1.4 & $\begin{array}{r}5.953 \\
1 \\
\end{array}$ & 3.0 & $\begin{array}{r}10.737 \\
4 \\
\end{array}$ & 3.6 & $\begin{array}{r}0.463 \\
6 \\
\end{array}$ & $\begin{array}{r}2 . \\
1 \\
\end{array}$ & $\begin{array}{r}0.5 \\
7 \\
\end{array}$ & $\begin{array}{r}2455 . \\
4 \\
\end{array}$ & 42.1 & 2500.7 & 33.8 & $\begin{array}{r}2537 . \\
6 \\
\end{array}$ & 50.3 & 2537.6 & 50.3 & 96.8 \\
\hline
\end{tabular}


1. All uncertainties are reported at the 1-sigma level, and include only measurement errors. Systematic errors would increase age uncertainties by $1-2 \%$

2. $U$ concentration and $U / T h$ are calibrated relative to our Sri Lanka standard zircon, and are accurate to

$\sim 20 \%$.

3. Common $\mathrm{Pb}$ correction is from 204Pb, with composition interpreted from Stacey and Kramers (1975) and uncertainties of $1.0 \mathrm{for} 206 \mathrm{~Pb} / 204 \mathrm{~Pb}, 0.3$ for $207 \mathrm{~Pb} /$

$204 \mathrm{~Pb}$,

and 2.0 for $208 \mathrm{~Pb} / 204 \mathrm{~Pb}$

4. $\mathrm{U} / \mathrm{Pb}$ and $206 \mathrm{~Pb} / 207 \mathrm{~Pb}$ fractionation is calibrated relative to fragments of a large Sri Lanka zircon of $563 \pm 3.2 \mathrm{Ma}$ (2-sigma) (Gehrels et al., 2008).

5. $\mathrm{U}$ decay constants and composition as follows: $238 \mathrm{U}=9.8485 \times 10-10,235 \mathrm{U}=1.55125 \times 10-10,238 \mathrm{U} / 235 \mathrm{U}=137.88$

6. Concordance generally not shown (NA) for analyses with best ages $<500 \mathrm{Ma}$, because $206 \mathrm{~Pb} / 207 \mathrm{~Pb}$ ages are not reliable for analyses these young analyses.

7. All samples were ablated with 35 micron-diameter beam, except for BU07-54, BU07-55, and BU08-72, which had smaller zircons and were hit with a 25 micron-diameter beam, and

therefore have lower precision, particularly for ${ }^{206} \mathrm{~Pb} /{ }^{207} \mathrm{~Pb}$ 
Table DR2:

\begin{tabular}{|c|c|c|c|c|c|}
\hline & $\begin{array}{c}206 \mathrm{~Pb} / 238 \mathrm{U} \\
\text { s.e. (\%) }\end{array}$ & $\begin{array}{c}206 \mathrm{~Pb} / 207 \mathrm{~Pb} \\
\text { s.e. }(\%)\end{array}$ & $\begin{array}{c}206 \mathrm{~Pb} / 238 \mathrm{U} \\
\text { standard } \\
\text { ave. age } \\
\text { (Ma) }\end{array}$ & $\begin{array}{c}206 \mathrm{~Pb} / 238 \mathrm{U} \\
\text { standard } \\
\text { std. dev. } \\
\text { (Ma) }\end{array}$ & $\begin{array}{c}\text { age } \\
\text { cutoff } \\
\text { (Ma) }\end{array}$ \\
\hline BU07-53 & 2.4 & 1.1 & 565 & 15 & 970 \\
\hline BU07-54 & 1.4 & 1.2 & 564 & 13 & 970 \\
\hline BU08-135 & 2.1 & 1 & 564 & 2 & 970 \\
\hline BU08-72 & & & 564 & 3 & 970 \\
\hline BU08-18 & 3.3 & 1.2 & 563 & 3 & 1100 \\
\hline $\mathrm{NBH}-3$ & 3.3 & 1.1 & 565 & 15 & 1000 \\
\hline $\mathrm{NBH}-4$ & 4.2 & 1.4 & 562 & 24 & 970 \\
\hline BU07-55 & 2.5 & 1 & 564 & 5 & 970 \\
\hline BU07-43A & 3.4 & 1.3 & 564 & 18 & 970 \\
\hline BU07-42 & 3.5 & 1.2 & 566 & 18 & 970 \\
\hline BU07-22 & 2 & 0.9 & 564 & 11 & 970 \\
\hline BU07-10 & 2.1 & 1.2 & 563 & 12 & 1000 \\
\hline $\mathrm{NBH}-8$ & 2.6 & 1.6 & 564 & 17 & 970 \\
\hline
\end{tabular}


Table DR3: $\delta 13 \mathrm{C}$ and $\delta 18 \mathrm{O}$ values for Baxa Group dolomite (duplicates in bold)

\begin{tabular}{|c|c|c|c|c|c|c|c|c|}
\hline & & $\delta 13 \mathrm{C}$ & & $\delta 180$ & & $\delta 13 \mathrm{C}$ & $\delta 180$ & \\
\hline section ID & sample ID & $(\mathrm{KIS}) \pm$ & (KI & IS ) $\#$ & & (VPDB) & (VPDB) & Max P \\
\hline BHU1 & 2 & 8.18 & 0.04 & -2.26 & 0.07 & 4.22 & -6.86 & 858 \\
\hline BHU1 & 2 & 8.08 & 0.02 & 2.550 & .03 & 4.25 & -7.03 & 1013 \\
\hline BHU1 & 3 & 7.47 & 0.01 & -2.39 & 0.03 & 3.52 & -6.99 & 872 \\
\hline BHU1 & 4 & 7.63 & 0.01 & -2.43 & 0.03 & 3.68 & -7.03 & 1090 \\
\hline BHU1 & 5 & 6.79 & 0.04 & -2.82 & 0.03 & 2.85 & -7.42 & 1058 \\
\hline BHU1 & 6 & 7.42 & 0.05 & -1.19 & 0.11 & 3.47 & -5.80 & 1041 \\
\hline BHU1 & 6 & 7.15 & $0.03-1$ & .360 . & 03 & 3.40 & -5.93 & 909 \\
\hline BHU1 & 8 & 7.29 & 0.02 & -2.45 & 0.02 & 3.34 & -7.06 & 1070 \\
\hline BHU1 & 9 & 7.54 & 0.02 & -1.16 & 0.02 & 3.71 & -5.65 & 1193 \\
\hline BHU1 & 10 & 8.66 & 0.02 & -0.12 & 0.02 & 4.70 & -4.75 & 528 \\
\hline BHU1 & 11 & 8.92 & 0.02 & 0.14 & 0.03 & 4.96 & -4.49 & 1109 \\
\hline BHU1 & 12 & 7.95 & 0.06 & 0.31 & 0.08 & 4.00 & -4.31 & 350 \\
\hline BHU1 & 12 & 7.750 . & $\mathbf{0 3}$ & 0.130. & 01 & 4.00 & -4.43 & 919 \\
\hline BHU1 & 13 & 7.37 & 0.02 & -1.69 & 0.04 & 3.55 & -6.17 & 650 \\
\hline BHU1 & 14 & 8.10 & 0.04 & -1.57 & 0.05 & 4.14 & -6.18 & 955 \\
\hline BHU1 & 15 & 7.66 & 0.03 & 2.15 & 0.06 & 3.71 & -2.49 & 1148 \\
\hline BHU1 & 16 & 7.13 & 0.02 & -2.03 & 0.05 & 3.37 & -6.59 & 292 \\
\hline BHU1 & 17 & 7.26 & 0.03 & -2.78 & 0.02 & 3.43 & -7.26 & 568 \\
\hline BHU1 & 18 & 7.65 & 0.03 & -1.34 & 0.02 & 3.90 & -5.90 & 786 \\
\hline \multirow[t]{2}{*}{ BHU1 } & 18 & 7.96 & 0.05 . & 1.200 & .08 & 4.00 & -5.81 & 1224 \\
\hline & strat level (m) & & & & & & & \\
\hline CBU1 & 1.0 & 8.84 & 0.02 & -1.35 & 0.01 & 5.04 & -5.82 & 1589 \\
\hline CBU1 & 1.0 & 8.87 & 0.01 & 1.470 & .02 & 5.07 & -5.87 & 1303 \\
\hline CBU1 & 2.3 & 10.71 & 0.01 & 0.73 & 0.01 & 6.92 & -3.73 & 1869 \\
\hline CBU1 & 3.6 & 10.45 & 0.01 & -0.77 & 0.02 & 6.66 & -5.24 & 1309 \\
\hline CBU1 & 3.6 & 10.38 & 0.02 & 0.610 & .03 & 6.57 & -5.30 & 1253 \\
\hline CBU1 & 5.1 & 9.92 & 0.01 & -5.24 & 0.02 & 6.13 & -9.66 & 795 \\
\hline CBU1 & 5.1 & 9.91 & 0.01 & 5.150 & .02 & 6.12 & -9.65 & 1519 \\
\hline CBU1 & 5.8 & 9.96 & 0.01 & -0.73 & 0.02 & 6.17 & -5.13 & 1163 \\
\hline CBU1 & 6.8 & 9.54 & 0.02 & 0.57 & 0.03 & 5.75 & -3.89 & 1819 \\
\hline CBU1 & 7.9 & 9.75 & 0.01 & 0.85 & 0.02 & 5.96 & -3.54 & 1183 \\
\hline CBU1 & 13.9 & 9.31 & 0.02 & -1.55 & 0.05 & 5.52 & -6.02 & 1779 \\
\hline CBU1 & 13.9 & 9.33 & $0.02-1$ & .640. & 02 & 5.53 & -6.04 & 689 \\
\hline CBU1 & 15.5 & 8.79 & 0.01 & -2.19 & 0.02 & 5.00 & -6.60 & 923 \\
\hline CBU1 & 21.0 & 8.62 & 0.03 & -5.07 & 0.03 & 4.83 & -9.57 & 1679 \\
\hline CBU1 & 26.6 & 8.69 & 0.03 & -4.92 & 0.05 & 4.89 & -9.42 & 1689 \\
\hline CBU1 & 26.6 & 8.73 & $0.01-5$ & .040 & 03 & 4.93 & -9.46 & 779 \\
\hline
\end{tabular}




\begin{tabular}{|c|c|c|c|c|c|c|c|c|}
\hline CBU1 & 31.6 & 10.12 & 0.01 & 1.23 & 0.02 & 6.33 & -3.16 & 823 \\
\hline CBU1 & 32.6 & 9.82 & 0.02 & -3.32 & 0.04 & 6.03 & -7.81 & 799 \\
\hline CBU1 & 33.6 & 9.99 & 0.01 & -3.37 & 0.03 & 6.20 & -7.79 & 648 \\
\hline CBU1 & 34.6 & 9.28 & 0.02 & -4.33 & 0.04 & 5.49 & -8.82 & 1649 \\
\hline CBU1 & 34.6 & 9.33 & $\mathbf{0 . 0 1}-4$ & $4 \quad .450$. & 02 & 5.54 & -8.87 & 753 \\
\hline CBU1 & 37.4 & 9.24 & 0.02 & -2.68 & 0.03 & 5.45 & -7.17 & 1649 \\
\hline CBU1 & 40.3 & 9.82 & 0.01 & -1.99 & 0.03 & 6.03 & -6.47 & 1619 \\
\hline CBU1 & 41.2 & 9.69 & 0.00 & -2.31 & 0.02 & 5.90 & -6.72 & 1243 \\
\hline CBU1 & 43.1 & 9.18 & 0.01 & -4.82 & 0.02 & 5.39 & -9.32 & 1289 \\
\hline CBU1 & 43.1 & 9.18 & $\mathbf{0 . 0 2}-4$ & $\begin{array}{r}870 \\
\end{array}$ & 02 & 5.38 & -9.30 & 738 \\
\hline CBU1 & 47.2 & 9.15 & 0.01 & -3.47 & 0.01 & 5.36 & -7.96 & 1549 \\
\hline CBU1 & 48.3 & 9.10 & 0.04 & -4.25 & 0.07 & 5.31 & -8.68 & 1233 \\
\hline CBU1 & 55.6 & 7.19 & 0.01 & -0.35 & 0.02 & 3.39 & -4.82 & 1079 \\
\hline CBU1 & 58.6 & 5.35 & 0.01 & -2.45 & 0.01 & 1.53 & -6.86 & 1123 \\
\hline CBU1 & 58.6 & 5.33 & $\mathbf{0 . 0 3}-2$ & 2.400. & $\mathbf{0 3}$ & 1.51 & -6.81 & 745 \\
\hline CBU1 & 60.1 & 4.97 & 0.01 & -1.87 & 0.01 & 1.16 & -6.35 & 1549 \\
\hline CBU1 & 63.3 & 4.54 & 0.01 & -2.75 & 0.02 & 0.71 & -7.17 & 863 \\
\hline CBU1 & 67.0 & 6.34 & 0.00 & -2.44 & 0.01 & 2.53 & -6.93 & 1219 \\
\hline CBU1 & 67.0 & 6.28 & 0.01 & 2.280 & .03 & 2.50 & -6.96 & 1302 \\
\hline CBU1 & 68.0 & 5.12 & 0.02 & -2.87 & 0.02 & 1.30 & -7.28 & 1083 \\
\hline CBU1 & 68.0 & 5.06 & 0.01 & 2.620 & .03 & 1.28 & -7.30 & 1283 \\
\hline CBU1 & 73.5 & 6.21 & 0.01 & -3.26 & 0.03 & 2.40 & -7.75 & 1769 \\
\hline CBU1 & 75.0 & 5.17 & 0.02 & -4.32 & 0.02 & 1.35 & -8.75 & 735 \\
\hline CBU1 & 76.5 & 5.68 & 0.01 & -2.90 & 0.03 & 1.88 & -7.38 & 1609 \\
\hline CBU1 & 76.5 & 5.67 & 0.01 & 3.010 & .01 & 1.85 & -7.42 & 1013 \\
\hline CBU2 & 0.0 & 9.47 & 0.02 & 0.74 & 0.02 & 5.67 & -3.95 & 1263 \\
\hline CBU2 & 1.0 & 8.93 & 0.01 & -1.18 & 0.02 & 5.14 & -5.87 & 1263 \\
\hline CBU2 & 5.0 & 8.43 & 0.03 & 0.38 & 0.03 & 4.64 & -4.31 & 1295 \\
\hline CBU2 & 5.0 & 8.460 & .01 & 0.510 & .01 & 4.67 & -4.18 & 1148 \\
\hline CBU2 & 8.0 & 8.82 & 0.01 & -0.40 & 0.03 & 5.02 & -5.09 & 1231 \\
\hline CBU2 & 10.0 & 8.97 & 0.01 & 0.03 & 0.03 & 5.17 & -4.67 & 1310 \\
\hline CBU2 & 12.0 & 8.22 & 0.02 & -1.07 & 0.03 & 4.43 & -5.75 & 1207 \\
\hline CBU2 & 14.0 & 8.72 & 0.02 & 0.02 & 0.02 & 4.92 & -4.67 & 1153 \\
\hline CBU2 & 14.8 & 9.08 & 0.03 & 1.20 & 0.01 & 5.29 & -3.49 & 1278 \\
\hline CBU2 & 16.0 & 9.48 & 0.03 & 0.76 & 0.03 & 5.68 & -3.93 & 1219 \\
\hline CBU2 & 18.0 & 8.83 & 0.02 & -2.71 & 0.03 & 5.03 & -7.39 & 1205 \\
\hline CBU2 & 20.0 & 8.20 & 0.02 & -5.06 & 0.02 & 4.41 & -9.73 & 1136 \\
\hline CBU2 & 31.0 & 8.59 & 0.02 & -1.07 & 0.05 & 4.80 & -5.75 & 1192 \\
\hline CBU2 & 33.0 & 8.75 & 0.03 & -0.48 & 0.04 & 4.95 & -5.17 & 1234 \\
\hline CBU2 & 35.0 & 8.61 & 0.01 & -3.20 & 0.02 & 4.81 & -7.87 & 1300 \\
\hline
\end{tabular}




\begin{tabular}{|c|c|c|c|c|c|c|c|c|}
\hline CBU2 & 37.0 & 8.59 & 0.01 & -3.81 & 0.03 & 4.79 & -8.48 & 1285 \\
\hline CBU2 & 39.0 & 9.23 & 0.02 & -3.40 & 0.02 & 5.43 & -8.07 & 1183 \\
\hline CBU2 & 41.0 & 8.59 & 0.01 & -3.98 & 0.02 & 4.80 & -8.65 & 1129 \\
\hline CBU2 & 41.0 & 8.57 & 0.02 & 4.090 & .02 & 4.77 & -8.76 & 1256 \\
\hline CBU2 & 48.0 & 8.53 & 0.02 & -1.14 & 0.04 & 4.73 & -5.83 & 1268 \\
\hline CBU2 & 50.0 & 8.65 & 0.02 & -2.74 & 0.02 & 4.85 & -7.42 & 1278 \\
\hline CBU2 & 52.0 & 8.93 & 0.01 & -2.55 & 0.02 & 5.14 & -7.23 & 1180 \\
\hline CBU2 & 53.5 & 8.80 & 0.01 & 0.73 & 0.04 & 5.00 & -3.97 & 1205 \\
\hline CBU2 & 57.0 & 9.00 & 0.02 & -2.43 & 0.03 & 5.20 & -7.11 & 1246 \\
\hline CBU2 & 61.0 & 8.43 & 0.02 & -5.18 & 0.02 & 4.63 & -9.84 & 985 \\
\hline CBU2 & 63.0 & 8.96 & 0.01 & -3.34 & 0.03 & 5.17 & -8.02 & 1097 \\
\hline CBU2 & 65.0 & 8.74 & 0.02 & -2.54 & 0.03 & 4.94 & -7.22 & 1231 \\
\hline CBU2 & 65.2 & 8.02 & 0.02 & -4.63 & 0.03 & 4.23 & -9.30 & 1222 \\
\hline CBU2 & 75.0 & 9.23 & 0.02 & -3.32 & 0.05 & 5.43 & -8.00 & 1053 \\
\hline CBU2 & 85.0 & 5.33 & 0.02 & -5.31 & 0.03 & 1.55 & -9.97 & 1297 \\
\hline CBU2 & 85.0 & 5.39 & 0.04 & -5.39 & 0.03 & 1.59 & -9.89 & 1049 \\
\hline CBU5 & 1.0 & 7.96 & 0.02 & -1.67 & 0.03 & 4.17 & -6.35 & 1129 \\
\hline CBU5 & 3.0 & 10.43 & 0.02 & -2.09 & 0.01 & 6.62 & -6.78 & 1060 \\
\hline CBU5 & 4.0 & 10.28 & 0.01 & 0.68 & 0.03 & 6.48 & -4.01 & 1124 \\
\hline CBU5 & 5.0 & 9.15 & 0.02 & -1.39 & 0.01 & 5.35 & -6.08 & 1248 \\
\hline CBU5 & 5.0 & 9.07 & 0.02 & 1.480 & .03 & 5.27 & -6.16 & 1195 \\
\hline CBU5 & 5.2 & 9.68 & 0.01 & -2.99 & 0.03 & 5.88 & -7.66 & 1156 \\
\hline CBU5 & 6.0 & 7.95 & 0.01 & -2.53 & 0.02 & 4.16 & -7.21 & 1244 \\
\hline CBU5 & 6.1 & 7.33 & 0.02 & -3.66 & 0.02 & 3.54 & -8.34 & 1075 \\
\hline CBU5 & 7.0 & 7.25 & 0.01 & 0.99 & 0.02 & 3.47 & -3.71 & 1273 \\
\hline CBU5 & 8.0 & 7.86 & 0.02 & 0.07 & 0.02 & 4.07 & -4.62 & 1241 \\
\hline CBU5 & 8.0 & 7.890 & .02 & 0.140 & .02 & 4.10 & -4.56 & 1273 \\
\hline CBU5 & 9.0 & 8.13 & 0.04 & 0.78 & 0.03 & 4.34 & -3.92 & 1361 \\
\hline CBU5 & $9 \mathrm{a}$ & 7.07 & 0.01 & -0.37 & 0.03 & 3.28 & -5.06 & 1231 \\
\hline CBU5 & 10.0 & 7.45 & 0.03 & -2.14 & 0.05 & 3.66 & -6.82 & 1229 \\
\hline CBU5 & 11.0 & 8.15 & 0.02 & -0.66 & 0.04 & 4.36 & -5.34 & 1248 \\
\hline CBU5 & 12.0 & 6.54 & 0.02 & -2.87 & 0.03 & 2.76 & -7.55 & 1148 \\
\hline CBU5 & 13.0 & 5.60 & 0.03 & -0.90 & 0.04 & 1.82 & -5.58 & 1224 \\
\hline CBU5 & 14.0 & 7.77 & 0.02 & 0.69 & 0.02 & 3.97 & -3.77 & 1639 \\
\hline CBU5 & 14.0 & 7.800. & 01 & 0.610. & 02 & 4.00 & -3.78 & 427 \\
\hline CBU5 & 15.0 & 7.19 & 0.01 & 1.26 & 0.02 & 3.39 & -3.19 & 1219 \\
\hline CBU5 & 16.0 & 7.76 & 0.01 & -0.54 & 0.02 & 3.96 & -4.94 & 1013 \\
\hline CBU5 & 17.0 & 8.05 & 0.01 & -1.43 & 0.01 & 4.25 & -5.90 & 1319 \\
\hline CBU5 & 17.0 & 8.07 & $0.02-1$ & 1.420. & 02 & 4.27 & -5.83 & 601 \\
\hline CBU5 & 18.0 & 9.26 & 0.02 & -3.52 & 0.02 & 5.47 & -8.01 & 1519 \\
\hline
\end{tabular}




\begin{tabular}{|c|c|c|c|c|c|c|c|c|}
\hline CBU5 & 19.0 & 9.55 & 0.03 & -2.05 & 0.02 & 5.76 & -6.46 & 658 \\
\hline CBU5 & 20.0 & 9.91 & 0.01 & -2.76 & 0.03 & 6.12 & -7.25 & 899 \\
\hline CBU5 & 20.0 & 9.89 & $0.02-2$ & .930 . & 03 & 6.10 & -7.34 & 613 \\
\hline CBU13 & 11.0 & 7.36 & 0.01 & -4.17 & 0.02 & 3.56 & -8.67 & 1719 \\
\hline CBU13 & 11.0 & 7.40 & $\mathbf{0 . 0 7}-4$ & $\begin{array}{l}400 \\
\end{array}$ & 09 & 3.60 & -8.62 & 777 \\
\hline CBU 13 & 13.0 & 8.14 & 0.02 & -0.61 & 0.01 & 4.34 & -5.01 & 798 \\
\hline CBU13 & 22.0 & 8.25 & 0.05 & 0.88 & 0.08 & 4.45 & -3.58 & 1379 \\
\hline CBU13 & 25.0 & 7.06 & 0.03 & -2.76 & 0.02 & 3.26 & -7.18 & 782 \\
\hline CBU13 & 31.0 & 7.96 & 0.02 & 0.33 & 0.01 & 4.17 & -4.14 & 1689 \\
\hline CBU13 & 38.5 & 8.21 & 0.02 & 0.68 & 0.01 & 4.41 & -3.71 & 789 \\
\hline CBU13 & 40.0 & 8.00 & 0.02 & 0.00 & 0.02 & 4.21 & -4.47 & 1679 \\
\hline CBU13 & 40.0 & 8.000 & .01 & 0.020 & .02 & 4.21 & -4.45 & 1209 \\
\hline CBU13 & 57.0 & 7.90 & 0.02 & 0.18 & 0.04 & 4.10 & -4.22 & 933 \\
\hline CBU13 & 63.0 & 8.19 & 0.02 & 0.08 & 0.02 & 4.39 & -4.38 & 1019 \\
\hline CBU13 & 63.0 & 8.240. & \begin{tabular}{|l|}
01 \\
\end{tabular} & 0.010. & $\mathbf{0 3}$ & 4.44 & -4.39 & 798 \\
\hline CBU13 & 72.0 & 7.82 & 0.01 & 0.47 & 0.02 & 4.02 & -4.00 & 1529 \\
\hline CBU13 & 107.0 & 7.27 & 0.01 & 0.25 & 0.02 & 3.46 & -4.14 & 873 \\
\hline CBU 13 & 111.0 & 7.35 & 0.02 & 0.49 & 0.04 & 3.56 & -3.98 & 733 \\
\hline CBU 13 & 111.0 & 7.320. & \begin{tabular}{|l|}
01 \\
\end{tabular} & 0.350 & $\mathbf{0 3}$ & 3.51 & -4.05 & 739 \\
\hline CBU 13 & 127.0 & 6.95 & 0.02 & -0.92 & 0.03 & 3.15 & -5.40 & 1699 \\
\hline CBU 13 & 137.0 & 7.23 & 0.01 & 0.11 & 0.02 & 3.42 & -4.29 & 753 \\
\hline CBU 13 & 141.0 & 7.23 & 0.02 & 0.69 & 0.02 & 3.43 & -3.77 & 686 \\
\hline CBU 13 & 146.0 & 7.37 & 0.01 & -0.11 & 0.01 & 3.57 & -4.51 & 767 \\
\hline CBU 13 & 148.0 & 7.28 & 0.01 & 0.72 & 0.02 & 3.48 & -3.74 & 1099 \\
\hline CBU 13 & 148.0 & 7.290. & 02 & 0.640. & 01 & 3.48 & -3.75 & 642 \\
\hline CBU 13 & 151.0 & 7.31 & 0.01 & 0.05 & 0.02 & 3.51 & -4.41 & 1449 \\
\hline CBU 13 & 156.0 & 7.93 & 0.02 & -1.79 & 0.01 & 4.12 & -6.20 & 432 \\
\hline CBU 13 & 159.0 & 7.31 & 0.02 & 0.35 & 0.03 & 3.51 & -4.12 & 703 \\
\hline CBU 13 & 159.0 & 7.250. & $\mathbf{0 2}$ & 0.230. & $\mathbf{0 3}$ & 3.44 & -4.17 & 665 \\
\hline CBU 13 & 167.0 & 6.81 & 0.03 & -1.66 & 0.04 & 3.01 & -6.14 & 1359 \\
\hline CBU 13 & 177.0 & 7.10 & 0.00 & -2.28 & 0.02 & 3.29 & -6.69 & 743 \\
\hline CBU 13 & 180.0 & 7.63 & 0.02 & -1.27 & 0.03 & 3.84 & -5.75 & 1439 \\
\hline CBU 13 & 180.0 & 7.66 & $0.01-1$ & .310. & 03 & 3.86 & -5.72 & 963 \\
\hline CBU 13 & 182.0 & 7.91 & 0.02 & -0.96 & 0.01 & 4.11 & -5.44 & 775 \\
\hline
\end{tabular}

